\title{
Die Finten im Boxen. \\ Eine Untersuchung am Beispiel der Olympischen Spiele Sydney 2000
}

\begin{abstract}
Dissertation
zur Erlangung des sozialwissenschaftlichen Doktorgrades der Sozialwissenschaftlichen Fakultät der Universität Göttingen
\end{abstract}

vorgelegt

von

AYMAN RASHAD HAFEZ HUSSEIN

aus

EL SHARKIA / ÄGYPTEN

Göttingen

2004 
1. Gutachter: Prof. Dr. ARND KRÜGER

2. Gutachter: PD Dr. MICHAEL BASTIAN

Tag der mündlichen Prüfung: 08.07.2004 


\section{Danksagung}

An erster Stelle möchte ich Herrn Professor Dr. ARND KRÜGER für sein Vertrauen, seine Unterstützung und seinen Rat bei der Erstellung dieser Arbeit meinen Dank aussprechen.

Weiterhin möchte ich Herrn PD Dr. MICHAEL BASTIAN und Herrn Prof. Dr. HANS-DIETER HALLER danken, die als Zweitgutachter bzw. mündlicher Prüfer dieser Arbeit stets aufgeschlossen gegenüberstanden.

Diese Arbeit wäre allerdings ohne die Mitwirkung vieler Helfer nie entstanden. Ich danke dem Boxtrainer GEGHAM HAKOBIAN aus Armenien, der als Experte eine Analyse von fünf Boxkämpfen vorgenommen hat, um die notwendige Objektivität der Analyse zu gewährleisten. Im Besonderen die Korrekturhinweise und die Übersetzungshilfen durch Herrn MATTHIAS KERSCHNITZKE zu erwähnen.

Besonderen Dank gebührt auch dem Deutschen Amateurboxverband Kassel für die Überlassung der Unterlagen über die Auslosung der Boxkämpfe bei den Olympischen Spielen in Sydney 2000.

Schließlich möchte ich meinen speziellen Dank meiner Familie, insbesondere meiner Mutter und meiner Frau, für die Geduld und das Verständnis und die moralische Unterstützung während meiner Studien in Deutschland ausdrücken. Ich danke auch der Ägyptischen Regierung für die finanzielle Unterstützung für meinen Studienaufenthalt in Deutschland.

AYMAN HUSSEIN, Göttingen 2004 


\section{WIDMUNG}

Diese Arbeit widme ich meiner Familie, insbesondere dem Gedenken an meinen Vater 


\section{INHALTSVERZEICHNIS}

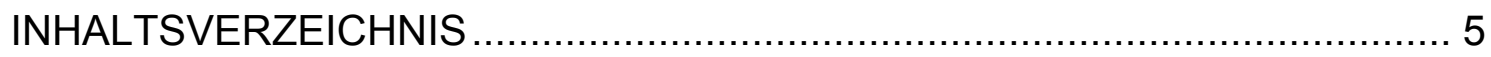

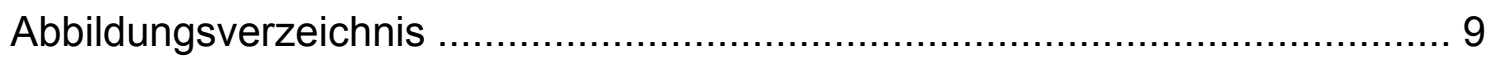

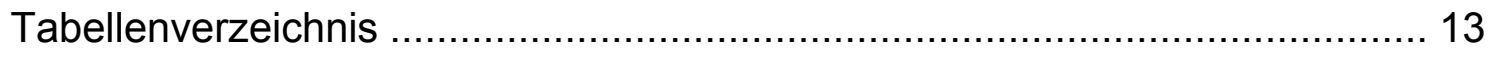

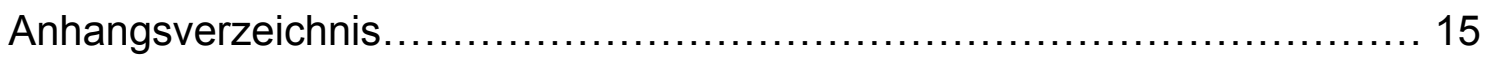

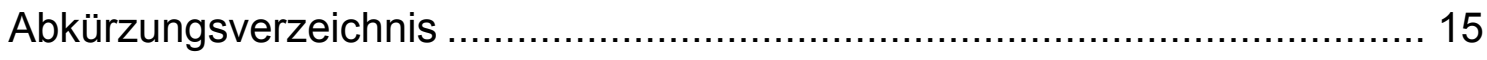

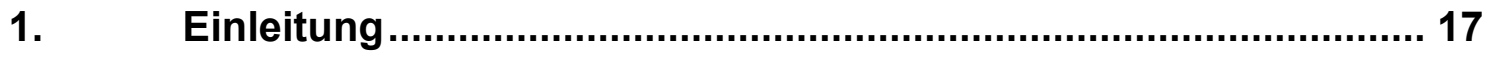

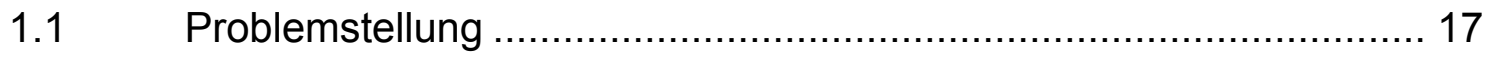

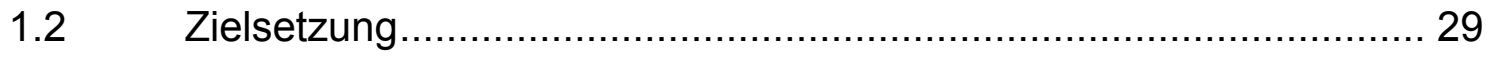

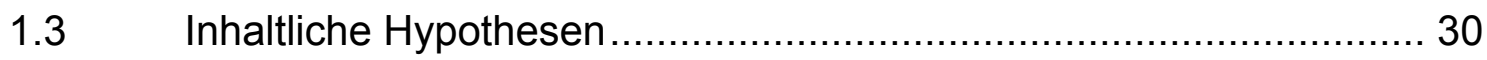

1.4 Definitionen von Täuschungshandlungen im Sport ..................... 31

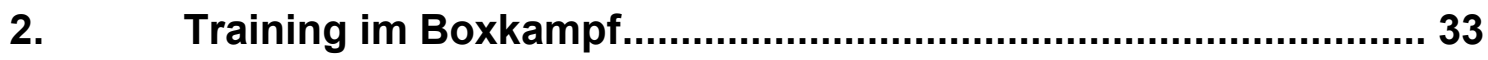

$2.1 \quad$ Techniktraining im Boxen .................................................... 33

Elementares Schnelligkeitstraining im Boxen ........................... 35

2.3 Komplexes Schnelligkeitstraining im Boxen........................... 36

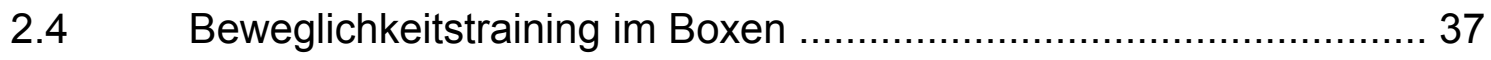

Koordinationstraining im Boxen ....................................... 39

2.6 Koordination und Techniktraining im Boxen............................. 40

2.7 Training der kognitiven Fertigkeiten im Boxen .......................... 42

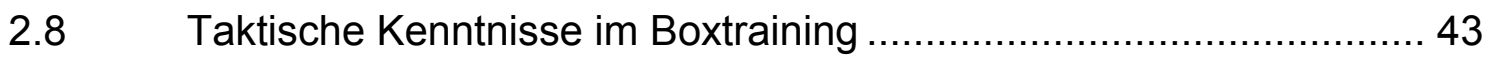

2.9 Das Training der Taktik im Boxen .................................... 44

2.10 Das Training taktischer Fähigkeiten im Boxen .......................... 45

2.11 Das Training taktischer Fertigkeiten im Boxen.......................... 46 
2.12 Trainingspraxis im Boxen....................................................... 46

3. Technik und Taktik im Boxen..................................................... 48

3.1 Forschung über die Technik und Taktik im Boxen......................... 48

3.2 Die technischen Grundelemente des Boxens............................... 50

3.3 Die Boxstellung (Kampfstellung) ………….............................. 50

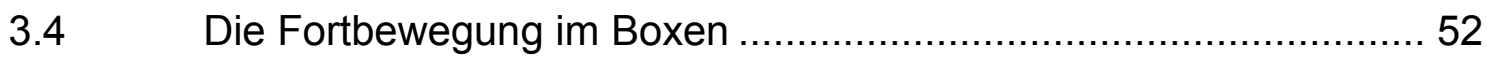

3.5 Die Grundschläge im Boxen .................................................... 53

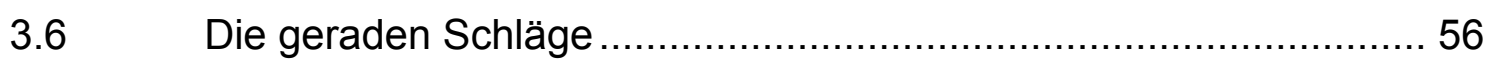

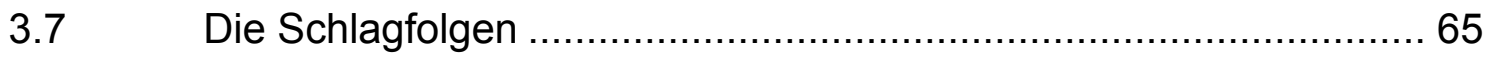

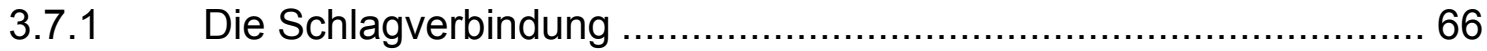

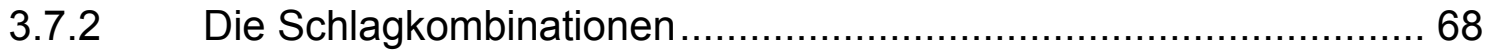

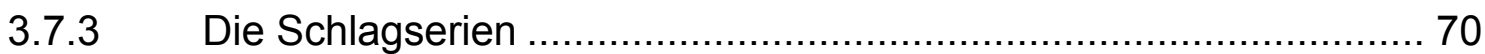

3.8 Die Verteidigung im Boxen .................................................... 71

3.8.1 Die Verteidigungswichtigkeit des Boxen..................................... 71

3.8.2 Die erfolgreichen Verteidigungsgrundsätze ................................ 71

3.8.3 Die Verteidigungsarten ....................................................... 72

3.8.4 Die Verteidigungsmethode …................................................... 74

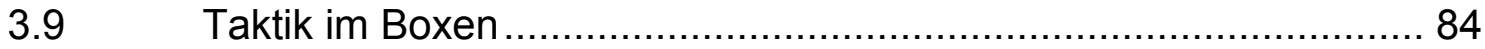

3.10 Die taktische Führung des Kampfes.......................................... 89

3.11 Das Verhalten im Boxring ...................................................... 91

4. Die Täuschung im Boxsport................................................... 102

4.1 Theoretische Betrachtung von Täuschungshandlungen im Boxsport

4.2 Handlungsregulation bei Täuschungshandlungen im Boxsport.... 104

4.3 Orientierungs- und Entscheidungsregulation bei Täuschungen im

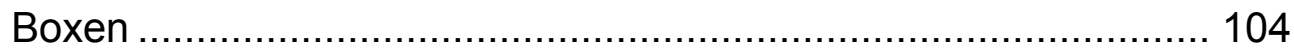

4.4 Maßnahmen zum Management begrenzter kognitiver

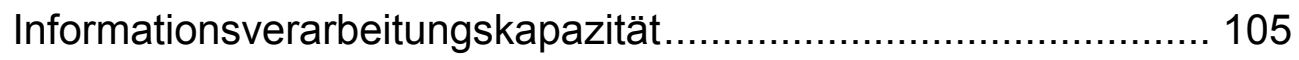

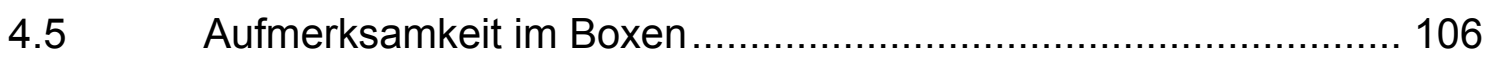

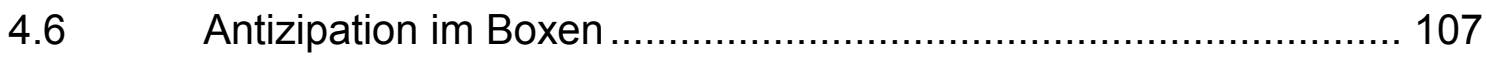

4.7 Wahrnehmung in Täuschungssituationen im Boxen .................... 109

4.8 Ausführungs- und Kontroll- Regulation im Boxkampf ................... 111 
4.8.1 Auswirkung gelungener Finten und Täuschungsaufstellung .

4.8.2 Bewegungsausführung bei Finten und Imitationstäuschungen im Boxen

5. Die Finten im Boxen.

5.1 Der Angriff unter der Verwendung von Finten .......................... 115

5.2 Die Angriffschläge ..................................................... 116

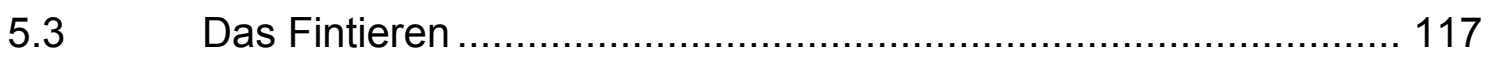

Arten der Finten im Boxen ............................................. 119

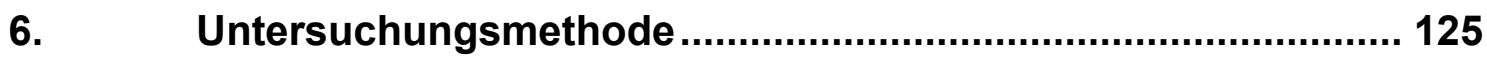

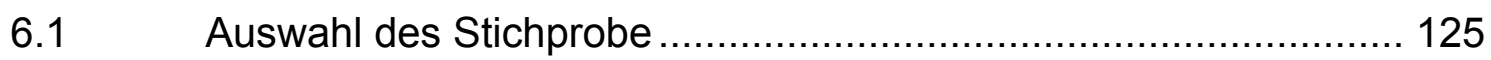

6.2 Konzeption und Auswertung der Kämpfe ............................. 126

Reliabilität....................................................................... 126

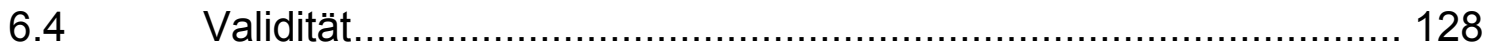

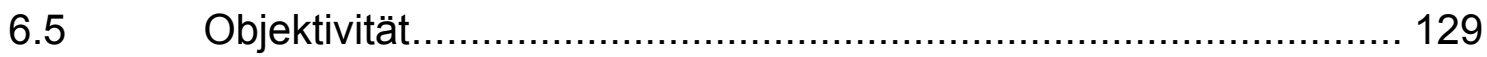

7. Statistische Auswertung ..................................................... 131

8. Ergebnisse und Schlussfolgerung ...................................... 133

8.1 Der Sieger verwendet mehr Finten....................................... 133

8.2 Der Sieger verwendet mehr kombinierte Finten ........................ 135

8.3 Der Sieger verwendet mehr variable kombinierte Finten............. 137

8.4 Schwere Boxer verwenden Finten häufiger als leichte Boxer........ 139

8.5 Schwere Boxer verwenden kombinierte Finten häufiger als leichte

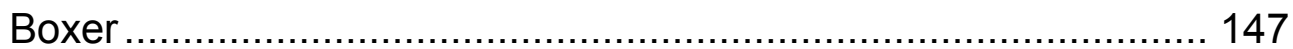

8.6 Der Sieger macht gegen Ende des Kampfes häufiger variable Finten als der Verlierer.

8.6.1 Schwere Sieger machen gegen Ende des Kampfes häufiger variable Finten als leichte Sieger . 157

8.6.2 Schwere Boxer machen gegen Ende des Kampfes häufiger variable Finten als leichte Boxer..... 159

8.7 Die Sieger variieren die Art der Finten von Runde zu Runde stärker als die Verlierer 
8.8 Nationale Boxschulen

8.8.1 Die amerikanische Boxschule unterscheidet sich von denen aus Russland, der Ukraine, Kuba und Kasachstan 169

8.8.2 Die russische Boxschule unterscheidet sich von denen aus der Ukraine, Kuba und Kasachstan

8.8.3 Die kubanische Boxschule unterscheidet sich von denen aus der Ukraine und Kasachstan 184

8.8.4 Die kasachische Boxschule unterscheidet sich von der aus Ukraine 188

9. Diskussion der Ergebnisse 190

10. Schlussfolgerungen und Nachbetrachtungen 197

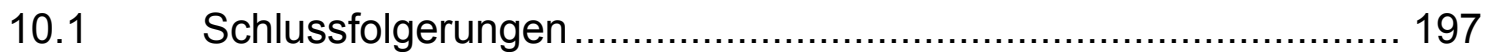

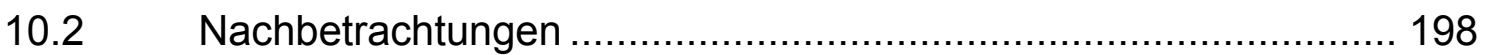

11. Zusammenfassung ............................................................. 199

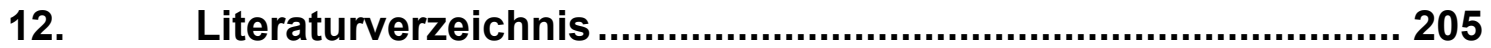

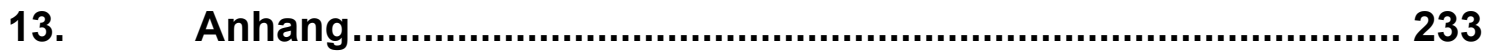




\section{Abbildungsverzeichnis}

Abb. 1: Die Hände der Kämpfer waren durch lange Lederriemen geschützt (336 v. Chr.).

Abb. 2: Boxer mit Minihanteln geformten Handschuhen. Römischer

Marmorsarcophagus (500 v. Chr.).

Abb. 3: Boxer, eine geöffnete Hand verwendend, um seinen Konkurrenten zu

blockieren, Griechische Vase (550 v. Chr.) ............................. 19

Abb. 4: Das Bandagieren der Hände .................................................. 22

Abb. 5: Systematisierung der Boxtaktik........................................... 45

Abb. 6: Die Boxauslagen in verschiedenen Distanzen ............................. 51

Abb. 7: Fortbewegung schreitend- gleitend nach vorn, hinten, rechts und links53

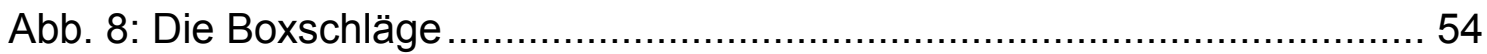

Abb. 9: Die Schlagempfindlichen Körperstellen (K. - O.-Punkte) des Boxers.. 55

Abb. 10: Die linke Gerade zum Kopf aus dem Stand von vorn und von der Seite

Abb. 11: Die linke Gerade zum Kopf mit einem Schritt nach vorn (A), nach hinten (B), nach links (C) und nach rechts (D) ......................... 59

Abb. 12: Die linke Gerade zum Oberkörper aus dem Stand .......................60 60

Abb. 13: Die Gerade mit der Führungshand zum Oberkörper in Bewegung .... 61

Abb. 14: Die Gerade mit der Schlaghand zum Kopf im Stand .......................62 62

Abb. 15: Die Gerade mit der Schlaghand zum Kopf in Bewegung ..................63 63

Abb. 16: Die Gerade mit der Schlaghand zum Oberkörper aus dem Stand ..... 64

Abb. 17: Die Gerade mit der Schlaghand zum Oberkörper in Bewegung ........ 64

Abb. 18: Zweierschlagverbindung gerader Stoß zum Kopf ..........................66

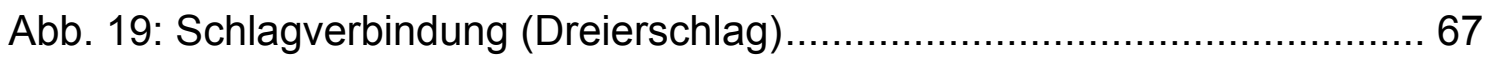

Abb. 20: Schlagkombination: der erste von zwei Schlägen und der zweite von

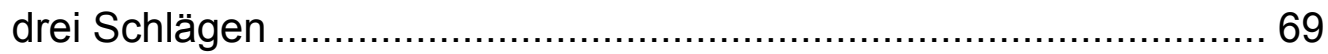

Abb. 21: Eine Schlagserie aus sechs Schlägen ................................... 71

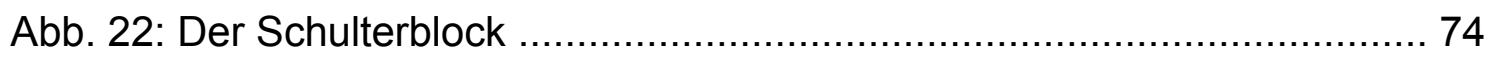

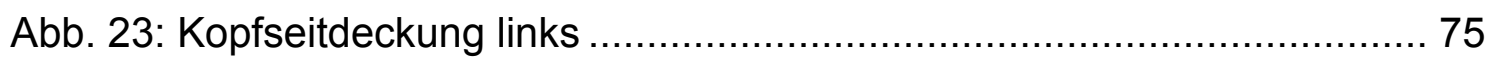

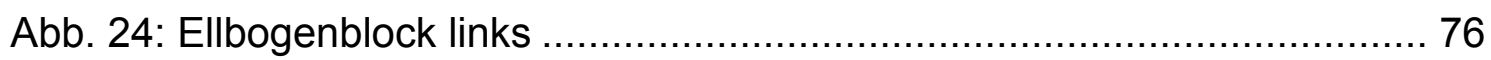

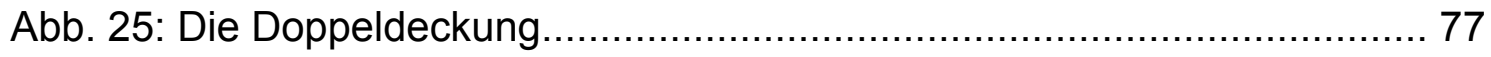

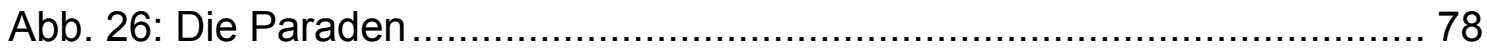


Abb. 27: Abducken nach rechts und nach links mit einer rechter Geraden zum Oberkörper

Abb. 28: Abducken nach unten 80

Abb. 39: Vermeiden des Seitwärtshakens mit der Schlaghand zum Kopf durch

Abrollen nach rechts. 81

Abb. 30: Pendeln des Boxers im Schlagbereich 81

Abb. 31: Ausweichen durch Rückschritt 83

Abb. 32: Die Beinbewegungen beim Sidestep nach rechts $(A)$ und links $(B) \ldots 83$

Abb. 33: Die Rolle der Finten bei den taktischen Haupthandlungen im Boxen 87

Abb. 34: Zeitlicher Ablauf von Schlag und Reaktion

Abb. 35: Finten mit halbem Vorwärtsschritt und halbem Rückwärtsschritt..... 120

Abb. 36: Klassifizierung der einfachen Finten

Abb. 37: Klassifizierung der kombinierten Finten

Abb. 38: Positionierung der einzelnen Kameras aus der Sicht der

Deckenkamera

Abb. 39: Der Sieger ( $n=28)$ verwendet mehr Finten als der Verlierer $(n=28) .134$

Abb. 40: Der Sieger $(n=28)$ verwendet mehr Kombinierte Finten als der

Verlierer $(n=28)$

Abb. 41: Der Sieger $(n=28)$ verwendet mehr variable Kombinierte Finten als der Verlierer $(n=28)$

Abb. 42: Boxer der sechs schwersten Gewichtsklassen (Weltergewicht, Halbmittelgewicht, Mittelgewicht, Halbschwergewicht, Schwergewicht und Superschwergewicht) $(n=28)$ verwenden Finten häufiger als Boxer der sechs leichtesten Gewichtsklassen (Halbfliegengewicht, Fliegengewicht, Bantamgewicht, Federgewicht, Leichtgewicht und Halbweltergewicht) $(\mathrm{n}=28)$ 140

Abb. 43: Boxer der fünf schwersten Gewichtsklassen (Halbmittelgewicht, Mittelgewicht, Halbschwergewicht, Schwergewicht und Superschwergewicht) ( $n=26$ ) verwenden Finten häufiger als Boxer der fünf leichtesten Gewichtsklassen (Halbfliegengewicht, Fliegengewicht, Bantamgewicht, Federgewicht und Leichtgewicht) $(n=26)$.

Abb. 44: Boxer der vier schwersten Gewichtsklassen (Mittelgewicht, Halbschwergewicht, Schwergewicht und Superschwergewicht) ( $n=22)$ verwenden Finten häufiger als Boxer der vier leichtesten 
Gewichtsklassen (Halbfliegengewicht, Fliegengewicht, Bantamgewicht und Federgewicht) ( $n=22)$

Abb. 45: Boxer der drei schwersten Gewichtsklassen (Halbschwergewicht, Schwergewicht und Superschwergewicht) $(n=18)$ verwenden Finten häufiger als Boxer der drei leichtesten Gewichtsklassen (Halbfliegengewicht, Fliegengewicht und Bantamgewicht) (n=18) ... 146

Abb. 46: Boxer der sechs schwersten Gewichtsklassen (Weltergewicht, Halbmittelgewicht, Mittelgewicht, Halbschwergewicht, Schwergewicht und Superschwergewicht) $(n=28)$ verwenden kombinierte Finten häufiger als Boxer der sechs leichtesten Gewichtsklassen (Halbfliegengewicht, Fliegengewicht, Bantamgewicht, Federgewicht, Leichtgewicht und Halbweltergewicht) $(n=28)$. 148

Abb. 47: Boxer der fünf schwersten Gewichtsklassen (Halbmittelgewicht, Mittelgewicht, Halbschwergewicht, Schwergewicht und Superschwergewicht) ( $n=26$ ) verwenden kombinierte Finten häufiger als Boxer der fünf leichtesten Gewichtsklassen (Halbfliegengewicht, Fliegengewicht, Bantamgewicht, Federgewicht und Leichtgewicht) $(n=26)$.

Abb. 48: Boxer der vier schwersten Gewichtsklassen (Mittelgewicht, Halbschwergewicht, Schwergewicht und Superschwergewicht) $(n=22)$ verwenden kombinierte Finten häufiger als Boxer der vier leichtesten Gewichtsklassen (Halbfliegengewicht, Fliegengewicht, Bantamgewicht und Federgewicht) $(\mathrm{n}=22)$. 152

Abb. 49: Boxer der drei schwersten Gewichtsklassen (Halbschwergewicht, Schwergewicht und Superschwergewicht) $(n=18)$ verwenden kombinierte Finten häufiger als Boxer der vier leichtesten Gewichtsklassen (Halbfliegengewicht, Fliegengewicht und Bantamgewicht) $(\mathrm{n}=18)$ 154

Abb. 50: Unterschiede zwischen den Siegern $(n=28)$ und den Verlierern $(n=28)$ bei variablen Finten in Abhängigkeit zur Kampfdauer 156

Abb. 51: Unterschiede zwischen den schweren Siegern $(n=14)$ und den leichten Siegern $(n=14)$ bei variablen Finten in Abhängigkeit zur Kampfdauer 
Abb. 52: Unterschiede zwischen den schweren Siegern $(n=28)$ und den leichten Siegern $(n=28)$ bei variablen Finten in Abhängigkeit zur Kampfdauer

Abb. 53: In der ersten Runde variiert der Sieger $(n=28)$ die Art der Finten stärker als die Verlierer $(n=28)$.

Abb. 54: In der zweiten Runde variiert der Sieger $(n=28)$ die Art der Finten stärker als der Verlierer $(n=28)$

Abb. 55: In der dritten Runde variiert der Sieger $(n=28)$ die Art der Finten stärker als der Verlierer $(\mathrm{n}=28)$

Abb. 56: In der vierten Runde variiert der Sieger $(n=28)$ die Art der Finten stärker als die Verlierer $(n=28)$

Abb. 57: Die amerikanischen $(n=4)$ Fintierstile unterscheiden sich von den russischen ( $n=11)$ Fintierstilen.

Abb. 58: Die amerikanischen $(n=4)$ Fintierstile unterscheiden sich von den ukrainischen $(n=5)$ Fintierstilen.

Abb. 59: Die amerikanischen $(n=4)$ Fintierstile unterscheiden sich von den kubanischen $(n=8)$ Fintierstilen.

Abb. 60: Die amerikanischen $(n=4)$ Fintierstile unterscheiden sich von den kasachischen $(n=6)$ Fintierstilen.

Abb. 61: Die russischen $(n=11)$ Fintierstile unterscheiden sich von den ukrainischen $(n=5)$ Fintierstilen.

Abb. 62: Die russischen $(n=11)$ Fintierstile unterscheiden sich von den kubanischen $(n=8)$ Fintierstilen.

Abb. 63: Die russischen $(n=11)$ Fintierstile unterscheiden sich von den kasachischen $(n=6)$ Fintierstilen.

Abb. 64: Die kubanischen $(n=8)$ Fintierstile unterscheiden sich von den ukrainischen $(n=5)$ Fintierstilen.

Abb. 65: Die kubanischen $(n=8)$ Fintierstile unterscheiden sich von den kasachischen $(n=6)$ Fintierstilen.

Abb. 66: Die kasachischen $(n=6)$ Fintierstile unterscheiden sich von den ukrainischen $(n=5)$ Fintierstilen. 


\section{Tabellenverzeichnis}

Tab 1: Berechnung des Rangkorrelationskoeffizienten 130

Tab 2: Anzahl der von Siegern $(n=28)$ bzw. Verlierern $(n=28)$ verwendeten

Finten

Tab 3: Anzahl der von Siegern $(n=28)$ bzw. Verlierern $(n=28)$ verwendeten

Kombinierten Finten

Tab 4: Der Sieger $(n=28)$ verwendet mehr variable Kombinierte Finten als der

Verlierer $(n=28)$

Tab 5: Boxer der sechs schwersten Gewichtsklassen $(n=28)$ verwenden Finten

häufiger als Boxer der sechs leichtesten Gewichtsklassen ( $n=28) \ldots 139$

Tab 6: Boxer der fünf schwersten Gewichtsklassen $(n=26)$ verwenden Finten

häufiger als Boxer der fünf leichtesten Gewichtsklassen $(n=26) \ldots . . .141$

Tab 7: Boxer der vier schwersten Gewichtsklassen $(n=22)$ verwenden Finten

häufiger als Boxer der vier leichtesten Gewichtsklassen ( $n=22)$...... 143

Tab 8: Boxer der drei schwersten Gewichtsklassen $(n=18)$ verwenden Finten

häufiger als Boxer der drei leichtesten Gewichtsklassen $(n=18) \ldots . . .145$

Tab 9: Boxer der sechs schwersten Gewichtsklassen $(n=28)$ verwenden

Kombinierte Finten häufiger als Boxer der sechs leichtesten

Gewichtsklassen ( $n=28)$

Tab 10: Boxer der fünf schwersten Gewichtsklassen $(n=26)$ verwenden

Kombinierte Finten häufiger als Boxer der fünf leichtesten

Gewichtsklassen $(n=26)$.

Tab 11: Boxer der vier schwersten Gewichtsklassen $(n=22)$ verwenden

kombinierte Finten häufiger als Boxer der vier leichtesten

Gewichtsklassen ( $\mathrm{n}=22)$

Tab 12: Boxer der drei schwersten Gewichtsklassen $(n=18)$ verwenden kombinierte Finten häufiger als Boxer der drei leichtesten

Gewichtsklassen ( $n=18)$ 153

Tab 13: Der Sieger $(n=28)$ macht gegen Ende des Kampfes häufiger variable

Finten als der Verlierer $(n=28)$. 155

Tab 14: Schwere Sieger $(n=14)$ machen gegen Ende des Kampfes häufiger variable Finten als leichte Sieger $(n=14)$..... 
Tab 15: Schwere Boxer $(n=28)$ machen gegen Ende des Kampfes häufiger variable Finten als leichte Boxer $(n=28)$

Tab 16: In der ersten Runde variiert der Sieger $(n=28)$ die Art der Finten stärker als die Verlierer $(n=28)$.

Tab 17: In der zweiten Runde variiert der Sieger $(n=28)$ die Art der Finten stärker als der Verlierer $(n=28)$.

Tab 18: In der dritten Runde variiert der Sieger $(n=28)$ die Art der Finten stärker als der Verlierer $(n=28)$

Tab 19: In der vierten Runde variiert der Sieger $(n=28)$ die Art der Finten stärker als die Verlierer $(n=28)$

Tab 20: Die amerikanischen $(n=4)$ Fintierstile unterscheiden sich von den russischen $(n=11)$ Fintierstile.

Tab 21: Die amerikanischen $(n=4)$ Fintierstile unterscheiden sich von den ukrainischen $(n=5)$ Fintierstilen.

Tab 22: Die amerikanischen $(n=4)$ Fintierstile unterscheiden sich von den kubanischen $(n=8)$ Fintierstilen.

Tab 23: Die amerikanischen $(n=4)$ Fintierstile unterscheiden sich von den kasachischen $(n=6)$ Fintierstilen.

Tab 24: Die russischen $(n=11)$ Fintierstile unterscheiden sich von den ukrainischen $(n=5)$ Fintierstilen.

Tab 25: Die russischen $(n=11)$ Fintierstile unterscheiden sich von den kubanischen $(n=8)$ Fintierstilen. 180

Tab 26: Die russischen $(n=11)$ Fintierstile unterscheiden sich von den kasachischen $(n=6)$ Fintierstilen.

Tab 27: Die kubanischen $(n=8)$ Fintierstile unterscheiden sich von den ukrainischen $(n=5)$ Fintierstilen.

Tab 28: Die kubanischen $(n=8)$ Fintierstile unterscheiden sich von den kasachischen $(n=6)$ Fintierstilen. 186

Tab 29: Die kasachischen $(n=6)$ Fintierstile unterscheiden sich von den ukrainischen $(n=5)$ Fintierstilen. 


\section{Anhangsverzeichnis}

Anhang 1: E-Mail von Prof. Dr. Arnd Krüger an die Abt. Images and Sound des loc

Anhang 2: Zeitplan Boxen: Sydney 2000.

Anhang 3: Verteilung der Boxer nach Kategorien und Ländern Sydney 2000

Anhang 4: Verteilung der Boxer nach Kategorien und Ländern im Halbfinale. 234

Anhang 5: Verteilung der Boxer nach Kategorien und Ländern im Finale.....234

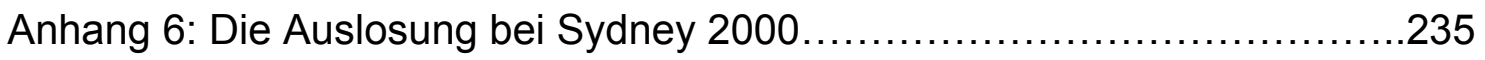

Anhang 7: Formular für die Analysesysteme für jeden Kampf................ 247

Anhang 8: Medaillenspiegel für der Sieger..................................... 248

Anhang 9: Medaillenspiegel für die Länder.............................251

Anhang 10: Gewichts- und Alterklassen Amateure (männlich)............. 252

Anhang 11: Gewichts- und Alterklassen Amateure (weiblich) $\ldots \ldots \ldots \ldots \ldots \ldots . \ldots 253$

Anhang 12: Gewichtsklassen der Profiboxer...............................254

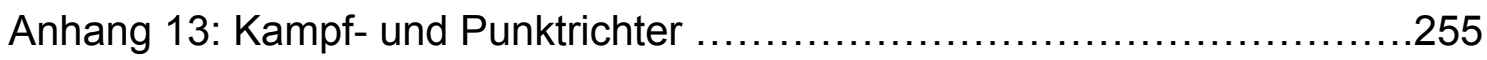


ABKÜRZUNG

IOC

AIBA

HV

HR

Ab

ValR

VaR

VarR

KFi

KFa

KgSA

BSvA

ValBR

VaBR

VarBR

KgSBAV

SSV

BSvBAV

KgSBAZ

SSZ

BSvBAZ

EFB

EFR

EFA

KFBRHSV

KFBAHSV

KFBAHSZ

WBC

WBA

IBF

WBO

\section{BEZEICHNUNG}

$=$ Internationales Olympisches Komitee

$=$ Internationale Amateur-Box-Föderation

= Einfache Finten mit den Beinen (halber Vorwärtsschritt)

$=$ Einfache Finten mit den Beinen (halber Rückwärtsschritt)

$=$ Einfache Finten mit den Beinen (abtauchen)

= Einfache Finten mit dem Rumpf (vorwärts ausweichen links)

= Einfache Finten mit dem Rumpf (vorwärts ausweichen)

= Einfache Finten mit dem Rumpf (vorwärts ausweichen rechts)

= Einfache Finten mit den Armen (Kreisbewegung Fäustling nach innen)

= Einfache Finten mit den Armen (Kreisbewegung Fäustling nach außen)

= Einfache Finten mit den Armen (kurzer gerader Schlag)

$=$ Einfache Finten mit den Armen (Bewegung Schulter nach vorn)

$=$ Kombinierte Finten Beine und Rumpf (halber Schritt nach vorne mit vorwärts ausweichen links)

$=$ Kombinierte Finten Beine und Rumpf (halber Schritt nach vorne mit vorwärts ausweichen)

= Kombinierte Finten Beine und Rumpf (halber Schritt nach vorne mit vorwärts ausweichen rechts)

= Kombinierte Finten Beine und Arme (halber Schritt nach vorne mit kurzem geraden Schlag)

$=$ Kombinierte Finten Beine und Arme (halber Schritt nach vorne mit schwachem Schlag)

$=$ Kombinierte Finten Beine und Arme (halber Schritt nach vorne mit Bewegung der Schulter nach vorn )

$=$ Kombinierte Finten Beine und Arme (halber Schritt zurück mit kurzem geraden Schlag)

= Kombinierte Finten Beine und Arme (halber Schritt zurück mit schwachen Schlag)

$=$ Kombinierte Finten Beine und Arme (halber Schritt zurück

mit Bewegung Schulter nach vorn )

$=$ Einfache Finten mit den Beinen

$=$ Einfache Finten mit dem Rumpf

$=$ Einfache Finten mit den Armen

$=$ Kombinierte Finten Beine und Rumpf (halber Schritt nach vorne)

$=$ Kombinierte Finten Beine und Arme (halber Schritt nach vorne)

= Kombinierte Finten Beine und Arme (halber Schritt zurück)

$=$ World Boxing Council

$=$ World Boxing Association

$=$ International Boxing Federation

$=$ World Boxing Organization 


\section{Einleitung}

\subsection{Problemstellung}

Wenigstens seit dem 7. Jahrtausend v. Chr. übte sich die heranwachsende Jugend und besonders die Männer in verschiedenen Sportarten; bei kultischen Anlässen, Siegesfeiern oder Totenehrungen fanden Wettkämpfe statt. Boxkämpfe unterschiedlicher Art wurden in vielen Gebieten ausgetragen. In China besitzt das Schattenboxen eine sehr alte Tradition, in Indonesien und auf den Südseeinseln kannte man Boxkämpfe, lange bevor Europäer diesen Boden betraten. (vgl. PAPALAS, 1984; WEILER, 1995; BERGER, 1996)

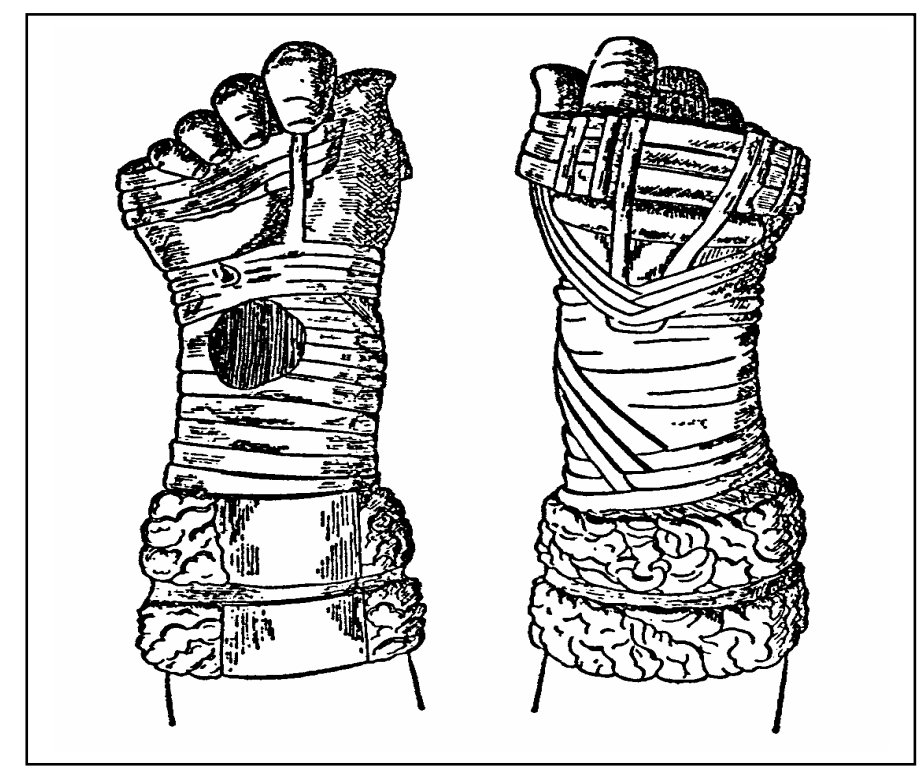

Abb. 1: Die Hände der Kämpfer waren durch lange Lederriemen geschützt (336 v. Chr.) (nach: FINLEY; PLEKET, 1976, 84)

Boxkämpfe wurden in Mittelasien und in Russland ausgetragen. Bis in das 3 . Jahrhundert v. Chr. zeugen Abbildungen in altägyptischen Gräbern und Tempeln von Boxkämpfen; auch den Illyriern und Etruskern war Boxen bekannt. Am meisten ist über den Boxkampf aus der griechisch-römischen Antike überliefert. (vgl. BARISCH, 1953; FIEDLER, 1976; OATES, 1988) 


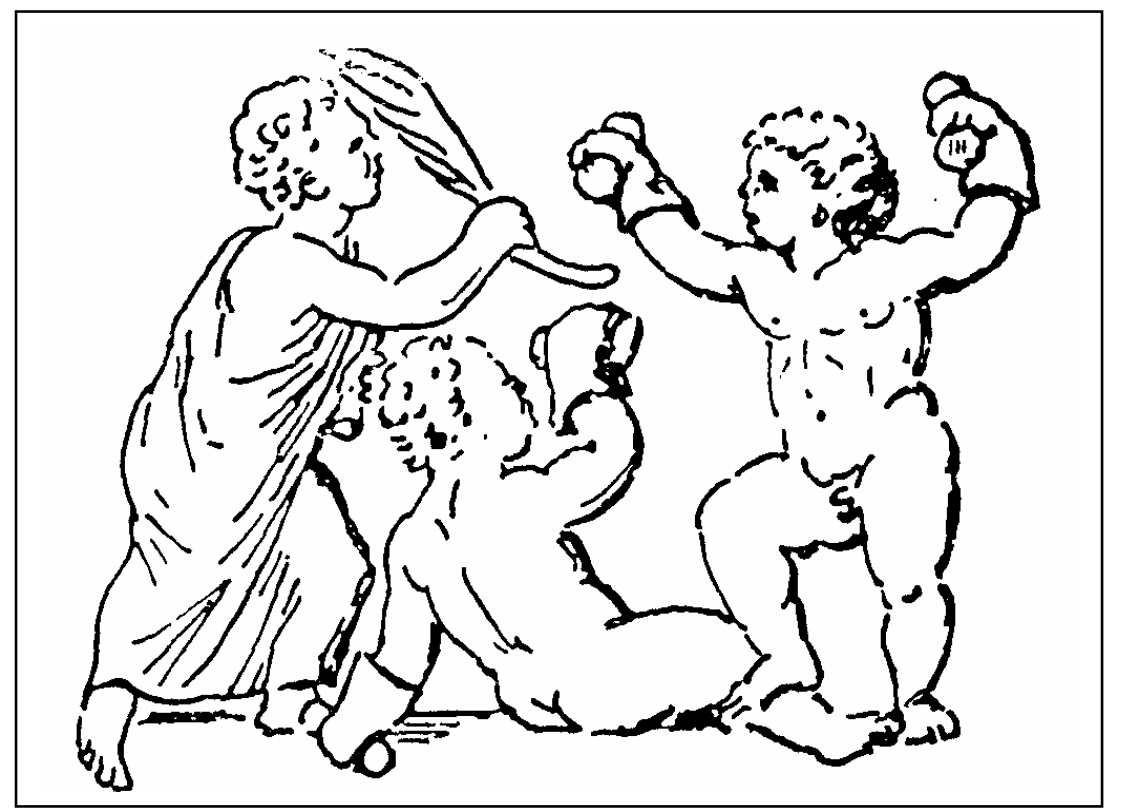

Abb. 2: Boxer mit Minihanteln geformten Handschuhen. Römischer Marmorsarcophagus (500 v. Chr.) (nach: POLIAKOFF, 1987, 76)

Im 8. und im 7. Jahrhundert v. Chr. herrschten im alten Griechenland die Aristokraten, die großen Grundbesitzer. Sie stellten auch die Einzelkämpfer, die Vorkämpfer in den kriegerischen Auseinandersetzungen zwischen den Poleis (Stadtstaaten im alten Griechenland). Sie waren die Wettkämpfer dieser Zeit. Im Krieg und im sportlichen Wettkampf ,,immer der Erste zu sein und den anderen voranzuleuchten", wie Homer es ausdrückt, war das aristokratische Ideal männlicher Tugend. Körperliche Leistungsfähigkeit musste durch Körpererziehung und Sport erworben, erhalten und im Wettkampf bewiesen werden. (vgl. FIEDLER, 1976; FINLEY; PLEKET, 1976; BECKER, 1979; POLIAKOFF, 1989; JÄGER, 1994 )

Die Fertigkeit mit den Fäusten zu kämpfen, ist Jahrtausende alt. Die Entstehung hat viele Facetten, vom förderlichen Kampfsport zur Körperertüchtigung und Wehrfähigkeit bis hin zum gewalttätigen Spektakel. (vgl. HIRSCHFELD, 1953; FIEDLER, 1976; STAUFFER, 1993; WEISE, 1995) 
Etwa siebentausend Jahre alte Darstellungen mit Fäusten kämpfender Männer wurden in den Trümmern eines alten sumerischen Tempels entdeckt. Boxkämpfe lassen sich bis 3000 v. Chr. in Ägypten anhand von Steinrelieffunden nachweisen. Um 900 v. Chr. entwickelte sich der Boxkampf durch die Seevölker zu einer hohen Fertigkeit. (vgl. AMBRUS; BÖHMER u. a., 1985; MCLATCHIE,1986; POLIAKOFF, 1987)

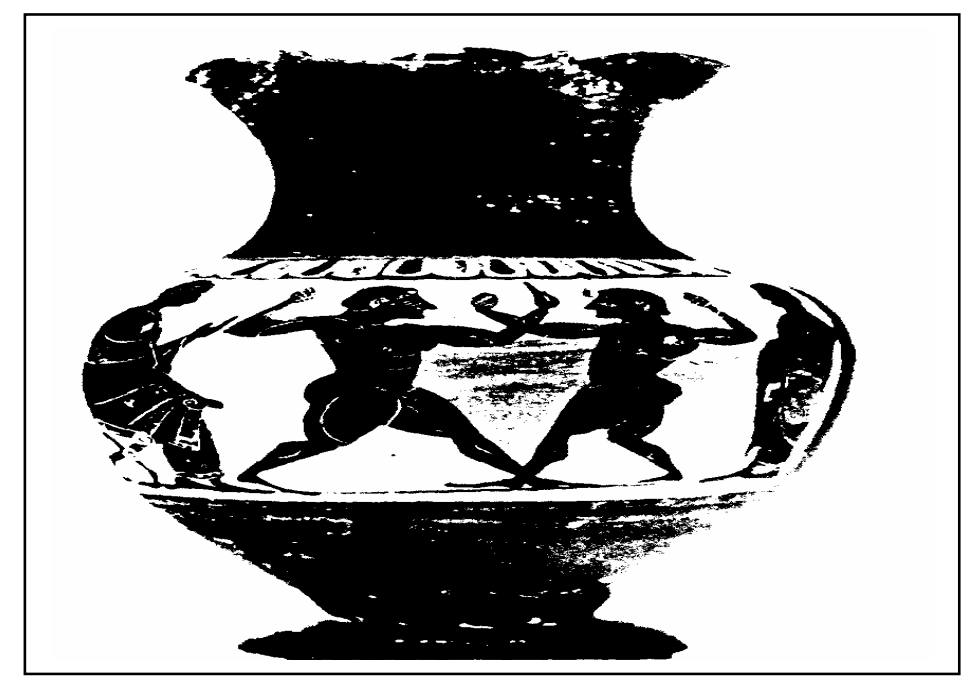

Abb. 3: Boxer, eine geöffnete Hand verwendend, um seinen Konkurrenten zu blockieren, Griechische Vase (550 v. Chr.)

(nach: POLIAKOFF, 1987, 72)

Bei den 23. Olympischen Spielen wurden Boxkämpfe 688 v. Chr. erstmals in Olympia ausgetragen, wobei Onomastos aus Smyrna der erste Sieger war. (vgl. GRADOPOLOW, 1959; FIEDLER, 1976; ELLWANGER; ELLWANGER, 1998)

In der römischen Kaiserzeit wurden die Kämpfe durch die Benutzung der Caesti (Handschuhe, die mit Eisenspitzen oder Bleinägeln durchsetzt waren) immer brutaler, so dass hier Todesfälle durchaus keine Seltenheit mehr waren. Diese grausame Variante des Boxkampfes wurde durch Theodosius schließlich verboten. (vgl. HIRSCHFELD, 1953; BARISCH, 1953; OATES, 1988; SONNENBERG, 1993) 
Diese Vorgänger der heutigen Boxhandschuhe erfuhren zahlreiche Änderungen. Sogar Metallteile wurden auf den Fingergelenken angebracht, um noch brutalere Schläge austeilen zu können. (vgl. BARISCH, 1953; BRAUNE, 1995; DENZ, 1997)

Nachdem das Römische Reich untergegangen war, sind Berichte über diese Sportart eher selten. Im mittelalterlichen Deutschland gab Albrecht Dürer im Auftrage von Kaiser Maximilian ein Fechthandbuch heraus, in welchem auch Boxkampftechniken abgebildet waren. (vgl. FIEDLER, 1976; OATES, 1988; MÜLLER, 1991)

Der moderne Boxkampf entstand nach 1700 in England, wo sich 1719 der Londoner Fechtlehrer James Figg den Titel eines „Champion of England" im Boxkampf zulegte und 1720 die erste Boxschule gründete. In diesen Kämpfen waren die Sitten noch rau. Es war beispielsweise erlaubt, sich gegenseitig die Augen auszuquetschen. Darum entwickelte sich im weiteren Ablauf der Kampf zwar noch ohne Handschuhe, aber bereits nach festen Regeln.

Die Boxhandschuhe wurden 1743 durch Jack Broughton in seinen ,London Prize Rules" vorgeschrieben, um die Verletzungsgefahr zu mindern. (vgl. FIEDLER, 1976; SCHÖFFLER, 1986; OATES, 1988)

In der Form, wie wir es heute kennen, gehörte Boxen bereits zum Wettkampfprogramm der Olympischen Spiele von St. Louis 1904. Die Regelgrundlage waren die „Queensberry Rules", welche 1867 von John Douglas, dem 9. Marquess of Queensberry gemeinsam mit John Graham Chambers aufgestellt wurden und zum Teil mit der Tragepflicht von Boxhandschuhen, dem Bestimmen der Rundendauer auf drei Minuten und dem Zählen bis zehn bei Niederschlag im wesentlichen bis heute (inzwischen mit Ausnahme der Rundendauer bei den Amateuren) Gültigkeit besitzen. (vgl. HIRSCHFELD, 1953; GRADOPOLOW, 1959; FIEDLER, 1976; RÄSCH, 1973; OATES, 1988; WEISE, 1995) 
Obwohl in Deutschland bis 1918 ein polizeiliches Boxverbot bestand, wurde 1912 der erste deutsche Boxverein, der SV Astoria Berlin, von dem Nestor des deutschen Boxsportes, Leonhard Mandler, gegründet. 1920 fand die erste Deutsche Meisterschaft im Amateurboxen statt. (vgl. OATES, 1988; FIEDLER, 1997 )

Nach dem Zweiten Weltkrieg kam es 1946 in London zu einer Neugründung der 1920 in Antwerpen entstandenen FIBA. Der Weltverband der Amateurboxer heißt nun AIBA und ist für wesentliche Regeländerungen verantwortlich. Seit 1950 gibt es den Kampfabbruch beim dritten Niederschlag in einer Runde sowie die Schutzsperre beim Kopf- K.O. und seit 1984 ist das Tragen eines Kopfschutzes im Wettkampf verbindlich. (vgl. OATES, 1988; SONNENBERG, 1989; WEINMANN, 1995; FIEDLER, 1997)

Boxen ist in den neunziger Jahren in Deutschland nochmals zu einem gesellschaftlich akzeptierten, werbewirksamen Massenphänomen geworden. (vgl. FIEDLER, 1976; MEINHARDT, 1996)

Beim Profiboxen sind, anders als beim Amateurboxen, die Sicherheitsbestimmungen noch sehr lückenhaft. 335 Todesfälle im Ring von 1945 bis 1979 sprechen eine deutliche Sprache. 1980 begann man mit einer strikten sportärztliche Überwachung der Faustkämpfer. Dies bedeutete während und nach dem Kampf eine Erweiterung der Entscheidungsbefugnisse des Ringarztes. (vgl. MOORE, 1980; MEINHARDT, 1996)

Generell wird beim Boxen zwischen Amateuren und Berufsboxern unterschieden. Rein äußerlich unterscheiden sich die Amateure von den Profis dadurch, dass sie ein Trikot tragen, während Profiboxer mit nacktem Oberkörper antreten, sowie durch das obligatorische Tragen eines Kopfschutzes bei den Amateuren. Bei den Profiboxern beträgt die Kampfrunde drei Minuten, bei den Amateurboxern beträgt die Kampfrunde seit 1992 zwei Minuten. (vgl. HORNER; LEE u. a., 1993) 
Viel entscheidender sind die Unterschiede in den Handschuhen und Boxbandagen. Im Amateurbereich sind in den niedrigen Klassen bis zum Weltergewicht $(67 \mathrm{~kg})$ Handschuhe mit einem Gewicht von 8 Unzen (228 Gramm), seit 1984 werden ab dem Halbmittelgewicht (ab $67 \mathrm{~kg}$ ) Handschuhe mit 10 Unzen (285 Gramm) Gewicht verwendet. (vgl. OATES, 1988; LEMME, 1991; MAXWELL, 2000)

Die Berufsboxer kämpfen in allen Gewichtsklassen in der Regel mit 6 Unzen Handschuhen. Das höhere Handschuhgewicht in den höheren Klassen der Amateure soll die Schlagwirkung und somit die Schädigungsmöglichkeiten vermindern. Zudem müssen die Amateure im Wettkampf weiche Bandagen (z.B. Mull oder Flanell) mit je 2,50 m Länge und $5 \mathrm{~cm}$ Breite verwenden. Im Training, wenn harte Gerätearbeit auf dem Programm steht, kann man sich auch noch weicher bandagieren. Geben doch die Bandagen der Faust einen festen Halt und vermindern wesentlich die Verletzungsgefahr an Hand-, Daumen- und Fingergelenken. Das Bandagieren der Hände wird individuell meist unterschiedlich gehandhabt. Wichtig aber ist, dass das Handgelenk, die Grundgelenke der Finger und des Daumens sowie die Mittelhandknochen fixiert werden. Die „Profis" benutzen so genannte harte Bandagen (z.B. Gummiband, Pflaster oder Leukoplast, die die Schlagwirkung erhöhen. (vgl. RÄSCH, 1973; FIEDLER, 1976; CHARNAS; PYERITYR, 1986; STAUFFER, 1993)

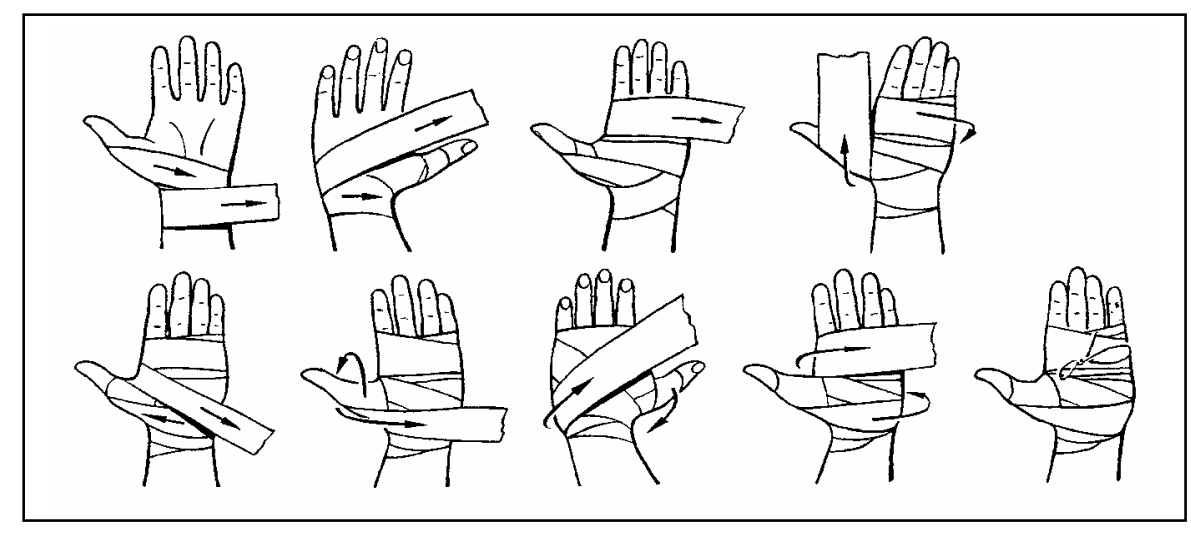

Abb. 4: Das Bandagieren der Hände (nach: FIEDLER, 1997, 19) 
Zur Benotung der Kämpfe werden Punktrichter eingesetzt, die wie folgt die verschiedenen Treffer und Aktionen bewerten:

- von den Handlungen des Gegners,

- von der Beherrschung der vielseitigen Technik,

- von dem taktischen Vermögen des Kämpfers,

- von dem Entwicklungsstand der Reaktion, der Ausdauer und weiteren speziellen und allgemeinen Konditionellen Fähigkeiten.

Computergestützte Punktwertungssysteme wurde zum ersten Mal während Boxweltmeisterschaften 1990 in Moskau eingeführt und befassten sich mit den Anforderungen, die diese Systeme an die Punktrichter stellt, wie die Schwierigkeiten in der Interpretation der Bewertung sowie den zu erwartenden Einfluss dieser Bewertungssysteme auf den Kampfstil und letztlich auch auf das Training. (vgl. REDO; TILEWSKI, 1990)

Bei den Amateuren besteht eine Einteilung in 12 Gewichtsklassen, bei dem Profibereich bereits 17 Gewichtklassen. (vgl. ROSS; CASSON u. a., 1987; MÜLLER, 1991; SONNENBERG, 1993; ELLWANGER; ELLWANGER, 1998)

Auf dem Wahlkongress in Kairo im Januar 2003 beschloss die AIBA, dass zukünftig beim olympischen Boxen die bisher 12 Gewichtsklassen auf 11 reduziert werden. Das Halbmittelgewicht wurde gestrichen und die Gewichtsspanne in den Limits Halbweltergewicht (bis $64 \mathrm{Kg}$ ) und Weltergewicht (bis $69 \mathrm{Kg}$ ) verändert. Hintergrund der Gewichtsklassen-Reduzierung war eine Vorgabe des (IOC) zur Verringerung der Anzahl der Medaillen. (http://www.boxensport.de/ Archiv/news archiv5.htm)

Die neue Gewichtsklassen-Struktur: Halbfliegengewicht (bis 48 kg), Fliegengewicht (bis $51 \mathrm{~kg}$ ), Bantamgewicht (bis $54 \mathrm{~kg}$ ), Federgewicht (bis $57 \mathrm{~kg}$ ), Leichtgewicht (bis $60 \mathrm{~kg}$ ), Halbweltergewicht (bis $64 \mathrm{~kg}$ ), Weltergewicht (bis $69 \mathrm{~kg}$ ), Mittelgewicht (bis $75 \mathrm{~kg}$ ), Halbschwergewicht (bis $81 \mathrm{~kg}$ ), Schwergewicht (bis 91 kg), Superschwergewicht (über $91 \mathrm{~kg}$ ). (siehe Tabelle in Anhang 10) (http://www.kontaktrunde.de/boxen/gewichtsklassen.html) 
Bei den Olympischen Spielen von Atlanta 1996 und den hier analysierten Spielen von Sydney 2000 gab es neben der Einteilung in zwölf Gewichtsklassen auch eine obere Altersgrenze von 34 Jahren. (vgl. SEKULES, 2000 )

Die Einführung des Frauenboxens wurde 1994 beschlossen. Beim Frauenboxen gibt es 16 Gewichtsklassen. Papiergewicht (bis $38 \mathrm{~kg}$ ), Papiergewicht (bis $44 \mathrm{~kg}$ ), Papiergewicht (bis $46 \mathrm{~kg}$ ), Halbfliegengewicht (bis $48 \mathrm{~kg}$ ), Fliegengewicht (bis $50 \mathrm{~kg}$ ), Bantamgewicht (bis $52 \mathrm{~kg}$ ), Federgewicht (bis $54 \mathrm{~kg}$ ), Leichtgewicht (bis $57 \mathrm{~kg}$ ), Halbweltergewicht (bis $60 \mathrm{~kg}$ ), Weltergewicht (bis $63 \mathrm{~kg}$ ), Halbmittelgewicht (bis $66 \mathrm{~kg}$ ), Mittelgewicht (bis $70 \mathrm{~kg}$ ), Halbschwergewicht (bis $75 \mathrm{~kg}$ ), Schwergewicht (bis $80 \mathrm{~kg}$ ), Superschwergewicht (bis $86 \mathrm{~kg}$ ), Superschwerplusgewicht (über $86 \mathrm{~kg}$ ). (siehe Tabelle in Anhang 11) (http://www.psvgeorgsmarienhuette.de/box geschichte.html) Da sich die vorliegende Arbeit mit dem olympischen Turnier der Männer befasst und noch immer wesentlich mehr Männer als Frauen boxen, wird immer die männliche Form (,,der Boxer”) verwendet, selbst wenn die technischen Beschreibungen in gleicher Weise meist auf Boxerinnen zutreffen.

Die Einteilung der Gewichtsklassen im Boxsport ist unterschiedlich geregelt und richtet sich nach der Einteilung in Amateur- oder Profibereich, im Profibereich wiederum nach der Zugehörigkeit zu einer der verschiedenen Profiboxsportweltverbände wie WBC, WBA, IBF oder WBO. (siehe Tabelle in Anhang 12) (vgl. STEWART; GARDEN u. a., 1994; ELLWANGER; ELLWANGER, 1998)

Ein guter Boxer muss neben einer hervorragenden Kondition über ein sehr hohes Fertigkeitsniveau verfügen, er muss alle Techniken und Taktiken beherrschen. (vgl. SCHULZ, 1984)

Die Boxtechniken bestehen aus den drei Grundschlägen: Die Geraden, Seitwärtshaken und Aufwärtshaken, die sich auf den Kopf und Oberkörperbereich beziehen, und vier Verteidigungsarten: Die Deckungen, Paraden, Meidbewegungen und Ausweichbewegungen. (vgl. FIEDLER, 1976; BASTIAN, 1978) 
Zuerst muss der Boxer die Boxstellung und die richtige Beinarbeit lernen. (vgl. FIEDLER, 1976; STAUFFER, 1993) Die Beseitigung von Bewegungs- und Handlungsfehlern sind im Ausbildungsprozess des Boxsports unerlässlich. Das trifft sowohl auf die sporttechnische als auch auf die sporttaktische Ausbildung zu. Bei der Fehlerkorrektur sind auf jeden Fall die Fehlerursachen zu ermitteln, um daraus differenzierte Folgerungen abzuleiten. Ursachen und Ableitungen beziehen sich dabei auf die Kenntnisse und das Können des jeweiligen Sportlers. (vgl. BÖTTICHER, 1952; BERGER, 1996; DENZ, 1997)

Der Boxer versucht immer mit diesen Boxschlägen und Verteidigungen zum Erfolg zu kommen. Jeder Kampf verläuft im Rahmen der taktischen Haupthandlungen von Angriff und Verteidigungen. (vgl. OGURENKO, 1972; STAUFFER, 1993; SONNENBERG, 1993)

Die Entwicklung des Boxens in den letzten zehn Jahren ist vor allem auf Änderungen zurückzuführen, die auf den Einsatz verschiedener technischer und taktischer Mittel sowie modifizierter Regelauslegungen zurückgehen. Ausgewertet wurden die Finalkämpfe der Olympischen Spiele 1988, die Weltmeisterschaften 1993 und die Olympischen Spiele 1996. Hervorgehoben wurde die steigende Anzahl von Verteidigungshandlungen. Mit der Einführung des elektronischen Kampfrichterwesens gibt es auch Veränderungen in der Taktik der Kampfesführung. Die Zahl der eingesetzten taktischen Varianten der Kampfentwicklung ist spürbar zurückgegangen, es wird auf ein Übergewicht in der Punktwertung mit Hilfe einzelner Schläge zu Beginn des Kampfes hingearbeitet. Insgesamt kann festgestellt werden, dass die Modernisierung der Technik zur höheren Sicherheit für die Boxer geführt hat. Ein Vergleich der K.O. Siege von 1988 und 1996 (28 zu 4) legt eindeutig Zeugnis ab vom Wandel im modernen Boxen. (vgl. DURJASZ; NOWAK, 1997)

Ein fortdauerndes zielgerechtes und individuell abgestimmtes Training der Reaktions-, Schlag und Bewegungsschnelligkeit hängt von ihrer Optimierung ab. Durch die Entwicklung eines speziellen Testgerätes wurden die Möglichkeiten zur Messung dieser Eigenschaften erreicht. Erste Untersuchungen mit Sportlern unterschiedlicher Leistungsklassen und unterschiedlichen Trainings- 
bzw. Wettkampfalters lassen durch die einzelnen Tests Zusammenhänge zwischen Wettkampfleistungen und dem Niveau der Schnelligkeitseigenschaften erkennen. (vgl. KIRCHGÄSSNER; WAGNER, 1971; KIRCHGÄSSNER; BASTIAN, 1984)

Eine Form von Boxtechnik und Taktik ist das Fintieren. Durch das Fintieren erfährt der Boxer viel über die Reaktionen, die Fertigkeit und das Kampfniveau seines Gegners. Daraufhin ist er in der Lage den Angriffsschlag auszuführen. Das Fintieren ist in jeder Runde in all seinen Variationen variabel zu handhaben und ermöglicht eine Vielfalt von Reaktionsmöglichkeiten und ist somit eine der wesentlichen Vorraussetzungen, die der Boxer beherrschen muss, um den Erfolgssangriff zu ermöglichen. Fintieren als eigene Kategorie ist jedoch schwer zu beobachten, da sie sowohl in die Angriffs-, Verteidigungs- als auch Abtasthandlung integriert ist.

Ich habe die Olympiade in Sydney 2000 ausgewählt, weil dies "die letzten" Olympischen Spiele bis heute sind. Außerdem nehmen die meisten der besten Boxer an den Olympischen Spielen teil, die somit mit einer Weltmeisterschaft zu vergleichen ist. Deshalb werde ich bei den Olympischen Boxwettkämpfen in Sydney im Jahre 2000 die einfachen und kombinierten Finten analysieren, um Aufschluss über die Art der von den Siegern beherrschten Finten zu erhalten und zu wissen, welche Arten von Finten am wirksamsten sind.

Die Olympischen Boxkämpfe- Sydney 2000 wurde von 16.September bis zum 01.Oktober, die Halbfinale am 28., 29.September, die Finale von 30.September. bis zum 01.Oktober ausgeführt. (siehe Tabelle in Anhang 2)

Wettkampfbeobachtungen wurden in den Ländern des ehemaligen Ostblocks schon lange durchgeführt. Erstmals wurde im Jahre 1971 bei den Europameisterschaften eine Wettkampfbeobachtung im Boxen in der Bundesrepublik Deutschland durchgeführt, die in den Schulungsplan für die Olympiavorbereitung München 1972 einfloss. In den Folgejahren wurde die Wettkampfbeobachtung mit unterschiedlichem Aufwand und unterschiedlichem Erfolg fortgesetzt. Seit ca. 1979 werden Informationen in der Boxbeobachtung mittels Videorecor- 
der gewonnen und in Beobachtungsbögen registriert. Diese Bögen umfassen alle wichtigen technischen und taktischen Daten des Boxsports, die Beurteilung der Kondition und der Psyche wird subjektiv ausgewertet. (vgl. JOHANNPETER, 1979)

Die olympischen Boxwettkämpfe in Seoul im Jahre 1988 bestätigen, wie konturenscharf sich heute im internationalen Boxsport die zwei grundlegenden Entwicklungstendenzen gegenüberstehen: das technische Boxen, gepaart mit der zum Erfolg notwendigen Physis der Sportler, und das sich immer stärker an das Profiboxen anlehnende harte, bisweilen auch unfaire aggressive Fighten, das in Seoul Dominanz erlangt hatte. Die unterschiedlichen Werturteile spiegeln sich auch bei dem Kampf- und Ringrichtern wider. Der DBV der DDR wurde in seiner Ansicht bestärkt, dass sich weiterhin auch technisch versierte Boxer mit der notwendigen Physis erfolgreich durchsetzen können, wenn auf dem Wege individualisierter Ausbildung das Deckungsverhalten und vor allem die allgemeine und spezielle athletische Ausbildung noch enger mit der technischtaktischen Ausbildung verbunden werden kann. Die Tatsache, dass durch ein Bündel von Entscheidungen der AIBA nunmehr Kontinental- und Weltmeisterschaften innerhalb eines Jahres zu bewältigen sind, setzte die Frage nach der trainingsmethodischen Periodisierung neu auf die Tagesordnung. (vgl. FIEDLER, 1989)

An positive Tendenzen des Boxsports anknüpfend, war nach den extremen Entgleisungen bei den olympischen Boxwettkämpfen und bei den Weltmeisterschaften ein deutlicher Aufwärtstrend zu erkennen. Dazu trugen vor allem die Boxsportler Kubas, der UdSSR und der DDR bei. Durch ihre leistungsmäßige Dominanz prägten sie wesentlich das sportliche Niveau. Unter dem Druck der Ereignisse von Seoul und dem Drängen des IOC forcierte die AIBA Maßnahmen für den Gesundheitsschutz der Boxsportler und die weitere Qualifizierung der Kampfrichter. Unter diesem Aspekt ist auch der Einsatz der Punktmaschine bei den Weltmeisterschaften einzuordnen. Im Trend zeichnet sich eine stärkere Hinwendung zum technischen Boxen mit geringerer Härte ab. Analysen weisen auf eine weitere Erhöhung der Handlungsdichte und eine Vereinfachung der Angriffshandlungen hin. (vgl. FIEDLER; SCHULZ, 1990) 
In Vorbereitung auf die Olympischen Spiele ist in der Vorbereitungsperiode die Stabilisierung der allgemeinen physischen Kondition erforderlich, wobei das Hauptaugenmerk auf die spezielle und individuelle körperliche Vorbereitung gelegt wird sowie auf die Vervollkommnung der individuellen taktisch-technischen Kampfweise. In der Hauptperiode soll an der Stabilisierung der individuellen psycho-physischen und taktisch-technischen Vorbereitung gemäß den Besonderheiten der einzelnen Boxer und ihrer erwarteten Hauptgegner gearbeitet werden. (vgl. ANFREEV, 1996)

Die prognostizierte Leistungsentwicklung in den Zweikampfsportarten stellt höchste Anforderungen an die spezifischen Schnelligkeitsleistungen. Anhand von sportartspezifischen Tests, Wettkampf- und Trainingsanalysen insbesondere in der Etappe des Aufbautrainings wurden seit 1984 die Schnelligkeitsleistungen dementsprechend angepasst. (vgl. KIRCHGÄSSNER; BASTIAN, 1984)

Fortschritte in der technisch- taktischen Verbesserung des Boxens spiegeln sich vor allem in den Altersklassen ab 14 Jahren und in den folgenden Klassen, etwas differenzierter wieder. Eine weitere Verbesserung wird mit der Individualisierung zum Ende des Aufbautrainings erreicht. Zudem bestehen noch Reserven in der konsequenten Ausrichtung des Trainings in den einzelnen Etappen auf die darauf folgende Etappe. (vgl. BRAUSKE; SCHLIMPER, 1988)

Im sportlichen Training spielt die Kondition eine wesentliche Rolle:

a) im Sinne einer allgemeinen Fitness durch die Ausbildung motorischer Grundeigenschaften,

b) als sportartspezifische Leistungsfähigkeit durch die Entwicklung spezieller motorische Fähigkeiten. Diese motorischen Fähigkeiten sind Voraussetzungen für das Erlernen technischer Fertigkeiten, definieren das Konditionstraining in seiner Funktion, seinen Aufgaben und seinem Inhalt. Die Konditionseigenschaften Ausdauer, Kraft, Schnelligkeit, Koordination, Beweglichkeit und Willenseigenschaften sind Leistungsparameter und Haupttrainingsform. Das Übungsprogramm ist aufgrund der systematisch gegliederten Übungsformen das Standardprogramm für das allgemeine Konditionstraining. (vgl. HOWALD, 1985; 
KOMI; HÄKKINEN, 1989; ISRAEL, 1994; KNUTTGEN; KOMI, 1994; KOCH, 1997; KRAMPE; KLIEGL u. a., 2000)

Techniken wurden bereits wissenschaftlich untersucht und gegenwärtig liegen nur wenige Untersuchungen von Techniken und Taktik beim Boxen vor. (z. B. JONATH; KREMPEL, 1985; KIRCHGÄSSNER; BASTIAN, 1984; FIEDLER, 1974; LATYSENKO; RODIONOV, 1977; ROMANOV; BAJKOV, 1978; KULIEV, 1981; VALENTINO; ESPOSITO u. a., 1990; JOST; WEISS u. a., 1994) (siehe Kapitel 3 in 3.1)

In der ältesten durch Literatur abgesicherten Zweikampfsportart Fechten (KRÜGER, 1994) haben Finten schon immer (SCHENKER 1961, 154 - 161) zum Ausbildungsprogramm gehört. Auch für BECK $(1978,105 f f$.$) gehören Finte$ und Fintangriff zu den wesentlichen Lektionen der Spitzenfechter. BARTH $(1995,22)$ unterscheidet in diesem Zusammenhang zwischen Fintangriff und KontraPriposte, die er aber als Beispiele für alle Zweikampfsportarten versteht.

Das Fintieren als Strategie im Boxen wurde bis heute noch nicht ausführlich empirisch untersucht. Aus diesem Grund werden im ersten Teil der Arbeit bisher erforschte Erkenntnisse zu Täuschungshandlungen im Sport angeführt und die Bedeutung von Finten im Sport dementsprechend eingeordnet. Der zweite Teil der Arbeit beinhaltet schließlich die Analyse von Amateurboxern aller Klassen in Hinsicht auf ihr Fintierverhalten bei den Olympischen Spielen 2000 in Sydney.

\subsection{Zielsetzung}

Die vorliegende Studie soll uns folgende Fragen beantworten:

1- Welche Arten von Finten beherrschen die Sieger?

1- Welche Finten sind am wirksamsten?

2- Welche Gewichtsklassen verwendet Finten am häufigsten?

3- Welche Arten von Finten dominieren in den Runden? 
4- Welche nationalen Boxschulen sind erfolgreich?

5- Wie können die Boxer und die Finalboxer der Meisterschaft im Hinblick auf ihre Finten charakterisiert werden?

\subsection{Inhaltliche Hypothesen}

H.1. Der Sieger verwendet mehr Finten als der Verlierer.

H.2. Der Sieger verwendet mehr kombinierte Finten als der Verlierer.

H.3. Der Sieger verwendet mehr variable kombinierte Finten als der Verlierer.

H.4. Schwere Boxer verwenden Finten häufiger als leichte Boxer.

H.5. Schwere Boxer verwenden kombinierte Finten häufiger als leichte Boxer.

H.6. Der Sieger macht gegen Ende des Kampfes häufiger variable Finten als der Verlierer.

H.6.1. Schwere Sieger machen gegen Ende des Kampfes häufiger variablere Finten als leichte.

H.6.2. Schwere Boxer machen gegen Ende des Kampfes häufiger variable Finten als leichte.

H.7. Die Sieger variieren die Art der Finten von Runde zu Runde stärker als die Verlierer.

H.8. Es gibt erfolgreiche nationale Boxschulen, die sich mit einem eigenen Fintierstil darstellen. Die Boxer aus USA, RUS, UKR, CUB und KAZ unterscheiden sich in ihren Fintiermustern.

H.8.1. Die Amerikanische Boxschule unterscheidet sich von denen aus RUS, UKR, CUB und KAZ.

H.8.2. Die RUS Boxschule unterscheidet sich von der aus UKR, CUB und KAZ.

H.8.3. Die CUB Boxschule unterscheidet sich von der aus der UKR und KAZ.

H.8.4. Die KAZ Boxschule unterscheidet sich von der aus der UKR. 


\subsection{Definitionen von Täuschungshandlungen im Sport}

OKONEK (1987) hat sich systematisch mit Täuschungshandlungen im Sport am Beispiel von Basketball befasst. Demnach lassen sich alle Täuschungshandlungen im Sport den Kategorien Lüge, Täuschungsaufstellung, Betrug, Bluff, Finte und Imitationstäuschung zuordnen. Die hier vorgenommene Gliederung stellt die Arbeitsgrundlage für die später erfolgende theoretische Betrachtung dar.

Definition 1: Die Finte ist eine wettkampfinterne versuchte, vorsätzliche Irreführung mittels nonverbaler und in Bedingtheit durch die Zeit lückenhafter Information. Die Finte soll den unter Zeitdruck stehenden Konkurrent zu falscher Ziel- und Programmantizipation und damit zu einer raum-zeitgerecht nicht mehr reversiblen open-loop-Bewegung veranlassen (vgl. BÖS, 2003); Beispiel: Wurffinte im Basketball, Handball, Boxen etc.

Definition 2: Die Imitationstäuschung ist eine versuchte, vorsätzliche Irreführung einer sachlichen Schiedsinstanz zum Vorteilsgewinn gegenüber dem Konkurrent mittels nonverbaler, gänzlicher und verfälschter Information; Beispiel: 'Schwalbe' im Fußball, um 'Elfmeter' zugesprochen zu bekommen.

Definition 3: Die Täuschungsaufstellung ist eine wettkampfinterne versuchte, vorsätzliche Irreführung mittels nonverbaler, gänzlicher und echter Information einer Person. Sie soll den Konkurrent zur Wahl taktisch unangemessener Handlungen veranlassen; Beispiel: Fintenaufstellung im Volleyball mit Scheinläufer und/oder Scheinangreifer.

Definition 4: Der Bluff ist erklärt im Rahmen der mathematischen Spieltheorie als Gefahrstrategie im Poker in der Differenz zum konservativen Spiel. Bluffs sind vorsätzliche Irreführungen mittels mittelbarer verbaler oder non-verbaler Information und haben zum Ziel, den Konkurrenten in seiner Strategiewahl zu beeinflussen. Im Sport werden dabei nur die Grundsätze des Bluffens angewandt:

1. Fingieren von Stärke bei faktischer Schwäche und vice versa. 
2. Der Konkurrent soll entweder verunsichert oder in falscher Sicherheit gewiegt werden. (vgl. NEUMANN; MORGENSTERN, 1974; vereinfachte Darstellung vgl. z.B. THIE, 1979)

Definition 5: Die Lüge ist eine versuchte vorsätzliche Irreführung anderer mittels manipulierter Information. Eine Person A hat gelogen, genau dann, wenn

1. A hat behauptet, dass $p$

und

2. A weiß, dass nicht $p$.

Lügen sind gekennzeichnet durch fünf Eigentümlichkeiten, sie sind

1. personal (jemand lügt),

2. gesellschaftlich (jemanden betreffend),

3. temporal (exakt datierbar),

4. absichtlich und

5. verbal (laut- oder schriftsprachig)

(vgl. FALKENBERG, 1982, in Anlehnung an CHISHOM; FEEHAN, 1977). Diese sprachwissenschaftliche Definition gilt auch für den Sport und wird z. B. bei Befragen durch den Schiedsrichter angewandt.

Definition 6: Betrug ist ein rechtlich definierter Begriff. Der Tatbestand des Betruges gilt als erfüllt, wenn die folgenden vier Tatbestandsmerkmale vorhanden sind, unabhängig davon, ob der Betrug mit Vorsatz oder aus Fahrlässigkeit heraus begangen wurde:

1. Vorspiegelung falscher oder Unterdrückung oder Entstellung wahrer Fakten,

2. Irrtumserregung beim Opfer,

3. Vermögensverfügung des Getäuschten und

4. Vermögensschaden beim Getäuschten.

(vgl. LACKNER, 1995; SCHÖNKE; SCHRÖDER, 1991) Der Begriff hat in diesem Verständnis auch im Sport Gültigkeit. Doping im Sport erfüllt bewusst meist den Tatbestand des Betruges. 


\section{Training im Boxkampf}

\subsection{Techniktraining im Boxen}

Der Begriff des Techniktrainings scheint eindeutig bestimmt, nämlich als Training sportartspezifischer Techniken. Das Techniktraining bezeichnet die Gesamtheit derjenigen Maßnahmen und Verfahren, die dazu dienen, die Techniken mit ihren sportmotorischen Fertigkeiten, deren Anwendung sowie technischen Einsatz systematisch zu erlernen und in Boxkampfsituation optimale Erfahrungen zu sammeln. (vgl. MARTIN, 1991; HOHMANN, 1996; HOHMANN; LAMES u. a., 2002)

Beträchtliche Auffassungsunterschiede ergeben sich allerdings, wenn es um die genauere Bestimmung des hier eingeschlossenen Technikbegriffs geht. In den vorliegenden Definitionen finden sich insbesondere folgende unterschiedlichen Akzentsetzungen:

1. Technik ist eine spezifische Abfolge von Bewegungen oder Teilbewegungen beim Lösen von Bewegungsaufgaben in Boxkampfsituationen. (vgl. MECHLING; CARL, 1992)

2. Technik als anforderungsspezifische Bewegungskoordination (innere Bewegungsstruktur), insbesondere auf der Grundlage von Informationsverarbeitungsprozessen. (vgl. DAUGS, 1988; HOHMANN,1997)

Für den Boxsport ist die anforderungsspezifische Bewegungskoordination der inneren und äußeren Bewegungsstruktur als die allgemein gesehene bestimmungsgebende Definition des Technikbegriffs.

Die theoretischen Grundlagen des Techniktrainings stammen aus einer ganzen Reihe von Basis-Wissenschaften. Physiologie, Biomechanik, Motorik und Psychologie liefern beispielsweise - je in ihren Domänen - Beiträge, die von der Trainingswissenschaft im Hinblick auf eine wissenschaftliche Fundierung des Techniktrainings integriert werden müssen. Die dabei auftretenden Schwierigkeiten sind zahlreich: 
Wissenschaftstheoretisch ist ein echtes interdisziplinäres Vorgehen generell als Ausnahmefall anzusehen, natürlich limitierte Ressourcen erlauben dem Trainingswissenschaftler kaum die Rezeption aller Informationen auf den relevanten Feldern und schließlich ist zu beobachten, dass der theoretische Erkenntnisstand keine unidirektional und stetig wachsende Größe darstellt, sondern die Entwicklung dort von Modeströmungen, Paradigmen-Wechseln und auch von umwälzenden Erkenntnissen geprägt ist. (vgl. FARRELL, 1975; HOHMANN; LAMES u. a., 2002)

Das für das Techniktraining elementare Lernproblem ist nicht zuletzt deshalb ein offenes Problem, weil Annahmen über das motorische Lernen immer auf Annahmen über die Organisation der Motorik beruhen. Die Methodik des Techniktrainings müsste stark differieren, wenn es auf der Basis einer ProgrammTheorie oder eines Selbstorganisationsansatzes konzipiert wird. Im ersten Fall würde man beispielsweise versuchen, Teilbewegungen zu einer Gesamtbewegung zu synthetisieren. Im zweiten Fall ginge es darum, die Umwelt des Lernenden so zu organisieren, dass Selbstorganisationsprozesse induziert oder Gelegenheiten zur Herausbildung von Bewegungsstrukturen geschaffen werden.

Wenn es angesichts der aufgezeigten Komplexität des Techniktrainings eine Aussage zu treffen gilt, die als Grund des Techniktrainings verstanden werden kann, dann muss auf die Vielfalt an Trainingszielen hingewiesen werden. Die praktische Begründung dafür liegt in dem Umstand, dass je nach verfolgtem Trainingsziel im Techniktraining andere, oft gegensätzliche Bedingungen zu schaffen sind, um ein optimales Resultat zu erreichen. (vgl. MARTIN, 1977; LETZELTER, 1978; GROSSER, NEUMEIER, 1982; HOHMANN; LAMES u. a., 2002)

Der Grundsatz des Techniktrainings besteht in der Beachtung des Spektrums seiner Ziele, da je nach Ziel trainingsmethodisch, sogar gegensätzlich vorgegangen werden muss. Es werden vier Zielkategorien unterscheiden:

1. Das Erlernen von Fertigkeiten (Technikerwerbstraining): Neulernen technischer Fertigkeiten bis zur Automatisierung des dynamischen Optimums, 
2. Das Variieren von Fertigkeiten (Technikvariationstraining): Erlernen von Varianten technischer Fertigkeiten bis zum situationsgerechten Einsatz der Varianten,

3. Das Anpassen von Fertigkeiten (Technikanpassungstraining): Anpassen der Fertigkeiten an Raum (räumliche Konstellation) und Zeit (schneller Wechsel von Kampfbedingungen),

4. Das Abschirmen von Fertigkeiten (Technikabschirmungstraining): Stabilisieren oder Abschirmen von Fertigkeiten gegen Gegnereinfluss oder Konditionelle Belastungen.

Es sei nur angedeutet, dass der Erwerb technischer Fertigkeiten im Boxsport sicherlich auf jedem Niveau anzutreffen ist und dass beim Boxsport mit geschlossener Aufgabenstruktur, also mit konstanten Bedingungen und ohne direkten Gegnereinfluss, die Zielkomplexe der Anpassung und der Abschirmung gegen Gegnereinwirkung naturgemäß entfallen. (vgl. WILLIMCZIK; ROTH, 1983; HARRE, 1986; HOHMANN, 1994; 1996; 1997; 1999a; 1999b; HOHMANN; LAMES, 2002)

\subsection{Elementares Schnelligkeitstraining im Boxen}

Die elementare Schnelligkeit hängt davon ab, wie gut die Steuer- und Regelmechanismen des Zentralnervensystems und des Nerv-Muskel-Systems funktionieren und zusammenspielen. Die Sequenz- und Frequenzschnelligkeit sind damit - wie im Übrigen die primär sensorisch-kognitiv sowie psychisch bedingte (einfache) Reaktionsschnelligkeit auch - in hohem Maße anlage- und reifebedingt. Die für sportliche Spitzenleistungen erforderliche Qualität der zentralnervösen und neuromuskulären Steuerung zeigt sich darin, dass ein Boxer sowohl bei zyklischen als auch azyklischen Bewegungen über eine schnelle Bewegungsausführung verfügt.

Die elementaren Schnelligkeitsfähigkeiten im Boxen entwickeln sich im Verlauf des Kindes- und Jugendalters positiv ansteigend (vgl. WINTER, 1976; HOHMANN, 1996). Optimale Ausprägungen können dann erreicht werden, wenn sie 
gezielt mit Hilfe eines elementaren Schnelligkeitstrainings und frühzeitig, also bereits in der vorpuberalen Phase geschult werden. Durch das "normale“, d. h. überwiegend Kraft- und Ausdauerorientierte Training im Nachwuchsalter lassen sich die elementaren Schnelligkeitsanlagen nur selten voll entfalten. Insofern bestehen hier trainingsmethodische Reserven, um die perspektivischen Schnelligkeitsleistungen zu optimieren. Im Kindes- und Jugendalter sind die Kompensationsmöglichkeiten zwischen den elementaren Leistungsvoraussetzungen noch vielfältig. Insofern ist beim Training von Nachwuchsboxern von besonderer Bedeutung, dass

- die (einfache) Reaktionsschnelligkeit,

- die azyklische Sequenz und

- die zyklische Frequenz

getrennt beurteilt und angesteuert werden. In der Boxpraxis werden folgende und ähnliche Bewegungsanforderungen zur Ausbildung der elementaren Schnelligkeit eingesetzt:

- Azyklische Sequenzschnelligkeit: Nieder-Hoch-Sprung, (angefallener und geprellter) Liegestütz aus dem Kniestand, (leicht angefallener und geprellter) Wandstütz aus dem Stand, Unterarmdrehung (Supination), Fallstabübung;

- Zyklische Frequenzschnelligkeit: Finger-, Hand- und Armtapping, Beintapping (im Stehen, Sitzen, in Bauchlage mit dem Rist, in Rückenlage gegen die Wand), Armkurbeln, Beinkurbeln.

Die zwei vorgenannten Trainingsmethoden sind insbesondere für die Finten mit den Armen, den Beinen und die kombinierten Finten wichtig. (vgl. HOHMANN, 1997; HOHMANN; LAMES, 2002)

\subsection{Komplexes Schnelligkeitstraining im Boxen}

Vielfach belegt ist der überragende Einfluss der komplexen Schnelligkeitsfähigkeiten

- komplexe (großmotorisch) Reaktionsschnelligkeit,

- azyklische Aktionsschnelligkeit und

- zyklische Bewegungsschnelligkeit 
auf die Leistung im Boxsport. Die komplexe Reaktionsschnelligkeit lässt sich in der Regel durch ein Reaktionstraining eher verbessern als elementare, einfache Reaktionsleistungen. Der erreichbare Leistungszuwachs korrespondiert bei Einfachreaktionen mit der Zunahme des Bewegungsumfangs und bei komplexen Wahlreaktionen, wie sie im Boxsport vorkommen, mit der Zahl der Handlungsalternativen. Je schneller eine spezifische Reaktion ausgeführt werden kann, umso mehr verlagert sich der Trainingseffekt von einer Reaktionszeitverkürzung hin zu einer Konstanz der Reaktionszeit. Dies geschieht über die Automatisierung und Stabilisierung des motorischen Anteils an der Reaktionszeit. Der Verbesserung der weiteren informatorischen Anteile am Reaktionsablauf sind sehr enge Grenzen gesetzt. (vgl. KRÜGER, 1982a; HOHMANN; LAMES, 2002) LEHMANN $(2000,108)$ weist auf den engen Zusammenhang von steigender konditioneller Belastung und Reaktionszeitwerten hin und fordert, dieses durch entsprechendes Ausdauertraining zu optimieren.

\subsection{Beweglichkeitstraining im Boxen}

Im Vergleich zur Gelenkigkeit ist die Dehnfähigkeit der Muskeln, Sehnen und Bänder besser zu trainieren. Allerdings wird das Ausmaß der möglichen Leistungsverbesserung meist überschätzt. Trainingspraktische und experimentelle Erfahrungen zeigen, dass deutliche Beweglichkeitsgewinne nur durch ein langfristiges regelmäßiges (d. h. nahezu tägliches) und intensives Training zu erreichen sind. Mit der in vielen Sportarten üblichen „Aufwärmgymnastik" sind nachhaltige Bewegungsverbesserungen kaum zu erzielen. Diese hat eigene Ziele, die nicht mit denjenigen des Beweglichkeitstrainings verwechselt werden dürfen.

Neben einer Reihe von äußeren und weiteren inneren Einflussfaktoren spielen die neuromuskulären Bedingungen bei der Dehnfähigkeit die größte Rolle und liegen direkt oder indirekt allen Zielen und Methoden des Beweglichkeitstrainings zu Grunde. Die früher sehr verbreitete Form der aktiv dynamischen Schwunggymnastik (federndes oder aktiv dynamisches Dehnen) findet heute im Beweglichkeitstraining kaum noch Anwendung. Der Grund dafür ist, dass bei 
der schwunghaften Dehnung eines Muskels Eigenreflexe ausgelöst werden, die für eine sofortige Kontraktion des Muskels sorgen. Diese monosynaptischen Reflexe werden durch im Muskel eingelagerte Muskelspindeln ausgelöst und dienen dem Muskel als Schutzmechanismus vor Verletzungen (Zerrung, Muskelfaserriss). In diesem Fall ergibt sich bei der nachfolgenden (konzentrischen) Muskelaktion eine höhere Anfangskraft. (vgl. CRATTY, 1975; FARRELL, 1975; KNAPP, 1977; WIEMANN, 1991)

Zur wichtigsten Methode in Beweglichkeitstraining ist die gehaltene Dehnung oder das Stretching geworden. Dabei wird die Muskulatur langsam bis kurz vor die Schmerzgrenze aufgedehnt und in dieser Position 20-30 s lang gehalten. Die dazu erforderliche Kraft wird bei der aktiven statischen Dehnung durch die Kontraktion der antagonistischen Muskulatur und bei der passiven statischen Dehnung durch die Schwerkraft aufgrund des eigenen Körpergewichts oder durch die Zugunterstützung seitens der Arme erzeugt. Gegebenenfalls kann auch ein Trainingspartner oder ein äußerer Widerstand bei der Muskeldehnung unterstützend einwirken. Wesentlich effektiver, dafür jedoch auch stärker belastend, ist die Anspannungs-Entspannungs-Dehnung, auch CHRS-(contract-holdrelax-stretch-)Dehnung genannt. Bei dieser Methode wird der eigentlichen Dehnphase von 20-30 s eine 6-10 s dauernde Anspannungsphase mit anschließender kurzer Entspannung des zu dehnenden Muskels vorgeschaltet. Dabei kann die Dehnung sowohl aktiv über die Antagonistenkontraktion als auch passiv über das Eigengewicht des Boxers vorgenommen werden. Der Vorteil der Anspannungs-Entspannungs-Dehnung gegenüber dem herkömmlichen Stretching liegt darin, dass die angestrebte Dehnung der die Muskelfasern umgebenden Bindegewebsstrukturen durch die einleitende Anspannung des Muskels deutlich verstärkt wird. Dies geschieht, indem die kollagenen Fasern des elastischen Bindegewebes durch die Kontraktion aus einer eher ungeordneten in eine eher parallele Anordnung ausgezogen werden. Die anschließende Muskeldehnung in der Stretchphase findet somit unter wesentlich günstigeren muskulären Voraussetzungen statt. Mittel- und langfristig führt das Stretching zu strukturell-plastischen Anpassungsvorgängen beim kollagenen Material und damit zu einer dauerhaften Verbesserung der Beweglichkeit. (vgl. ULLRICH; GOLLHOFER, 1994; HOHMANN; LAMES, 2002) 


\subsection{Koordinationstraining im Boxen}

Koordinative Fähigkeiten sind einzelne Aspekte der Bewegungssteuerung, die in der Qualität ihrer Ausführung als überdauernde Verhaltensdispositionen betrachtet werden.

Für boxsportliche Zwecke wurde das Fähigkeitsmodell der Koordination immer schon als viel zu wenig ausdifferenziert betrachtet. Hier wurden Systematiken entwickelt, die spezifische Fähigkeiten enthalten wie zum Beispiel das Fintieren. (vgl. KOCH, 1997; HOHMANN; LAMES, 2002)

Es bleibt also festzuhalten, dass die klassischen koordiniativen Fähigkeiten derzeit den Status empirisch nicht eindeutig belegbarer, heuristischer Konzepte besitzen. Ihre Bedeutung ist daher vor allem im Sinne eines praktischen Leitfadens für die motorische Grundausbildung beispielsweise an der Schule zu sehen, dessen Einhaltung eine vielseitige und umfassende motorische Förderung absichern kann (vgl. HIRTZ, 1985). Die Tests für Koordination sind im Wesentlichen dem Turnen entnommen und entsprechen weder den Erforderwissen der Zweikampfsportarten noch den Sportarten mit beweglichem Untergrund z. B. dem Surfen. (HEISE, 1998)

Aus der Analyse des koordinativen Anforderungsprofils des Boxsports werden Maßgaben für das Koordinationstraining abgeleitet. Als Analysekategorien werden Informationsanforderungen und Druckbedingungen untersucht. Zur Analyse des koordinativen Anforderungsprofils sind dann die einzelnen Anforderungen und Bedingungen jeweils auf einem Kontinuum zwischen "hoch“ und "niedrig“ einzustufen. (vgl. NEUMAIER, MECHLING, 1994; 1995 ; NEUMAIER, 1999)

Da es sich bei der Koordination um Qualitäten der Bewegungssteuerung handelt, sind als Adaptationsmechanismus Selbstorganisationsprozesse der menschlichen Informationsverarbeitung anzunehmen. Um diese zu beeinflussen, kann man ein Prinzip des Koordinationstrainings formulieren, das nur auf den ersten Blick eine triviale Aussage darstellt:

Der Grundsatz des Koordinationstrainings besteht darin, dass der Trainierende koordinativ beanspruchende Übungen realisiert. 
Die Inhalte des Koordinationstrainings sollen im Idealfall also so gestaltet werden, dass der Trainierende an die Grenze der Leistungsfähigkeit seiner Informationsverarbeitung geführt wird, um die Selbstorganisationsprozesse zu induzieren, die zu einer Verbesserung der Qualität der Bewegungssteuerung führen.

Die Realisierung des Prinzips des Koordinationstrainings setzt zwei Bedingungen voraus: Der Trainer muss einmal über ein großes Reservoir koordinativ anspruchsvollen Übungsgutes verfügen und in der Lage sein, spontan Modifikationen von Übungen vorzunehmen, die den Schwierigkeitsgrad nach Bedarf anpassen. Darüber hinaus ist aber auch die Qualifikation erforderlich, die Beanspruchung der informationsverarbeitenden Systeme des Trainierenden durch die realisierten Übungen zu jedem Zeitpunkt korrekt zu erkennen.

Über den Anteil des Koordinationstrainings in den einzelnen Phasen des Trainingsaufbaus besteht keine einhellige Auffassung. Während generell im Grundlagenbereich eine Schulung der Koordination auf einer vielseitigen, „polysportiven" (HOTZ, 1993, 20) Basis erfolgen sollte, ist dies im Leistungsbereich umstritten. HIRTZ (1994) zitiert drei Auffassungen, nach denen

1. ein Koordinationstraining neben dem Techniktraining obsolet ist,

2. das Training spezieller koordinativer Fähigkeiten unter das Techniktraining subsumiert wird (technisches Ergänzungstraining nach MARTIN, 1991) und

3. das Koordinationstraining einen eigenständigen Bestandteil des Hochleistungstrainings ausmacht.

\subsection{Koordination und Techniktraining im Boxen}

Die Fähigkeit, Bewegungen mit hoher Qualität auszuführen, ist von großer Bedeutung im sportlichen Kontext. Im Boxsport sind Koordination und Technik sehr wichtig. Sie sichern die optimale Ausnutzung der energetischen Potenzen und die Ökonomisierung des Bewegungsablaufes, in dem Boxkampf dienen sie der taktisch vorgegebenen Zielerreichung.

In der Trainingswissenschaft war im Auf und $A b$ der Forschungsthemen in den späten 1980er und frühen 1990er Jahren des letzten Jahrhunderts, eine 
"Boomphase“ (WILLIMCZIK, 1991) der Befassung mit Themen des Techniktrainings zu registrieren. MARTIN, CARL und LEHNERTZ $(1991,43)$ attestieren demzufolge dem Techniktraining eine „übergeordnete Bedeutung“. Selbst wenn man nicht zu Verabsolutierungen neigt und berücksichtigt, dass eine Prioritätensetzung unter den Leistungsvoraussetzungen nur sportartspezifisch sinnvoll ist, sind die genannten Argumente durchaus schlüssig:

- Die „Eintrittskartenfunktion“: Nur wer über ein Minimum an technischen Fertigkeiten verfügt, kann am Boxsport teilnehmen.

- Die „Integrationsfunktion“: Zwar stellt jede Bewegung in der Regel Anforderungen an sämtliche Voraussetzungskomplexe (Ausdauer-, Kraft-, Schnelligkeitsfähigkeiten), die technische Fertigkeit integriert diese Verhaltensdispositionen jedoch erst zu einer boxsportlichen sinnvollen Bewegung.

Eine Bilanz der Ausführungen zum Koordinations- und Techniktraining muss konstatieren, dass das theoretische Grundlagenwissen aus den Basis- Wissenschaften bei weitem nicht ausreicht, um praktisches Handeln nachhaltig zu begründen. Auf der anderen Seite ist offensichtlich, dass an vielen Stellen der Boxpraxis erfolgreiches Techniktraining praktiziert wird. Aus diesem Grund liegt es besonders nahe, mit einer anderen Forschungsstrategie Wissen zum Techniktraining zu generieren. In der DDR wurde hierzu schon seit Beginn der 1960er Jahre systematisch zum Techniktraining in den Zweikampfsportarten in enger Zusammenarbeit mit der Sportpraxis geforscht. Die Arbeiten wurden hierbei immer disziplinspezifischer (MÜLLER-DECK, 2003). Für das Techniktraining im Boxsport wurde diese Strategie von der Arbeitsgruppe um Roth auch in Westdeutschland umfassend realisiert (vgl. ROTH, 1996). Dieses Projekt wird im Folgenden wegen der Ergebnisse und weil es als methodologisch wichtig für die Trainingswissenschaft betrachtet wird, kurz geschildert.

Unter Einsatz qualitativer Forschungsmethoden wurde versucht, die Alltagstheorien erfolgreicher Boxtrainer zu rekonstruieren. Ein Leitfaden-Interview mit Boxtrainern erhob ein Kategoriensystem wesentlicher Aspekte des Techniktrainings. In einer ersten Verdichtungsstufe wurde versucht, die Aussagen zu verschiedenen Kategorien zusammenzufassen. (vgl. HOHMANN; LAMES, 2002) 
Die erste Verdichtungsstufe ist für den fortgeschrittenen Praktiker sicherlich die aufschlussreichste, wird hier doch mitgeteilt, wie ein spezielles Problem in der Praxis des Boxsports angegangen wird. So wird beispielsweise deutlich, dass psychologisches Training im Boxsport nur selten von professionellen Sportpsychologen durchgeführt wird, sondern die Trainer wesentliche Aufgaben des psychologischen Trainings als ihr Tätigkeitsfeld begreifen: die Verbesserung von Bewegungsvorstellungen, die Abschirmung der Fertigkeit gegen psychischen und physischen Stress und die Sicherung und Steigerung der Konzentrationsfähigkeit im Boxkampf. Methodische Maßnahmen zu Erreichung dieser Ziele sind:

- das Training unter Wettkampfbedingungen, womit psychische und physische Belastungen simuliert werden,

- das Training unter erschwerten Bedingungen, mit denen Entscheidungs-, Zeitund Präzisionsdruck erzeugt werden, und

- das Training unter motivationsfördernden Bedingungen, das vor allem das Ziel verfolgt, unmittelbar vor einem Wettkampf das Selbstvertrauen der Sportler zu stärken. (vgl. HOHMANN, 1996; HOHMANN; LAMES, 2002)

\subsection{Training der kognitiven Fertigkeiten im Boxen}

Aus der denkbaren Anzahl kognitiver Fertigkeiten haben sich besonders sechs (zumindest aus pragmatischer Sicht) als bedeutsam und trainierbar herausgestellt. (vgl. MAHONEY, 1979; ORLICK; PARTIGTON, 1988; VEALEY, 1988; GROVE; HANRAHAN, 1988; EBERSPÄCHER, 1990) Diese Kognitiven Fertigkeiten und Methoden sind:

1. Selbstgesprächsregulation, monologisch, dialogisch (RUSHALL; HALL u. a., 1988). Die Methoden hierbei sind das Üben von anforderungsrelevanten Selbstgesprächen, Stimmungswörter.

2. Aufmerksamkeitsregulation, Konzentration, Distraktion (MAXEINER, 1988) und die Methoden sind Konzentrationstraining, Drehbuch, Umschalten.

3. Aktivationsregulation, relaxierend, mobilisierend (RENZLAND; EBERSPÄCHER, 1988) die Methoden sind Relaxations- und Mobilisationstechniken. 
4. Vorstellungsregulation (FELTZ; LANDERS, 1983) die Methoden sind Nichtmotorische Trainingsformen.

5. Self-Efficacy (BRODY; HATFILD u. a., 1988) die Methoden sind Prognosetraining, Nicht-Wiederholbarkeitstraining.

6. Analyse Zielsetzung (VEALEY, 1988) die Methoden sind Sensibilisierung und Wahrnehmungsschulung, realistische Kausalattribuierung, realistische Selbsteinschätzung.

In diesem Zusammenhang sind für Trainer und Boxer die wichtigsten kognitiven Fertigkeiten: Selbstgesprächsregulation, Selbstwirksamkeitsüberzeugung, Aufmerksamkeitsregulation, Aktivationsregulation, Vorstellungsregulation, Zielsetzung sowie die Analyse.

\subsection{Taktische Kenntnisse im Boxtraining}

Taktische Kenntnisse beziehen sich auf Regeln des Wettkämpfens, die offiziellen Wettkampfregeln sowie strategisch-taktische Regeln. Bei Anfängern und im nicht-leistungssportlichen Taktiktraining stehen zu Beginn die moralischen Regeln des Fair Play und des partnerschaftlichen Miteinanders im Wettkampf, sowie ganz allgemein das Sportethos im Boxsport im Vordergrund. Diese werden als Regeln am besten dadurch erlernt, indem die diesbezüglichen allgemeinen Gruppennormen ,,automatisch“ übernommen oder das vorbildliche Lehrer- oder Trainerverhalten nachgeahmt werden. Die grundlegenden Wettkampfregeln müssen mit dem Ziel, den eigenen Verhaltensspielraum voll auszuschöpfen, vermittelt werden. Die sich aus den Regeln ergebenden taktischen Möglichkeiten werden eher implizit gelernt und lassen sich sehr effektiv durch Kampfformen in Verbindung mit direkten Hervorhebungen schulen. Die elementaren taktischen Regeln werden am Besten als Leit- und Erfolgssätze erlernt. Solche Erfolgsregeln, die die allgemein effektivsten Lösungen für bestimmte Situationen vorgeben, sind sehr entscheidend für die spätere taktische Entwicklung der Nachwuchsboxer. Der Erwerb der wichtigsten strategischen und taktischen Leitsätze darf nicht dem Zufall überlassen werden, sondern muss gezielt durch theoretischen Taktikunterricht herbeigeführt werden. (vgl. LANGHOFF; SCHOTT, 1983; HOHMANN; LAMES, 2002) 


\subsection{Das Training der Taktik im Boxen}

Der antreibende Mechanismus, der der Informationsorganisation beim Erwerb von immer komplexeren und zugleich immer ausdifferenzierteren Wahrnehmungs- und Bewegungsmustern zu Grunde liegt, wird mit Hilfe des Prinzips der antizipativen Verhaltenskontrolle nach HOFFMANN (1993) erklärt. (vgl. ROTH, 1996) Danach tragen Lernen und Verhaltensentwicklung in hohem Maße aktive Züge: Psychische Prozesse und insbesondere die hierarchische Organisation der Neuronennetze werden nicht durch ermüdungsbedingte Auslenkung und Anpassung, sondern durch prospektive Erwartungen initiiert. Vor Handlungsbeginn gehen die subjektiven Erwartungen in antizipierte Handlungsergebnisse über. Treten die antizipierten Handlungsergebnisse bei der Handlungsrealisation ein, werden die Erwartungen gefestigt. Divergieren erwartete und eingetretene Handlungsergebnisse hingegen, differenzieren sich die Handlungserwartungen aus. Diese Ausdifferenzierung wird umso komplexer, je mehr antizipativ gesteuerte Teilprozesse in einem Handlungsprozess vermischt werden.

ROTH (1996) gehen davon aus, dass das taktische Verhalten in einfachen Handlungssituationen durchaus beiläufig und ungeplant, also durch sog. inzidentelles Lernen angeeignet werden kann. Dem gegenüber wird in komplexen Situationen ein angemessenes taktisches Verhalten erfolgreicher durch ein Zielgerichtetes und systematisch geplantes intentionales Lernen erreicht. Allerdings hat sich diese These in einschlägigen Untersuchungen bislang nur zum Teil bestätigen lassen. (vgl. ROTH, 1996; RAAB, 1999) Dies kann nach BARTH (2003) u. U. mit den verschiedenartigen Betrachtungsmodellen des strategischtaktischen Trainings in den Zweikampfsportarten erklärt werden.

Taktiklernen geschieht auf zweierlei Weise. Auf der einen Seite werden im Verlauf des Trainings immer wieder taktische Erfahrungen inzidentell, d. h. zeitlich eher beiläufig und inhaltlich eher zufällig gesammelt. Auf der anderen Seite werden im Training taktische Lernprozesse intentional geplant und taktische Kenntnisse, Fähigkeiten und Fertigkeiten systematisch vermittelt. Beim intentionalen Taktiktraining unterscheidet man Trainingsmethoden, bei denen die Ver- 
besserung des taktischen Verhaltens implizit, $d$. h. indirekt durch die häufige Wiederholung bestimmter Kampfsituationen angesteuert wird, von solchen Trainingsmethoden, bei denen das angestrebte taktische Verhalten direkt über explizite Instruktionen erreicht werden soll.

Dem Taktiktraining liegen im Allgemeinen drei Kategorien von Trainingszielen zu Grunde: taktische Kenntnisse, Fähigkeiten und Fertigkeiten (vgl. Abb. 5).

\begin{tabular}{|l|l|l|l|}
\hline \multicolumn{1}{|c|}{$\begin{array}{c}\text { Strategie } \\
\text { Strategische } \\
\text { Kenntnisse }\end{array}$} & \multicolumn{1}{|c|}{$\begin{array}{c}\text { Taktische } \\
\text { Kenntnisse }\end{array}$} & \multicolumn{1}{|c|}{$\begin{array}{c}\text { Taktische } \\
\text { Fähigkeiten }\end{array}$} & \multicolumn{1}{c|}{$\begin{array}{c}\text { Taktische } \\
\text { Fertigkeiten }\end{array}$} \\
\hline -Moralische & -Taktische & -Wahrnehmung & -Individuelle Fertigkei- \\
Regeln & Regeln & $\begin{array}{l}\text { - Antizipation } \\
\text {-Taktisches } \\
\text { Gedächtnis } \\
\text {-Strategische } \\
\text { Regeln }\end{array}$ & - Wettkampfre- \\
geln & $\begin{array}{l}\text { - Reaktionsschnellig- } \\
\text { keit }\end{array}$ & $\begin{array}{l}\text {-Teilizollektive Fertig- } \\
\text { keiten } \\
\text {-Kollektive Fertigkeiten }\end{array}$ \\
\hline
\end{tabular}

Abb. 5: Systematisierung der Boxtaktik (modifiziert nach: HOHMANN, 1996)

\subsection{Das Training taktischer Fähigkeiten im Boxen}

Die taktischen Fähigkeiten spiegeln sich insbesondere in der Qualität von Handlungsentscheidung und Handlungsausführung wider. Sie sind somit grundsätzlich über das Entscheidungshandeln in der Wettkampfsituation zu erschließen. Während hierüber im Kampf Einigkeit herrscht, bestehen große Unklarheiten in Bezug auf die Struktur der taktischen Fähigkeiten und den Grad ihrer Unabhängigkeit von einer konkreten Sportart. Aus diesem Grund kann man beim Training der taktischen Fähigkeiten derzeit lediglich auf die in der Abbildung 5 angeführte Zielkategorie zurückgreifen. Bei den taktischen Kenntnissen geht es um den kognitiven Erwerb. Taktische Fähigkeiten sind grundsätzlich als Talent vorhanden, müssen aber geschult werden. Taktische Fertigkeiten müssen im Detail geschult werden, da sie auf den Fähigkeiten aufbauen. (vgl. SCHNABEL; HARRE u. a., 1994) 


\subsection{Das Training taktischer Fertigkeiten im Boxen}

Im Unterschied zu den im Hintergrund wirkenden taktischen Fähigkeiten wird bei den taktischen Fertigkeiten von einem höheren Grad der praktischen „Verfestigung" ausgegangen. Die situationsbezogene Wahrnehmunge ist assoziativ eine bedürfnisartige Handlungstendenz, die eine bestimmte, individuell bevorzugte technische Fertigkeit auslösen soll. Daraus folgt, dass taktische Fertigkeiten sich in individuellen Verhaltensgewohnheiten manifestieren. (vgl. BARTH, 1995)

Die taktischen Fertigkeiten werden im Anfängerstadium als boxkampfspezifische Handlungen, wie z. B. das Fintieren, erlernt. Dabei ist es weniger schwierig, den technomotorischen Handlungsablauf korrekt auszuführen als zunächst eine günstige Anwendungssituation zu erkennen, in der die entsprechende Handlung erfolgreich eingesetzt werden kann. Für den Boxwettkampf sind die wichtigsten taktischen Fertigkeiten direkt im komplexen Wettkampf zu erlernen und dabei vereinfachte Techniken bei ggf. gleichzeitiger Vereinfachung der Kampfsituation zuzulassen. Für den Anfänger besteht das entscheidende taktische Trainingsziel darin, die handlungsauslösenden Signale gezielt zu suchen und sicher zu identifizieren. Damit die Nachwuchsboxer dies lernen, muss der Lehrer die Stellen, an denen die Schlüsselreize zu finden sind, explizit verdeutlichen. (vgl. LETZELTER, 1987; LIPPMANN; SPITZ u. a., 2000 )

\subsection{Trainingspraxis im Boxen}

CARL (1984) definiert Training als einen komplizierten Handlungsprozess mit dem Ziel des planmäßigen und sachorientierten Einflusses auf die boxsportliche Leistungsentwicklung. Als Komplex wird in diesem Begriff ein Handlungsprozess bezeichnet, der darauf ausgerichtet ist, angemessene Wirkungen bei allen Eigenschaften des Boxers zu erzielen, die leistungsbestimmend sind. Davon ausgeschlossen sind alle Maßnahmen, die sich aus ethischen Gründen verbieten. LETZELER (1987 ) definiert Training als ein Verfahren zur Optimierung und Stabilisierung des psycho-physischen Leistungszustandes, in dem Trainingsinhalte mit angemessen Trainingsmethoden, welche nach den Grundsätze des 
sportlichen Training angeordnet und auf vorgegebene Trainingsziele ausgerichtet sind, ausgeführt werden. Die Fähigkeit und Bewegungen mit hochgradiger Qualität auszuführen, sind von großer Bedeutung grade in dem Bezug auf die Techniken beim Fintieren im Boxen. Nicht nur in den technischkompositorischen Sportarten, die im Kern aus der Präsentation perfekter Bewegungen bestehen, sondern auch in allen anderen Sportartengruppen ist Technik sehr wichtig. (vgl. MARTIN, 1977; CARL, 1983; HOHMANN, 1994)

Für BARTH $(2003,26)$ ist der "Gegenstand von Strategie und Taktik... ein System optimaler Handlungs- und Verhaltenspläne zur Situationsangemessenen Realisierung sportlicher Aufgaben und Ziele im Wettkampf. Eingeschlossen sind das dazu erforderliche Wissen und Können. Das besondere am 'Strategen' oder 'Taktiker' ist, dass er sein Handeln und Verhalten selbst organisiert und es nach den Regeln der Effektivität, der Gewinnmaximierung und Verlustminderung plant und realisiert." BASTIAN (2003) verdeutlicht am Beispiel des Trainings in der Unterdruckkammer, wie hierbei Boxspezifisches Training mit einem (an Leichtathletik orientiertem) Ausdauertraining verbunden und eine hoch signifikante Leistungssteigerung erzielt wird. Das Training der individuellen Kampfhandlungen wurde hierbei vor allem durch gezielte Partnerwechsel, Vorgabe von Handlungsdichtwerten, ständige Fehlerkorrektur und Videosofortauswirtung geschult. 


\section{Technik und Taktik im Boxen}

\subsection{Forschung über die Technik und Taktik im Boxen}

PORSUGJAN; FARFEL (1978) haben eine Untersuchung ausgeführt, über die Dauer der Schlagausführung beim Boxen und ihre zeitliche Verkürzung. Der Ausgangspunkt für sie war eine Verkürzung der Schlagzeit beim Boxen. Hierzu wurde eine Lederpuppe aufgestellt, die mit Fäusten ausgestattet war. Durch einen eigens konstruierten Mechanismus wurden sowohl die Fäuste in Bewegung gesetzt als auch Signale gegeben, die der Versuchsperson anzeigen, auf welchen Teil des Körpers oder des Kopfes sie den Schlag zu richten hatte. Dabei wurde die Reaktionszeit mit einer Genauigkeit von bis zu 0,015 Sek. gemessen. Die 19 Versuchspersonen (darunter acht Spitzensportler) haben in je drei bzw. vier Tests 180240 Schläge ausgeführt. Am deutlichsten kommt der zeitliche Unterschied zwischen Kopf- und Körperschlägen zum Vorschein, und zwar 0,46 +/- 0,029 Sek für Körper- und 0,48 +/- 0,047 Sek für Kopfschläge. Zweitens ist festzustellen, dass die Schläge von Linksauslegern schneller als von Rechtsauslegern ausgeführt werden und drittens, dass gerade Schläge schneller sind als Schläge von der Seite oder von unten. Das Training der Schlaggeschwindigkeit und -dichte bei unterschiedlichen Belastungen fand auch in der DDR (BASTIAN, 2003) besondre Beachtung.

TOPYSCHJOW; DSHEROJAN (1979) haben eine Analyse der Schlagtechnik der besten Boxer ausgeführt. Dies zeigt individueller Eigenheiten auf, die jedem Boxer zu Eigen sind. Die Verfasser bestimmten die technischen und physikalischen Grundlagen des Boxschlags, die allgemeingültig sind. Drei Arten von Boxschlägen werden unterschieden. 1. Schläge mit minimaler Schnelligkeit und maximaler Kraft. Sie sollen die Deckung des Gegners öffnen. 2.Schläge mit optimaler Geschwindigkeit und Kraft als Hauptelement des Angriffs. 3. Schläge mit maximaler Geschwindigkeit und Kraft. Sie werden im Kampf episodisch in besonders günstigen, taktisch vorbereiteten Situationen angewendet. 
KULIEV (1980) hat eine Untersuchung ausgeführt, die in verschiedenen Experimenten die optimale Ausführung der Schlagtechnik im Boxkampf anstrebt. Die Sportgruppe, die drei Monate täglich zwei Stunden trainierte, bestand aus 20 Boxern der 2. und 3. Klasse. Die Untersuchung fand in einer Vorbereitungsperiode eines Wettkampfes statt. Der technisch-physische Zustand der Sportler wurde aufgenommen. Mit der Methode der Elektrodynamographie wurde die Zeit, die zwischen einem Doppelschlag lag, bestimmt. Man maß die Bewegungskoordination der einzelnen Körperteile und ihre zeitlichen Beziehungen. Die nach einer speziellen Methode ausgearbeiteten Übungsprogramme fanden im Verlauf von 5 Wochen statt, um die noch unvollkommene Technik beim Doppelschlag - verursacht durch Bewegungsfehler - zu korrigieren. Eindeutige Verbesserungen der Schlagtechnik (Verkürzung der Schlagzeit) und der Beinarbeit waren am Ende des Experiments zu verzeichnen. Die Ausarbeitung spezieller Übungen zur Trainingsgestaltung unterstützte die Entwicklung technischer Fähigkeiten im Nahkampf.

KULIEV (1981) zeigt in seiner Forschung, dass im Boxen vorrangig die Technik des Nahkampfes beherrscht werden muss. In einem Versuch wurde die Doppelschlagtechnik bei Boxern mit hochgradiger sportlicher Qualifikation untersucht. Es stellte sich heraus, dass die Doppelschlagserie nicht als Summe von zwei einzelnen isolierten Schlägen anzusehen ist, sondern als eine technische Einheit, die aus fünf relativ selbständigen Phasen besteht. In der Doppelschlagserie hat der erste Schlag die Funktion des Abschätzens und des Öffnens der gegnerischen Verteidigung, der zweite ist darauf gerichtet, den gezielten Schlag zu versetzen. Nach der Annäherung und der Ausführung des ersten Schlages erfolgt der Rückzug des Rumpfes in die optimale Position für den zweiten Schlag. Dabei ist nicht notwendigerweise die Distanz zu vergrößern. Für die Ausführung beider Schläge ist eine starke Kraft der Beine notwendig, um die maximale Geschwindigkeit der Schlagbewegung entfalten zu können.

KACURIN; KISESEV (1993) haben zur Entwicklung der Fähigkeit von Boxern zur genauen Bewertung der Hauptparameter der Bewegung und Effektivitätsbestimmung der spezielle motorische Vorbereitung eine Untersuchung mit jungen Boxern durchgeführt (25 Nachwuchsboxer der Leistungsklasse 2 und 3 im 
Alter von 18-21 Jahren). Die Athleten lernten ihre Schlagbewegungen richtig zu verteilen, dass sie sowohl leichte als auch starke und akzentuierte Schläge anbringen konnten. Die Wettkampfergebnisse und pädagogischen Beobachtungen sollen Beweise für die Effektivität der Anwendung der Methode der "Sofortinformation" und der speziellen Übungen zur Vervollkommnung der Zeit-, Schnelligkeits- und Kraftdifferenzierungen im Trainingsprozess sein.

\subsection{Die technischen Grundelemente des Boxens}

Die Technik des Boxens lässt sich auf verhältnismäßig wenige Grundelemente zurückführen, die jedoch entsprechend der konkreten Kampfsituation in ungleichen Varianten und Verbindungen beherrscht werden müssen und so den beträchtlichen Umfang des technischen Repertoires des Boxens ausmachen. Darüber hinaus ist es verbindlich, vom Beginn des Trainings an auch die wichtigsten Arten der Grundelemente zu lehren, um so gute technische Voraussetzungen für die situationsgebundene Anwendung zu schaffen. (vgl. BARISCH, 1953; GREBE, 1984; STAUFFER, 1993; SONNENBERG, 1993)

In dieser Arbeit werde ich die Finten analysieren, und in diesem Zusammenhang zeige ich die Beziehung zwischen dieser Fertigkeit (Boxstellung - Fortbewegung - gerade Schläge - Verteidigung) und den Finten auf.

Die Finten mit den Beinen (einfache Finten - kombinierte Finten) kann der Boxer schnell lernen, wenn er gute Flexibilität und Kondition in seiner Beinarbeit zeigt. Gerade Schläge sind die dominierende Schläge, bei den von mir analysierten Olympischen Spielen 2000. Für den Gegner ist dies aber auch die optimale Situation, er kann aufgrund der gegebenen Distanz variabler fintieren.

\subsection{Die Boxstellung (Kampfstellung)}

Die Kampfstellung ist die Position, von der aus der Boxer seine Taktik ausführen kann. Sie hilft ihm auch die Taktik des Gegners zu erkennen, so ist sie Ausgangstellung für Angriff, Verteidigung, Fintieren und Gegenangriff. Die Kampfstellung ist zugleich Ausgangs- und Endstellung aller Angriffs- und Verteidi- 
gungshandlungen und muss die Haltung des Oberkörpers, Kopfhaltung und Haltung von Armen und Fäusten den individuell optimalen Bedingungen entsprechen.

Vier Punkte sind bei der Kampfstellung von Bedeutung:

1. Während des Kampfes und in den Pausen sollte sich der Boxer nicht mental und damit auch physisch verkrampfen, er sollte also Lockerheit bewahren.

2. Gute Standfestigkeit und das Verlagern des Körpergewichtes auf beide Beine ist für das Gleichgewicht verantwortlich.

3. Die größtmögliche Gewandtheit in allen Körperachsen und Bewegungsrichtungen bedeutet leichte Bewegungen.

4. Durch spezifische Arm-, Bein- und Kopfhaltung sowie der Körperstellung gewährleistet die Deckung meist Schutz. (vgl. HIRSCHFELD, 1953; DENZ, 1997; FIEDLER, 1997)

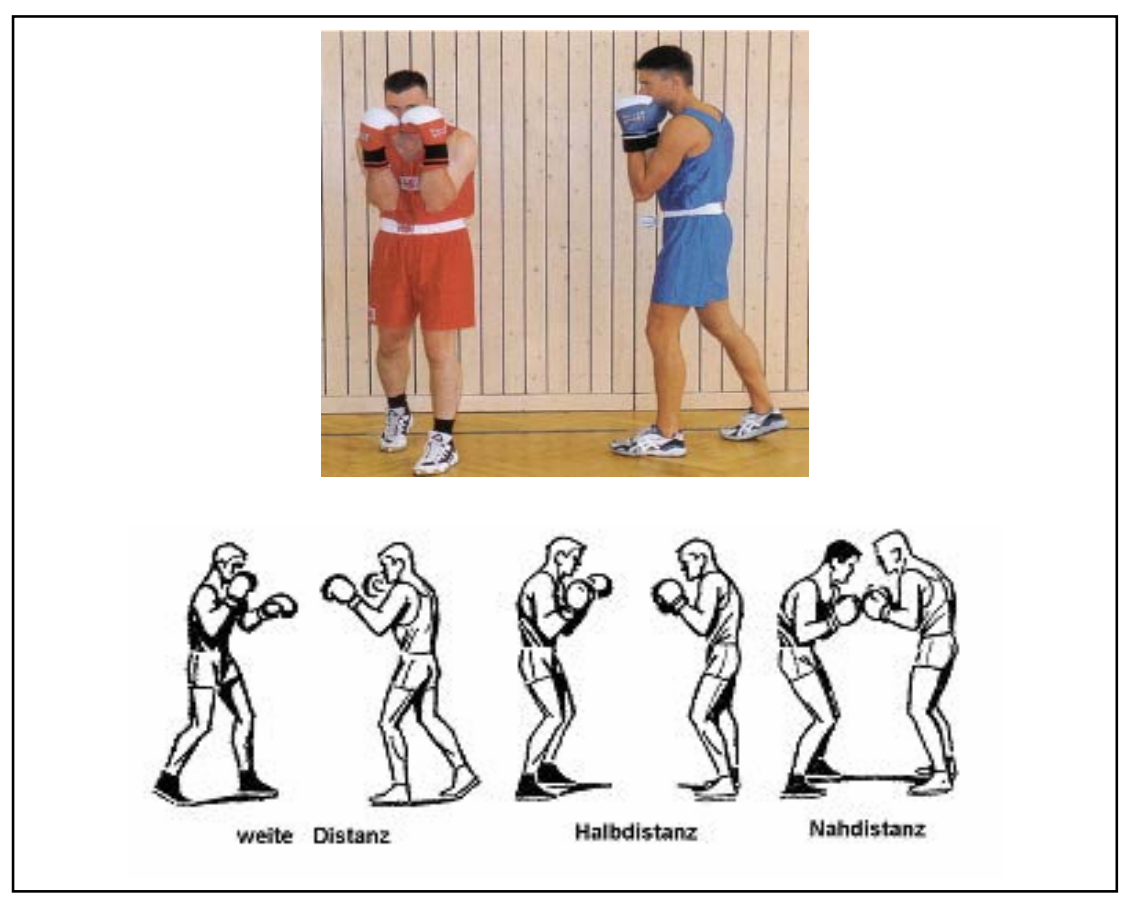

Abb. 6: Die Boxauslagen in verschiedenen Distanzen

(modifiziert nach: ELLWANGER; ELLWANGER, 1998; FIEDLER, 1997) 
Beim Boxen gibt es zwei verschieden Boxauslagen. Es gibt: Linksausleger und Rechtsausleger. Zur Begründung des Begriffs Auslage: Boxer, bei denen die linke Faust und der linke Fuß vorn sind, heißen in der Fachsprache Linksausleger. Bei Rechtsauslegern sind umgekehrt die rechte Faust und der rechte Fuß vorn. In der Regel ist bei Linksauslegern die rechte Hand die stärkere, also die Schlaghand. Bei Rechtsauslegern ist meist die linke Hand die Schlaghand. Äußerste Aufmerksamkeit ist bei den sogenannten "Verkappten" geboten. Sie weichen von der Regel ab. Die stärkere Hand ist vorn. (vgl. RÄSCH, 1973; OATES, 1988; MÜLLER, 1991; ELLWANGER; ELLWANGER, 1998)

\subsection{Die Fortbewegung im Boxen}

Die Fortbewegung dient dazu, dass der Boxer im Ring sich schnell und gleichmäßig bei Angriff, Verteidigung, Gegenangriff und Finten bewegen kann. Mit dieser Fortbewegung kann der Boxer auch die Schläge des Gegners vermeiden, keine Verletzung bekommen und danach kann er ggf. den Wettkampfsieg erreichen.

Es gibt Grundsätze zum gleichmäßigen Schutz der Boxer während ihre Bewegungen in verschiedene Richtungen gehen.

1. Für eine sichere Standtechnik ist es erforderlich die Füße angemessen der Körperbreite um einen Schritt auseinander zu platzieren.

2. Der Schwerpunkt muss auf beide Beine gleichmäßig verteilt sein.

3. Die Gliederung der Fußarbeit sollte wie in Abb. 7 aufgezeigt, nach Möglichkeit von dem Boxer befolgt werden.

Unter Fachleuten ist bekannt, dass Spitzenprofis verschiedener Sportarten, u. a. Fußball und Tennis nach ähnlichen Methoden wie die Boxer ihre Beinarbeit trainieren.

Grundsätzlich unterscheiden wir zwischen schreitend gleitender und federndgleitender Beinarbeit bzw. Fortbewegung des Boxers sowie in Verbindung mit den Boxschlägen zwischen Diagonal- und Passgang. (vgl. BARISCH, 1953; SONNENBERG, 1989) 
Gute Boxer beherrschen alle Fortbewegungsarten (nach vorne, nach hinten, nach rechts, nach links, Zirkelbewegung nach rechts, Zirkelbewegung nach links).

Wenn der Einsteiger diese Fortbewegung gut gelernt hat, kann er ganz einfach die Finten mit den Beinen leisten, zum Beispiel die einfachen Finten (Halber Vorwärtsschritt, Halber Rückwärtsschritt) und die kombinierten Finten.

1. Die Beine mit dem Rumpf (Halber Schritt nach vorne mit: a. Vorwärts ausweichen, b. Vorwärts ausweichen links, c. Vorwärts ausweichen rechts)

2. Die Beine mit dem Armen:

a. Halber Schritt nach vorne mit: (1. Kurzer gerader Schlag, 2. Schwacher Schlag, 3. Bewegung Schulter nach vorn)

b. Halber Schritt zurück mit: (1. Kurzer gerader Schlag, 2. Schwacher Schlag, 3. Bewegung Schulter nach vorn)

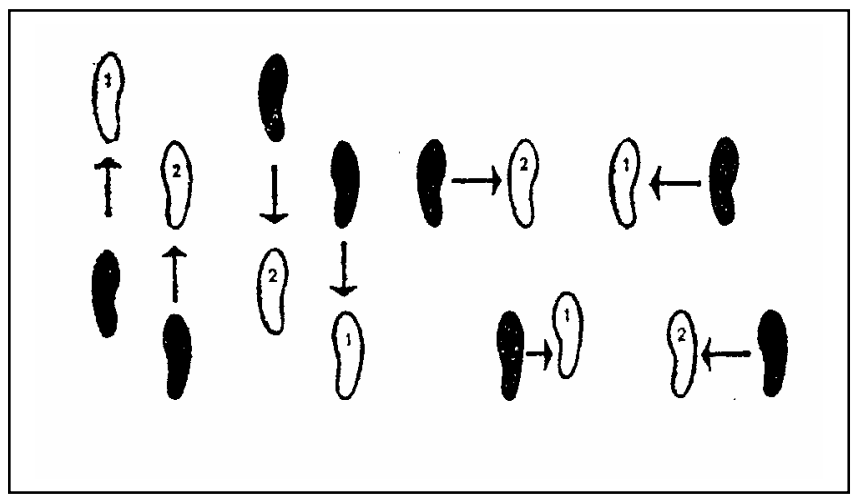

Abb. 7: Fortbewegung schreitend-gleitend nach vorn, hinten, rechts und links (nach: FIEDLER, 1997, 28)

\subsection{Die Grundschläge im Boxen}

Mit den Grundschlägen im Boxen erreicht der Boxer mit zwölf verschiedenen Grundschlagarten die Mittel zu einem erfolgreichen Abschluss eines Kampfes. Folgende Grundschläge sind aufgeführt :

1. gerade Schläge mit der Führungshand zum Kopf, 
2. gerade Schläge mit der Führungshand zum Oberkörper,

3. gerade Schläge mit der Schlaghand zum Kopf,

4. gerade Schläge mit der Schlaghand zum Oberkörper,

5. Seitwärtshaken mit der Führungshand zum Kopf,

6. Seitwärtshaken mit der Führungshand zum Oberkörper,

7. Seitwärtshaken mit der Schlaghand zum Kopf,

8. Seitwärtshaken mit der Schlaghand zum Oberkörper,

9. Aufwärtshaken mit der Führungshand zum Kopf,

10. Aufwärtshaken mit der Führungshand zum Oberkörper,

11. Aufwärtshaken mit der Schlaghand zum Kopf.

12. Aufwärtshaken mit der Schlaghand zum Oberkörper

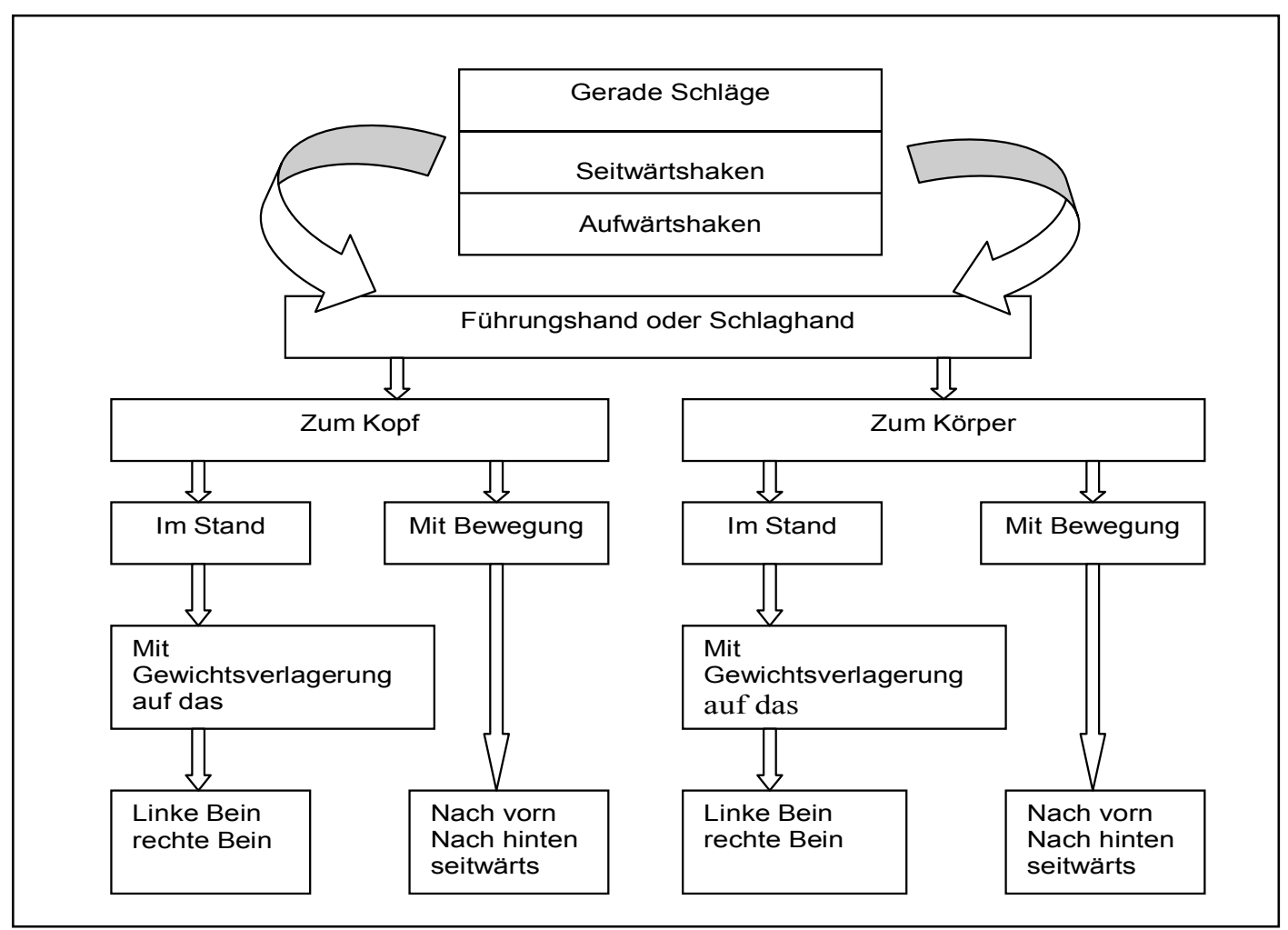

Abb. 8: Die Boxschläge (modifiziert nach: FIEDLER, 1976)

Zuerst werden die Boxschläge dem Boxer im Verlaufe seiner technischen Übung im Stand und in Verbindung mit Schritten in allen Bewegungsrichtungen gelehrt. In der Praxis werden alle die Schläge in Verbindung mit den Treffermöglichkeiten benannt (Kinntreffer, Halstreffer seitlich, Halstreffer frontal, Herztreffer, Solarplexustreffer, Rippentreffer, Bauchtreffer). Dies sind die so genann- 
ten „K.-O.-Punkte”. Es wird deshalb in der Boxliteratur von sieben Trefferstellungen gesprochen. (Vgl. FIEDLER, 1974; SONNENBERG, 1978; OATES, 1988)

Durch meine Erfahrung im Boxsport bin zu dem Resultat gekommen, dass die so genannten sieben Trefferstellen um fünf Stellen zu erweitern sind.

Im derzeitigen Boxsport sollten diese zusätzlichen Trefferbereiche dem Boxer während seiner Ausbildung vermittelt werden, um im späteren Kampf die optimalen Vorteile der erhöhten Trefferflächen nutzen zu können.

Die sich damit ergebenen Möglichkeiten beziehen sich natürlich auch auf das Fintieren. Hier ist der Boxer mit den neuen Schlagpunkten vertraut zu machen, um den Gegner weiterführend irritieren zu können.

Die nachfolgende Skizze zeigt die gesamten 12 Trefferbereiche auf. Von den in der Skizze aufgeführten Trefferbereichen sind die Bereiche 1, 2, 3, 4 und 7 von mir zusätzlich zu den üblichen Trefferbereichen aufgenommen worden.

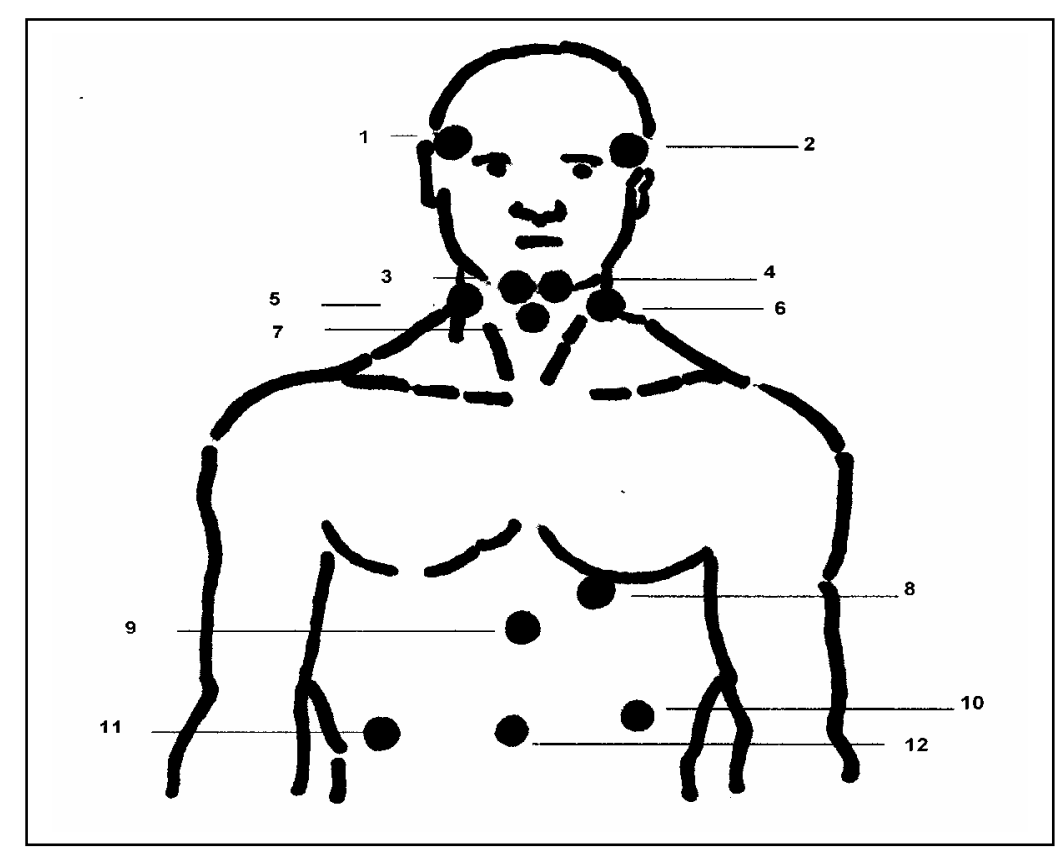

Abb. 9: Die Schlagempfindlichen Körperstellen (K. - O.-Punkte) des Boxers (eigene Darstellung)

Nachfolgend werden weitere Schlagarten aufgezeigt, die in der Ausübung sowohl als Angriff als auch der Verteidigung und dem Fintieren dienen. 
1. Die Vorbereitungsschläge: Das Ziel dieser Schläge ist die Öffnung der Deckung und eine Finte für dem Gegner, um weitere Grundlagenschläge platzieren zu können. Diese Art von Schlägen ist wichtig in Bezug darauf, dass der Gegner nur einen Scheinangriff erfährt und durch die Finte des Angreifers verunsichert ist. Bei einfachen Finten mit den Armen (kurzer gerader Schlag) und bei Kombinierten Finten mit Beinen und Armen macht der Boxer einen halben Schritt nach vorne mit kurzem, geradem und schwachem Schlag oder er macht einen halben Schritt zurück mit dem selben Schlagverhalten

2. Die Grundlageschläge: Die Grundlageschläge folgen meistens mit hoher Intensität nach den Vorbereitungsschlägen des Angreifers. Je nach Intensität des Schlages kann ein Punkt erreicht werden.

3. Die Gegenschläge: Sind die Schläge zur Verteidigung der Grundschläge. Sie erfolgen, indem der sich zu verteidigende Kämpfer je nach Schlagart kontert.

\subsection{Die geraden Schläge}

In dieser Arbeit werde ich nur auf gerade Schläge eingehen, weil sie die wichtigsten Schläge im modernen Boxen sind, desgleichen sind sie die dominierenden Schläge bei Weltmeisterschaften und Olympischen Spielen. Es gibt außerdem einen gegenseitigen Zusammenhang zwischen Finten und geraden Schlägen, weil der Boxer nach einfachen oder kombinierten Finten schnell gerade Schläge aus der weiten Distanz machen kann, aber für die anderen Schläge (Seitwärtshaken, Aufwärtshaken, etc.) braucht der Boxer eine Fortbewegung nach vorne mit den Beinen, um sich über eine kurze oder nahe Distanz fortzubewegen; erst dann kann er die Seitwärtshaken oder Aufwärtshaken schlagen. Somit sind die geraden Schläge die logischen Schläge im Zusammenhang mit Finten. Wie oben gesehen, sind sie zudem die schnelleren Schläge. 
1) Die geraden Schläge mit der Führungshand zum Kopf: Wendet der Boxer häufig an, weil es leichte und schnelle Schläge sind. Die Anwendung im Angriff aus weiter Distanz und die Anwendung im Manöver und die Finten sind auch wichtige Schläge, um viele Punkte zu bekommen.

1.1) Die Gerade mit der Führungshand zum Kopf aus dem Stand: Bei den meisten Menschen ist es die linke Hand, die ungeschickter ist. Sie wird meistens weniger eingesetzt, somit ist in der Regel die linke Hand als die Führungshand zu bezeichnen. Im früheren Boxsport diente sie nur der Vorbereitung für den Einsatz der kräftigeren Hand, der so genannten Schlaghand. Die Führungshand soll den Einsatz der Schlaghand vorbereiten, kann aber auch selbst enorme Kraft entwickeln. Der Schlag der Führungshand ist keine Armstreckung, sondern eine Ganzkörperbewegung. Verbunden mit dem Strecken des linken Arms ist der Zehenballenabdruck des rechten Beins, der eine Gewichtsverlagerung auf den linken Fuß einleitet. Linke Beckenseite und linke Schulter werden gleichzeitig mit der Armstreckung nach vorn gebracht. Die linke Schulter berührt dabei die linke Seite des Kinns. Wird der Kopf nicht in diese Gesamtbewegung einbezogen, entwickeln sich Fehler, die später nur noch schwer zu korrigieren sind. Wird der Schlag mit einem Schritt ausgeführt, verlagert sich das Gewicht ruckartig auf den vorderen Fuß, der kurz vor dem Auftreffen beim Stoß der linken Faust fest stehen muss. Unter extremen Bedingungen werden so die Kraft des hinteren Beines, die Rumpfkraft und die Armkraft in die gesamte Stoßkraft eingebracht. Dabei gilt aber, dass die Schrittgröße die Schnelligkeit des Schlages bestimmt: Je größer der Schritt, desto langsamer die Gesamtbewegung und somit der Schlag. (vgl. SONNENBERG, 1978; WEISE, 1995; ELLWANGER; ELLWANGER, 1998) 


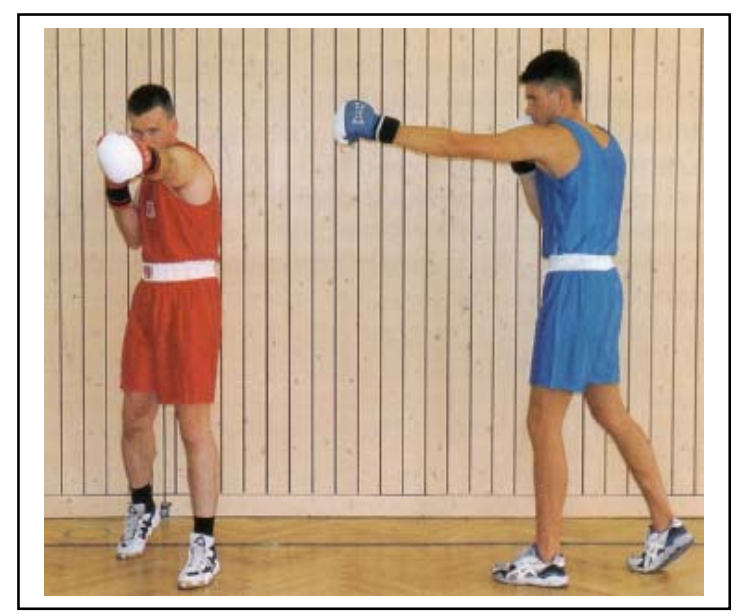

Abb. 10: Die linke Gerade zum Kopf aus dem Stand von vorn und von der Seite (nach: ELLWANGER; ELLWANGER, 1998, 52)

1.2) Die Gerade mit der Führungshand zum Kopf in Bewegung: Die Boxer, die die Gerade aus der Bewegung mit der Führungshand schlagen wollen, müssen nachfolgende Bewegungen absolvieren. Durch Abdruck mit dem hinteren Fuß wird der vordere Fuß gleichzeitig mit der Führhand etwa 5 bis $20 \mathrm{~cm}$ vorwärtsbewegt. Dabei wird der vordere Fuß nach vorn geschoben (schreitendgleitend) und mit dem Auftreffen des Schlages das Körpergewicht auf die Fußsohle des sich streckenden Beines verlagert. Beim Auftreffen der Faust muss die Vorwärtsbewegung fertig sein.

Nachsetzen des hinteren Fußes und das Zurückziehen der Faust in die Ausgangsposition erfolgen gleichzeitig. Bei geraden Führhänden in Verbindung mit anderen Fortbewegungsrichtungen verhält es sich ähnlich, nur dass der Schlag in Verbindung mit Schrittbewegungen nach links, rechts oder rückwärts ausgeführt wird. (vgl. SONNENBERG, 1989; FIEDLER, 1997)

Die oben unter 1.2) genannte Fertigkeit hilft bei der Instruktion von kombinierten Finten mit den Beinen und Armen (Halber Schritt nach vorne mit kurzem geraden Schlag, schwacher Schlag, halber Schritt zurück mit kurzem geraden Schlag, schwacher Schlag) 


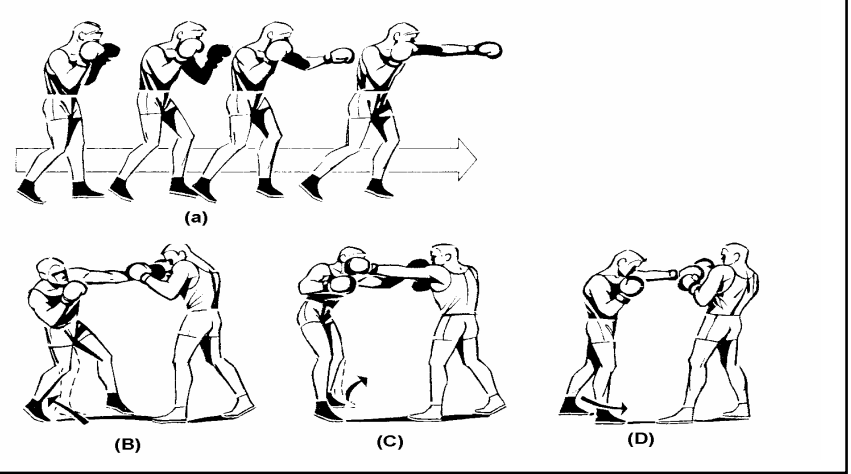

Abb. 11: Die linke Gerade zum Kopf mit einem Schritt nach vorn (A), nach hinten (B), nach links (C) und nach rechts (D)

(nach: FIEDLER, 1997, 36)

2) Die geraden Schläge mit der Führungshand zum Oberkörper: Diese Art der Schläge werden nach meiner Studie und meiner Erfahrung weniger angewandt als die Schläge zu 1).

2.1) Die Gerade mit der Führungshand zum Oberkörper aus dem Stand: Dieser Schlag entspricht im Wesentlichen der Geraden mit der Führungshand zum Kopf. Der Unterschied ist hier die Kniebewegung. Man muss den Schlag mit leichtem Beugen im Knie ausführen.

Diese Fertigkeit hilft bei der Instruktion der einfachen Finten mit den Beinen (Abtauchen) und diese Fertigkeit ist wichtig nach diesen Finten, um einen geraden Schlag zum Oberkörper zu stoßen.

Der oben angeführte Schlag kann sowohl mit der Führungs- und auch der Schlaghand ausgeführt werden. (vgl. FIEDLER, 1997) 


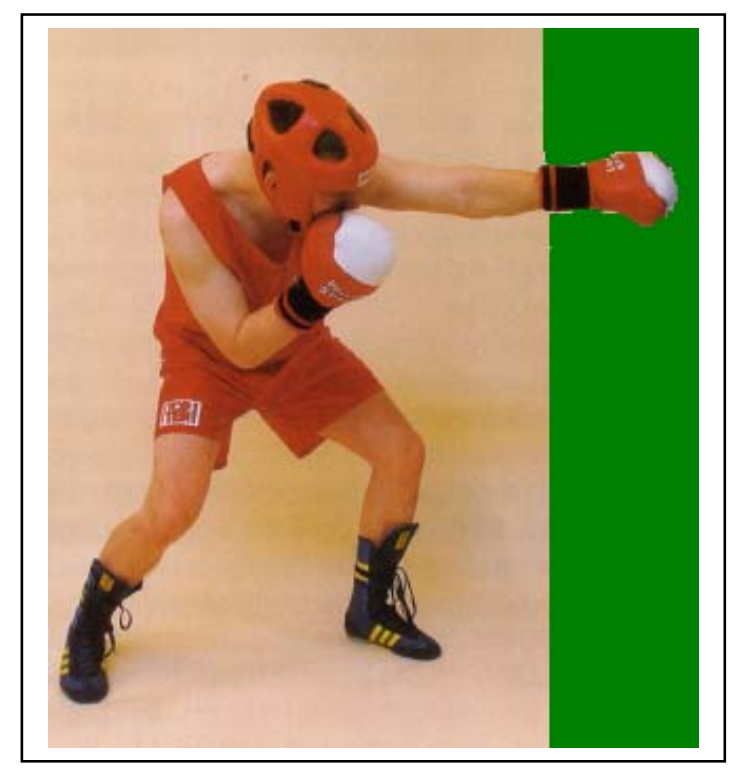

\section{Abb. 12: Die linke Gerade zum Oberkörper aus dem Stand}

(eigene Darstellung)

2.2) Die Gerade mit der Führungshand zum Oberkörper in Bewegung: Der Bewegungsablauf entspricht im Grundsatz dem der Geraden zum Kopf. Der Unterschied besteht darin, dass der Oberkörper stärker nach vorn geneigt ist und seitlich in Richtung der Schlaghand während der Schlagausführung. GröBere Sportler verbinden die Neigung des Oberkörpers mit leichtem Beugen im Knie des vorderen Beines. Die Deckungshand muss konsequent Kopf und Körper sichern. (vgl. SONNENBERG, 1989)

Diese Schläge helfen bei der Anwendung der einfachen Finten mit den Beinen (Abtauchen) und bei der Anwendung der kombinierten Finten mit den Beinen und Armen. Nach dieser Finte kann der Boxer einen geraden Schlag zum Oberkörper ausführen. 


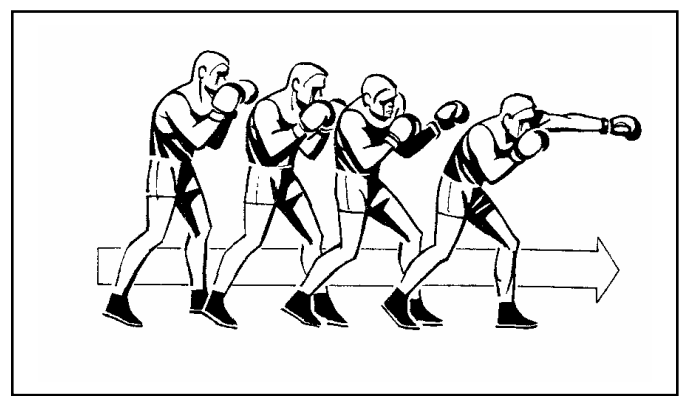

Abb. 13: Die Gerade mit der Führungshand zum Oberkörper in Bewegung (nach: FIEDLER, 1997, 38)

3) Die geraden Schläge mit der Schlaghand zum Kopf: Die Schlaghand hat neben ihrer Funktion als Deckungshand bei Schlägen mit der Führungshand vor allem die für den Kampfverlauf entscheidende Aufgabe, Schlagwirkungen zu erzielen. Dabei spielt die richtige taktische Vorbereitung durch Manöver und Finten, das kontrollierte Herausarbeiten günstiger Kampfpositionen und die richtige Ausführung des Schlages eine entscheidende Rolle.

3.1) Die Gerade mit der Schlaghand zum Kopf aus dem Stand: Dieser Schlag ist einer der stärksten Schläge, die der Boxer nach dem Vorbereitungsschlägen anwenden kann. Besonders nach vorhergehendem Einsatz von Finten, kann der Boxer einen Punkt oder sogar einen K- O- Sieg bekommen, da dies ein sehr wirksamer Schlag ist.

Zur Ausführung des Schlages kann der Boxer aus der Boxstellung den Schlag schnurgerade ohne Auftaktbewegung vom Kinn ins Ziel schlagen. Die Bewegung fängt mit dem Abdruck des hinteren Beines an und wird durch das nach vorn bringen der Hüfte und Schulter des Schlagarmes gestützt. Das Körpergewicht wird auf das vordere Bein verlagert, das hintere Bein nach dem Abdruck fast gestreckt und die Ferse, ohne mit dem Fußballen den Bodenkontakt zu verlieren, leicht angehoben. Während des Schlages wird die Faust der Führungshand zur Deckung leicht geöffnet an das Kinn zurückgeführt. Nach dem Schlag wird sofort wieder die Boxstellung eingenommen. (vgl. FIEDLER, 1974; STAUFFER, 1993; ELLWANGER; ELLWANGER, 1998) 


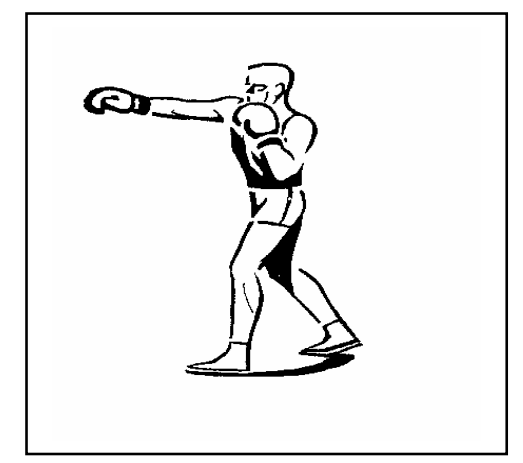

Abb. 14: Die Gerade mit der Schlaghand zum Kopf im Stand

(nach: FIEDLER, 1997, 40)

3.2) Die Gerade mit der Schlaghand zum Kopf in Bewegung: Der Boxer kann diesen Schlag lernen, nachdem er die Gerade mit der Schlaghand im Stand geübt hat, dann wird in der Vorwärtsbewegung der vordere Fuß und die Schlaghand gleichzeitig nach vorn geführt. Beim Auftreffen des Schlages hat der Fuß seine Vorwärtsbewegung beendet und steht fest auf dem Boden. Mit dem Nachstellen des hinteren Fußes wird die Faust auf kürzestem Wege in die Ausgangsstellung zurückgeführt. (vgl. SONNENBERG, 1978; FIEDLER, 1997)

Diesen Schlag kann der Boxer wie eine Finte benutzen, z. B. mit einfachen Finten mit dem Armen (Kurzer gerader Schlag) und mit Kombinierten Finten mit Beinen und den Armen:

A. Mit halben Schritt nach vorne mit kurzem geraden schwachen Schlag.

B. Mit halben Schritt zurück mit kurzen geraden schwachen Schlag. 


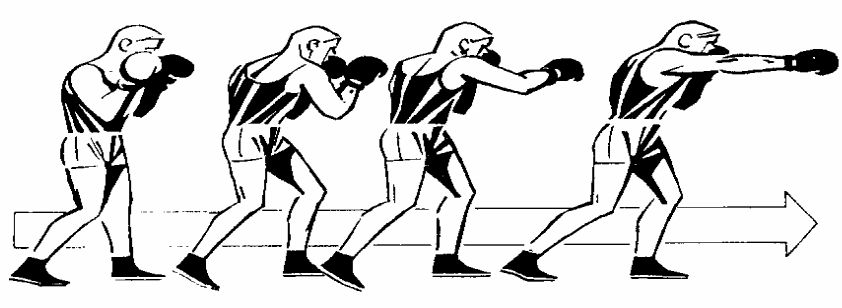

Abb. 15: Die Gerade mit der Schlaghand zum Kopf in Bewegung

(nach: FIEDLER, 1997, 40)

4) Die geraden Schläge mit der Schlaghand zum Oberkörper: Diese Art der Schläge werden nach meiner Studie und meiner Erfahrung weniger angewandt als die Schläge zu 3).

4.1) Die Gerade mit der Schlaghand zum Oberkörper aus dem Stand: Dieser Schlag ist wie die rechte Gerade zu dem Kopf. Der Unterschied liegt in der Kniebewegung, der Boxer beugt seine Knie leicht (Abtauchen).

Dieser Schlag kann nach erfolgreicher Finte mit den Beinen (nur Abtauchen) angewandt werden. Ebenso nimmt er eine wichtige Rolle nach kombinierten Finten mit den Armen und Beinen ein, um einen Treffer auf den Oberkörper zu platzieren. Das Erlernen dieses geraden Schlags eignet sich gut als Vorbereitung auf das Erlernen der erwähnten Finten. 


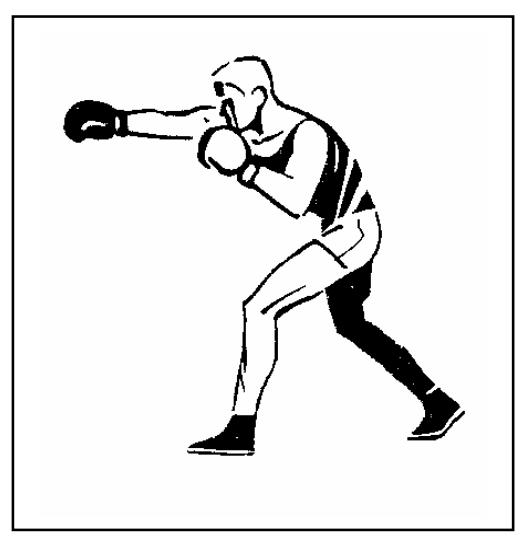

Abb. 16: Die Gerade mit der Schlaghand zum Oberkörper aus dem Stand (eigene Darstellung)

4.2) Die Gerade mit der Schlaghand zum Oberkörper in Bewegung: Der Bewegungsablauf entspricht im Grundsatz dem der Geraden zum Kopf. Der Unterschied besteht darin, dass der Oberkörper stärker nach vorn geneigt ist und sich seitlich in Richtung der Führungshand während der Schlagausführung befindet. (vgl. SONNENBERG, 1993)

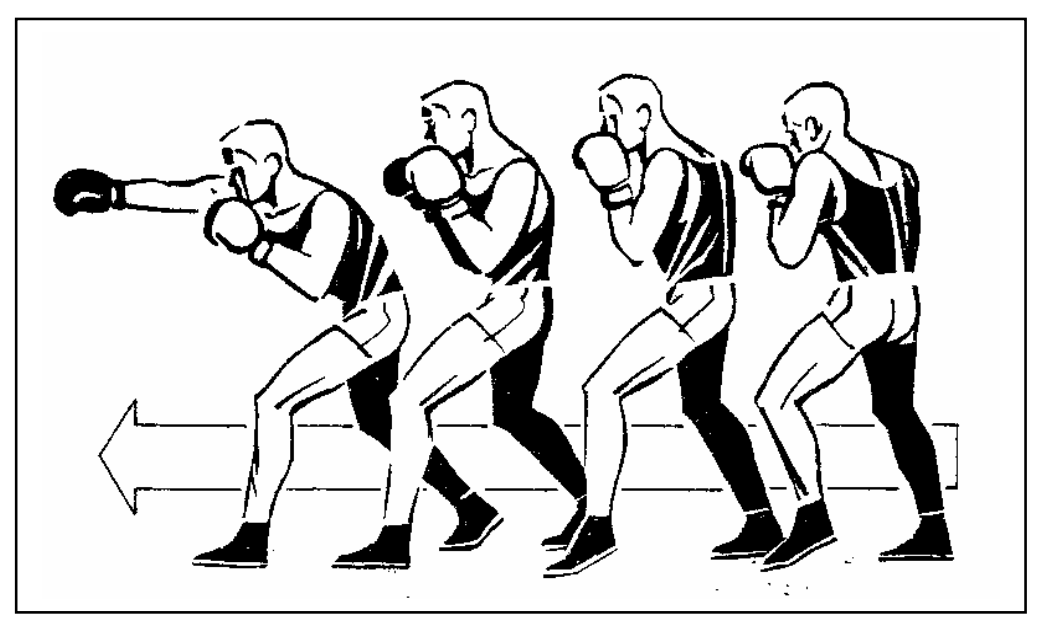

Abb. 17: Die Gerade mit der Schlaghand zum Oberkörper in Bewegung ( nach: FIEDLER, 1997, 41) 


\subsection{Die Schlagfolgen}

Ein Merkmal des modernen Boxens ist die Fähigkeit zur Verbindung von Schlägen in einem harmonischen Bild mit leichter und schneller Fortbewegung von einer Position zur anderen, um den Boxer die leichte und schnelle Auswahl der taktischen Mittel zu ermöglichen. Die Boxerfähigkeit zeigt sich in der Verbindung von Schlägen in Schlagfolgen.

Vor dem Erlernen der Schlagfolgen sollte der Boxer jedoch mit Manövern und Finten anfangen. Sie sind die Vorbereitung auf Schläge, die eine Bresche bei dem Gegner öffnen. Diese ausgewählten Schlagfolgen (Schlagverbindung, Schlagkombination, Schlagserien) sind abhängig vom freien Platz des Gegners, und der Boxer muss diese Schlagfolgen häufig trainieren, bis er ein ausreichendes Niveau der Beherrschung bekommt.

Unter Schlagfolgen ist die direkte Aufeinanderfolge von mindestens zwei Grundschlägen zu verstehen. Sie können im Angriff, in der Verteidigung und im Gegenangriff angewendet werden. Grundsätzlich unterteilen wir die Schlagfolgen in Schlagverbindungen, Schlagkombinationen und Schlagserien. (vgl. OGURENKO, 1972; FIEDLER, 1997)

Die technische Vielfalt ergibt sich zum großen Teil aus Verbindungsmöglichkeiten der unterschiedlichen Finten und den passenden Grundschläge zu einer harmonischen Gesamtbewegung. Die fließenden Übergänge von Auftakt- bis zur Endbewegung der aufeinander folgenden Schläge, die Koordination von Körper-, Arm- und Beinbewegungen und auch die ständige Gewichtsverlagerung stellen hochgradige Anforderungen an das Niveau der speziellen koordinativen Fähigkeiten des Boxers. (vgl. SONNENBERG, 1978; FIEDLER, 1976) 
3.7.1 Die Schlagverbindung: Diese Art von Schlagfolgen ist die Verbindung gleichartiger Grundschläge. Dabei überwiegen in den ersten Jahren der Boxausbildung die Verbindungen gerader Stöße in der weiten Distanz. Im Folgenden einige Beispiele von Schlagverbindungen. Eins-Zwei-Schlag (Gerade mit der Führungshand und Gerade mit der Schlaghand zum Kopf).

Die Ausführung der Bewegung entspricht den bereits beschriebenen Einzelschlägen. Beide Schläge werden jedoch direkt nacheinander ausgeführt, so dass in der Vorwärtsbewegung (zu Beginn der Ausbildung im Passgang) beim Vorsetzen des linken Beines die linke Gerade (Führungshand) und mit dem Nachsetzen des rechten Beines die rechte Gerade (Schlaghand) geschlagen wird. Dieser Grundsatz - jeder Schlag mit einem Schritt verbunden - hat durchgängig für alle Schlagfolgen Gültigkeit. Dabei können die Folgen jeweils im Pass- oder Diagonalgang und nach allen Bewegungsrichtungen ausgeführt werden. (vgl. OGURENKO, 1972; SONNENBERG, 1989)

So kann z. B. auch die Zweierverbindung entsprechend der konkreten Kampfsituation mit der Schlaghand begonnen werden und die Führungshand zur Absicherung des Angriffes (schnelles Einnehmen der Kampfposition) folgen. Diese Verbindung erfolgt im Diagonalgang, die Rechte (Schlaghand) ist überfallartig und kraftvoll mit einem Vorwärtsschritt des linken Beines verbunden. In der Abb. (18) ist die gebräuchlichste Variante der Verbindung zweier gerader Stöße dargestellt.

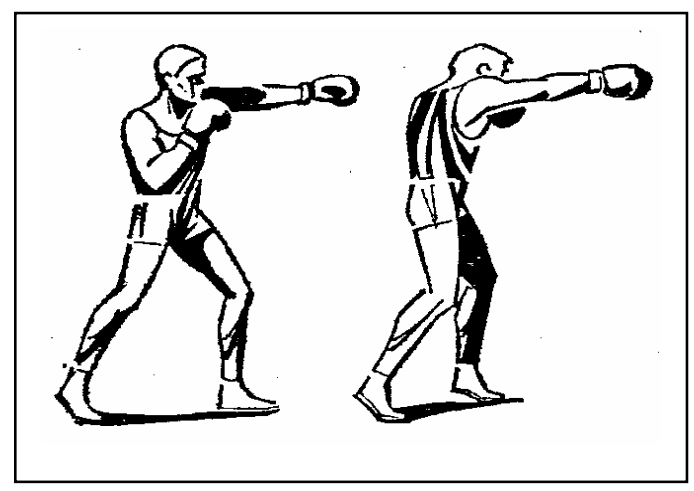

Abb. 18: Zweierschlagverbindung gerader Stoß zum Kopf (nach: FIEDLER, 1997, 44) 
Der Dreierschlag (Gerade mit Führungshand, Schlaghand, Führungshand). Diese Verbindung wird häufig im Kampf angewendet. Sie wird der Forderung einer Boxausbildung gerecht, Schlagfolgen nach Möglichkeit mit der Führungshand einzuleiten (Fintieren, Vorbereitung des Wirkungsschlages) und zu beenden (Herstellen der Distanz, Stören des Gegenangriffs, Einnehmen der Kampfposition). Die Dreier- Verbindung wird durch die unterschiedliche Akzentuierung der Schläge und durch die Variation von Kopf- und Körperschlägen ein wirkungsvolles technisches Mittel, das auch von Spitzenkönnern häufig verwendet wird. (vgl. FIEDLER, 1976; ELLWANGER; ELLWANGER, 1998)

In der Abb. (19) ist die gebräuchlichste Variante der Verbindung dreier gerader Stöße dargestellt. Auf der linken Seite findet man die Darstellung einer Schlagverbindung. Gerade zum Kopf in der Bewegung (Führungshand, Schlaghand, Führungshand). Die rechte Seite der Abbildung zeigt die Verbindung zweimal Führungshand, Gerade zum Kopf mit folgender geraden Schlaghand zum Körper.

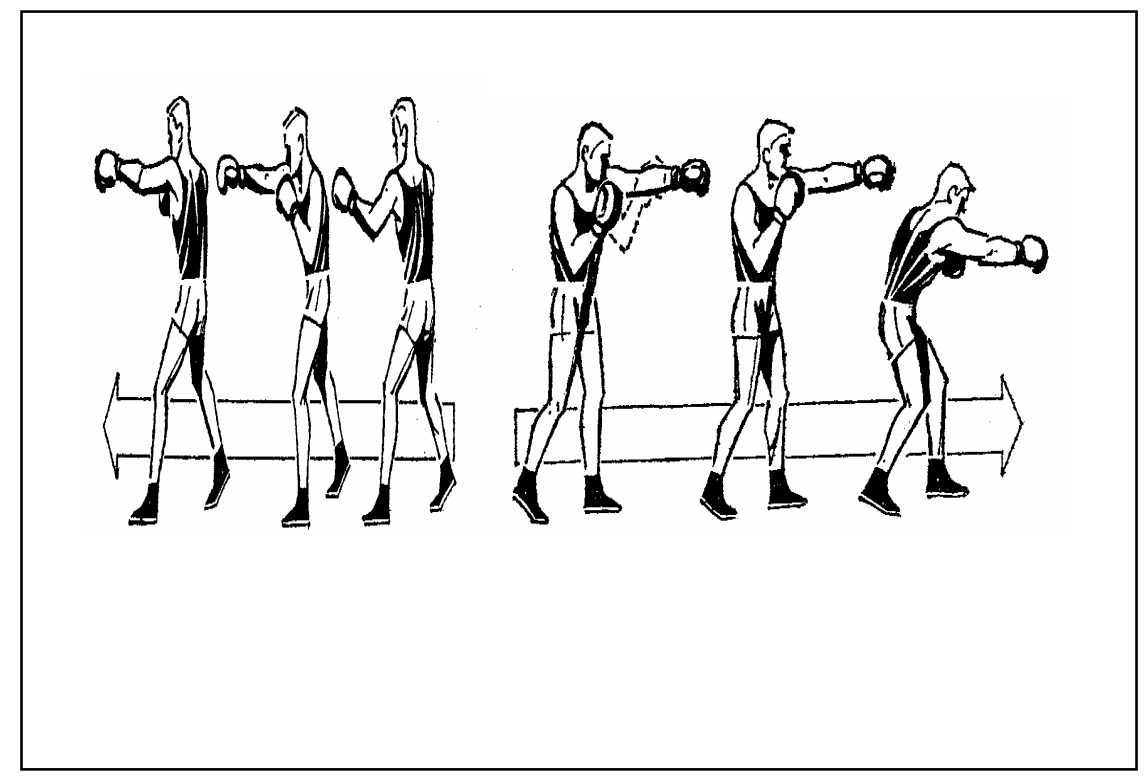

Abb. 19: Schlagverbindung (Dreierschlag)

(modifiziert nach: FIEDLER, 1997) 
3.7.2 Die Schlagkombinationen: Bei dieser Art von Schlagfolgen werden mindestens zwei verschiedenartige Grundschläge miteinander verbunden. Sie sollen in der Regel nicht mehr als fünf Schläge umfassen. Schlagkombinationen werden vorwiegend in der Halbdistanz angewendet. Im Prozess der dauerhaften Ausbildung sollte der Boxer befähigt werden, zumindest bewegungsmäßig alle Grundschläge im Zusammenhang mit den unterschiedlichen Bewegungsrichtungen und Gangarten (Pass- und Diagonalgang) zu verbinden. (OGURENKO, 1972; FIEDLER, 1997)

Der Boxer kann diese Schlagkombination in allen Distanzen (Weitdistanz, Halbdistanz und Nahdistanz) leisten. Er kann sie auch nach einer Finte (einfache oder kombinierte) leisten. Mit Rücksicht auf die Boxdistanz gibt es mehrere Bedingungen, die der Boxer berücksichtigen muss:

1. die Harmonie und der Rhythmus zwischen den Schlägen;

2. die Endposition für einen Schlag muss die Vorbereitung für den nächsten Schlag sein;

3. das Schlagziel sollte variabel gestaltet werden.

Der Boxer muss Rücksicht auf die folgenden Punkte nehmen:

1. Er soll während des Kampfes die Deckung nie vernachlässigen.

2. Regulierung der Distanz mit der Fortbewegung, besonders in Verbindung verschiedener Schläge.

3. Die Schlagkombination muss immer mit einem Vorbereitungsschlag anfangen.

4. Die Abstufung der Schlagkombination soll vom einfachen bis zum komplizierten Schlagen reichen.

Dabei steht vorerst die Absicht im Mittelpunkt der Ausbildung, durch kontinuierlich steigende Ansprüche an die motorische Lernfähigkeit und die speziellen koordinativen Fähigkeiten günstige Voraussetzungen für eine spätere Spezialisierung des Boxers auf bestimmte Schlagvarianten und Kombinationen zu schaffen. (SONNENBERG, 1989) 
In jeder einzelnen Schlagkombination wird der letzte, die Schlagkombination abschließende Schlag betont, z. B. in einer Schlagkombination aus drei Schlägen. Folglich, müssen die ersten beiden den Gegner von der Verteidigung jener Körperstellen ablenken, auf die der dritte Schlag gerichtet werden soll. Und beim zweiten Schlag muss der Boxer bereits bemüht sein, eine günstige Ausgangsstellung für den betonten dritten Schlag vorzubereiten. Eine systematische Vervollkommnung der Schlagkombination kann dem Boxer im richtigen Augenblick des Kampfes dazu verhelfen, eine bestimmte Schlagkombination fast automatisch, doch mit Betonung des letzten Schlages, anzubringen. Ein schneller und fließender Wechsel der Schläge in der Schlagkombination auf die verschiedenen verwundbaren Punkte des Gegners mit unterschiedlichen Richtungen und Techniken erschwert dem Gegner die Verteidigung, demoralisiert inn und zwingt inn in die Defensive. (vgl. OGURENKO, 1972)

In den folgenden Abbildungen sind zwei der gebräuchlichsten Kombinationen dargestellt.
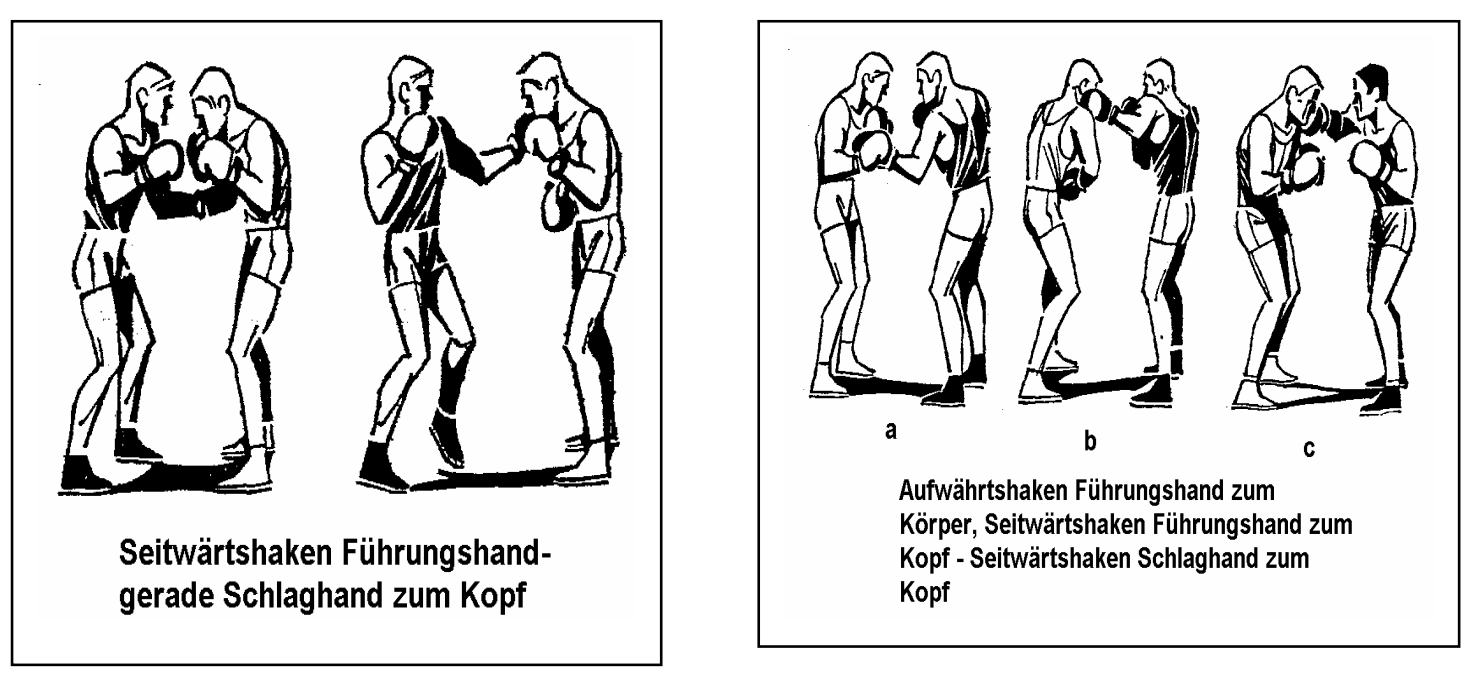

Abb. 20: Schlagkombination: der erste von zwei Schlägen und der zweite
von drei Schlägen (eigene Darstellung) 
Die Schlagkombinationen aus vier Schlägen bestehen entweder aus zwei Kombinationen von zwei Schlägen, zwei wiederholten Schlägen einer Hand und einer Kombination aus drei gleichförmigen Schlägen mit einem betonten, andersartigen vierten Schlag. (vgl. OGURENKO, 1972)

Die Schlagkombination in den Viererschlägen unterscheidet sich nicht nur in ihrer Form, sondern auch in der Kraft und Bewegungskoordination. Ein, zwei oder drei Schläge in der Schlagkombination sind ablenkender Art, der vierte ist der Hauptschlag. Beim ablenkenden (relativ schwachen) Schlag der Schlagkombination bereitet der Boxer seine Ausgangsstellung für den folgenden betonten Schlag vor. (SONNENBERG, 1978)

3.7.3 Die Schlagserien: Diese Art von den Schlagfolgen wird in der Regel in Nahdistanz angewendet. Die bestehen aus einer Vielzahl gleichartige Schläge. Mit einer Anzahl von Aufwärtshaken oder Seitwärtshaken wird beispielsweise im Nahkampf versucht, die Deckung des Gegners aufzureißen, um auf die Innenbahn zu kommen oder den Gegner zu veranlassen, seine Deckungsarbeit auf die Abwehr der vielen Aufwärtshaken oder Seitwärtshaken zu konzentrieren. Dadurch besteht die Möglichkeit, überraschend mit einem Kopfhaken auf Halbdistanz zu gehen. Boxer, die in Doppeldeckung oder auch am Seil stehen, werden mit einer Serie kurzer gerader Stöße oder auch Seitwärtshaken eingedeckt. (vgl. OGURENKO, 1972; FIEDLER, 1976; ELLWANGER; ELLWANGER, 1998)

Wählt man Schlagserien aus, müssen sie passend zu den Fähigkeiten der Boxer sein. Mit andauerndem Training kann der Boxer die Erfahrung erlangen, seine Schlagserien so auszuwählen, dass es ihm erlaubt in jeder Kampfsituation ein Maximum an Vorteilen zu nutzen.

In der folgenden Abbildung ist eine Schlagserie aus sechs Schlägen dargestellt: rechter Aufwärtshaken zum Körper, linker und rechter Haken zum Kopf, linker Aufwärtshaken zum Körper, rechte Haken zum Kopf, linker Haken zum Kopf. 


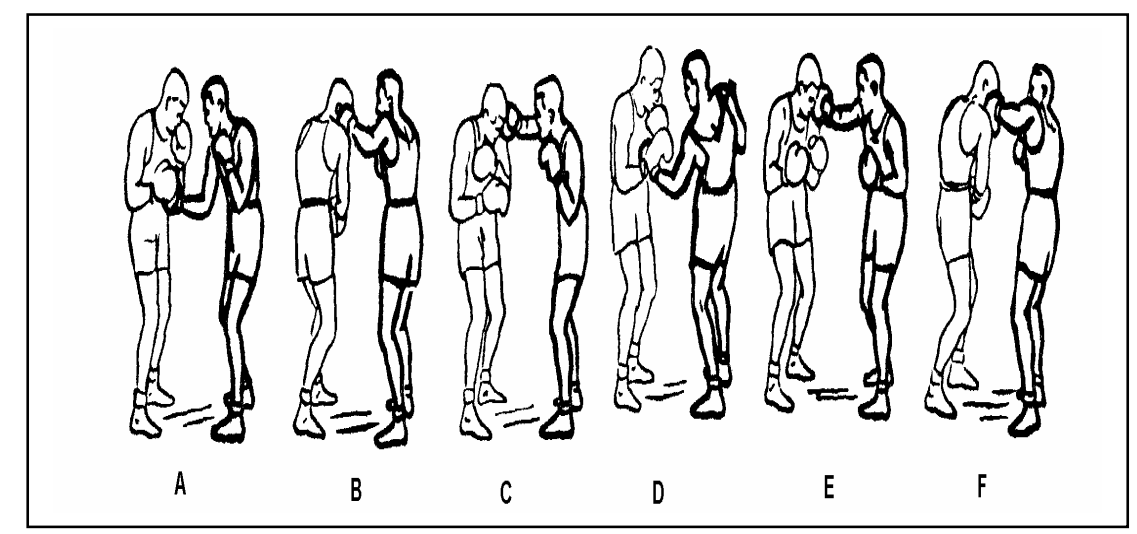

Abb. 21: Eine Schlagserie aus sechs Schlägen (eigene Darstellung)

\subsection{Die Verteidigung im Boxen}

\subsubsection{Die Verteidigungswichtigkeit des Boxen}

Die Verteidigung im Boxen ist die Bewegung einzelner oder mehrerer Körperteile (Arme - Rumpf - Beine), die der Boxer in der richtigen Zeit ausführt, um gegnerische Attacken abzuwehren.

Wichtig bei der Verteidigung ist:

1. Ein wichtiger Grundsatz ist es, die Gegenschläge zu vermeiden, um Verletzung zu vermeiden.

2. Der Boxer muss selbstbewusst seien, wenn die Verteidigung perfekt und gründlich ausgeführt werden soll.

3. Nach erfolgter Verteidigung sollte unbedingt der Gegenangriff eingeleitet werden.

4. Der Gegner kann seine Angriffstaktik nicht wie geplant ausführen, wenn der Boxer die Verteidigung perfekt ausführt.

\subsubsection{Die erfolgreichen Verteidigungsgrundsätze}

1. Der Schutz der Boxstellung:

Der Boxer muss seine Boxstellung schützen, um die Gegenschläge zu vermeiden. Der Gegner sollte keiner Bresche für Treffer finden. Der Boxer sollte die Lockerheit, Gleichmäßigkeit und Leichtbewegung dazu nutzen, seine Deckung immer optimal zu gestalten. 
2. Die Verteidigungsgeschwindigkeit :

Die Verteidigung muss augenblicklich ausgeführt werden, um dem Gegner keine langwierigen Attacken zu ermöglichen.

3. Zeit und Genauigkeit :

Die Verteidigung muss mit der passenden Zeit und Schnelligkeit die Abwehr perfekt ausführen.

4. Auswahl der Verteidigungsmittel :

Der Boxer muss die Verteidigung entsprechend seiner Fähigkeit und der Angriffstechnik des Gegners auswählen.

\subsubsection{Die Verteidigungsarten}

Es gibt zwei Arten der Verteidigung:

1. Passive Verteidigung: Bei der passiven Verteidigung führt der Boxer nur die Verteidigung ohne den Angriff aus.

Unter dem Begriff der passiven Verteidigung fallen alle Verteidigungshandlungen, die vom Boxer lediglich mit dem Ziel angewendet werden, Treffer des Gegners zu verhindern. Die Schläge werden mit den Schultern, den Armen, den geöffneten und geschlossenen Händen abgefangen oder abgelenkt (Deckungen, Paraden) oder man lässt sie durch Meid- und Ausweichbewegungen ins Leere gehen. Durch die Anwendung dieser Elemente verbucht der Boxer seinerseits keinen entscheidenden Vorteil. Er überlässt, beschränkt er sich auf die Anwendung der passiven Verteidigungselemente, die Initiative dem Gegner. Seine Kampfführung ist defensiv. Dennoch ist die gewissenhafte Ausbildung der passiven Verteidigungselemente von entscheidender Bedeutung. Ihre perfekte Beherrschung ist Grundvoraussetzung für die so genannte aktive Verteidigung. (vgl. FIEDLER, 1976; SONNENBERG, 1993)

2. Aktive Verteidigung: Bei der aktiven Verteidigung führt der Boxer die Verteidigung mit Gegenangriff aus. 
Zum Nachschlagen kommt es bei der aktiven Verteidigung. Es stellt eine Verbindung eines technischen Verteidigungselementes (passive Verteidigungshandlung) und einer Angriffshandlung dar. Das Nachschlagen ist die einfachste Form der aktiven Verteidigung und kommt im Verlaufe des Wettkampfes recht häufig vor. Der Boxer wehrt den gegnerischen Angriff ab und schließt unmittelbar daran Schläge an, um den Gegner an einer eventuellen AngriffsWeiterführung zu hindern bzw. seinerseits zum Treffer zu kommen. Das Nachschlagen kann in Verbindung zu allen beschriebenen Verteidigungselementen und in allen drei Distanzen erfolgen. Die Einheit zwischen Verteidigungs- und Angriffshandlungen kommt besonders gut in der Halbdistanz zum Ausdruck, wo beispielsweise beim Meiden und Nachschlagen der Ausklang der Verteidigungshandlung der Auftakt für die Ausführung des Schlages (Seitwärtshaken) ist. (vgl. FIEDLER, 1976; SONNENBERG, 1993)

Die effektivste Form der aktiven Verteidigung ist das Gegenschlagen. So wird der gegnerische Angriff bereits im Ansatz verhindert und der Gegner aus seinem Bewegungsrhythmus gebracht. Der erfahrene Boxer, der dieses aktive Verteidigungselement beherrscht, wird jederzeit kampfbestimmend sein. Die Fähigkeiten, sich im Schlagbereich des Gegners (Kontaktzone) sicher bewegen zu können, gegnerische Bewegungen im Ansatz zu erkennen oder sogar mit hoher Wahrscheinlichkeit zu prognostizieren sowie schnell und richtig zu reagieren, sind Voraussetzungen für eine solche Kampfweise. (vgl. OGURENKO, 1972)

Ich werde hier die Verteidigungsarten auch noch anders einteilen, nämlich in

\section{Einfache Verteidigung:}

Bei der einfachen Verteidigung verwendet der Boxer nur einen Körperteil (die Beine, den Rumpf oder die Arme), um die Verteidigung zu leisten.

\section{Kombinierte Verteidigung:}

Bei der kombinierten Verteidigung verwendet der Boxer zwei oder mehr Körperteile (Beine, Rumpf und die Arme), um die Verteidigung zu leisten. 


\subsubsection{Die Verteidigungsmethode}

1. Die Deckungen: Die Verteidigungselemente der Deckungen bestehen darin, dass der Boxer die gegnerischen Schläge mit der Faust, mit der geöffneten Hand, der Schulter, dem Unterarm oder dem Ellbogen abfängt. Die gewöhnlichsten Deckungen sind:

a) Block an der Schulter: Dieser Verteidigung ist einfach und leicht zu benutzen und wird gegen die Gerade mit der Schlaghand zum Kopf eingesetzt. Dabei wird durch blitzschnelles Verschieben der Schulter der gegnerische Schlag abgefangen. Gleichzeitig dreht sich der Boxer leicht nach rechts oder nach links je nach Angriffstypus (Links- oder Rechtshänder). Diese Drehung wird in der Kampfführung meistens als Auftakt für einen Antwortschlag benutzt. (vgl. SONNENBERG, 1978; FIEDLER, 1997)

Um einen ankommenden Schlag aufzufangen, wird die linke oder rechte Schulter so weit nach vorn gebracht, dass sie das Kinn und einen Teil des Gesichts abdeckt. Dabei entsteht wieder die verdeckte Ausholbewegung für die Schlaghand, da die jeweilige Schulter weit nach hinten verlagert wird. Ein solcher Block kann allerdings erst gelehrt werden, wenn der Boxer in der Lage ist, den kommenden Schlag zu beobachten. (vgl. ELLWANGER; ELLWANGER, 1998)

Wenn der Boxer diese Verteidigung ausführen kann, dann kann er auch die kombinierten Finten mit den Beinen und den Armen (einen halben Schritt nach vorn oder einen halben Schritt zurück, mit Bewegung der Schulter nach vorn) ausführen.

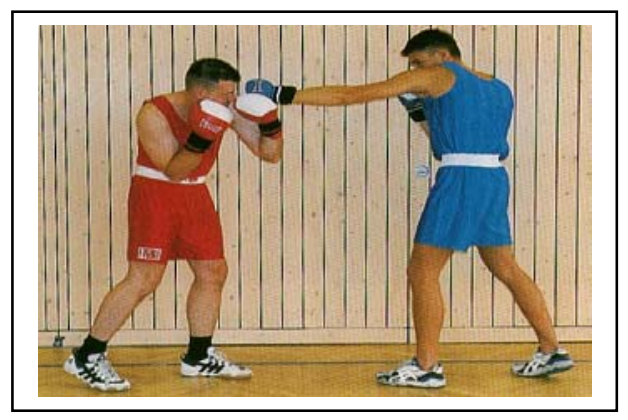

Abb. 22: Der Schulterblock (nach: ELLWANGER; ELLWANGER, 1998, 65) 
b) Kopfseitendeckung links und rechts: Diese Verteidigung wird gegen Seitwärtshaken zum Kopf (Kinnwinkel) eingesetzt. Der Boxer deckt sich, indem er die rechte bzw. die linke Faust an die Kopfseite führt. Die Faust bleibt geschlossen, und zur Vermeidung der Wucht der gegnerischen Schläge wird der Oberkörper leicht vorseitwärts aus der Schlagrichtung bewegt. Seitwärtshaken mit der Schlaghand werden mit der linken Faust (Kopfseitendeckung links) abgefangen und linke Seitwärtshaken mit der rechten Faust (Kopfseitendeckung rechts) abgefangen. (FIEDLER, 1997)

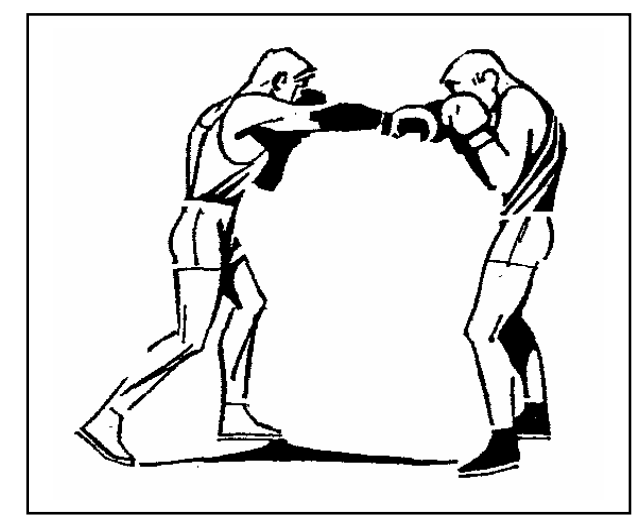

Abb. 23: Kopfseitdeckung links (eigene Darstellung)

c) Ellbogenblock links und rechts: Diese Verteidigung ist eine häufige Verteidigungsform. Der Boxer ist während seiner eigenen Angriffsaktion bestrebt, seine Körperpartie mit Ellbogen und Unterarm vor Schlägen zu schützen. Der Ellbogenblock wird durch eine Körperdrehung nach links ausgerichtet (gegen Schläge mit der rechten Hand) bzw. nach rechts (bei Schlägen mit der linken Hand) und wehrt so die Schläge mit dem Ellbogen ab. Bei Haken ist die Körperdrehung nur gering. Mit dem Ellbogenblock kann sich der Boxer gegen gerade Stöße, Seit- und Aufwärtshaken zum Körper verteidigen. (vgl. SONNENBERG, 1989; ELLWANGER; ELLWANGER, 1998)

Bei dieser Verteidigung sind die Körperbewegungen mit dem Rumpf, in diesem Fall einfache Finten (Vorwärtsausweichen links, Vorwärtsausweichen rechts) erforderlich. Wenn der Boxer diese Verteidigungshandlungen gründlich ausführt, kann er auch die kombinierten Finten mit den Beinen und dem Rumpf mit 
einem halber Schritt nach vorne (Vorwärtsausweichen links, Vorwärtsausweichen rechts) ausüben.

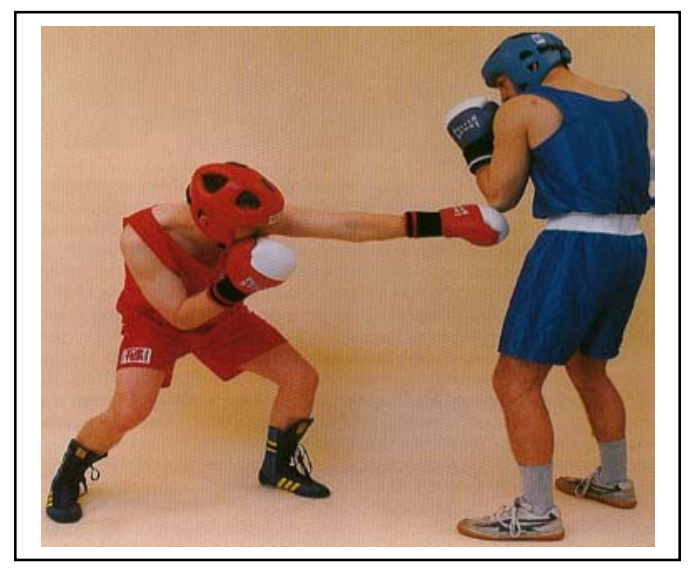

Abb. 24: Ellbogenblock links (nach: ELLWANGER; ELLWANGER, 1998, 94)

d) Doppeldeckung: Der Lernprozess der Doppeldeckung spielt jedoch im Kampfgeschehen nur im taktischen Verhalten eine Rolle. Bei der Doppeldeckung werden die Ellbogen solid an den Körper gepresst. Der Rücken wird rund gemacht. Das Kinn ist angezogen. Die Hände werden geöffnet und mit den Fingerspitzen an den Haaransatz gepresst. Das Handgelenk wird leicht nach außen gepresst. Also entsteht an den Handschuhen eine Auflagefläche und in der Mitte eine Spannung, die jeden Schlag aufnimmt und ihn nicht auf Körper oder Kopf überträgt. Die Knie werden ein wenig gebeugt. Wird die Führungshand geschlagen, lässt sich der Schlag auf diese Weise gut auffangen. Gleichzeitig sollte bereits zu diesem Augenblick darauf hingewiesen werden, dass diese Stellung auch taktische Vorteile bietet: Sie ermöglicht verdeckte Ausholbewegungen mit der Führungs- und auch der Schlaghand. (vgl. ELLWANGER; ELLWANGER, 1998)

Der Boxer fängt die geraden Stöße des Gegners durch Öffnen der vor dem Kinn gehaltenen Faust ab. Die Schlaghand (Kopfdeckung rechts) wird dabei am häufigsten eingesetzt. Schlägt der Boxer mit der Schlaghand, übernimmt die Führungshand (Kopfdeckung links) die Deckungsarbeit. Wird die vor dem Kinn geöffnete Hand etwas nach unten abgewinkelt, dann schützt die Kopfdeckung 
den Boxer vor gegnerischen Aufwärtshaken. (SONNENBERG, 1989; FIEDLER, 1997)

Bei der vorliegenden Verteidigung zeigt sich der Boxer mit beiden Armen bzw. Fäusten. Er versucht sich durch das Vorhalten beider Fäuste und der Unterarme, Kopf und Oberkörper vor wuchtigen Angriffen (viele Schläge) in Seilnähe zu schützen. Die Doppeldeckung ist äußerst unzweckmäßig, weil der Boxer dabei die Initiative abgibt und sie dem Gegner überlässt. (vgl. STAUFFER, 1993)

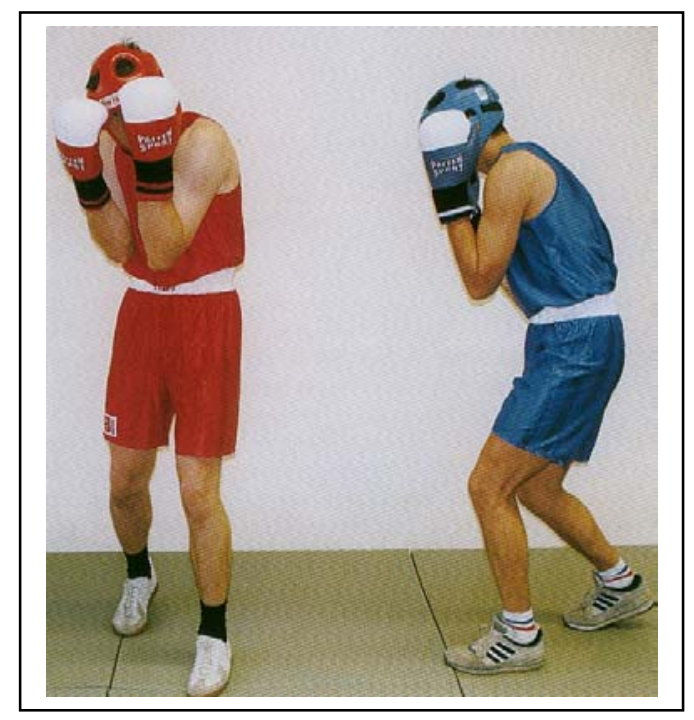

Abb. 25: Die Doppeldeckung (nach: ELLWANGER; ELLWANGER, 1998, 77)

2. Die Paraden: Diese Verteidigung benutzt man gegen die geraden Stöße mit der geöffneten Hand oder des Unterarmes. Sie können mit der Schlag- oder Führungshand ausgeführt werden. Dabei sollte in der Regel darauf geachtet werden, dass der gegnerische Stoß mit dem inm gegenüberliegenden Arm zu parieren ist, um möglichst wenige Treffmöglichkeiten zu bieten. (vgl. OGURENKO, 1972 )

In den folgen den Abbildungen sind dargestellt einige die Paraden

1. Parade mit der Schlaghand nach innen, 2. Parade mit der Führungshand nach innen, 3. Parade nach außen, 4. Parade nach oben. 


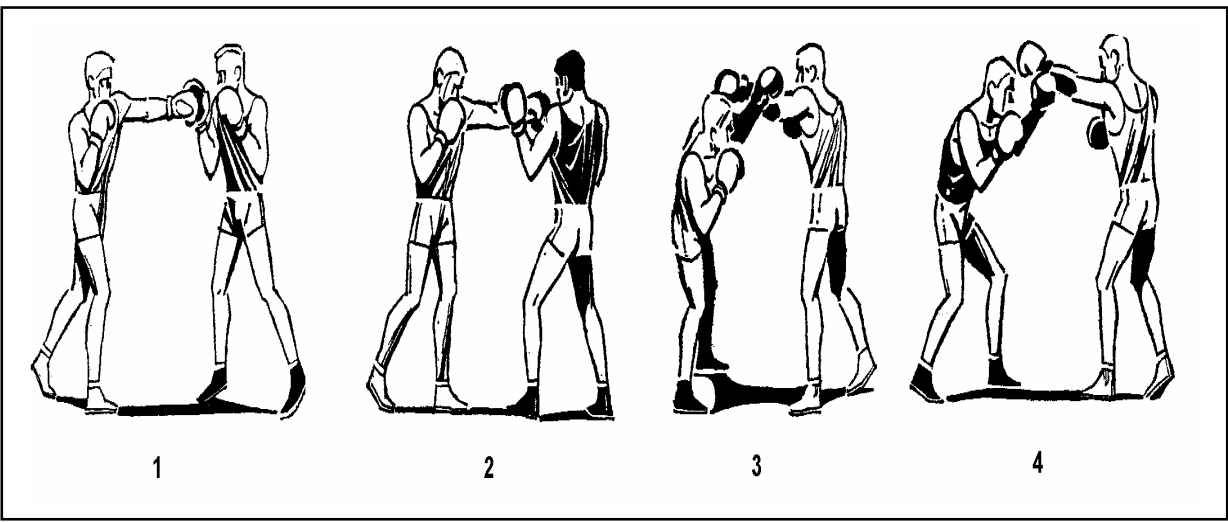

Abb. 26: Die Paraden (eigene Darstellung)

3. Die Meidbewegungen: Diese Verteidigung ist durch Bewegungen des Oberkörpers, die die Schläge der Gegner ins Leere gehen lässt, gekennzeichnet Diese Art der Verteidigung setzt ein gutes Beobachtungsvermögen und Reaktionsschnelligkeit voraus und findet vorwiegend in der Halbdistanz Anwendung. Zu den Meidbewegungen zählen wir hauptsächlich das Ducken, Rückneigen, Rollen und Pendeln.

Abducken schräg vorwärts: Diese Verteidigung findet meist gegen gerade Stöße zum Kopf Anwendung. Beim Erkennen des gegnerischen Schlagansatzes wird der Körper nach schräg vorn abgebeugt und deshalb aus der Schlaglinie des Gegners gebracht.

Die Verlagerung des Körpergewichtes auf das Bein der Beugeseite mit den leicht gebeugten Knies verlangt beim Vorbeugen besondere Aufmerksamkeit. Es ist darauf zu achten, dass der Kopf hinter den Fäusten bleibt und der Gegner nicht aus dem Blickfeld verloren wird. Gegen die linke Gerade zum Kopf wird nach rechts, gegen die rechte Gerade nach links abgeduckt. (vgl. OGURENKO, 1972; FIEDLER, 1997 ) 


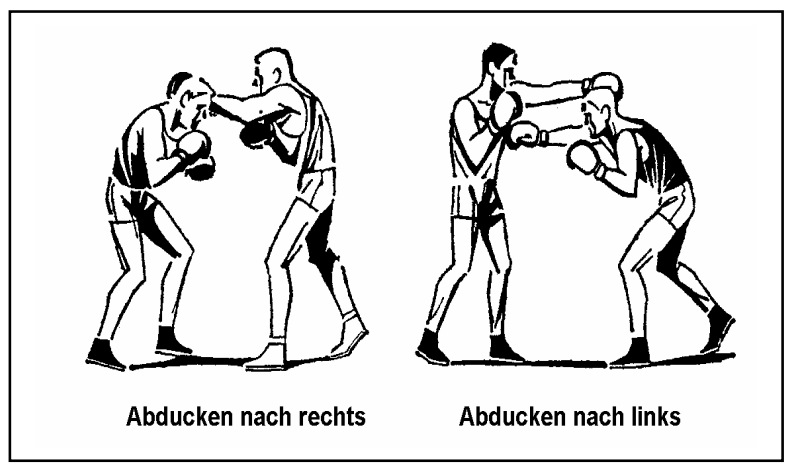

Abb. 27: Abducken nach rechts und nach links mit einer rechter Geraden zu Oberkörper (nach: FIEDLER, 1997, 64)

Dies ist eine Verteidigung, bei der mit einfachen Finten mit dem Rumpf (Vorwärts ausweichen, Vorwärts ausweichen links und Vorwärts ausweichen rechts) gearbeitet wird. Auch in den kombinierten Finten mit den Beinen und dem Rumpf mit halber Schritt nach vorn (Vorwärts ausweichen, Vorwärts ausweichen links und Vorwärts ausweichen rechts) wird dementsprechend gearbeitet.

Abducken nach unten (knicksen): Um Treffer mit der Führungshand am Kopf zu verhindern, muss der Kopf bis auf die Brusthöhe gebracht werden. Hierbei gibt es unterschiedliche Techniken. Aus der Boxstellung heraus wird der linke Fuß $20 \mathrm{~cm}$ nach vorn bewegt oder der rechte Fuß etwa $20 \mathrm{~cm}$ nach hinten; gleichzeitig werden beide Knie gebeugt. Eine andere Methode ist es, beide Knie in der Zeit der Beugung kurz zusammenzudrücken, die Boxstellung aber beizubehalten - diese Bewegung ist schneller und weniger kraftintensiv. Erfolgt zu diesem Zeitpunkt ein Vorbringen der rechten oder linken Schulter, ist ein kraftvoller Stoß mit Gewichtsverlagerung möglich. (vgl. ELLWANGER; ELLWANGER, 1998)

Gegen gerade Stöße oder Seitwärtshaken zum Kopf findet insbesondere in Halbdistanzen "das Abducken nach unten" Anwendung. Der Boxer macht schnell eine leichte Kniebeuge und neigt den Oberkörper nur wenig nach vorn. Es bietet sich für die Weiterführung des Kampfes aus dieser Position das Schlagen von Aufwärtshaken an. (vgl. SONNENBERG, 1993) 


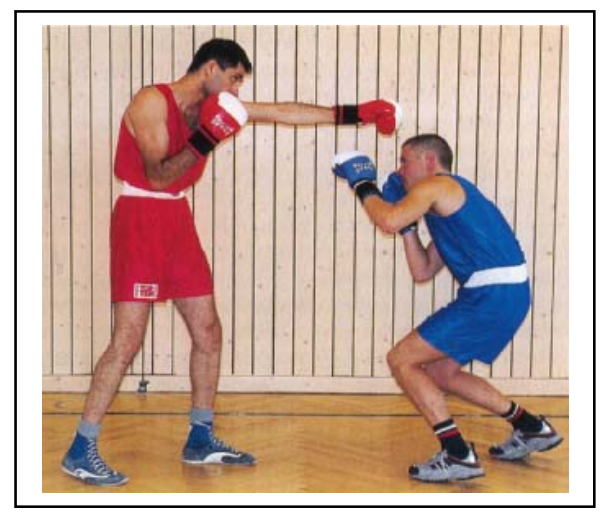

Abb. 28: Abducken nach unten

(nach: ELLWANGER; ELLWANGER, 1998, 97)

Diese Verteidigung ist eine einfache Finte mit den Beinen (Abtauchen). Verschieden ist nur die Leistungszeit (nach dem Schlag vom Gegner oder die Vorbereitung für die Schläge).

Rückneigen: Bei dieser Verteidigung gegen gerade Schläge in der weiten Distanz wird insbesondere das Rückneigen verwendet. Der gerade Stoß des Gegners zum Kopf wird durch Rückneigen des Oberkörpers in Verbindung mit einer Gewichtsverlagerung auf das hintere Bein vermieden. Diese Verteidigungsposition ist eine günstige Ausgangsposition für das Schlagen der geraden Schlagoder Führungshand. (vgl. FIEDLER, 1997)

Rollen: Dies ist eine Verteidigung gegen Kopfseitwärtshaken (links oder rechts). Durch das Bewegen des Oberkörpers mit der Schlagrichtung des gegnerischen Hakens werden Treffer vermieden oder zumindest in ihrer Schlagwirkung stark eingeschränkt. Kreisförmige Rollbewegungen werden durchgeführt, damit der Boxer unter dem Arm des Gegners hinweg tauchend wieder seine ursprüngliche Kampfposition einnimmt. Dabei wird das Körpergewicht entsprechend der Rollbewegung verlagert. Der Boxer muss während dieser Verteidigungshandlung auf die Vermeidung von Kopfstößen achten und die volle Kampfübersicht behalten. Die Rollbewegungen lassen sich sehr gut mit Seitwärtshaken verbinden. (vgl. SONNENBERG, 1989; ELLWANGER; ELLWANGER, 1998) 
Dies ist eine Verteidigung, bei der die einfache Finten mit dem Rumpf (Vorwärts ausweichen links, Vorwärts ausweichen rechts) ausgeführt werden kann.

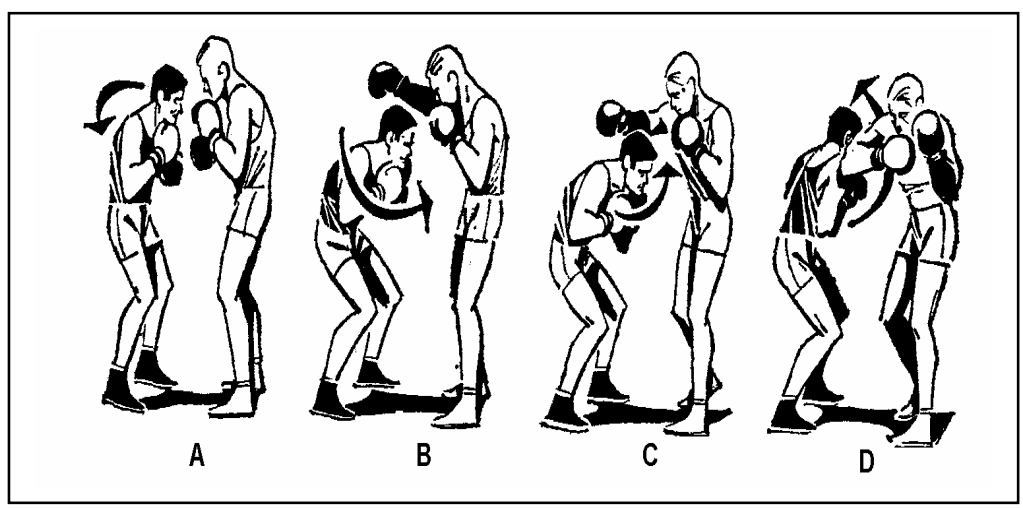

Abb. 29: Vermeiden des Seitwärtshakens mit der Schlaghand zum Kopf durch Abrollen nach rechts (nach: FIEDLER, 1997, 65)

Pendeln: Der Boxer führt die Verteidigung aus, um vorwiegend Seitwärtshaken zu vermeiden und um den Gegner die Vorbereitung seiner Angriffe zu erschweren. Erfahrene Boxer pendeln und rollen, wenn sie sich im Schlagbereich des Gegners (vorwiegend in der Halbdistanz) befinden, um kein feststehendes Ziel zu bieten und um die Pendelbewegungen als Auftakt für überraschende Angriffsaktionen (Angriffsvorbereitung) zu benutzen. (vgl. ELLWANGER; ELLWANGER, 1998)

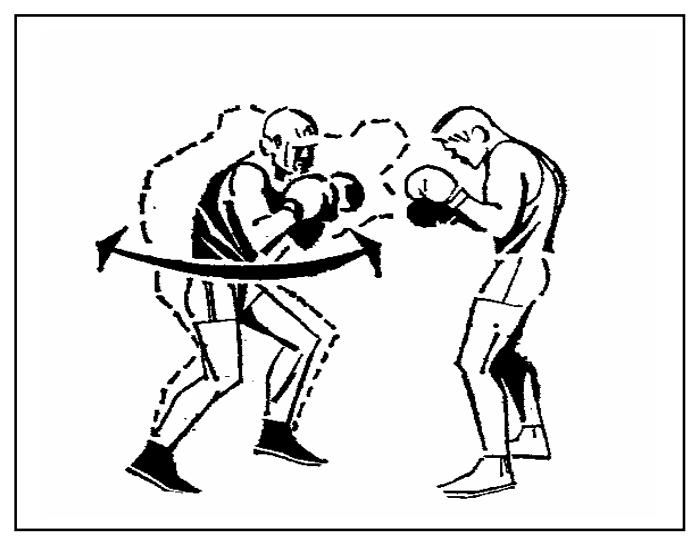

Abb. 30: Pendeln des Boxers im Schlagbereich (nach: FIEDLER, 1997, 66)

4. Die Ausweichbewegung: Diese Verteidigungsform enthält alle Verteidigungsaktionen und Reaktionen, bei denen der Boxer durch Schritte oder Sprünge (Beinbewegungen) seinen Standort verändert, um den gegnerischen 
Schlägen auszuweichen. Dazu zählen hauptsächlich der Rückschritt oder Sprung und der Seitschritt (Sidestep) oder Seitsprung. Im Verlaufe seiner Ausbildung lernt der Boxer noch eine ganze Anzahl von Varianten dieser Ausweichbewegungen (z. B. Schritt schräg nach hinten), die alle darauf gerichtet sind, schnell aus dem gegnerischen Schlagbereich zu gelangen und dabei eine günstige Angriffsposition beizubehalten. Im modernen Boxen dominieren die Ausweichbewegungen zur Seite. (vgl. OGURENKO, 1972; SONNENBERG, 1989)

Die effektive Technik in der Praxis, jedoch sehr selten zu beobachten, ist - die Technik, durch einen Sidestep, einen Schritt zur Seite, den Gegner ins Leere laufen zu lassen. Durch das Angreifen des Gegners wird der linke Fuß fast bis an den rechten herangezogen. Der Oberkörper wird - meist nur für kurze Zeit aus der Reichweite des Gegners gebracht, was eine sehr labile Stellung ergibt. Der rechte Fuß wird nach vorn rechts gesetzt und die linke Schulter über den Fuß gebracht. Mit einer Drehung des Oberkörpers zum Gegner hin wird der linke Fuß herangezogen: Deshalb steht Boxer neben dem Gegner und kann inn mit der Schlaghand, die eine gute Vordehnung hat, gut erreichen. (ELLWANGER; ELLWANGER, 1998)

Rückschritt: Dem Angriff des Gegners wird durch einen oder mehrere Schritte entsprechend der Vorwärtsbewegungen des Gegners nach hinten ausgewichen. Während des Ausweichens muss der Boxer auf eine gute Kopf- und Körperdeckung achten, weil er bei stürmischen Angriffen die Schläge nicht völlig vermeiden kann. In der Regel sollten die Rückschritte und eignen Schläge (aktive Verteidigung) verbunden werden. (vgl. FIEDLER, 1997) 


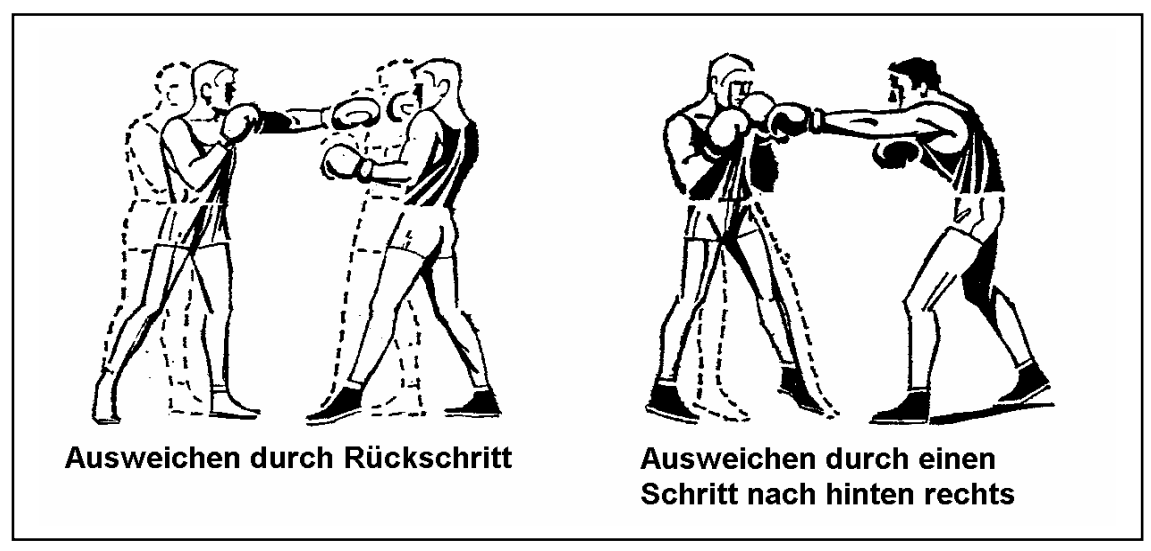

Abb. 31: Ausweichen durch Rückschritt (nach: FIEDLER, 1976, 86)

Rücksprung: Die Grundlage dieser Verteidigung basiert auf kräftigen Abdrücken mit dem Ballen des vorderen Fußes. Dabei gleiten die Füße flach über dem Boden. Die Kampfposition wird beibehalten und der Boxer kann nach dem Rücksprung zum Angriff übergehen (Gegenangriff). (vgl. SONNENBERG, 1993)

Der Boxer kann diese Verteidigung wie eine einfache Finte anwenden, weil sie ähnlich wie die einfache Finte mit den Beinen (Halber Rückwärtsschritt) ist.

Ausweichungen zur Seite: Diese Verteidigung zur rechten oder linken Seite bewirkt, dass der Boxer aus der gegnerischen Angriffsrichtung gelangt und bei besserer Ausführung gleichzeitig eine günstige Position für den eigenen Angriff schafft. Dieses aus der Linie gehen in Verbindung mit Sicherungsschlägen gehört zum Standardrepertoire eines Klasseboxers. (vgl. FIEDLER, 1997)

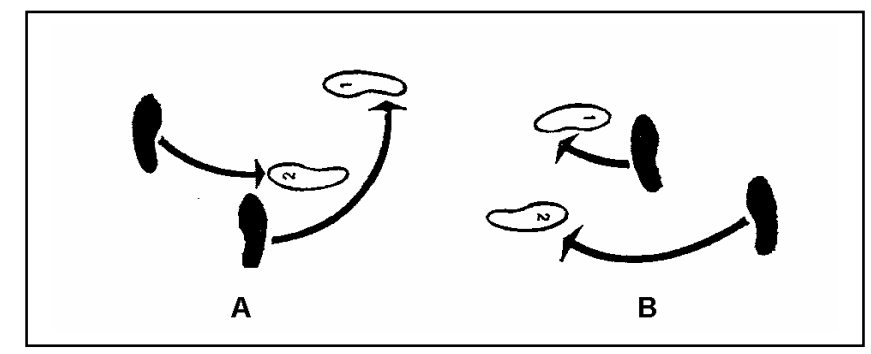

Abb. 32: Die Beinbewegungen beim Sidestep nach rechts $(A)$ und links $(B)$ (nach: FIEDLER, 1997, 46) 
Der Boxer führt im Prinzip eine Vierteldrehung nach seitwärts- vorwärts aus. Bei gemachtem Sidestep nach rechts erfolgt fast gleichzeitig mit dem Rechts- Seitwärts-Vorwärts-Setzen des rechten Fußes ein Umlauf auf beiden Fußballen um $90^{\circ}$, wobei der linke Fuß nachgesetzt wird. Der Boxer steht in Boxstellung seitlich vom Gegner und hat somit die Möglichkeit einen Seitwärtshaken oder Aufwärtshaken zu schlagen wenn die Situation im Halbdistanzbereich liegt. (vgl. SONNENBERG, 1989; ELLWANGER; ELLWANGER, 1998)

Abschließend sei zu den Verteidigungen noch gesagt, dass in der Wettkampfpraxis häufig zwei Verteidigungsmöglichkeiten miteinander verbunden werden, wie beispielsweise eine Parade und Ausweichbewegung oder Meidbewegung mit Deckungen.

\subsection{Taktik im Boxen}

Das wesentliche Mittel für die Boxkämpfer ist die Taktik, die sich bezüglich ihrer physischen, technischen und körperlichen Voraussetzungen kaum unterscheiden. Diese taktischen Möglichkeiten, die abhängig sind von der Intelligenz und Kreativität jedes Einzelnen, setzen die Boxer ein, um einen Wettkampf für sich zu entscheiden. Selbst vor brutalen Fouls, Bestechung, Manipulation und Doping wird nicht Halt gemacht. (vgl. BARTH, 1991)

Die wachsende Bedeutsamkeit boxsportlicher Gewinne erhöht die Gefahr unsportlichen Verhaltens und manipulierter Resultate. Neben dem Ermahnen der Sportler zu fairem und ehrlichem Verhalten müssen Regelungen getroffen und entsprechende Kontrollen ausgeübt werden, die es ermöglichen, im Wettkampf alle individuellen und kollektiven Leistungsvoraussetzungen für das Erreichen guter Resultate aufzubieten. (vgl. BARTH, 1990)

Taktik ist ein sinnvoller Einsatz der Technik in der jeweils gegebenen Situation. Dieser Einsatz setzt eine schöpferische Denktätigkeit voraus, die auf Grund von gespeichertem Wahrnehmungs- und Analysevermögen aus verschiedenen Entscheidungsalternativen die jeweils richtige Lösung herausfiltert. Dabei soll Instinkt, Vernunft sowie Realisierbarkeit der jeweiligen Entscheidung die Grund- 
lage jedes Handlungsplanes sein. Schöpferische Denkfähigkeit und individuelle Kreativität sind für die situative Anwendung und Umsetzung von Angriffs- und Abwehrtaktiken erforderlich. (vgl. SCHÖNBORN, 1990)

Das ausgeprägte Zusammenwirken der Boxer im Einzelsport wie in den Spielen ist durch einen Prozess des ständigen Aufnehmens, Widerspiegelns und durch die Rückwirkung auf das Aufgenommene geprägt. Die taktische Mitwirkung hängt vom adäquaten Aufnehmen einer Situation im richtigen Zeitpunkt und von der Einschätzung seiner eigenen und der Möglichkeiten des Gegners ab. Bei der Ausübung einer bestimmten Taktik ist eine verstandesmäßige Steuerung notwendig. Diese lässt sich so verdeutlichen: Der Boxer $X$ gibt dem Boxer $Y$ vor, nach einem bestimmten System zu kämpfen. Der Boxer $Y$ übernimmt das inm vorgegebene Modell und versucht, inm entgegenzuwirken. Die verstandesmäßige Steuerung besteht hier in der Vorstellung der vermuteten weiteren Handlung des Gegners und in den entsprechenden Gegenmaßnahmen. Den wesentlichen Kern der verstandesmäßigen Steuerung stellt die umfassende und äquivalente Einschätzung der gegnerischen Handlung dar. Im Sportspiel erfordert der verstandesmäßige Beitrag nicht nur das Erkennen des gegnerischen Spielmodells, sondern auch des konditionellen Zustandes, des technischen Könnens und des taktischen Vorgehens. (vgl. KELLER, 1976) Die immense Bedeutung der Taktik ist besonders für den Bereich des Boxens unumstritten. Aber auch in den kompositorischen Sportarten kann - besonders im Hochleistungsbereich - die Ausprägung des Leistungsfaktors Taktik zum Wettkampfentscheidenden Moment werden. (vgl. FRIEDRICH, 1984)

Wichtig ist methodische Wege zu einfachen Boxsystemen in Angriff und Verteidigung aufzuzeigen, wobei die Prinzipien "Vom Bekannten zum Unbekannten", "Vom Leichten zum Schweren" und "Vom Einfachen zum Komplexen" Berücksichtigung findet. (vgl. BARTH, 1991; BERGER, 1996)

Drei Teilbereiche stellen die komplizierte taktische Handlungsfähigkeit dar. Die taktischen Kenntnisse enthalten die Wettkampfregeln und allgemeine Erfahrungen zum optimalen Verhalten im Wettkampf. Die taktischen Fähigkeiten orientieren sich am Verlauf der Handlungsregulation und untergliedern sich entspre- 
chend in Wahrnehmungs-, Antizipations- und Entscheidungsfähigkeit. Taktische Fertigkeiten sind automatisierte Handlungsprogramme, die ohne bewusste Steuerungsvorgänge im Kampf als so genannte Assoziationslösungen abrufbar sind. (vgl. BARTH, 1995)

Um ein taktisches Anforderungsprofil erstellen zu können, muss man den Ablauf der Wettkampfhandlung analysieren. Vom Wesen einer allgemeinen Wettkampfhandlung ist in erster Linie das Typische der taktischen Handlungsfähigkeit abzuleiten. Dabei werden am Beispiel einer Kampfhandlung die an den einzelnen Phasen beteiligten Prozesse und Fähigkeiten und deren Erscheinungsformen, den verschiedenen relativ abgrenzbaren Struktureinheiten der Handlungsregulation zugeordnet.

Der Boxkampf verläuft im ständigen Wechsel der taktischen Haupthandlung Angriff und Verteidigung.

In der folgenden Abbildung sind die taktischen Haupthandlungen dargestellt. 


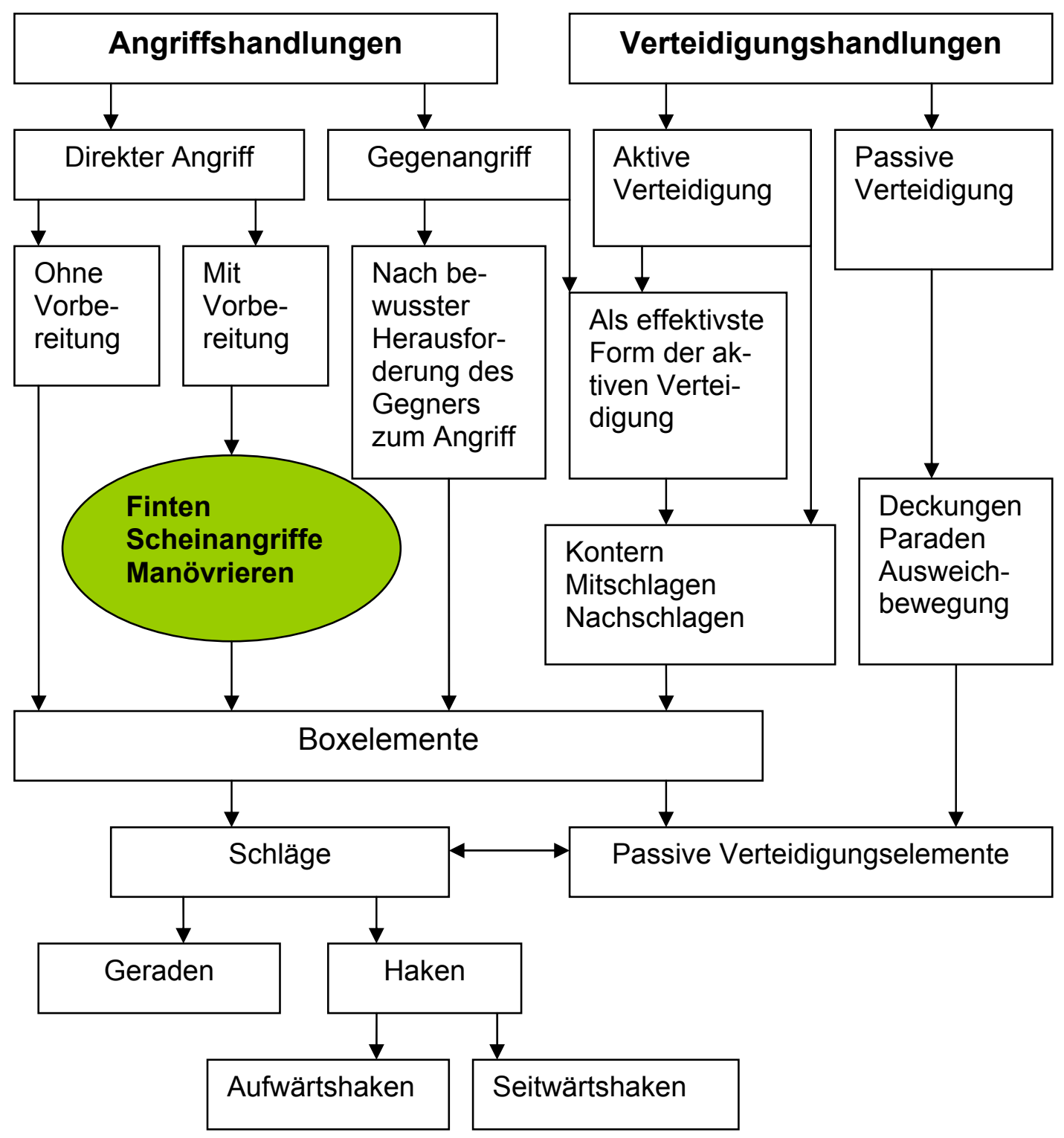

Abb. 33: Die Rolle der Finten bei den taktischen Haupthandlungen im Boxen (modifiziert nach: FIEDLER, 1997)

Die allgemeine Aufgabe der Taktik im Boxen ist für alle Stile dieselbe: Die rationelle Ausnutzung der taktischen Gelegenheiten mit wenig Kraftverlust zur Erreichung des Wettkampfsieges im Rahmen der Wettkampfregeln. Der Vergleich der jeweiligen Möglichkeiten der kämpfenden Seiten ist Teil des taktischen Wettkampfplanes. Die Möglichkeiten der Boxer umfassen u. a. das Bewegungsfertigkeitsniveau, das psychophysiologische Eigenschaftsniveau und die Kampfeinstellung. Der totale Druck ist für eine erfolgreiche Kampfführung unverzichtbar. Dieser muss während des gesamten Kampfes eingesetzt werden. 
Voraussetzung ist allerdings, dass der Boxer dem Gegner an spezieller Ausdauer überlegen ist und von dieser auch überzeugt ist. (vgl. ALICHANOV; SACHMURADOV, 1985)

Programmierte Instruktion ist eine Methode zur Vermittlung irgendeines Lerninhaltes. Ein nach dieser Methode aufbereiteter Lerninhalt wird Lernprogramm genannt. Diese Methode ist notwendig beim Boxen und mit einem Lernprogramm können die Boxer die Vermittlung richtiger Technik und Taktik erhalten. Mit dem Lernprogramm erweitern die Boxer systematisch ihr Wissen. (vgl. BRANDT; STAHL, 1984)

Bei Anfängern ist der Unterricht strategisch-taktischer Natur. Bei Fortgeschrittenen müssen die taktischen und technischen Lösungen in gegenseitigem $\mathrm{Zu}-$ sammenhang gesucht werden, wenn möglich, sollte immer die technische Schulung taktisch begründet werden. (BRECHBÜHL, 1990)

Die taktischen Grundlagen des Boxens mit Anfängern zu trainieren ist besonders wichtig, da der Boxsport auf ein sinnvolles Mindestmaß taktischen Verhaltens angewiesen ist. Die Einsteiger sollen die Grundregeln der Taktik in spielerischer Form früh erlernen. (vgl. VOGT, 1991)

Behandelte Situationen sind:

1. Verteidigung gegen die geraden Schläge,

2. Angriff mit den geraden Schlägen,

3. Gegenangriff mit den geraden Schlägen,

4. Einfache Finten.

Obwohl das Bewusstsein um die Bedeutung von Strategie und Taktik besonders in den Zweikampfsportarten in den vergangenen Jahren gestiegen ist, mangelt es an eindeutigen Definitionen, die als Forschungsgrundlage unerlässlich sind. Die Auswirkungen der vollzogenen Handlung sind als Feedback für die kommende Informationsaufnahme signifikant. Die Zweikampfsportarten als Teil der so genannten Open Fertigkeits-Disziplinen basieren theoretisch auf der Täuschung des Gegners als fundamentales Element der Taktik. 
Die Taktik wiederum wird per Definition der Strategie als übergeordnetem Handlungsschema unterstellt. Neben den grundlegenden Begriffen sind für die strategische und taktische Erarbeitung einer Handlungsanweisung im Boxen die Gesichtspunkte Kontrolle, Tempowahl und Automatismen zu berücksichtigen. Dem Leistungsniveau entsprechende Differenzierungen sind im Hinblick auf konkrete Trainingsaufgaben von enormer Bedeutung, wobei zu beachten ist, dass auch Anfänger eine strategische und taktische Anleitung benötigen. (vgl. TORAN, 1995) Als wichtige Methodik des taktischen Trainings wird ein häufiger Wechsel des Partners unter Berücksichtigung der Unterschiede ihrer Qualifikation und Besonderheiten ihrer Taktik hervorgehoben.

\subsection{Die taktische Führung des Kampfes}

Ausschlaggebende Faktoren im modernen Boxen sind die taktischen Kampführungen. Auswertungen vieler internationaler Meisterschaften ergaben, dass der Kreis der ausgezeichnet technisch und konditionell ausgebildeten Spitzenboxer ständig größer wird und im unmittelbaren Aufeinandertreffen die strategischtaktische Kampfgestaltung den Ausschlag über Sieg oder Niederlage gibt. Dabei erfordert jede auf den Sieg ausgerichtete Kampfkonzeption, selbst Kampf bestimmend zu sein. (vgl. RÄSCH, 1973; SONNENBERG, 1993; ELLWANGER; ELLWANGER, 1998 )

Kampf bestimmend zu sein heißt weiter, den Kampfverlauf so zu gestalten, dass die eignen boxerischen Mittel mit hochgradiger Effektivität eingesetzt werden können. Ob der Boxer dabei meist angreift oder sich z. B. nach bewusster Herausforderung des gegnerischen Angriffs durch Gegenangriffe entscheidende Vorteile erarbeitet, ist eine taktische Frage. (vgl. SONNENBERG, 1989; MÜLLER, 1991)

Das taktische Verhalten des Boxer hängt unter anderem von der Wahrnehmung der Umgebung, vom Verhalten des Gegners und vom eigenen Leistungsniveau ab. Bei der Taktikschulung sind folgende Eigenschaften vorrangig zu lösen: Entwicklung des Distanzgefühls, die Wahl des richtigen Zeitpunkts der 
Handlungsdurchführung, beides abhängig von der Konzentrationsfähigkeit und der Reaktionsschnelligkeit des Boxer. Voraussetzung einer effektiven Taktik sind ein hohes technisches und konditionelles Leistungsniveau sowie ein hohes Entwicklungsniveau der psychomoralischen und Willenseigenschaften - wie Mut, Initiative etc.. Wichtig bei der taktischen Vorbereitung ist die richtige Einschätzung des eigenen und des gegnerischen Leistungsvermögens, die Analyse der Wettkampfbedingungen, der Wettkampfstätte, des Klimas, der Schiedsrichter und des Zuschauerverhaltens. Im Taktiktraining sind bestimmte Wettkampfsituationen zu simulieren, welche die adaptiven Fähigkeiten des Organismus erhöhen. (vgl. LANGHOFF; SCHOTT, 1983)

Das Ausschlaggebende ist jedoch, dass der Boxer selbst die Initiative ergreift, um dem Gegner eine Kampfführung aufzuzwingen, die den eigenen Fähigkeiten und Fertigkeiten am meisten entspricht und den effektivsten Einsatz der individuellen boxerischen Mittel gestattet. Dieses für den Erfolg in den internationalen Boxkämpfen notwendige Kampf bestimmende Verhalten äußert sich im Wettkampf wie folgt:

— dauerndes Kontakthalten mit dem Gegner in Verbindung mit einer guten Kampfübersicht,

- Kontrolle des Gegners durch Stören, Bedrängen und „Binden” der Schlaghand sowie Vorbereitung und Durchführung eigner Wirkungshandlungen,

- folgerichtiges Ausnutzen jeder Gelegenheit. Der Boxer muss dazu seine Führungshand variabel einsetzen, seine Aktionen verschleiern und durch aktive Verteidigungshandlungen die Kampfinitiative übernehmen. (vgl. FIEDLER, 1976; STAUFFER, 1993)

Man kann bei der taktischen Kampfführung nachstehende fehlerhafte Varianten beobachten: Mangelnde Aktivität - defensive Kampfführung oder übertriebene Aktivität - bedingungsloses Suchen des Schlagabtausches im Angriff. (FIEDLER, 1997)

Die Erscheinungsformen für die mangelnde Aktivität und defensive Kampführung sind $\mathrm{z}$. B.

- geringe Aktivität in der 1. Runde des Kampfes, 
- kontern des angreifenden Gegners aus der Defensive, ohne nach dem erfolgreichen Konter einen Gegenangriff zu starten,

— das ständige Führen des Kampfes im Rückwärtsgang,

— isoliertes Anwenden passiver Verteidigungsbewegungen zur Vermeidung gegnerischer Treffer,

- das fälschliche Anpassen der eigenen Kampfesweise an die des Gegners. (vgl. RÄSCH, 1973; SONNENBERG, 1978; ELLWANGER; ELLWANGER, 1998)

\subsection{Das Verhalten im Boxring}

Der Boxer wird durch einen Boxring in seinen Bewegungen begrenzt, was sich in der technisch-taktischen Entwicklung widerspiegelt. Die ungewohnte Situation erfordert nunmehr - neben der Beobachtung der Aktionen des Gegners - zusätzlich eine räumliche Orientierung. Abgesehen von den strategisch-taktischen Grundeinstellungen eines Boxers und dem sich ständig ändernden Kampfgeschehen, gibt es im Geschehen eines Wettkampfes sich häufig wiederholende Situationen, die von dem Aktiven eine ganz bestimmte taktische Verhaltensweise verlangen. Diese typischen, häufig wiederkehrenden Situationen fassen wir unter dem Begriff ,,taktische Standardsituationen” zusammen. Für das Verhalten und ihre Lösung gibt es bestimmte taktische Grundregeln, die der Boxer in Theorie und Praxis beherrschen muss. Bei diesen taktischen Regeln unterscheiden wir zwischen Verhaltensweisen gegen bestimmte Kämpfertypen und den Verhaltensweisen in den eigentlichen taktischen Standardsituationen. (vgl. FRIEDLER, 1976; MÜLLER, 1991; ELLWANGER; ELLWANGER, 1998)

Je vielfältiger die Kenntnisse über ständig wiederkehrende Situationen eines Kampfes sind, umso einfacher wird es, den taktischen Gegebenheiten Rechnung zu tragen. Der Boxeinsteiger muss lernen, den Übungspartner durch Boxaktionen aus dem Ringmittelpunkt zu verdrängen. Das kann erfolgen durch:

- ständige Aktivität der Führungshand,

- Schlag-Kombinationen, die schnell Aufeinanderfolgen oder 
- Einfache Finten z. B. Ausweichen mit dem Rumpf und sofortiges Zurückgehen auf die vorher verlassene Position.

Das erfordert:

- das Einhalten vorgegebener Techniken,

- das dauernde Suchen des Kampfes sowie

- die Beachtung des eigenen Standortes im Boxring.

Das wiederum ist nur möglich, wenn die sportlichen Belange - gegenüber den emotionalen Regungen von Treffen und Getroffen werden - überwiegen: Hier beginnt das sportliche Boxen. (vgl. FRIEDLER, 1976; SONNENBERG, 1989)

Den Gegner aus der Ringmitte zu drängen wird im Boxring als eine der ersten Übungen betrieben. Der aktive Kämpfer hat dann den Rücken frei und ist in der Lage, sofort zurückzugehen.

Das erfordert:

- Einhalten der vorgegebenen taktischen Aufgabe,

- Konzentration auf diese Aufgabe unter Vernachlässigung von Techniken sowie

- bewusstes Anwenden von Grundstößen und flexibler Beinarbeit.

Das ermöglicht:

- den Gegner mit einem weiteren Schritt am Seil zu stellen, so dass er nur noch nach rechts oder links ausweichen kann, während er mit dem Rücken am Seil steht, so wird er gezwungen, in die Richtung auszuweichen, die für die Entfaltung der eigenen Aktivität am günstigsten ist,

- den Gegner in eine Ecke zu drängen, wo er keinen Spielbereich für Ausweichbewegungen hat,

- den Gegner dort so lange mit der Führungshand zu beschäftigen, bis eine Deckungslücke sichtbar wird. (vgl. SONNENBERG, 1989; ELLWANGER; ELLWANGER, 1998)

Den Kreis »offen« zu halten versteht man als offensive Taktik, in Grobform beherrscht, soll weiter geübt werden, sich nicht an die Ringseile drängen zu lassen. Um von den Ringseilen weg- oder aus der Ringecke herauszukommensind mehrere taktische Maßnahmen möglich: 
Wenn der Gegner zum Stoß ansetzt, wird einem eigenen Stoß erwidert, der immer mit einer schnellen Bewegung nach vorn verbunden ist. Es wird ein Angriff angetäuscht und gleichzeitig versucht, nach rechts oder links zur Ringmitte zu kommen. (vgl. ELLWANGER; ELLWANGER, 1998)

Es sollte versucht werden, nach einem Sidestep mit Schlag oder Verteidigungshandlungen den Schlag des Angreifers zu binden, um auf diese Weise zur Ringmitte zu kommen. Ein kraftvoller Schlag kann die Befreiung aus der Ringecke ermöglichen. Auf die Deckung des Gegners gerichtet ist er ein Überraschungseffekt, um für den Bruchteil einer Sekunde alle weiteren Aktionen zu unterbinden: In dieser Zeit muss die Ringecke bereits verlassen sein. Die Aktion könnte wie folgt aussehen: Pendeln mit dem Oberkörper nach vorn links - nach vorn rechts - Stoß mit der Schlaghand - Schritte nach vorn rechts - Halber Vorwärtsschritt (Finten) - Kurze Gerade (Finten) - linke Gerade zur Absicherung.

Das Verhalten ist bedingt durch die ungleichen Gegner. Hierzu gehören neben dem Anpassungsvermögen wichtige Kenntnisse von der Führung des Kampfes mit größeren, kleineren, schlagstarken, ebenbürtigen Gegnern und Rechtsauslegern. Bei schnellen Gegnern ist die weite Distanz oder der Nahkampf eine probate Lösung.

Der Wettkampf besitzt auch neben unterschiedlicher Gegnerschaft selbst eine Reihe von Kampfmomenten, die sich ständig wiederholen. Die sinnvolle, taktische Lösungsvariante der Aufgabe muss jedem Sportler bewusst sein.

Die am häufigsten auftretenden Situationen sind:

— das Lösen vom Gegner,

- das Unterbinden ungünstiger Kampf-Distanzen,

— das Überbrücken kritischer Situationen wie: verlorene Übersicht, Groggyzustand,

— das Verhalten nach erzieltem Niederschlag,

- das Verhalten nach erhaltenem Niederschlag. (vgl. OGURENKO, 1972; FRIEDLER, 1997)

Die Möglichkeiten und Varianten des Verhaltens, die sich durch den Boxring als Wettkampfplatz ergeben, müssen auch eine Grundlage für die taktische Konzeption sein. Die Kenntnis und Einhaltung der Wettkampfregeln, die Beachtung 
der Ringrichterkommandos und das Ausnutzen der sich daraus ergebenden Möglichkeiten, lässt zahlreiche nachteilige Handlungen vermeiden.

Die Berücksichtigung und das Einbeziehen klimatischer Verhältnisse und der örtlichen Gegebenheiten ist bei der taktische Planung unerlässlich, z. B. Standort des Boxringes, Halle oder Freiluft, Temperatur, Heim- oder Auswärtsstart.

1. Verhalten beim Kampf gegen größere Boxer: Boxer ungleicher Körpergröße in derselben Gewichtsklasse sind nicht die Regel. Meistens sind es größere Boxer mit einer guten Reichweite und „schnellen Beinen«. Wie sollte gegen sie geboxt werden?

Die größeren Boxer sind ständig mit der Führungs- und der Schlaghand zu beschäftigen: So können sie keinen schnellen Angriff anwenden. Der so aufgebaute Druck soll sie in die Ringecke, beziehungsweise an die Seile drängen. Durch schnelle Schlagserien (leichte, lockere Schläge) arbeitet man sich an die richtige Distanz heran. Erst jetzt wird versucht, gezielte und harte Treffer anzubringen. Es folgen leichte Stöße zur Absicherung. Dann erfolgt ein neuer Serienangriff.

Der Boxer verwendet die einfache Finten mit dem Rumpf und mit den Beinen oder kombinierte Finten mit den Beinen und dem Rumpf, um den Gegner in Halbdistanz oder Nahkampf zu positionieren. Dann erfolgt der Angriff.

Der kleine Boxer muss lernen, gegen größere Boxer zu kämpfen, auch wenn dieser den Kampf auf der langen Distanz bevorzugt. Ihm stellt man deshalb folgende Aufgaben: Die Angleichung durch seitliches Ausweichen links (Schritt vorwärts oder links seitwärts) und rechts (Schritt vorwärts oder rechts seitwärts), um den Schlägen des Gegners zu entgehen, Angriff im günstigen Zeitpunkt mit Schlägen zum Kopf oder Körper, Eintritt in die Halbdistanz oder Nahkampf mit einer Schlagserie, um die Distanz nicht zu verlängern und den Gegner möglichst in die Ringecke oder an die Seile zu drängen, Herausforderung des Gegner zum Schlag durch geringes Zurückweichen und leichtes Öffnen der Kopfdeckung. Hat der Gegner geschlagen, Annäherung durch seitliches Ausweichen (Finten mit dem Rumpf) und Abducken (Finten mit den Beinen), so dass der Gegner ins Leere schlägt. Dieser Augenblick wird ausgenutzt, um ei- 
nen Gegenangriff mit einer Schlagserie zu entwickeln, nachher darf der Nahkampf nicht unterbrochen werden.

Nimmt der Gegner eine Verteidigungsposition ein, ohne zu schlagen, oder versucht er, den Boxer zu klammern, dann müssen unerwartete Aufwärtshaken zum Kopf zwischen seinen Armen hindurch geschlagen und der Nahkampf fortgesetzt werden. (vgl. OGURENKO, 1972; ELLWANGER; ELLWANGER, 1998)

2. Verhalten beim Kampf gegen kleinere Boxer: Die Führungshand des kleineren Boxers sollte an der so genannten "Leine“ geführt werden. Aus der Führungshand wird ständig Kontakt zu den Fäusten des kleineren Gegners gehalten. Beginnend, wenn er Pendelbewegungen ausführt oder beide Beine auf gleicher Höhe hat, wird die Schlaghand eingesetzt. Diese sollte immer auf das Schlüsselbein zielen, da der Kopf bei schnellen Bewegungen nach links und rechts nur schwer zu treffen ist. Beim Ausweichen bewegt man sich immer seitlich, meist zur Führungshand hin. Kleinere Boxer sollte man nie direkt von vorn, sondern nur seitlich von vorn angreifen. Beispiel: Eigene Führungshand gegen die Führungshand des kleineren Gegners mit Schritt rechts und Stoß rechts Nachziehen des linken Beins.

Der Boxer verwendet die einfache Finten mit den Beinen, den Armen oder kombinierte Finten mit den Beinen und den Armen, dann erfolgt in der Weitdistanz der Angriff. (vgl. FIEDLER, 1997; ELLWANGER; ELLWANGER, 1998)

Folgende Aufgaben empfehlen sich bei Boxern, denen der Nahkampf nicht liegt oder die nicht die passende Erfahrung haben: Schnelles Manövrieren und Finten durch den Ring in verschiedenen Richtungen, wobei stets die lange Distanz gewahrt und dem Gegner durch gerade Stöße keine Gelegenheit zur Annäherung gegeben wird. Im Augenblick der Annäherung wird ein kurzer Schlag (Einfache Finten mit den Arme) oder eine schnelle Schlagserie angebracht, um daraufhin schnell (mit Geraden der linken Hand) auf die lange Distanz zurückzuweichen. Ausnutzung der Verteidigungspositionen unter Anwendung des Stoppens durch Auflegen der Unterarme, damit der Gegner während der Angleichung keine Aufwärtshaken schlagen kann, nun drückt der Boxer die Brust 
zum Gegner (ohne diesen zu halten) und legt die Arme von unten oder oben (ebenfalls ohne zu klammern) auf. (vgl. OGURENKO, 1972)

3. Verhalten beim Kampf gegen schlagstarke Boxer: Es gibt kräftige Boxer, die für die lange Distanz nicht schnell genug sind und deshalb im Mittelring, an den Seilen oder zu Ecke neigen, um ihre starken Schläge auszuführen. Der schlagstarke Boxer verwendet die schnelle Fortbewegung und schnelle Wechselschläge, die Verteidigung, die Finten, und den ständigen Sichtkontakt während des gesamten Kampfes. Der Boxer muss auch den Nahkampf und den Boxkampf in der Ecke vermeiden, wenn es möglich ist.

Gegen derartigen Gegner muss vor allem eine schnelle und wendige Kampfführung angewandt werden. Der gegnerische Angriff darf keinesfalls mit einem Gegenangriff beantwortet werden, der Schlagtausch ist zu meiden. Der Vorteil der Kraft kann nur durch eine Zermürbungstaktik aufgehoben werden, bei der es auf Geduld und Schnelligkeit ankommt. Für den Kampf gegen derart schlagstarke Boxer ist der Angegriffene taktisch zu schulen mit dem Ziel: Den Gegner nicht ständig auf der nahen Distanz handeln zu lassen, er muss den Kampf abbrechen, während er mit kurzen Schritten rückwärts oder seitwärts geht, um dann langsam wieder zum Nahkampf zurückzukehren und unerwartet eine oder zwei Schlagserien anzubringen, bevor der Gegner seine Kampfhandlungen erneuern kann. Es ist ein kombinierter Kampf zu führen, während man dauernd die Distanzen wechselt, so dass der Gegner sich nicht auf diese einstellen kann. Der Boxer muss den typischen vorwärtsgerichteten Annäherungsdrang insofern auszunutzen, als er den Gegner in die Seile fallen lässt und mit einer Schlagserie eindeckt, bevor dieser sich sammeln kann und in eine günstige Kampfposition zurückkehrt. (vgl. OGURENKO, 1972; FIEDLER, 1997)

\section{Verhalten beim Kampf gegen den Gegenangriff bevorzugenden Boxer:}

Der Gegenangriff ist beste Methode zum Wettkampfsieg, wenn der Gegner der den Gegenangriff bevorzugt, angreifen sollte. Der Angegriffene kann viele wechselnde Finten verwenden, damit er dementsprechende Schlagserien anwenden kann. 


\section{Verhalten beim Kampf gegen die einen Spezialschlag bevorzugenden}

Boxer: In der Mehrheit der Kämpfe richten die Boxer, die mit einem Spezialschlag arbeiten, ihre Taktik auf den Einsatz dieses Schlages aus. Bedeutend ist es, diesen Schlag zu erkennen und zu wissen, in welcher Situation er geschlagen wird, z. B. ob er bevorzugt in der Verteidigung oder im Angriff eingesetzt wird. Das ist durch entsprechende Taktik zu erkunden. Dafür bieten sich als Möglichkeiten an:

— Der Gegner wird bedrängt und deshalb gezwungen, diesen Schlag möglichst oft einzusetzen. Er kann im Moment leicht erkannt werden. Nach diesem Schlag muss ein Nachschlag oder ein Gegenangriff erfolgen,

- Sind die Einsatzvarianten bekannt, so wird versucht, dem Schlag zuvorzukommen, immer, entsprechend der Notwendigkeit, mit einem Mitschlag oder einem den Angriff einleitenden Schlag. (vgl. FIEDLER, 1997)

6. Verhalten beim Kampf gegen ebenbürtige Boxer: Es gibt den Kampf mit einem Gegner, der technisch und taktisch mindestens ebenso gut ausgebildet ist und etwa über die gleiche Konstitution und Kondition verfügt. Hier muss der Boxer sich taktisch besonders gut vorbereiten. Die Willensvorbereitung muss auf größte Entschlossenheit und Aufmerksamkeit ausgelegt werden und der Boxer darf dabei niemals Verkrampfung im Kampf aufkommen lassen. In den Kampfpausen kommt es darauf an, die Muskeln zu entspannen, um die größere Schnelligkeit im Kampf zu besitzen. Bewegungsschnelligkeit allein entscheidet nicht, es kommt auch auf eine schnelle Kampfführung und den schnellen Wechsel der Verfahren und Distanzen an. Einen gleichwertigen Gegner kann man nur bezwingen, wenn man ständig vielseitig handelt und die Distanz gerade dann wechselt, wenn der Gegner es am wenigsten erwartet. (vgl. OGURENKO, 1972; SONNENBERG, 1993)

Der taktische Plan muss danach heißen: Kombinierter Kampf mit vielen Finten, Ablenkungsmanövern und Geschwindigkeitswechseln und in der Zwischenzeit maximal schnelle Nahkampfoperationen. Vor allem muss der Boxer sich einige technische und taktische Neuheiten einfallen lassen, und keinesfalls darf der Boxer den Kampf so führen, wie der Gegner es erwartet. Dieser wird sich natürlich auch entsprechend vorbereiten. Deshalb kommt es darauf an, den Kampf 
von Beginn an schnell und energisch zu führen, um so den Gegner aus der Zurückhaltung zu locken und dabei besser seine Absichten zu erkennen.

7. Verhalten beim Kampf gegen rechtsauslegende Boxer: Der angreifende Rechtsausleger wird mit der störenden Führungshand bedrängt. Die Schlaghand deckt Körper und Kinn. Dadurch wird der Gegner gezwungen, seine Schlaghand verstärkt einzusetzen. Der Schlagansatz kann jetzt leicht erkannt werden. Es gilt nun, dem Schlag des Rechtsauslegers mit einer rechten Gerade zuvorzukommen. Der Gegner wird bedrängt mit der störenden Führungshand, die Schlaghand wird folgerichtig zum Körper eingesetzt. Dem Rechtsausleger wird damit die Möglichkeit genommen, seine Linke wirkungsvoll einzusetzen, Ausweichbewegungen erfolgen nach vorn links, um der Schlaghand kein einfaches Ziel zu bieten. Die eigene Schlaghand sollte möglichst viel eingesetzt werden und der Schlaghand des Gegners zuvorkommen. Wirkungsvolle Einzelschläge sind die rechte Gerade und der linke Haken zum Kopf des Gegners. Es empfiehlt sich auch, beide Schläge als Zweierkombination einzusetzen. Es gilt auch, Finten anzuwenden, um die Aufmerksamkeit in eine falsche Richtung zu lenken. Deshalb sollte der Boxer bereits im Rahmen des Trainings gegen Rechtsausleger antreten und spezifische Kampfverfahren entwickeln, die ihm technisch und taktisch einen Widerstand in der unterschiedlichen Distanz gegen Rechtsausleger erlauben.

8. Verhalten beim Kampf gegen die schnelleren Boxer: Das Ziel ist es, das Tempo des schnelleren Gegners zu vermindern, um die eigene Kampfkonzeption durchzusetzen. Der Gegner strebt in erster Linie Verwirrung durch eine hochgradige Aktionsdichte an. In der Mehrzahl verfügt er über keinen harten Schlag. Der Boxer muss harte Schläge, viel Fortbewegung und viele Fintenarten einsetzen und bestrebt sein, die Aktionsdichte der gegnerischen Schläge zu mindern. 
Zur Bekämpfung bieten sich folgende Varianten an:

- Durch ein ständiges Bedrängen und den Einsatz harter Schläge zum Körper wird das Tempo für den Gegner noch verschärft. Ist das Tempo ruhiger geworden, müssen auf den Gegner passende Konzeptionen eingesetzt werden.

- Den Aktionen des Gegners sind akzentuierte und wuchtig ausgeführte Kombinationen entgegenzusetzen.

- Der Boxer muss versuchen den Gegner am Seil oder in der Ringecke zu stellen, um seinen Aktionsradius einzuengen. (vgl. FIEDLER, 1997)

9. Verhalten beim Kampf gegen die Distanzboxer: Das Ziel ist das Durchbrechen der Distanz und das Stellen des Gegners, das "Hineinschieben“ in den Gegner, gestützt durch die sperrende Führungs- und Schlaghand. Den Distanzhaltenden Schlägen des Gegners darf sich kein Ziel anbieten. Er wird an die Seile gedrängt und der Kampf wird in der Halbdistanz fortgesetzt. Das Bedrängen muss innerhalb der Reichweite des Gegners durchgeführt werden. Unter Bedrängungen verstehen wir Stöße, die nicht mit voller Streckung des Armes verbunden sind. Die Stöße können deshalb schneller ausgeführt werden. Da die Arme nicht voll gestreckt werden, ist der Kraftverbrauch geringer. Die Stöße sind von Anfängern leichter zu erlernen. Doch die Gesamttechnik ist schwieriger. Man muss mit Stößen in der Halbdistanz näher an den Gegner herangehen: Das ist aber nur unter bestimmten, vorher geplanten und trainierten Bedingungen möglich. In der Halbdistanz sorgt die Boxhaltung dafür, dass beide Hände einen gleich langen Weg zum Ziel haben. Der Oberkörper ist somit fast frontal auf den Gegner ausgerichtet, das Kinn ist an die Brust gezogen und die Arme liegen eng am Körper an. Je nach Technik ist die Beinarbeit unterschiedlich. Am verbreitesten sind Diagonal- oder Passgang in Verbindungen mit Stößen. Wer aber vor allem das Körpergewicht einsetzen will, kommt mit dem Wechselschritt besser zum Ziel.

Durch druckvollen und mit hochgradigem Einsatz geführten Gegenangriff, nach erfolgter Verteidigung oder nach Fehlschlägen des Gegners, durch wellenförmige Angriffe, unterstützt von rollenden Bewegungen, Finten und pendelnden Oberkörperbewegungen wird der Gegenangriff geführt. Dabei ist zu beachten, 
dass nicht alle Angriffe einfach durchgeführt werden und die Geschwindigkeit des Angriffs sich wesentlich von der Geschwindigkeit des Kampfes unterscheiden muss.

Der Boxer führt die einfachen Finten, die kombinierten Finten mit den Beinen und mit dem Rumpf aus, um eine Nahkampfsituation zu schaffen. Dann kann er die Seitwärtshaken, Aufwärtshaken und Schlagserien schlagen. (vgl. STAUFFER; FRIEDLER 1997; SONNENBERG, 1993; ELLWANGER; ELLWANGER, 1998)

10. Verhalten beim Kampf gegen die Nahdistanzboxer: Der Nahkampf ist im Amateurlager selten zu sehen - denn man muss nahe an den Gegner herangehen, Körperkontakt mit dem Boxhandschuh herstellen, Stöße an Kopf und Körper anbringen und dann wieder, ohne getroffen worden zu sein, außerhalb seiner Reichweite gelangen. Diese Kampfform wird besonders von kleineren Boxern, explosiv handelnden und den Boxern mit guter Kondition und hochgradiger Beweglichkeit des Oberkörpers bevorzugt.

Wenn der Boxer gegen einen Nahdistanzgegner boxt, sollte er die einfachen Finten, die kombinierte Finten mit den Beinen und den Arme ausführen, weil der Nahdistanzboxer die Rumpfsbewegungen sehr gut ausführen kann.

Ziel ist das Einhalten der weiten Distanz und das Unterbinden der Halb- bzw. Nahdistanz, ständiges Kontakthalten zum Gegner mit der Führungshand und nur kurzes Halten. Es darf ihm kein Platz zur Angriffsentwicklung gelassen werden. Entscheidende Bedeutung kommt dabei der Führungshand zu. Sie erfühlt im gewissen Sinne die Distanz, leitet die Angriffe ein, bereitet den Einsatz der Schlaghand vor und wird bei variablem Einsatz zur entscheidenden Waffe. Die Distanz wird noch vergrößert, einmal, um eine bessere Übersicht zu bekommen, zum anderen, um dem Gegner die taktische Einstellung zu erschweren. In dieser Distanz sind schnelle Gegenangriffe und überraschende Angriffe sehr wirkungsvoll. Eine schnelle, aber rationelle Beinarbeit ist Voraussetzung dafür. (vgl. FRIEDLER, 1997; ELLWANGER; ELLWANGER, 1998) 
Der Gegner wird durch Zurückweichen in den Konter gezogen. Er muss zum Angriff verleitet werden. Dabei ist es günstig, sich ans Seil oder in die Ecke manövrieren und Finten zuzulassen. Dort erfolgt mit größter Wahrscheinlichkeit der Angriff. Diese Variante setzt ein gutes Anpassungsgefühl und Beobachtungsfähigkeit voraus. Da der Schlagbeginn und das Auftreffen unmittelbar aufeinander folgen, steht nur eine sehr kurze Reaktionszeit zur Verfügung. Deshalb ist es für die Gegner solcher Boxer besonders wichtig, die Bewegungen des Kontrahenten vorauszuahnen und das setzt Technikkenntnisse und Bewegungserfahrung voraus. (vgl. OGURENKO, 1972 ) 


\section{Die Täuschung im Boxsport}

Betrug, Lüge, Bluff und Imitationstäuschung sind nur in seltenen Fällen Gegenstand der Praxisliteratur (Ausnahmen: FRANCIS, 1977; SCHIEFLER, 1982). Einige Beispiele sollen die Anwendung zeigen:

- Betrug ist ein so weit verbreitetes und vielschichtiges Phänomen im Boxsport, dass seine medizinischen, soziologischen und die psychologischen Aspekte ein interessantes Untersuchungsfeld darstellt. Einige prinzipielle Varianten des Betruges sind:

1. Manipulation von Wettkampfergebnissen durch von Buchmachern bestochenen Boxer zum Zweck der Beeinflussung von Wettauszahlungen. (LEONARD, 1984).

2. Beeinflussung von Ergebnissen durch Gebrauch von Anabolika (LAMB, 1984), Dopingmitteln und / oder Drogen (z. B. SMITH, 1983).

- Bluff im Boxsport lässt sich der Bluff anhand des folgenden prominenten Beispiels verdeutlichen. Vor dem Kampf um die Boxweltmeisterschaft 1974 kündigte Mohammed Ali einen K.-O. Sieg über den K.-O. Spezialisten und viel stärker eingeschätzten George Forman an und hielt sein Wort. Jeder hatte Alis Ankündigung für den - wenn auch schlechten - Bluff gehalten und nicht die dadurch erzielte Wirkung gesehen. Alle, einschließlich Forman, glaubten sich der Schwäche Alis sicher zu sein und wurden dadurch zu unvorsichtig (vgl. BOWYER, 1982).

- Mit Täuschungsaufstellungen und Finten sind wir jetzt bei den "traditionellen", wettkampfinternen Täuschungshandlungen, die auch in der Praxisliteratur besprochen werden. Beispiele für Täuschungsaufstellungen sind Positionstäuschungen im Rahmen der Doppeltaktik im Boxen, zum Beispiel kombinierte Finten u. a.. (vgl. HESS, 1982; PAPAGEORGIOU; SPRITZLEY, 1986) 
Finten haben den größten Stellenwert innerhalb der genannten Täuschungshandlungen. Sie werden auch am häufigsten angewandt.

Folgende Gesichtspunkte sind von Interesse:

1. Bedeutung der Finten für den Wettkampf-/Spielerfolg,

2. Zweck des Einsatzes,

3. angestrebte Wirkung und

4. eingesetzte Mittel.

\subsection{Theoretische Betrachtung von Täuschungshandlungen im Boxsport}

Dieser Teil der Arbeit dient dazu, alle Täuschungssituationen im Boxen auf der Basis bestehender theoretischer Erkenntnisse so weit wie möglich zu analysieren und Fragestellungen für die anschließende Fallstudie zu formulieren.

Auch für eine Untersuchung von Täuschungshandlungen im Boxsport gilt, dass die handlungstheoretischen Konzepte die vielversprechendsten Ansätze zu einer ganzheitlich integrativen Betrachtung darstellen. (vgl. MECHLING, 1984) Der Nachteil dieser Ansätze ist, dass sie dem Leistungsbestimmenden Anteil der energetischen Grundlagen nicht gerecht werden und im Detail einen geringen Erklärungswert haben. Dies ist jedoch nicht so gravierend, wenn, wie hier, durch die Handlungstheorie nur der Analyserahmen festgelegt wird. Dieser Gedanke ist zwingend, weil das Täuschen in allen Varianten Handeln per Definition bedeutet.

Unter Handeln versteht man dabei:

1. Einen - Beziehungen stiftenden - Prozess,

2. der von Wissen gespeichert ist,

3. damit ein kognitives Geschehen repräsentiert,

4. das sich in einem Individuum vollzieht,

5. das in einem Milieu eingebettet ist,

6. diejenige Teilmenge an Verhaltensweisen, die

7. von Zielen und

8. Strategien gesteuert, 
9. die reguliert,

10. beabsichtigt sind

11. und in hohem Maße bewusst verlaufen;

12. die Bewältigung einer Aufgabe, damit verbundener Anforderungen und ihrer Beanspruchung. (vgl. FUHRER, 1984)

\subsection{Handlungsregulation bei Täuschungshandlungen im Box- sport}

Bei Täuschungssituationen im Boxen ist sowohl die Handlungsregulation des Täuschenden als auch dessen, der der Täuschungshandlungen ausgesetzt ist, von Interesse. Muss der Täuschende seinerseits mit einer Gegentäuschung rechnen, gelten für ihn diejenigen Aussagen zur Handlungsplanung und -regulation, die den möglichen Getäuschten betreffen. Wird in Zukunft sonst vom Täuschenden gesprochen, dann beinhaltet dies - wenn nicht explizit anders gesagt - ein Handeln ohne die Gefahr, einer möglichen Gegentäuschung ausgesetzt zu sein.

Vor den Täuschungssituationen mit Finten, Täuschungsaufstellung und Imitationstäuschung wird der Bluff als verbale Täuschungshandlungen besonders zur inhaltlichen Kontrastierung erwähnt. Für die theoretische Analyse werden die Prozesse der Orientierungs- und Entscheidungsregulation zusammengefasst (und die der Ausführungs- und Kontrollregulation). Der Grund dafür ist, dass die tatsächlich interessierenden entscheidungvorbereitenden Prozesse auch in Täuschungssituationen zeitgleich mit der Orientierungsregulation ablaufen oder ihr sogar vorangehen. (vgl. KONZAG; KONZAG, 1980)

\subsection{Orientierungs- und Entscheidungsregulation bei Täu- schungen im Boxen}

Die Orientierungsregulation umfasst alle jene psychischen Prozesse, die der Aneignung äußerer und der Reproduktion innerer Informationen dienen, die für die Erarbeitung eines Handlungsprogramms notwendig sind. Grundlage der Orientierungsregulation sind die sensorische Informationsaufnahme und die In- 
formationsverarbeitung sowie bereits vorhandene Bewegungserfahrungen. Sie sind Voraussetzungen des Boxers über die Kampfsituation zu reflektieren, die subjektiv verschiedene Abstraktions-, Differenziertheits- und Bewusstseinsgrade aufweisen kann. Umfang und Qualität der Widerspiegelungen dieser Bedingungen haben entscheidenden Einfluss auf das Handlungsergebnis. (vgl. KONZAG; KONZAG, 1980)

\subsection{Maßnahmen zum Management begrenzter kognitiver Infor- mationsverarbeitungskapazität}

Die Untersuchung fortgeschrittener Boxer in komplexen und dynamischen Handlungssituationen zeigt, dass die für Anfänger festgestellten defizitären und suboptimalen Strategien zum Management der begrenzten kognitiven Informationsverarbeitungskapazität erheblich seltener vorkommen. Für den Fall, dass Täuschungssituationen tatsächlich bewusster Verarbeitung bedürfen, ist von Interesse zu wissen, ob die für andere Situationen festgestellten oder postulierten besseren Strategien auch für diesen Spezialfall relevant sind:

Empirische Untersuchungen zum Problemlöseverhalten (DÖNER; KREUZIG, 1983) erbrachten folgende Hinweise zur Ökonomisierung der Kapazitätsnutzung:

1. "Erhöhung des (strukturellen) Wissens verbunden mit

2. einer Präzisierung der Ziele,

3. der Bildung balancierter Ziele und Eingriffsschwerpunkte.

Bezüglich der Planung von Denkschritten dienen der Erhöhung des (strukturellen) Wissens

1. Zustands- und Trendanalysen sowie die

2. Suche nach Indikatoren zur Behebung transparenter Anteile in einer Situation". (FUHRER, 1984)

HACKER (1978) nennt

1. Aktivierung der Wahrnehmung,

2. psychologische Automatisierung bestimmter Aktivitäten (kapazitive Entlastung höherer Regulationsebenen), 
3. Komplexbildung mittels Sprache und

4. antizipative Regulation des Handelns nach intellektueller Durchdringung einer Aufgabe. (vgl. PÖHLMANN; ENDERS, 1983)

Nicht am Ende trägt die hierarchisch-sequentielle Handlungsorganisation außerdem bei, die bewusste Steuerung des Handelns zu ökonomisieren. (vgl. FUHRER, 1984)

KAMINSKI (1982) schlägt vor, Komplexität zu reduzieren durch

1. Einengung des Orientierungskontextes und

2. den Vollzug nur allernotwendigster Handlungen.

Die Maßnahmen sind zweifellos auch in Täuschungssituationen Voraussetzung für erfolgreiches Handeln bei allen Beteiligten. Sie müssen allerdings vor Beginn der eigentlichen Handlungssituation abgeschlossen und als unter Zeitdruck handhabbare Strategie formuliert sein. (vgl. DÖRNER; KREUZIG, 1983)

\subsection{Aufmerksamkeit im Boxen}

Die Einengung des Orientierungskontextes und Aktivierung der Wahrnehmung und die gezielte Konzentration oder Distribution der Aufmerksamkeit auf (vermutete) handlungsrelevante Hinweisreize soll die Menge der eingehenden Informationen verringern und das Bewusstsein nur so viel wie nötig und so wenig wie möglich belasten. (vgl. HACKER, 1978; KAMINSKI, 1982; MAXEINER; 1991) Es ist bekannt, dass auf Wissen und Erfahrung beruhende Erwartungen die Verteilung der Aufmerksamkeit lenken und die Sensibilität für bestimmte Wahrnehmungsinhalte erhöhen. (vgl. SANDERS, 1979; ALLPORT, 1980; BARTH; KIRCHGÄSSNER, 1984)

KONZAG (1981) legte eine sehr wichtige Untersuchung zu Aufmerksamkeitsdistributions-, Konzentrations- und Umschaltfähigkeit im Boxsport vor. Vor allem hinaus wurde noch ein normierter Test zu Distributions- und Konzentrationsfähigkeit bei einfachen und kombinierten Finten durchgeführt. Das Ergebnis war, dass die Zielhandlung selbst die größte Konzentrationsleistung verlangte (bei leichten Variationen zwischen den Kämpfern). Schon frühere Untersuchungen 
hatten gezeigt, dass durch die zusätzliche Beachtung eines Gegners die Trefferzahlen wesentlich zurückgingen, ohne dass eine direkte Behinderung des Boxers erfolgt wäre. (vgl. KONZAG, 1981)

Entsprechende Aufmerksamkeitsverteilung in Täuschungssituationen ist nicht nur für den Täuschenden sondern auch für den zu Täuschenden von größter Wichtigkeit. Die Täuschungshandlungen Bluff verlangt dabei von den Beteiligten Konzentration bei der Bewertung der erhaltenen Information relativ zur eigenen geplanten Strategie, aber nicht gezielte Aufmerksamkeitsverteilung im oben genannten Sinne.

Täuschungsaufstellung und Finte verlangen besonders von den potentiellen Opfern eine Erfolg versprechende Aufmerksamkeitsverteilung. Auf Unwissenheit oder Unvorsicht beruhende Fehler hierbei können die Wahl eines ungeeigneten Bezugssystems für die Wahrnehmung bedeuten und so verhindern, dass aus der erhaltenen Information die richtigen handlungsrelevanten Hinweise gewonnen werden können. Je größer der Zeitdruck dabei ist, desto schwieriger wird diese Aufgabe. Der Täuschende hat es hierbei leichter, da er den Start und den zeitlichen Verlauf der Handlungssituation stärker bestimmen kann. Eine weitere Konzentrationsleistung stellt das bewusste Registrieren von Handlungswiederholungen und -besonderheiten dar, was für die Beteiligten eine bessere Strategiewahl ermöglichen hilft. Konzentrierte Aufmerksamkeit ist somit in Täuschungssituationen eine unerlässliche Voraussetzung für erfolgreiches Handeln. Deshalb werden jedoch die inhaltlichen Probleme der Bewertung der erhaltenen Information am Ort der Aufmerksamkeitszuwendung nicht gelöst.

\subsection{Antizipation im Boxen}

Die Antizipationsfähigkeit des Menschen ist eine Leistungsvoraussetzung, deren Wesen in einem Vorausnahmemechanismus mit Wahrscheinlichkeitscharakter besteht. Antizipation ist die Vorausnahme von Bedingungen, Situationen, Ereignissen, Handlungsverläufen, -ketten, -ergebnissen und Handlungskonsequenzen. Diese Vorausnahme ist immer zielgerichtet und verfolgt eine Absicht 
bzw. einen Zweck. Die Antizipation ist stark vom Erfahrungsschatz einer Person bestimmt. (vgl. KRÜGER, 1982b; LEIST; LOIBL, 1983)

Folgende Merkmale der Antizipation lassen sich auflisten:

- Antizipation ist ein psychischer, kognitiver Vorgang, der vor der Ausführung willkürlicher Bewegungen auftritt. (vgl. FETZ, 1980)

- Antizipation findet man dort, wo sich der Mensch oder ein relevantes Objekt, oder auch beide, in meist schneller Bewegung befinden und ein Sichtreffen oder auch dessen Vermeidung notwendig sind; also vorwiegend im Boxsport. (vgl. RÜSSEL, 1976)

- Auf intellektueller Regulationsebene erklärt Antizipation gedankliche Vorwegnahme künftiger Ereignisse und Handlungen anderer, (vgl. HOBUSCH, 1981) sowie ideell verlaufende Widerspiegelungen eigener Handlungen (HACKER, 1978). Sie ist in diesem Sinn Planungsvariante des Handelns. Durch diese Art der Antizipation werden Erwartungen aufgebaut, die dann die Aufmerksamkeit auf bestimmte Wahrnehmungsinhalte lenken.

- Antizipation als Koordinationsleistung auf sensumotorischer Regulationsebene, die so genannte antizipierende Reaktion, ist dann gegeben, wenn das handelnde Subjekt den Reaktionsprozess durch das Freisetzen des Reaktionsimpulses so früh einleitet, dass die vereinbarte Antwortbewegung mit dem Signalpunkt koinzidiert. (vgl. OBERSTE, 1979)

- Antizipation beinhaltet mehrere verschiedene Richtungen:

1. Vorausnahme des zu erreichenden Ziels (Zielantizipation) (vgl. MEINEL; SCHNABEL, 1977)

2. Vorausnahme der hiervon abhängenden/hierdurch bedingten Handlungen (Programmantizipation) (vgl. MEINEL; SCHNABEL,1977)

3. Vorausnahme der personenspezifischen körpereignen Bedingungen (Wirkungsantizipation) (vgl. WRISBERG; RAGSDALE, 1979)

4. Vorausnahme größerer Zusammenhänge/Handlungsgefüge (z. B. Kampfsituationen). (vgl. FETZ, 1980) 
Die Täuschung und Antizipation sind Antagonisten:

Die Täuschung ist eine taktische Maßnahme, durch die man den Gegner bewusst zu einer falschen Antizipation verleitet. (vgl. HESS, 1982)

Im Boxsport ist die Antizipation im Bereich des Fintierens von besonderer Bedeutung, da durch die Vorausnahme einer Kampfsituation der Boxer seine Vorteile für einen auf die Finte folgenden Angriff besser ausnutzen kann. Daher ist es wichtig, dass im Boxtraining darauf geachtet wird, die Antizipation in allen Bereichen forciert zu trainieren, um höchstmögliche Erfolge zu erreichen. (vgl. KRÜGER, 1982a)

\subsection{Wahrnehmung in Täuschungssituationen im Boxen}

Wahrnehmung ist ein sinnvoller Einsatz in der jeweils gegebenen Situation während des Wettkampfes. Dieser Einsatz setzt eine schöpferische Denktätigkeit voraus, die auf Grund von gespeichertem Wahrnehmungs- und Analysevermögen aus verschiedenen Entscheidungsalternativen die jeweils richtige Lösung herausfiltert. Dabei soll Instinkt, Vernunft sowie Realisierbarkeit der jeweiligen Entscheidung die Grundlage jedes Handlungsplanes sein. Es wird darauf hingewiesen, dass eine taktische Entscheidung von der technischen Güte der Ausführung abhängig ist. Schöpferische Denkfähigkeit und individuelle Kreativität sind für die situative Anwendung und Umsetzung von Angriffs- und Abwehrtaktiken im Boxen erforderlich. (vgl. SCHÖNBORN, 1990)

Reize und Informationen nimmt der Mensch bewusst und unbewusst mit Hilfe seiner Sinnesorgane wahr. Im Boxsport unterscheidet man zwischen optischen, akustischen und taktilen Informationen, die der Boxer in seinem Kurz- oder Langzeitgedächtnis abspeichert. Zu einer optimalen Informationsaufnahme und -verarbeitung benötigt man immer ein Zusammenspiel mehrerer Analysatoren sowie Speichersystemen im Gehirn. So ist das Gelingen boxsportlicher Bewegung nur aufgrund dieser Gleichzeitigkeit möglich. (vgl. GROSSER; HERMANN, 1987) 
Um die Frage zu beantworten, ob und wenn ja wie Wahrnehmungsinhalte in Täuschungssituationen und bei hohem Zeitdruck handlungsrelevante Information liefern können, sollen einige wahrnehmungstheoretische Ansätze auf Hinweise zur Lösung dieses Problems hin betrachtet werden.

Neuere Forschungen befassen sich mit der Erforschung konkreter und inzwischen auch sehr komplexer Phänomene, ohne gleich den Anspruch zu erheben, eine alle Wahrnehmungsprobleme umfassende Theorie zu bilden. Die Forschungsergebnisse kommen dabei aus den Bereichen

- Neurophysiologie,

- experimentelle Psychologie (und Philosophie) sowie

- Computerwissenschaften.

Für unser Problem der Mustererkennung sind dabei neurophysiologische Ergebnisse aus Untersuchungen an einzelnen sensorischen Nervenzellen von Interesse. (vgl. ALBRECHT, 1982; ROCK, 1985)

Die Erfindung dieser Merkmalsdetektoren beim Menschen erfolgte ca. um 1960. Auf der Basis dieser Erfindung entwickelte sich eine Theorie der Merkmalsdetektoren, deren Verfechter glaubten, Wahrnehmungsphänomene ausschließlich mit Hilfe neuronaler Mechanismen erklären zu können. (vgl. ROCK, 1985)

Alle denkbaren Bewegungen im Boxen können als Objekte bezeichnet werden. Diese Objekte lassen sich in (zwei oder) mehrere Klassen kategorisieren. Die Mustererkennung hat dann die Aufgabe, die Klassenzugehörigkeit eines gegebenen Objektes zu bestimmen. Gehört die vorliegende Bewegungshandlung in die Klasse der Finten oder in die der durch sie angetäuschten tatsächlichen Zielhandlung? Mustererkennung bezieht sich dabei nicht nur auf einzelne Bewegungen sondern auch auf Handlungssequenzen, wie sie z. B. für eine Täuschungsaufstellung charakteristisch sind. Jede Musterklasse ist repräsentiert durch einen die zentrale Tendenz des Musters widerspiegelnden (idealen) Prototyp, der die qualitativ invarianten Charakteristika beinhaltet. (vgl. BREMERMANN, 1976; LEIST; LOIBL, u. a., 1983) 
Die Bewegungswahrnehmung erfolgt über perzeptive Komponentenzerlegung. Der Wahrnehmungsfluss wird dabei in Invarianten besserer Ordnung, wie z. B. Geschwindigkeitsvektoren, zerlegt. Beachtet werden Relativbewegungen zu einem Bezugssystem bzw. Relativbewegungen der einzelnen Bezugssysteme untereinander. (vgl. REED, 1978; LEIST; LOIBL, u. a., 1983; WARREN; SHAW, 1985) Die Ansätze von JOHANSSON Theorie der Bezugssystembildung (1978) und GIBSON (ökologischer Ansatz 1979) sowie die Arbeiten von LEE (1978; 1980) und NOGUCHI; HAISHI u. a., (1994) folgen den oben genannten Ausführungen.

Bei weiterer Untersuchung im neurophysiologischen Bereich zum Problem der Merkmalsdetektoren gibt es einen Bezug zwischen den durch Komponentenzerlegung erhaltenen Invarianten aus Sicht der Bewegungs- und Wahrnehmungstheorie und den aus Merkmalsdetektoren abgebildeten Reizmustern. Ist das invariante Merkmal zur Unterscheidung einer Finte und der hierdurch vorgetäuschten Handlung, z. B. der Beschleunigungsverlauf des Körperschwerpunktes, und hat die durch die Merkmalsdetektoren übermittelte Reizsequenz längst eine Bedeutungsgebung erfahren (Finte oder nicht Finte), dann kann Zeit bei der Wahrnehmung durch Interpolation gespart werden. Eine Änderung des Beschleunigungsverhaltens könnte so bereits nach kürzester Zeit erfahren und bewertet werden, noch bevor die Änderung bewusst registriert wird. (vgl. LEIST; LOIBL u. a., 1983; WARREN; SHAW, 1985)

\subsection{Ausführungs- und Kontroll- Regulation im Boxkampf}

Bei der Ausführungsregulation interessiert besonders inwieweit bei Finte und Täuschungsaufstellung Handlungssequenzen als solide Kombinationen mechanisiert oder variabel in Abhängigkeit vom gegnerischen Verhalten eingesetzt werden können. Wird der Täuschende durch gegnerisches Verhalten überrascht, gelten für inn dieselben Fragen, die zum Verhalten des möglicherweise Getäuschten zu stellen sind. Dabei interessiert vor allem, ob das Wissen um die Möglichkeit, getäuscht zu werden, dazu führt, dass für den Fall ein Alternativprogramm automatisiert ist, das dann, unter bestimmten physiologischen und 
physikalischen Bedingungen, sofort angeschlossen werden kann. Des Weiteren soll erklärt werden, wie sich das Hereinfallen auf eine Finte auswirkt und welche Vorstellungen über die Organisation der Ausführungsregulation im Anschluss an die bisherigen Überlegungen den größten Erklärungswert haben.

Ausführungs- und Kontrollregulation umfassen diejenigen psychischen Komponenten, die nach der Handlungsziel- und Handlungsprogrammentscheidung die eigentliche Durchführung der Handlung, deren Kontrolle und Auswertung garantieren. Es handelt sich dabei insbesondere um Prozesse der Steuerung des aktuellen Handlungsvollzugs einschließlich der reafferenten Verlaufskontrolle (Bewegungslenkende und regulative Reafferenz). Anforderungen an die Ausführungsregulation ergeben sich aus der

- Vielfalt der möglichen Handlungsprogramme,

- Variabilität der Handlungsausführung,

- Geschwindigkeit der Handlungsausführung,

- Genauigkeit der Handlungsausführung,

- Handlungsausführung bei direkter und indirekter gegnerischer Bedrängnis,

- Handlungsausführung unter hoher psychischer und physischer Belastung. (vgl. KONZAG, 1981)

\subsubsection{Auswirkung gelungener Finten und Täuschungsaufstel- lung}

Nicht nur im Fall der Finte sondern auch in dem der Täuschungsaufstellung beruht das Gelingen der Täuschungshandlungen auf Fehlern in der Orientierungs- und Entscheidungsregulation des Gegners. Der Erfolg beruht in beiden Fällen auf der Wahl einer falschen Antworthandlung, die in ihren räumlichen und vergänglichen Auswirkungen unterschiedliche Konsequenzen hat.

Der Effekt einer gelungenen Finte ist es, dass der Getäuschte sich in eine der tatsächlichen zu verteidigenden Bewegung entgegengesetzter Richtung zu bewegen beginnt. Während oder nach Beendigung seiner Bewegung realisiert er den Fehler und will sofort das begonnene Bewegungsprogramm stoppen oder ein Neues anschließen, um den Gegner doch noch am Durchsetzen seiner Ab- 
sicht zu hindern. Unter welchen Bedingungen dies möglich ist, hängt von mehreren Faktoren ab:

1. Im Fall der Finte ist zu beachten, dass bei der sukzessiven Darbietung der Reize das zweite Signal das erste widerruft. Angenommen, die erste Reaktion kann nicht mehr verhindert werden, dann gilt für die Reaktionszeiten, die obschon sie als Unterbrechungssignal erfolgen, dass - entgegen der Erwartung - dafür weniger Zeit benötigt wird als für eine ungestörte Reaktionszeit. Diese Verkürzung ist am größten bei einem vorauseilendem Unterbrechungssignal wie bei der Finte: die Verkürzung beträgt $\geq 100 \mathrm{msec}$ bei einer Reaktionszeit von 400 msec. (vgl. RÜSSEL, 1976)

2. Wenn die Reaktion auf zwei dicht aufeinander folgende Reize verlangt wird, wird die zweite Reaktion verzögert einsetzen. Dieser zeitliche Abstand zwischen den zwei Reaktionen auf die zwei Reize ist dann relativ am längsten, wenn der Abstand zwischen den Reizen möglichst kurz ist. (vgl. KRÜGER, 1982 b) Diese so genannte psychologisch refraktorische Periode wird als zentrales Phänomen (Begrenzung bewusster kognitiver Informationsverarbeitungskapazität) angesehen und mit der Einkanalhypothese belegt. (vgl. RITZDORF, 1982)

\subsubsection{Bewegungsausführung bei Finten und Imitationstäu- schungen im Boxen}

Im Anschluss werden Bewegungsbeschreibungen und Analysen von Finten gegeben. Dafür gibt es mehrere Gründe:

- Täuschungswirkung aus Irritation erzielen häufig schon Bewegungen, die ganz grobe invariante Charakteristika der Zielbewegung aufgreifen, wie z. B. das Vortäuschen eines geraden Schlages mit der Führungshand.

- Täuschungswirkung erzielen bisweilen auch völlig situationsinadäquate Bewegungen, bei denen der Beobachter erst den Sinn zu verstehen versucht und 
dadurch wertvolle Zeit vergeudet. Diesen Irritationseffekt erzielen auch Anfänger gegenüber Fortgeschrittenen durch ihre unorthodoxe Boxweise.

- Eine Täuschungshandlung, die durch Imitation und nicht durch Irritation ihre Wirkung erzielen soll, muss in erster Linie der durch sie vorgetäuschten Handlung so ähnlich wie möglich sein. Da bei Zielbewegungen, bei denen nur funktionale und nicht noch zusätzlich stilistische Gesichtspunkte im Vordergrund stehen, die Bewegungsausführung höchst unterschiedlich ist, muss jeder Täuschende seine Bewegung glaubhaft imitieren.

- Eine gute Imitation beinhaltet dabei nicht nur eine räumliche sondern auch eine zeitliche Annäherung an die Originalbewegung. Eine perfekte Imitation kann deshalb auch nur bei Bewegungen gelingen, bei denen der Beschleunigungsverlauf des Körperschwerpunktes in beiden Fällen identisch sein kann (z. B. die kombinierte Finte mit Rumpf und Beinen). Bei allen Finten aber, bei denen die eigentlich beabsichtigte Bewegung nicht mit der Richtung der Finte übereinstimmt, kann der Beschleunigungsverlauf nur bis zu einem physikalisch determinierten Umkehrpunkt imitiert werden. 


\section{Die Finten im Boxen}

\subsection{Der Angriff unter der Verwendung von Finten}

Der Angriff beinhaltet alle Methoden, die der Boxer in Geschwindigkeit, Vertrauen, Cleverness und mittels Täuschung und der Kraft seines ganzen Körpergewichtes verwendet, um den Gegner in den erlaubten Körperzonen zu treffen und inn im Kampf zu besiegen. Der Angriff soll zunächst von langer Distanz angefangen werden. Der Boxer soll die Perfektion der Verteidigung, des Kunstschlages und die modernen Methoden anwenden und durch Fintieren mit den Armen oder dem Oberkörper oder den Beinen den Gegner an einem ungeschützten Punkt schlagen. Im Erfolgsangriff darf der Boxer sein Gleichgewicht nicht verlieren. Wenn er die Verteidigung durchführt und zur Gegenattacke ansetzt, erzielt er den Erfolgsangriff, indem er seine Fähigkeiten und die des Gegners genau einschätzt.

Als Angriffshandlungen bezeichnet man in der Boxtaktik das zielgerichtete aktive Einwirken auf den Gegner, das durch das Ausführen von Schlägen vorwiegend in der Vorwärtsbewegung gekennzeichnet ist. Der Boxer kann die Angriffshandlungen in allen drei Distanzen durchführen. Entsprechend der unmittelbar vorausgegangenen taktischen Handlungen unterscheiden wir zwischen direktem Angriff (nach Verteidigungshandlungen des Gegners) und Gegenangriff (unmittelbar nach gegnerischem Angriff). Nach neueren Auffassungen gibt es im Angriff vier Phasen: Vorbereitung (unter der Verwendung von Finten), Durchführung, Abschluss, Weiterführung.

Der Boxer versucht bei Angriffen ohne Vorbereitung auf der Grundlage der analysierten Kampfsituation (geistige Vorbereitung) für den Gegner unerwartet entscheidende Treffer anzubringen. Bei der näheren Beschreibung des direkten Angriffs konzentrieren wir uns auf den Angriff mit Vorbereitung. Der direkte Angriff, in der Sportpraxis nur als Angriff bezeichnet, stellt sich im Wettkampf als abgeschlossene Handlung dar. Dabei versucht der Boxer mit den verschiedensten taktischen Mitteln, wie Körperfinten und Schläge, Scheinangriffen und dem so genannten Ziehen, seinem Gegner eine zum Angriff günstige Position zu bieten. Hauptsächlich verwendet der Boxer zur Verteidigung des gegnerischen 
Angriffs Elemente der passiven Verteidigung, indem er die Angriffsschläge des Gegners durch Ausweichbewegungen vermeidet, oder er stoppt den gegnerischen Angriff durch Kontern oder Mitschlagen. Die Beherrschung des Gegenangriffs setzt ein hohes Niveau an technisch-taktischen Fähigkeiten und Fertigkeiten voraus. Er sollte zum Repertoire jedes Spitzenboxers gehören. (vgl. SONNENBERG, 1993; FIEDLER, 1997; ELLWANGER; ELLWANGER, 1998)

\subsection{Die Angriffschläge}

Der beste Angriffsschlag für einen Anfänger ist der gerade Schlag mit dem linken oder rechten Arm gegen den Kopf oder den Oberkörper. Um einen neuen Schlag vorzubereiten, muss der Boxer die Schnelligkeit seines Reaktionsvermögens und seines Handelns unter Belastungsbedingungen perfektionieren. (BASTIAN, 2003) Ein erfahrener Boxer kann seitliche Haken und Aufwärtshaken gegen den Kopf oder den Oberkörper erzielen. Der Boxer kann die Angriffsdurchführung ausführen, indem er sich entsprechend seiner taktischen Absicht durch die vorbereitenden Handlungen in eine günstige Ausgangsposition im Schlagbereich des Gegners gebracht hat. Der Angriff kann in Abhängigkeit von der aktuellen Kampfsituation und den individuellen Voraussetzungen des Boxers als Einzelschlag, Schlagverbindung, Schlagkombination oder Serie erfolgen. Angriffsvorbereitung und Angriffsabschluss bilden eine Einheit. Der Angreifer sollte Vorbereitungshandlungen und die eigentliche Angriffsführung im Verlaufe des Kampfes variieren, um eine mögliche Einstellung des Gegners zu erschweren.

Nach der Angriffsdurchführung muss der Boxer der Angriffsabschluss und die Sicherung des Angriffes ausführen, um dem Gegner keine Treffermöglichkeiten zu geben. Und er muss versuchen, so schnell wie möglich aus dem Schlagbereich des Gegners zu gelangen. Im Wettkampf werden oft beide Formen kombiniert. Dabei muss das Lösen vom Gegner grundsätzlich mit einem Sicherungsschlag verbunden werden. Die für Anfänger geltende Grundregel, den Angriff nach Möglichkeit mit einem geraden Stoß der Führungshand abzu- 
schließen (Drücken des Gegners in die weite Distanz, Einnehmen der normalen Kampfposition), hat ihre volle Gültigkeit.

Der Boxer kann sich auch nach erfolgtem Angriff in einer erfolgsversprechenden Kampfposition befinden, z. B. nach erzielten Wirkungstreffern. Oder der Gegner steht am Seil, bzw. ein erfolgreicher Abschluss wird durch den Gegner verhindert, so wird der Angriff weitergeführt. Außerdem bedient sich der Boxer der gleichen Mittel wie bei der Angriffsführung. Benutzt der Boxer den ersten Angriff bewusst, um für die Weiterführung günstige Kampfsituationen zu schaffen, so werden auch die Begriffe Angriff in erster Absicht und Angriff in zweiter Absicht verwendet. Sehr geeignet und zweckmäßig ist die Angriffsweiterführung mit Schlagserien, nach der der Gegner durch den Angriff ans Seil oder in die Ringecke manövriert wurde. (vgl. SONNENBERG, 1993; WEISE, 1995; FIEDLER, 1997)

\subsection{Das Fintieren}

Die Fertigkeit des Fintierens setzt eine nur allmählich zu erlernende Erfahrung voraus. Wenn der Boxer die oben erwähnten Fähigkeiten wie Reaktionsvermögen, Cleverness beherrscht, kann er durch Fintieren am Ende erfolgreich siegen. Boxer und Gegner müssen Beine, Oberkörper und Kopf nutzen, um beim jeweiligen Gegner Finten anzubringen. Beim Fintieren täuscht man den Gegner dadurch, dass man mit einer Kombination aus Armen, Beinen und Kopf oder unter Anwendung nur eines dieser Körperteile auf einen ungeschützten Körperteil des Gegners zielt, der Gegner reagiert mit Verteidigung des jeweiligen Körperteils. Daraufhin greift der Boxer ein anderes, vom Gegner nicht erwartetes Körperteil an.

Die Effektivität eines Angriffs ist hochgradig von der Eigenschaft der vorbereitenden Handlungen abhängig. Sie sind in der Konsequenz darauf gerichtet, eine möglichst günstige Situation für die Angriffsdurchführung zu erarbeiten und zielen auf die Ausforschung (Erkundung), Täuschung und das Manövrieren des Gegners ab. Der Ausprägungsgrad der Fähigkeit, die beabsichtigten taktischen Verhaltensweisen und seine Stärken und Schwächen möglichst schnell zu er- 
kennen sowie ihm über die eigene Kampfweise möglichst wenig Informationen zukommen zu lassen, ist für den gesamten Kampfverlauf von entscheidender Bedeutung. Die vorbereitenden Handlungen unterteilen wir in Finten, Täuschungsaufstellung, Scheinhandlungen und Manöver.

Für die Taktikausbildung eines Boxanfängers ist es bedeutend, Standardsituationen zu schaffen und diese ständig zu erweitern. Bereits bei Partnerübungen lernt der Boxer, dass Einzelstöße einmalig Treffer bringen. Also müssen Aktionen gestartet, herausgearbeitet, in das Training eingeplant und dann geübt werden, die nicht voraussehbar und berechenbar sind. Dazu gehört zum Beispiel das Andeuten eines Stoßes, der gar nicht ausgeführt wird, aber den Gegner veranlasst, eine Abwehrbewegung einzuleiten. Derartige Aktionen nennt man Finten.

Beispiel: Die linke Führungshand wird als Schlag angedeutet, die Schulter nach vorn gebracht und der Arm nur zur Hälfte gestreckt. Das ist ein idealer Auftakt für die dann folgende rechte Gerade, deren Drehimpuls durch das ruckartige Zurückziehen der linken Hand verstärkt wird.

- Antäuschen von Stößen der Führungshand zum Kopf und Stoß der Schlaghand zum Körper;

- Antäuschen von Stößen der Führungshand zum Körper und Stoß der Schlaghand zum Kopf;

- Antäuschen eines Stoßes der Schlaghand zum Kopf und Stoß der Führungshand zum Kopf mit Schritt nach vorn.

Mit anderen Techniken kann erst begonnen werden, nachdem die geraden Stöße zumindest in ihrer Grobform beherrscht sind. Wir sollten dabei nicht vergessen, dass die Zeit, die für eine Boxaktion zur Verfügung steht, nur begrenzt ist: Je mehr ein Sportler kann, desto schneller sind seine Aktionen und desto weniger Zeit hat der sportliche Gegner, sich auf diese Aktionen einzustellen. Die Gesamtzeit für eine linke Gerade ist kürzer als eine Sekunde. Daraus ergibt sich eine derart kurze Reaktionszeit, dass man versteht, warum Mitdenken, Vorausdenken und Voraushandeln eine Grundvoraussetzung für das Boxen ist. Es ist stets derjenige im Vorteil, der mit einer Aktion beginnt. Deshalb werden auch zuerst die geraden Stöße gelehrt, bei denen die Hand länger unterwegs 
ist. Stöße in der Halbdistanz und im Nahkampf werden erst später gelehrt, da für ihre Erfassung und Abwehr viel weniger Zeit zur Verfügung steht. (vgl. FIEDLER, 1976; MÜLLER, 1991; SONNENBERG, 1993;)

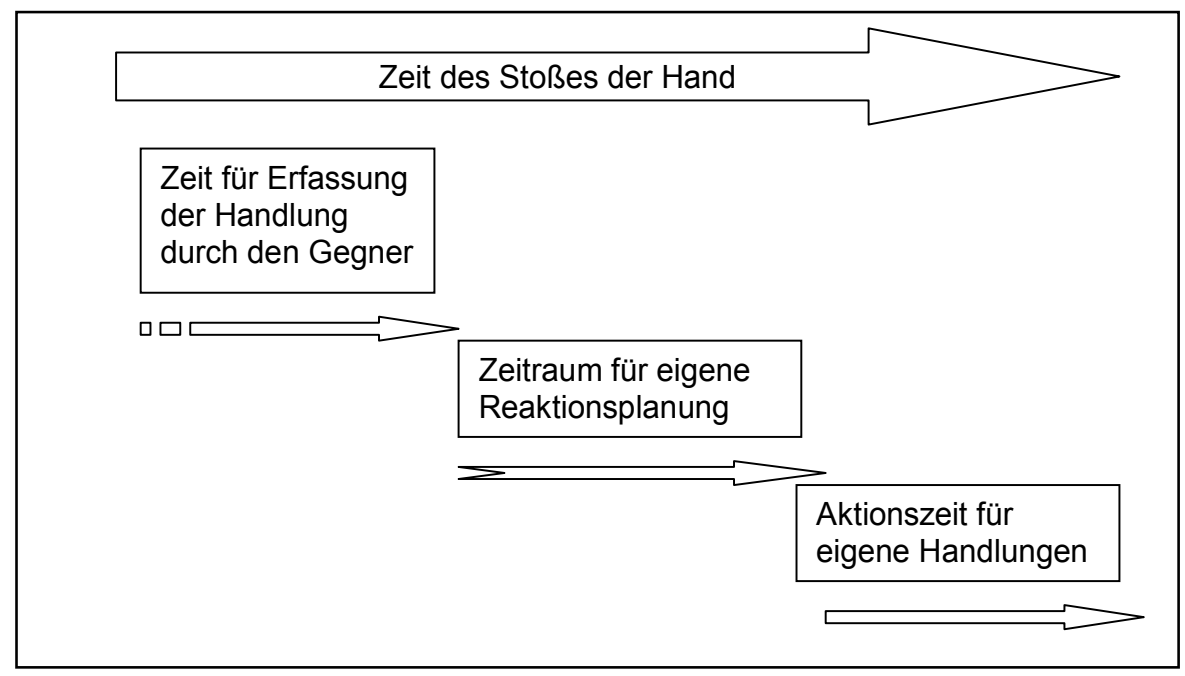

Abb. 34: Zeitlicher Ablauf von Schlag und Reaktion (modifiziert nach: ELLWANGER; ELLWANGER, 1998)

\subsection{Arten der Finten im Boxen}

\section{Einfache Finten}

\section{A: Mit den Augen}

Die Blickrichtung täuscht ein anderes als das tatsächliche Angriffziel vor, damit der getäuschte Gegner den falschen Körperbereich deckt, um inn an einer ungedeckten Stelle zu treffen.

\section{B: Mit den Beinen}

Diese Fintenart verwenden nach meinen Studien und Erfahrungen größere Boxer häufiger als kleinere Boxer, wobei hier der Größenunterschied in den jeweiligen Gewichtsklassen $10-15 \mathrm{~cm}$ im Maximum beträgt. Der große Boxer boxt häufiger aus der Weitdistanz. Kleinere Boxer verwenden diese Fintenart, um in die Halb- oder Nahdistanz zu kommen bzw. wenn kleine Boxer gegeneinander 
antreten. Es gibt drei Fintenarten mit den Beinen, die der Boxer verwenden kann:

1. Abtauchen: Der Boxer macht schnell eine leichte Kniebeuge und behält die Armstellung bei. Nach dieser Finte kann der Boxer einen Schlag auf den Oberkörper oder auf den Kopf platzieren.

2. Halber Vorwärtsschritt: Aus der Boxstellung macht der Boxer mit dem vorderen Fuß einen halben Schritt (etwa 10 bis $20 \mathrm{~cm}$ ) vorwärts und geht so schnell wie möglich zurück in die ursprüngliche Boxstellung.

3. Halber Rückwärtsschritt: Aus der Boxstellung macht der Boxer mit dem hinteren Fuß einen halben Schritt (etwa 10 bis $20 \mathrm{~cm}$ ) rückwärts und geht dann so schnell wie möglich zurück in die ursprüngliche Boxstellung.

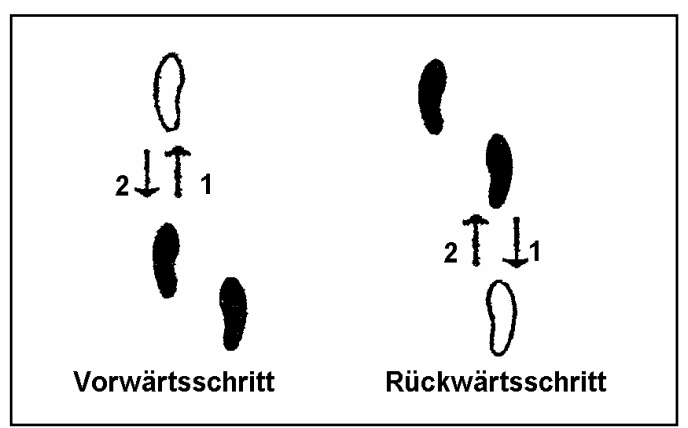

Abb. 35: Finten mit halbem Vorwärtsschritt und halbem Rückwärtsschritt (eigene Darstellung)

\section{C: Mit dem Rumpf}

Diese Fintenart verwenden nach meinen Studien und Erfahrungen kleinere Boxer häufiger als größere Boxer, wobei hier der Größenunterschied in den jeweiligen Gewichtsklassen $10-15 \mathrm{~cm}$ im Maximum beträgt. Der kleinere Boxer boxt häufiger aus der Nah- und Halbdistanz. Es gibt drei Fintenarten mit dem Rumpf, die der Boxer verwenden kann:

1. Vorwärts ausweichen: Der Boxer neigt den Rumpf schnell leicht nach vorn und geht ganz schnell zurück in die Boxstellung. 
2. Vorwärts ausweichen links: Der Boxer macht schnell eine leichte Neigung des Rumpfes nach vorne links und geht ganz schnell zurück in die Boxstellung.

3. Vorwärts ausweichen rechts: Der Boxer macht schnell eine leichte Neigung des Rumpfes nach vorne rechts und geht ganz schnell zurück in die Boxstellung.

\section{D: Mit dem Armen}

Das Fintieren mit den Armen gehört zu den wichtigsten Methoden, die jeder Anfänger lernen und beherrschen muss, da er sich hiermit die Fähigkeit aneignet, nicht aus der Nahdistanz oder Halbdistanz anzugreifen zu müssen, sondern bereits aus der Weitdistanz mit Vertrauen und Selbstbewusstsein fintieren kann. Auch wenn er nicht erfolgreich fintiert, wird er zumindest nicht vom Gegner geschlagen. Kleine und große Boxer verwenden diese Fintenart.

1. Kreisbewegung Fäustling nach innen und außen: Aus der Anfangsposition führt der Boxer mit dem vorderen Fäustling eine Kreisbewegung nach innen oder außen aus, dabei soll der Kreisdurchmesser $10 \mathrm{~cm}$ sein, und die Bewegung ganz schnell aus der Schulter kommen. Der Boxer muss die Kreisanzahl und Kreisrichtung wechseln, danach kann der Boxer Angriffsschläge machen, aber, wenn er nach dieser Finte einen seitlichen oder Aufwärtshaken schlagen möchte, sollte er einen Vorwärtsschritt machen, um auf mittlere Distanz oder kurze Distanz zu kommen.

2. Kurzer gerader Schlag: Für diese Fertigkeit wird immer die Führungshand verwendet, weil sie näher am Gegenkämpfer ist. Das Ziel ist es, den Gegner zum Öffnen einer Bresche oder zu einer Verteidigungshandlung zu bewegen, um dann einen Schlag oder Serienschläge auf die freie Zone zu machen.

3. Bewegung Schulter nach vorn: Aus der offensiven Position macht der Boxer mit einer Schulter eine ganz schnelle Bewegung nach vorn, um den Gegner zu irritieren. Der Gegner kann den folgenden Angriff somit nicht einschätzen. 


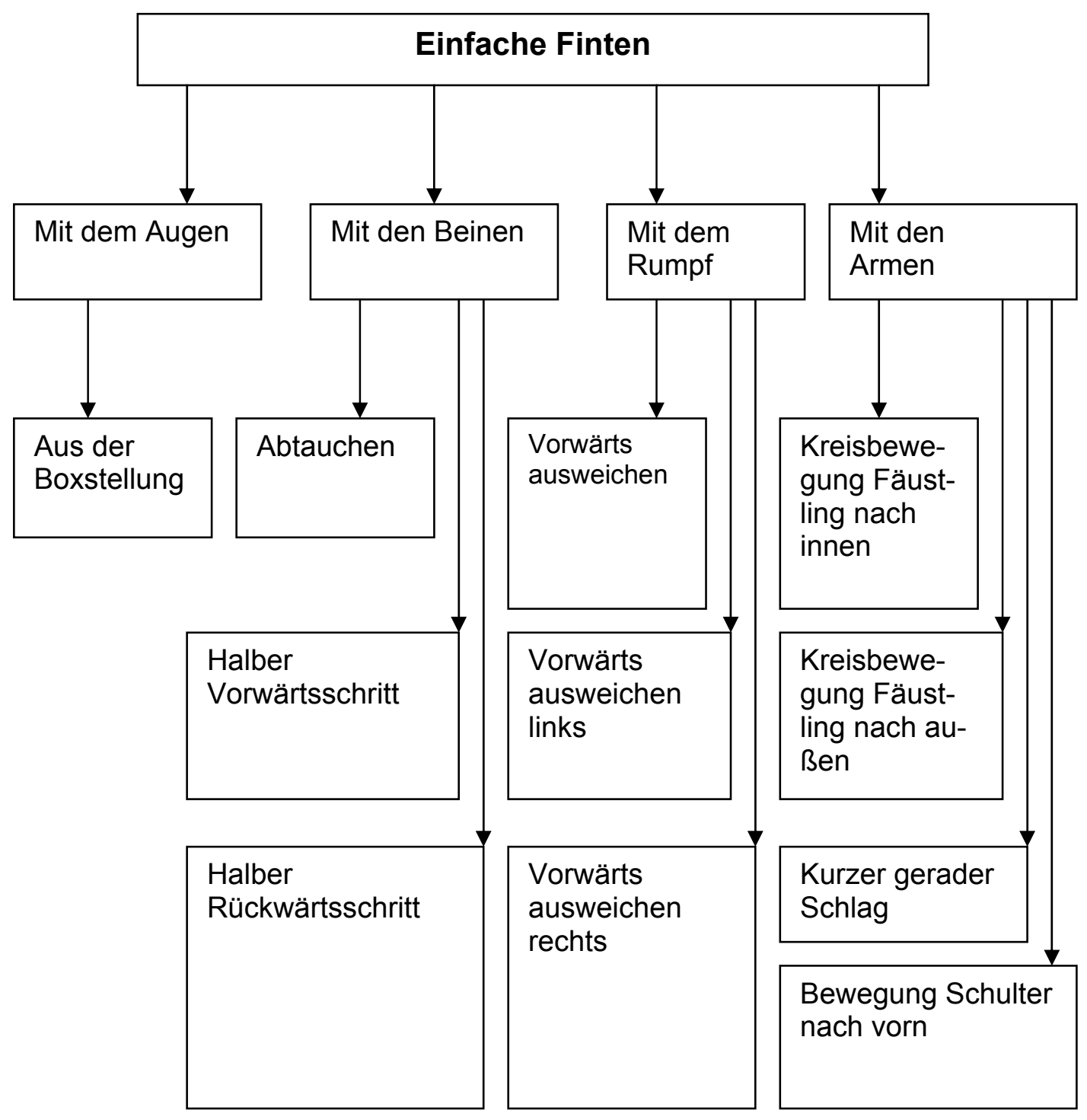

Abb. 36: Klassifizierung der einfachen Finten (eigene Darstellung)

\section{Kombinierte Finten}

Die kombinierte Finte schließt bei allen in Abb. 37 dargestellten Kombinationen den Einsatz des Fintierens mit den Augen ein, weil sich in jeder der dargestellten Kombinationen die Möglichkeit ergeben kann, auch mit den Augen zu arbeiten. 


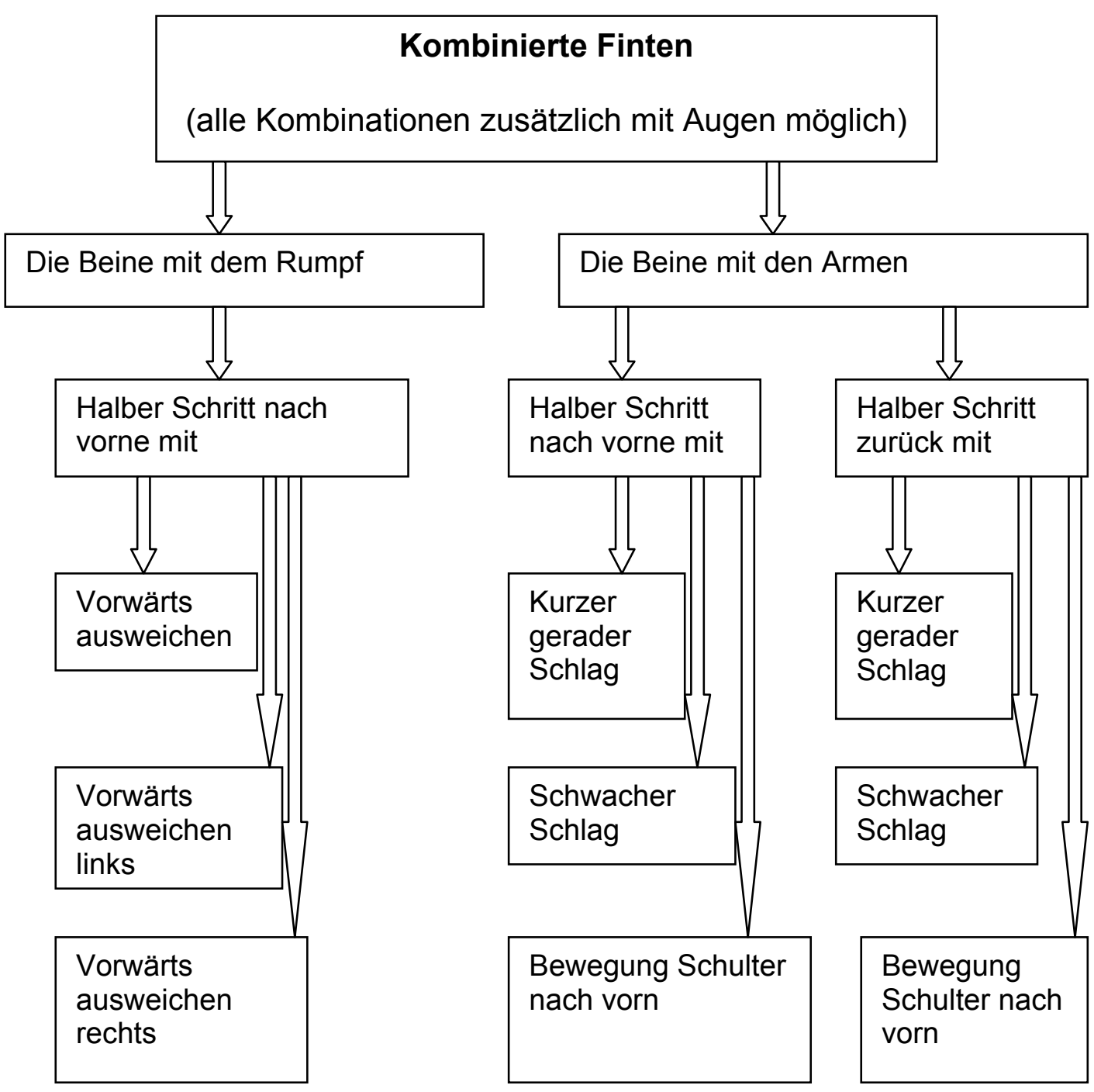

Abb. 37: Klassifizierung der kombinierten Finten (eigene Darstellung)

\section{A. Die Beine mit dem Rumpf}

1. Halber Schritt nach vorne und vorwärts ausweichen: Der Boxer macht mit dem vorderen Fuß schnell einen halben Schritt (etwa 10 bis $20 \mathrm{~cm}$ ) vorwärts, neigt den Rumpf schnell leicht vorwärts und geht sofort zurück in die Boxstellung.

2. Halber Schritt nach vorne mit vorwärts ausweichen links: Diese Fintenart entspricht der vorherigen Finte. Der Boxer neigt den Rumpf dabei schnell leicht nach vorne links. 
3. Halber Schritt nach vorne mit vorwärts ausweichen rechts: Diese Fintenart entspricht den vorherigen Finten. Der Boxer macht eine leichte Rumpfneigung nach vorne rechts.

\section{B. Die Beine mit den Armen}

1. Halber Schritt nach vorne mit kurzem geradem Schlag.

2. Halber Schritt nach vorne mit schwachem Schlag.

3. Halber Schritt nach vorne mit Schulterbewegung nach vorn.

4. Halber Schritt zurück mit kurzem geradem Schlag.

5. Halber Schritt zurück mit schwachem Schlag.

6. Halber Schritt zurück mit Schulterbewegung nach vorn. 


\section{Untersuchungsmethode}

\subsection{Auswahl des Stichprobe}

An den Boxwettkämpfen bei den Olympischen Spielen im Sydney 2000 nahmen 310 Boxer aus 75 Ländern teil. (siehe Anhang 3)

Im Halbfinale (22 Länder) standen 48 Boxer, nämlich 7 Boxer aus (RUS), 6 Boxer aus (CUB), 5 Boxer aus (UKR), 4 Boxer aus (KAZ), 4 Boxer aus (USA), 3 Boxer aus (UZB), je 2 Boxer aus (FRA), (THA) und (ROM), je 1 Boxer aus (ESP), (CSE), (GBR), (PRK), (MAR), (MEX), (ALG), (MDA), (AZE), (HUN), (GER), (GEO) und (ITA). (siehe Anhang 4)

Im Finale (12 Länder) standen 24 Boxer, nämlich 5 Boxer aus (RUS), 4 Boxer aus (CUB), 4 Boxer aus (KAZ), 2 Boxer aus (USA), 2 Boxer aus (UKR), und je 1 Boxer aus (FRA), (ESP), (THA), (UZB), (ROM), (CSE) und (GBR). (siehe Anhang 5) Die analysierten Kämpfe sind in Anhang 6. Es wird hierbei deutlich, dass sich die nicht aufgezeichneten Kämpfe gleichmäßig auf die leichten und schweren Gewichtsklassen verteilen. Wenn zur Bestimmung der Unterschiede zwischen den leichten und den schweren Gewichtsklassen einzelne Gewichtsklassen aus der Rechnung herausgenommen wurden, wurden somit Werte aus der Rechnung eliminiert, die sowohl durch realen Rechenwerte als auch durch nicht vorhandene Werte gekennzeichnet waren.

In der Arbeit habe ich die Kämpfe von den Fernsehsendern Eurosport, ZDF und WDR aufgenommen, aber leider wurden nicht alle Halbfinalkämpfe übertragen; danach haben wir versucht, die fehlenden Kämpfe zu bekommen, und zwar vom Olympic Museum Lausanne und vom Deutschen Amateur-Box-Verband. Aber auch das blieb ohne Ergebnis. (siehe Anhang 1) Deshalb habe ich in der Arbeit 16 Halbfinal- und 12 Finalkämpfe der Olympischen Spiele in Sydney analysiert. Die Anzahl reicht aus, um im Hinblick auf Fintierverhalten und die entsprechenden Boxschulen eine Aussage zu treffen. 


\subsection{Konzeption und Auswertung der Kämpfe}

Ich habe ein Analysesystem aufgebaut, das 19 verschiedene Arten von Finten beim Boxen unterscheidet. Danach habe ich ein spezielles Analysesystem für die Fintenstile für jeden Kampf aufgebaut, um die Analyse zu erleichtern (siehe Anhang 7). Es besteht aus einfachen Finten (mit den Beinen, mit dem Rumpf, mit den Armen) und kombinierten Finten mit den Beinen und dem Rumpf und den Beinen und Armen (Halber Schritt nach vorne, Halber Schritt zurück).

Zuerst habe ich die Kämpfe mit dem Videorecorder von SEG Typ VCR $2350 \mathrm{im}$ Analysesystem analysiert, nachher habe ich die Kämpfe von dem Videokassetten auf den Computer durch die TV-Karte von Typ Hauptauge gespeichert, um die Kämpfe auf dem Computer weiter zu analysieren. Mit Windows DVD Version 5 konnte ich die Kämpfe mit verschiedenen Geschwindigkeiten sehen.

Das Fernsehmaterial eignete sich für die Videoanalyse. Bei den fünf für die Gütekriterien ausgewählten Kämpfen waren die Boxer von der Seite, von Vorn, sowie von Oben gut sichtbar, so dass die Finten gut analysierbar waren.

\subsection{Reliabilität}

Im Hinblick auf die Genauigkeit der Messung ist zum einen die Anzahl der Fernsehkameras, mit denen die Bilder aufgenommen wurden, von Bedeutung. Die Boxkämpfe wurden in der Regel von fünf Kameras aufgenommen, die in den Positionen A, B, C, D und aus der Vogelperspektive (vgl. Abb. 38) standen. Hierdurch war es fast immer möglich, beide Kämpfer in einer Weise auf dem Bildschirm zu sehen, dass die Finten beider gut analysiert werden konnten. Allerdings gab es zwischendurch auch immer wieder Momente, in denen dies nicht der Fall war. Modellhaft wurden diese für die Kämpfe 1) BRAHIM ASLOUM (Frankreich) gegen RAFAEL LOZANO (Spanien) im Halbfliegengewicht, 2) OLEG SAITOV (Russland) gegen SERGEY DOTSENKO (Ukraine) im Weltergewicht sowie 3) HARRISON AUDLEY (Großbritannien) gegen MUKHTARKHAN DILDABEKOV (Kasachstan) im Superschwergewicht analysiert. Es zeigte sich hierbei, dass für 1) 1,561 Sek. (0,325 \% des Kampfes), für 2) 1,386 
Sek. (0,288 \% des Kampfes) und für 3) 1,474 Sek. (0,307 \% des Kampfes) die Kämpfer sich nicht in einer Position befanden, die eine lückenlose Analyse zuließen. Bei durchschnittlich 0,307 \% der Zeit ohne richtige Bilder für die Analyse der Finten konnten die Daten als sehr reliabel bezeichnet werden. (vgl. KRÜGER, 1985)

Bei Zweikampfsportarten ist die Test-Retest-Reliabilität nicht sinnvoll einsetzbar, da ein Kämpfer immer nur so kämpfen kann, wie es der jeweilige Gegner in der jeweiligen Situation zulässt. (BALLREICH, 1970, 35f.)

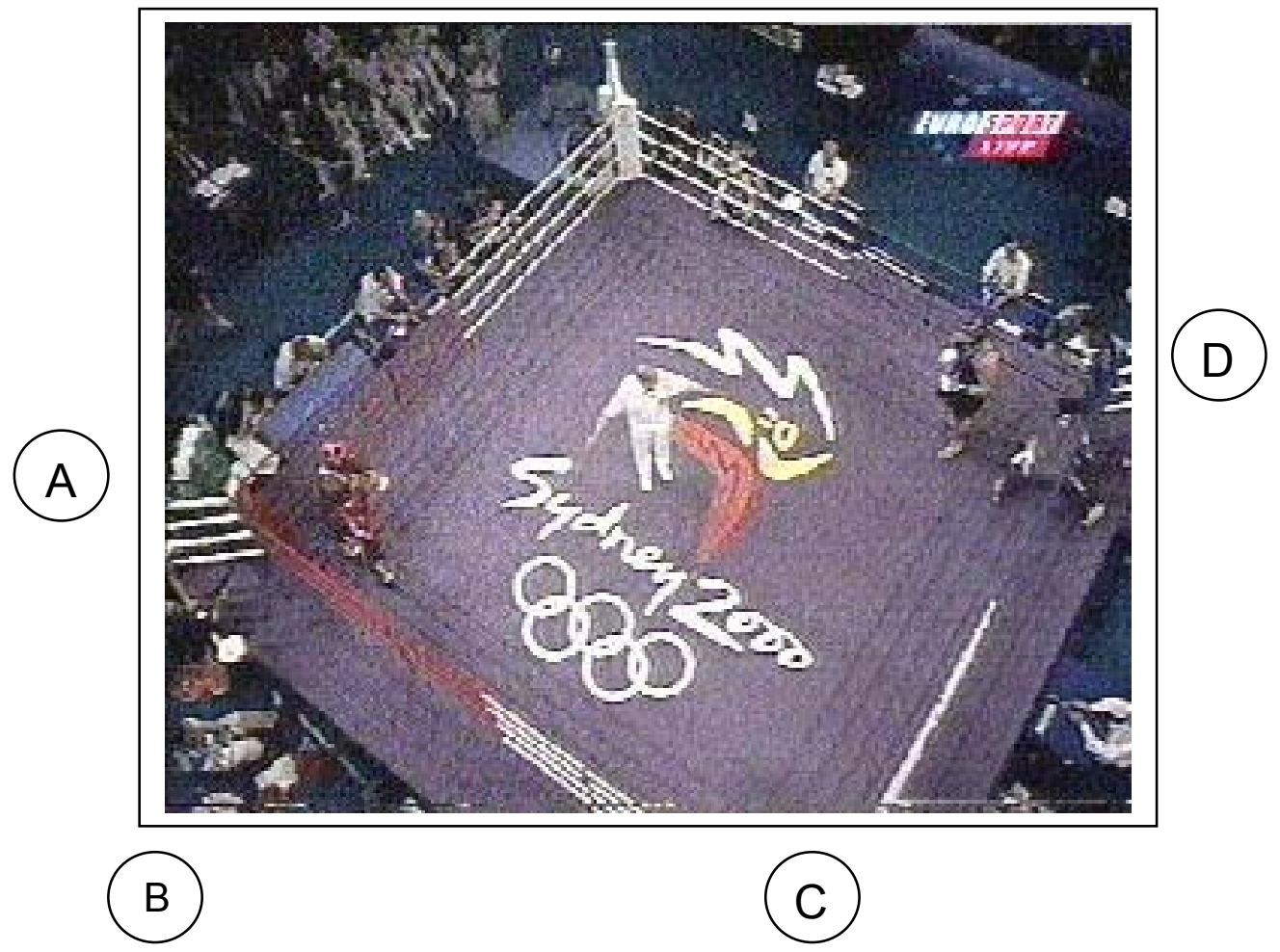

Abb. 38: Positionierung der einzelnen Kameras aus der Sicht der Deckenkamera (eigene Darstellung nach Eurosport) 


\subsection{Validität}

Die Validität besagt, dass das gemessen wurde, was man vorgibt zu messen. Da dieses eine Analyse zu Finten ist und Finten bestimmt wurden, ist die Arbeit logisch valid. Allerdings konnten aufgrund der Fernsehbilder nicht alle Finten analysiert werden. Die Finten mit den Augen konnten nicht in die Analyse einbezogen werden. Hierdurch fehlen drei von 22 möglichen Fintenarten, da 19 verschiedene Finten in die Analyse einbezogen wurden. (vgl. Abb. 36 und 37) Zwar sind diese Finten mit den Augen sehr wichtig, da sie parallel und additiv zu den anderen Finten einsetzbar sind, aber auf der anderen Seite sind es eben nur 17,6 \% der möglichen Fintenarten. Insgesamt kann die Analyse somit noch als valid gelten (vgl. KRÜGER, 1985); man sollte sich jedoch immer daran erinnern, dass eine wichtige Fintenart fehlt.

Die Ergebnisse zeigen zudem, dass die Reliabilität der Untersuchung hinreichend groß ist. Andere als die 19 dargestellten Finten wurden jedoch nicht erfasst. Formen der Bewegung des Kopfes und der Augen wurden nicht erfasst und waren durch das System nicht erfassbar. Aus der Befragung der Boxer oder deren Trainer ließen sich vielleicht noch weitere Finten erfassen und die Validität steigern. Auch berücksichtigt mein differenziertes, quantitatives Verfahren keine qualitativen Unterschiede und auch keine quantitativ in Bruchteilen von Sekunden messbaren Unterschiede.

Aber auch so hat die Untersuchung wichtige Aufschlüsse gegeben. Da durch die Videoanalyse bei einer Aufnahmegeschwindigkeit von 25 Bildern/Sek. kleinere Zeiteinheiten ohnehin von der Analyse nicht erfassbar sind, lassen sie sich wegen der komplizierten Situation von Bildrechten bei olympischen Turnieren nicht noch weiter verkürzen. Unter den gegebenen Umständen können daher die Ergebnisse mit den obigen Einschränkungen als valid gelten. 
Ich habe darauf die einfachen Finten zuerst mit den Beinen, dann mit dem Rumpf und zu Abschluss mit den Armen analysiert. Danach habe ich die kombinierten Finten mit den Beinen und dem Rumpf, dann mit den Beinen und den Armen (Halber Schritt nach vorne, Halber Schritt zurück) analysiert.

\subsection{Objektivität}

Für die Forschung ist es nun wichtig, die Objektivität der Analysen zu bestimmen. Für die Auswerterobjektivität muss (BALLREICH, 1970, 30f.) neben mir noch ein weiterer Experte das Analysesystem ,lernen" und auf die Kämpfe anwenden. Damit kann ich dann einen Objektivitätskoeffizienten berechnen. Deshalb habe ich 5 Kämpfe der Viertelfinale zu je vier Runden à zwei Minuten analysiert (1. Japan - Kuba im Bantamgewicht; 2. Korea - Turkei im Weltergewicht; 3. U.S.A. - Ägypten im Weltergewicht; 4. Uzbekistan - Deutschland im Halbmittelgewicht; 5. Dominikanische Republik - Österreich im Mittelgewicht. Siehe auch Anhang 6.)

Der Boxtrainer GEGHAM HAKOBIAN aus Armenien hat dieselben fünf Kämpfe unabhängig von mir analysiert. Innerhalb von 11 Jahren Boxerfahrung hat er fünfmal die Armenische Meisterschaft im Boxen gewonnen, in den Gewichtklassen $54 \mathrm{~kg}$ und $63 \mathrm{~kg}$. Er ist Sportmeister der Republik Armenien und ist seit 2001 Trainer des Sportclubs Arsenal in Armenien. Er war zum Zeitpunkt der Analyse zu Studienzwecken in Göttingen und hat dieselben Videogeräte benutzt. Ich habe inm in mein Finten-Analyse-System vier Stunden lang eingewiesen. Dann hat er unabhängig von Untersucher selbständig die Analyse des Videomaterials der fünf Kämpfe durchgeführt (25 Stunden). Die Gesamtzahl der Finten habe ich in die Rangfolgen 1 und 2 übertragen. Der Rangkorrelationskoeffizient beträgt $R=0,991$, d.h. es besteht eine sehr hohe Objektivität bezogen auf das analysierte Material. (vgl. KRÜGER, 1985) Im Weiteren sind jedoch nur meine Daten in die Berechnung eingegangen. Aufgrund der oben aufgelisteten Daten habe ich die Objektivitätskoeffizienten berechnet: 
Tab 1: Berechnung des Rangkorrelationskoeffizienten

\begin{tabular}{|c|c|c|c|c|c|c|}
\hline Finten & $\begin{array}{c}\text { Mittelwert die } \\
\text { Finten X1 }\end{array}$ & $\begin{array}{c}\text { Mittelwert die } \\
\text { Finten X2 }\end{array}$ & Ränge X1 & Ränge X2 & di & $\mathbf{d i}^{2}$ \\
\hline HV & 98 & 96 & 18 & 18 & 0 & 0 \\
\hline HR & 66 & 67 & 17 & 17 & 0 & 0 \\
\hline $\mathbf{A b}$ & 35 & 34 & 9 & 9 & 0 & 0 \\
\hline ValR & 42 & 44 & 12 & 13 & -1 & 1 \\
\hline VaR & 23 & 22 & 3 & 3 & 0 & 0 \\
\hline VarR & 62 & 63 & 16 & 16 & 0 & 0 \\
\hline KFi & 55 & 56 & 15 & 15 & 0 & 0 \\
\hline KFa & 16 & 15 & 2 & 2 & 0 & 0 \\
\hline KgSA & 48 & 47 & 14 & 14 & 0 & 0 \\
\hline BSvA & 32 & 33 & 8 & 8 & 0 & 0 \\
\hline ValBR & 28 & 26 & 5 & 4 & 1 & 1 \\
\hline VaBR & 36 & 37 & 10 & 11 & -1 & 1 \\
\hline VarBR & 30 & 30 & 7 & 7 & 0 & 0 \\
\hline KgSBAV & 37 & 36 & 11 & 10 & 1 & 1 \\
\hline SSV & 43 & 43 & 13 & 12 & -1 & 1 \\
\hline BSvBAV & 0 & 0 & 0 & 0 & 0 & 0 \\
\hline KgSBAZ & 27 & 28 & 4 & 6 & -2 & 4 \\
\hline SSZ & 29 & 27 & 6 & 5 & 1 & 1 \\
\hline BSvBAZ & 8 & 8 & 1 & 1 & 0 & 0 \\
\hline Summe & & & & & & 10 \\
\hline
\end{tabular}

Der R-Wert = 0,399 signifikant auf dem Niveau von 0,05 (Zweiseitig)

Der R-Wert $=$ 0,564 signifikant auf dem Niveau von 0,01 (Zweiseitig)

$R=0,991>0,564$, d.h. das Ergebnis ist hoch signifikant auf dem 0,01 Niveau. 


\section{Statistische Auswertung}

Die statistische Auswertung der Daten erfolgte im Einzelnen manuell. Es handelt sich um folgende Maße und Verfahren:

\section{1: Deskriptive Statistik}

\section{Arithmetisches Mittel, Mittelwert}

Der Mittelwert ist das arithmetische Mittel der Messwerte. Er berechnet sich aus der Summe der Messwerte, geteilt durch ihre Anzahl.

\section{2: Analytische Statistik}

Die analytische Statistik ist neben der deskriptiven der zweite Teilaspekt der Statistik. Sie befasst sich mit der Überprüfung von Hypothesen und ermöglicht objektive Entscheidungen über deren Brauchbarkeit.

\section{Irrtumswahrscheinlichkeit (p)}

Sie gibt an, mit welcher Wahrscheinlichkeit man sich irren würde, wenn man die fragliche Hypothese akzeptiert. Um die Hypothese annehmen zu können, sollte p möglichst klein sein. Die Grenze, die von p nicht überschritten werden darf, wird als „Signifikanzniveau“ bezeichnet und wird üblicherweise auf $5 \%$ festgelegt. Daraus ergibt sich: $p>0,05$ - nicht signifikant, $p \leq 0,05$ - signifikant, $P$ $\leq 0,01$ - hoch signifikanț.

HO (Die Verteilung gleicht einer Normalverteilung) und die Alternativhypothese

H1 (Die Verteilung gleicht nicht einer Normalverteilung) werden formuliert.

Bei einer Signifikanz $\mathbf{p}<0,05$ muss die Nullhypothese abgelehnt und die Alternativhypothese angenommen werden. Bei Werten der Signifikanz von $p>$ 0,05 muss die Nullhypothese angenommen und die Alternativhypothese abgelehnt werden. (vgl. SIEGEL, CASTELLAN, 1988; BORTZ, 1999; ROHLAND, 2000) 


\section{WILCOXON- Test}

Das ist ein nicht parametrischer Test für gepaarte Stichproben. Es wird neben der Richtung der Unterschiede innerhalb der Datenpaare auch die Größe der Unterschiede in Betracht gezogen, wobei ein Paar mit größerer Differenz stärker gewichtet wird als ein Paar mit geringerem Unterschied. Daraus lässt sich eine Rangordnung ableiten. Weiterhin wird die Irrtumswahrscheinlichkeit $p$ berechnet. (vgl. BÖS, 1986; HARMS, 1998; ROHLAND, 2000)

\section{Chi- Quadrat- Test ( $x^{2}-$ Test)}

- Der Chi- Quadrat- Test leistet:

Gegebene Daten mit relativen Häufigkeiten, sind die Daten korreliert mit einer (gegebenen) Wahrscheinlichkeit?

- Die Wahrscheinlichkeit, dass Variable unabhängig sind, heißt SignifikanzNiveau.

- Beispiel: Wenn wir das Signifikanz-Niveau von 95\% auf 99\% verändern, heißt das:

Wir wollen noch sicherer sein, dass die zugrundeliegenden Daten realistisch sind.

- Diverse Annahmen müssen erfüllt sein, die hier nicht besprochen werden.

- Normalisierte Abweichung vom Erwartungswert

- $\mathbf{x}^{2}=$ '0' bedeutet 'Alle Variablen sind unabhängig',

- $\mathbf{x}^{2}>$ 'Schwellenwert' bedeutet nicht unabhängig mit bestimmtem SignifikanzNiveau. (vgl. RUDI; GÜNTER u. a., 1976; STEMMLER; BECHER, 1980; JOHN, 1997; GÖTZ, 2001) 


\section{Ergebnisse und Schlussfolgerung}

\subsection{Der Sieger verwendet mehr Finten}

Tabelle 2 zeigt den Unterschied zwischen den Siegern und den Verlierern bei der Verwendung der neunzehn Finten sowie die Signifikanzen aus dem Wilcoxon-Test.

Tab 2: Anzahl der von Siegern $(n=28)$ bzw. Verlierern $(n=28)$ verwendeten Finten.

\begin{tabular}{|c|c|c|c|c|c|c|}
\hline Finten & Sieger & Verlierer & di & Rangplatz & $d i<0$ & $d i>0$ \\
\hline HV & 293 & 202 & 91 & 10 & & 10 \\
\hline HR & 222 & 122 & 100 & 12 & & 12 \\
\hline$A b$ & 157 & 102 & 55 & 9 & & 9 \\
\hline ValR & 251 & 142 & 109 & 13 & & 13 \\
\hline VaR & 4 & 1 & 3 & 1 & & 1 \\
\hline VarR & 211 & 115 & 96 & 11 & & 11 \\
\hline $\mathrm{KFi}$ & 139 & 102 & 37 & 7 & & 7 \\
\hline KFa & 31 & 21 & 10 & 4 & & 4 \\
\hline KgSA & 383 & 219 & 164 & 14 & & 14 \\
\hline BSvA & 57 & 38 & 19 & 6 & & 6 \\
\hline ValBR & 320 & 123 & 197 & 17 & & 17 \\
\hline VaBR & 0 & 0 & 0 & 0 & & 0 \\
\hline VarBR & 274 & 109 & 165 & 15 & & 15 \\
\hline KgSBAV & 508 & 242 & 266 & 18 & & 18 \\
\hline SSV & 350 & 180 & 170 & 16 & & 16 \\
\hline BSvBAV & 12 & 4 & 8 & 3 & & 3 \\
\hline KgSBAZ & 73 & 33 & 40 & 8 & & 8 \\
\hline SSZ & 21 & 7 & 14 & 5 & & 5 \\
\hline BSvBAZ & 5 & 0 & 5 & 2 & & 2 \\
\hline Summe & & & & 171 & $\mathrm{~T}=0$ & $T^{\prime}=171$ \\
\hline
\end{tabular}

Der T- Wert $=40$ signifikant auf dem Niveau von 0,05 (Zweiseitig)

Der T- Wert = 28 signifikant auf dem Niveau von 0,01 (Zweiseitig)

$\mathrm{T}=0<28$, daher hoch signifikant auf dem 0,01 Niveau.

In der Tab. 2 sind die Finten für die Sieger und die Finten für die Verlierer aufgezeigt. Die Differenzen zwischen den Finten für Sieger und Verlierer und die Rangplätze von di und di $<0$ oder di $>0$ sind ebenfalls dargestellt. 


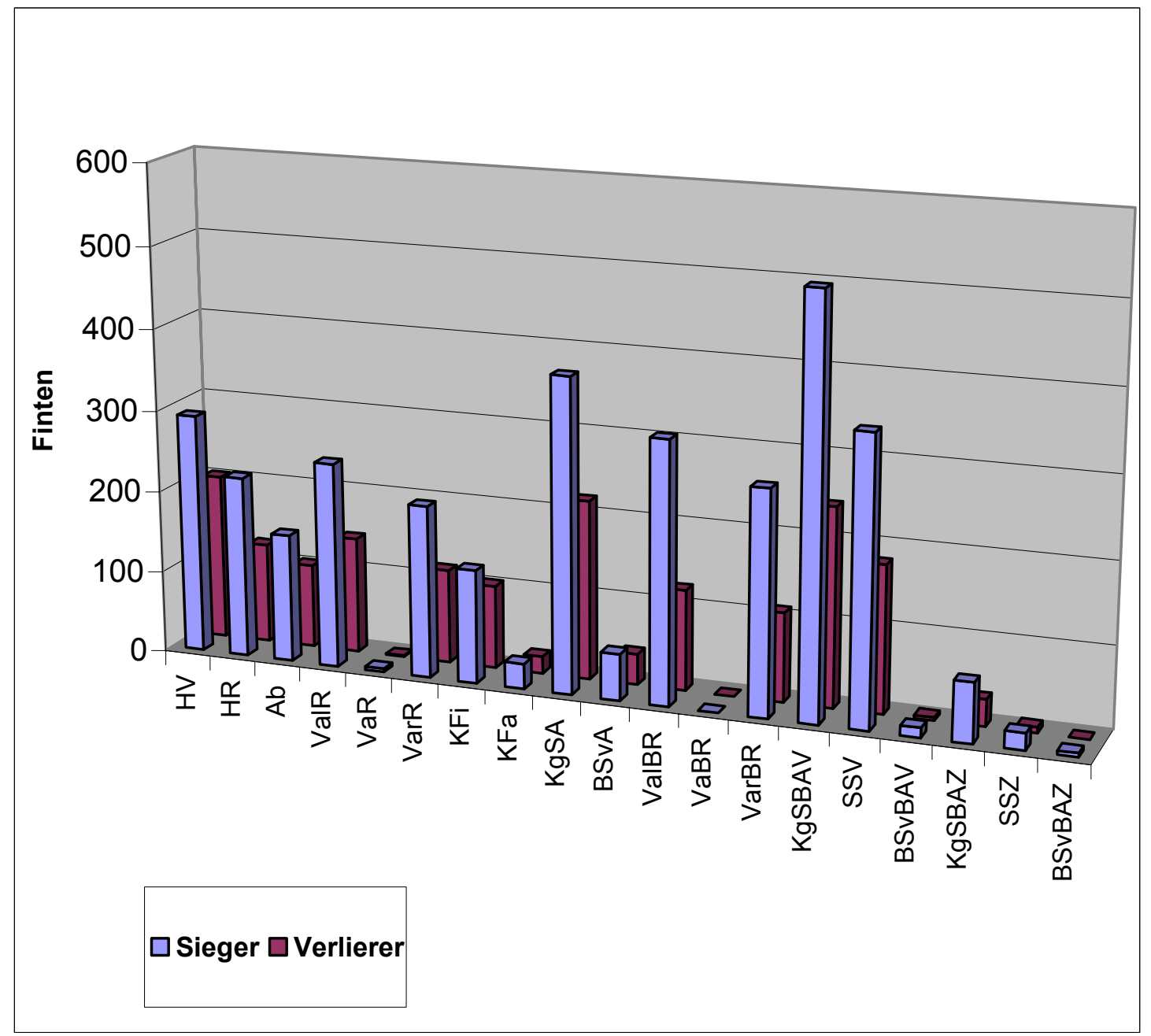

Abb. 39: Der Sieger $(n=28)$ verwendet mehr Finten als der Verlierer $(n=28)$.

Abb. 39 zeigt den Unterschied zwischen den Siegern und den Verlierern bei der Verwendung der neunzehn Finten. 


\subsection{Der Sieger verwendet mehr Kombinierte Finten}

Tabelle 3 zeigt den Unterschied zwischen den Siegern und den Verlierern bei der Verwendung der neun Kombinierten Finten sowie die Signifikanzen aus dem Wilcoxon-Test.

Tab 3: Anzahl der von Siegern $(n=28)$ bzw. Verlierern $(n=28)$ verwendeten Kombinierten Finten.

\begin{tabular}{|c|c|c|c|c|c|c|}
\hline $\begin{array}{l}\text { Kombinierte } \\
\text { Finten }\end{array}$ & Sieger & Verlierer & di & Rangplatz & $d i<0$ & $d i>0$ \\
\hline ValBR & 320 & 123 & 197 & 7 & & 7 \\
\hline VaBR & 0 & 0 & 0 & 0 & & \\
\hline VarBR & 274 & 109 & 165 & 5 & & 5 \\
\hline KgSBAV & 508 & 242 & 266 & 8 & & 8 \\
\hline SSV & 350 & 180 & 170 & 6 & & 6 \\
\hline BSvBAV & 12 & 4 & 8 & 2 & & 2 \\
\hline KgSBAZ & 73 & 33 & 40 & 4 & & 4 \\
\hline SSZ & 21 & 7 & 14 & 3 & & 3 \\
\hline BSvBAZ & 5 & 0 & 5 & 1 & & 1 \\
\hline Summe & & & & 36 & $T=0$ & $T^{\prime}=36$ \\
\hline
\end{tabular}

Der T- Wert $=4$ signifikant auf dem Niveau von 0,05 (Zweiseitig)

Der T- Wert $=0$ signifikant auf dem Niveau von 0,01 (Zweiseitig) $\mathrm{T}=0$, d.h. das Ergebnis ist hoch signifikant auf dem 0,01 Niveau.

In der Tab. 3 sind die Kombinierten Finten für die Sieger und die Kombinierten Finten für die Verlierer aufgezeigt. Die Differenzen zwischen den Kombinierten Finten für Sieger und Verlierer und die Rangplätze von di und di $<0$ oder di $>0$ sind ebenfalls dargestellt. 


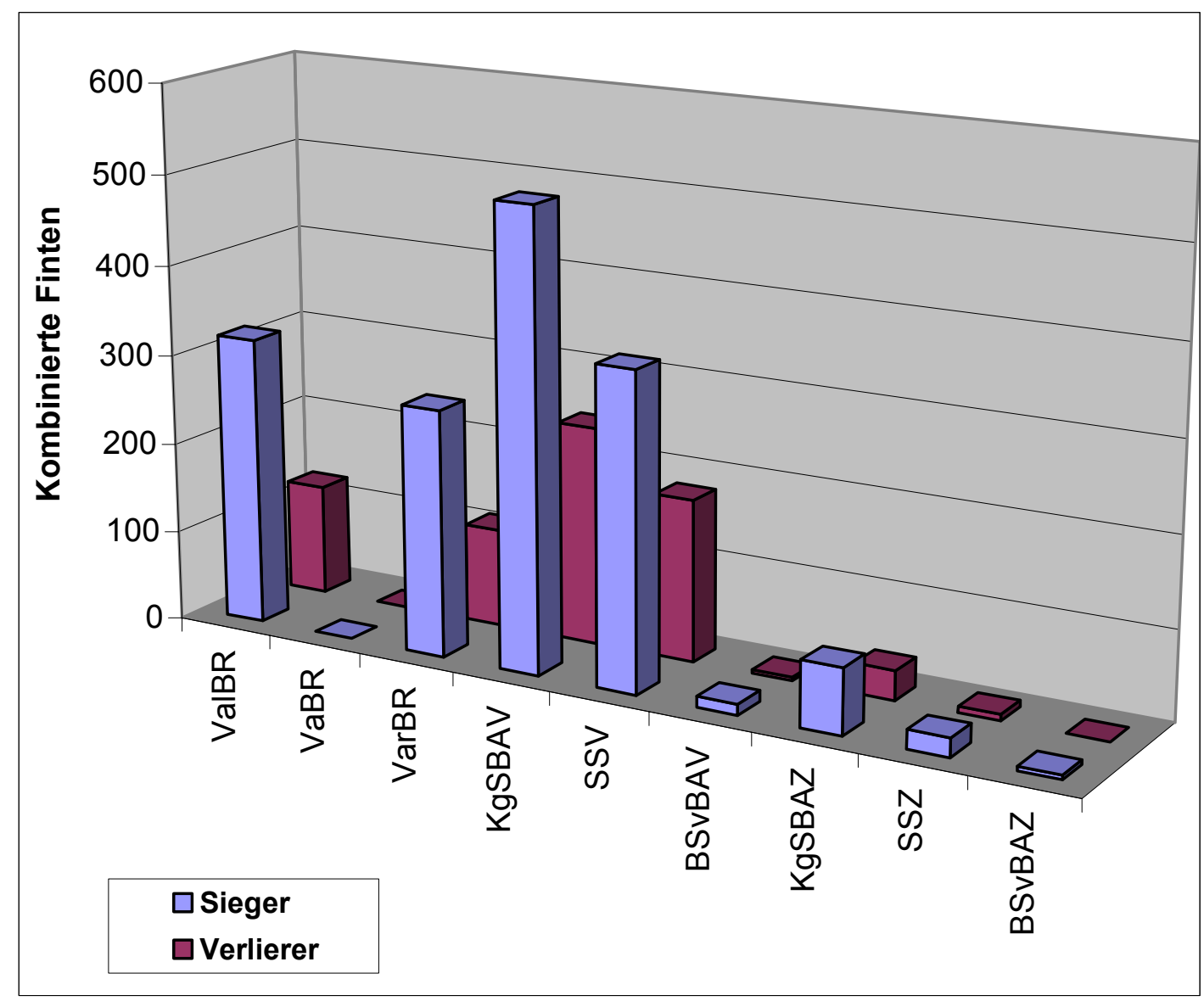

Abb. 40: Der Sieger $(n=28)$ verwendet mehr Kombinierte Finten als der Verlierer $(n=28)$.

Abb. 40 zeigt den Unterschied zwischen den Siegern und den Verlierern bei der Verwendung der neun Kombinierten Finten. 


\subsection{Der Sieger verwendet mehr variable Kombinierte Finten}

Tabelle 4 zeigt den Unterschied zwischen den Siegern und den Verlierern bei der Verwendung der neun variablen Kombinierten Finten sowie die Signifikanzen aus dem Wilcoxon-Test.

Tab 4: Der Sieger $(n=28)$ verwendet mehr variable Kombinierte Finten als der Verlierer $(\mathbf{n}=\mathbf{2 8})$.

\begin{tabular}{|c|c|c|c|c|c|c|}
\hline $\begin{array}{l}\text { Kombinierte } \\
\text { Finten }\end{array}$ & Sieger & Verlierer & di & $\begin{array}{l}\text { Rang- } \\
\text { platz }\end{array}$ & $d i<0$ & $d i>0$ \\
\hline ValBR & 11 & 12 & -1 & 4 & 4 & \\
\hline VaBR & 0 & 0 & 0 & 0 & & \\
\hline VarBR & 12 & 11 & 1 & 4 & & 4 \\
\hline KgSBAV & 12 & 11 & 1 & 4 & & 4 \\
\hline SSV & 12 & 11 & 1 & 4 & & 4 \\
\hline BSvBAV & 2 & 1 & 1 & 4 & & 4 \\
\hline KgSBAZ & 8 & 6 & 2 & 8 & & 8 \\
\hline SSZ & 4 & 3 & 1 & 4 & & 4 \\
\hline BSvBAZ & 1 & 0 & 1 & 4 & & 4 \\
\hline Summe & & & & 36 & $\mathrm{~T}=4$ & $T^{\prime}=32$ \\
\hline
\end{tabular}

Der T- Wert $=4$ signifikant auf dem Niveau von 0,05 (Zweiseitig)

Der T- Wert $=0$ signifikant auf dem Niveau von 0,01 (Zweiseitig) $\mathrm{T}=4$, d.h. das Ergebnis ist signifikant auf dem 0,05 Niveau.

In der Tab. 4 sind die variablen Kombinierten Finten für die Sieger und die variablen Kombinierten Finten für die Verlierer aufgezeigt. Die Differenzen zwischen die variablen Kombinierten Finten für Sieger und Verlierer und die Rangplätze von di und di $<0$ oder di $>0$ sind ebenfalls dargestellt. 


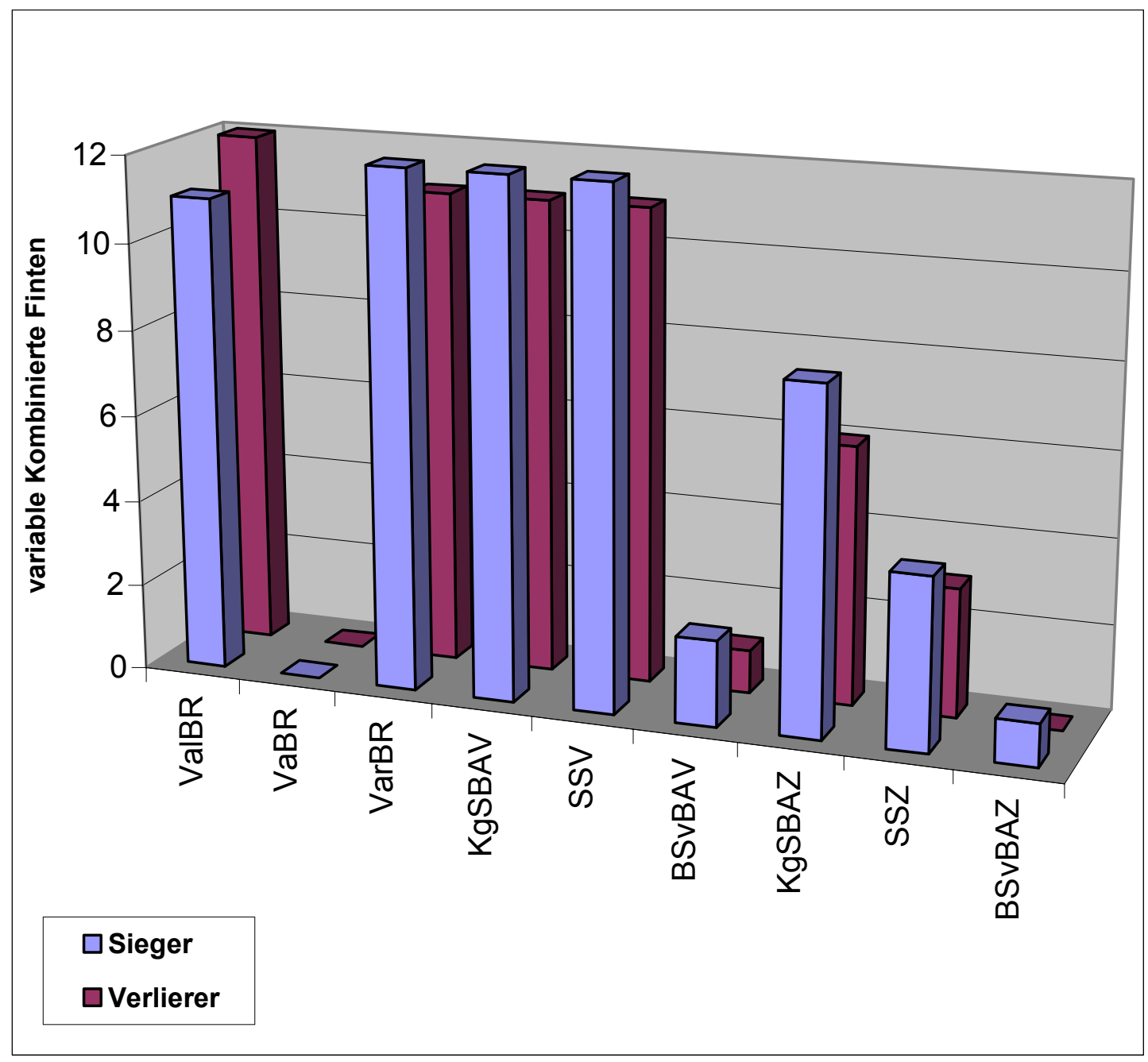

Abb. 41: Der Sieger $(n=28)$ verwendet mehr variable Kombinierte Finten als der Verlierer $(n=28)$.

Abb. 41 zeigt den Unterschied zwischen den Siegern und den Verlierern bei der Verwendung der neun variablen Kombinierten Finten. 


\subsection{Schwere Boxer verwenden Finten häufiger als leichte Bo- xer}

Boxer der sechs schwersten Gewichtsklassen (Weltergewicht, Halbmittelgewicht, Mittelgewicht, Halbschwergewicht, Schwergewicht und Superschwergewicht) verwenden Finten häufiger als Boxer der sechs leichtesten Gewichtsklassen (Halbfliegengewicht, Fliegengewicht, Bantamgewicht, Federgewicht, Leichtgewicht und Halbweltergewicht).

Tab 5: Boxer der sechs schwersten Gewichtsklassen $(n=28)$ verwenden Finten häufiger als Boxer der sechs leichtesten Gewichtsklassen $(n=28)$.

\begin{tabular}{|c|c|c|c|c|c|c|}
\hline Finten & $\begin{array}{l}\text { leichte } \\
\text { Boxer }\end{array}$ & $\begin{array}{c}\text { schwere } \\
\text { Boxer }\end{array}$ & di & Rangplatz & $d i<0$ & $d i>0$ \\
\hline HV & 238 & 257 & -19 & 9 & 9 & \\
\hline HR & 171 & 173 & -2 & 2 & 2 & \\
\hline$A b$ & 113 & 146 & -33 & 12 & 12 & \\
\hline ValR & 155 & 248 & -93 & 15 & 15 & \\
\hline VaR & 0 & 5 & -5 & 4 & 4 & \\
\hline VarR & 126 & 200 & -74 & 14 & 14 & \\
\hline KFi & 48 & 193 & -145 & 18 & 18 & \\
\hline KFa & 32 & 31 & -1 & 1 & 1 & \\
\hline KgSA & 295 & 307 & -12 & 7 & 7 & \\
\hline BSvA & 45 & 50 & -5 & 4 & 4 & \\
\hline ValBR & 173 & 270 & -97 & 16 & 16 & \\
\hline VaBR & 0 & 0 & 0 & 0 & & \\
\hline VarBR & 134 & 249 & -115 & 17 & 17 & \\
\hline KgSBAV & 340 & 410 & -70 & 13 & 13 & \\
\hline SSV & 250 & 280 & -30 & 11 & 11 & \\
\hline BSvBAV & 0 & 16 & -16 & 8 & 8 & \\
\hline KgSBAZ & 57 & 49 & 8 & 6 & & 6 \\
\hline SSZ & 25 & 3 & 22 & 10 & & 10 \\
\hline BSvBAZ & 0 & 5 & -5 & 4 & 4 & \\
\hline Summe & & & & 171 & $T=155$ & $T^{\prime}=16$ \\
\hline
\end{tabular}

Der T- Wert $=40$ signifikant auf dem Niveau von 0,05 (Zweiseitig)

Der T- Wert $=28$ signifikant auf dem Niveau von 0,01 (Zweiseitig)

$\mathrm{T}=16<28$, d.h. das Ergebnis ist hoch signifikant auf dem 0,01 Niveau.

In der Tab. 5 sind die Finten für die leichten Boxer und die Finten für die schweren Boxer aufgezeigt. Die Differenzen zwischen den Finten für die leichten Boxer und den schweren Boxern und die Rangplätze von di und di $<0$ oder di $>0$ sind ebenfalls dargestellt. 


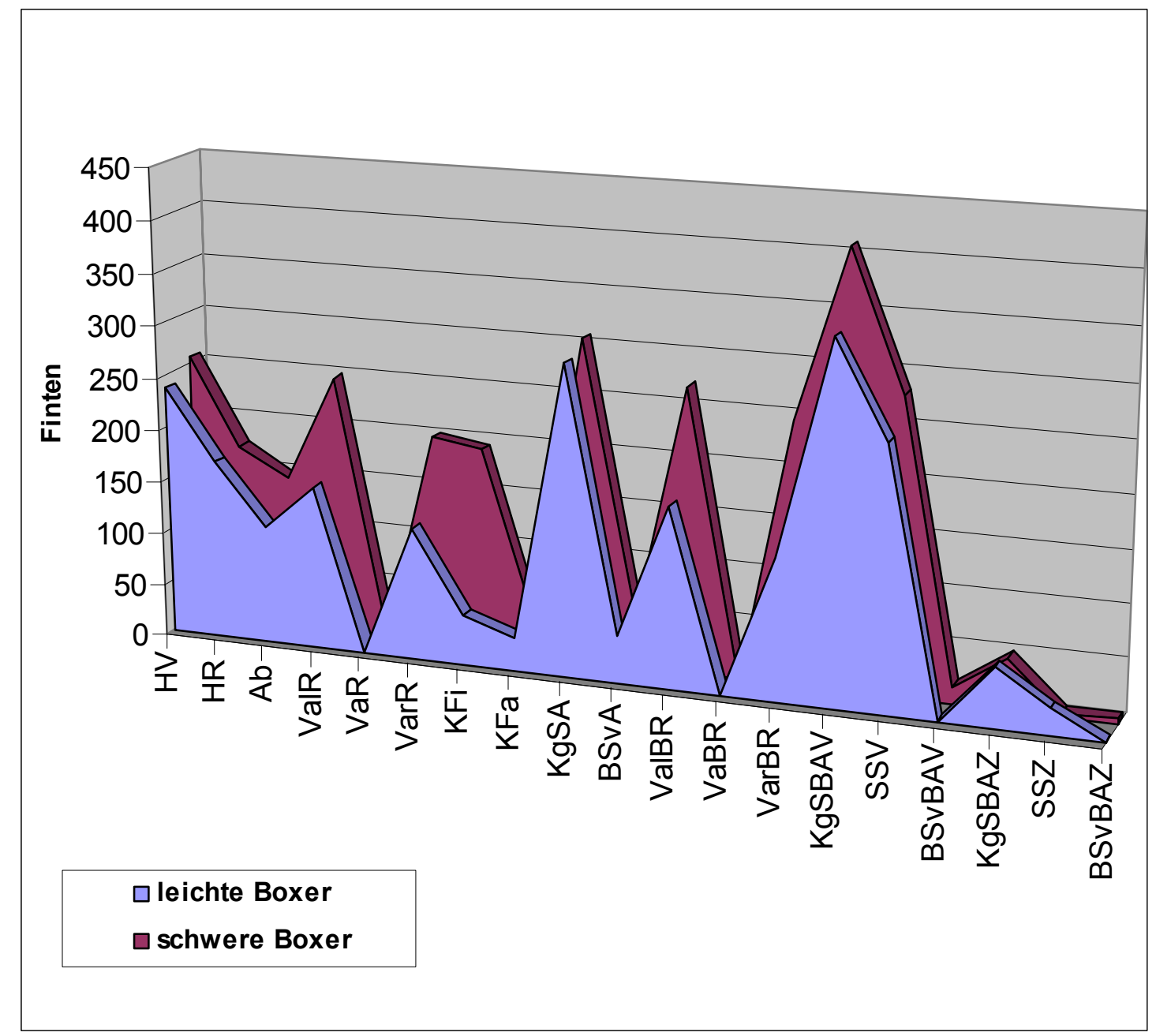

Abb. 42: Boxer der sechs schwersten Gewichtsklassen (Weltergewicht, Halbmittelgewicht, Mittelgewicht, Halbschwergewicht, Schwergewicht und Superschwergewicht) $(n=28)$ verwenden Finten häufiger als Boxer der sechs leichtesten Gewichtsklassen (Halbfliegengewicht, Fliegengewicht, Bantamgewicht, Federgewicht, Leichtgewicht und Halbweltergewicht) $(n=28)$.

Abb. 42 zeigt den Unterschied zwischen den schweren Boxern und den leichten Boxern bei der Verwendung der neunzehn Finten. 
Boxer der fünf schwersten Gewichtsklassen (Halbmittelgewicht, Mittelgewicht, Halbschwergewicht, Schwergewicht und Superschwergewicht) verwenden Finten häufiger als Boxer der fünf leichtesten Gewichtsklassen (Halbfliegengewicht, Fliegengewicht, Bantamgewicht, Federgewicht und Leichtgewicht). Die beiden mittleren Gewichtsklassen sind weggelassen, um die Unterschiede zwischen "leicht" und "schwer" zu verdeutlichen.

Tab 6: Boxer der fünf schwersten Gewichtsklassen ( $n=26)$ verwenden Finten häufiger als Boxer der fünf leichtesten Gewichtsklassen ( $n=26)$.

\begin{tabular}{|c|c|c|c|c|c|c|}
\hline Finten & $\begin{array}{l}\text { leichte } \\
\text { Boxer }\end{array}$ & $\begin{array}{l}\text { schwere } \\
\text { Boxer }\end{array}$ & di & Rangplatz & $d i<0$ & $\mathrm{di}>0$ \\
\hline HV & 205 & 236 & -31 & 9 & 9 & \\
\hline HR & 158 & 165 & -7 & 4 & 4 & \\
\hline$A b$ & 99 & 137 & -38 & 12 & 12 & \\
\hline ValR & 149 & 225 & -76 & 15 & 15 & \\
\hline VaR & 0 & 5 & -5 & 1,5 & 1,5 & \\
\hline VarR & 125 & 178 & -53 & 13 & 13 & \\
\hline KFi & 48 & 193 & -145 & 18 & 18 & \\
\hline $\mathrm{KFa}$ & 22 & 31 & -9 & 5 & 5 & \\
\hline KgSA & 258 & 292 & -34 & 10 & 10 & \\
\hline BSvA & 43 & 49 & -6 & 3 & 3 & \\
\hline ValBR & 154 & 251 & -97 & 16 & 16 & \\
\hline VaBR & 0 & 0 & 0 & 0 & 0 & \\
\hline VarBR & 118 & 231 & -113 & 17 & 17 & \\
\hline KgSBAV & 314 & 382 & -68 & 14 & 14 & \\
\hline SSV & 228 & 263 & -35 & 11 & 11 & \\
\hline BSvBAV & 0 & 13 & -13 & 7 & 7 & \\
\hline KgSBAZ & 39 & 49 & -10 & 6 & 6 & \\
\hline SSZ & 19 & 3 & 16 & 8 & & 8 \\
\hline BSvBAZ & 0 & 5 & -5 & 1,5 & 1,5 & \\
\hline Summe & & & & 171 & $T=163$ & $\mathrm{~T}=8$ \\
\hline
\end{tabular}

Der T- Wert $=40$ signifikant auf dem Niveau von 0,05 (Zweiseitig)

Der T- Wert = 28 signifikant auf dem Niveau von 0,01 (Zweiseitig)

$\mathrm{T}=8<28$, d.h. das Ergebnis ist hoch signifikant auf dem 0,01 Niveau.

In der Tab. 6 sind die Finten für die leichten Boxer und die Finten für die schweren Boxer aufgezeigt. Die Differenzen zwischen den Finten für die leichten Boxer und die schweren Boxer und die Rangplätze von di und di $<0$ oder di $>0$ sind ebenfalls dargestellt. 


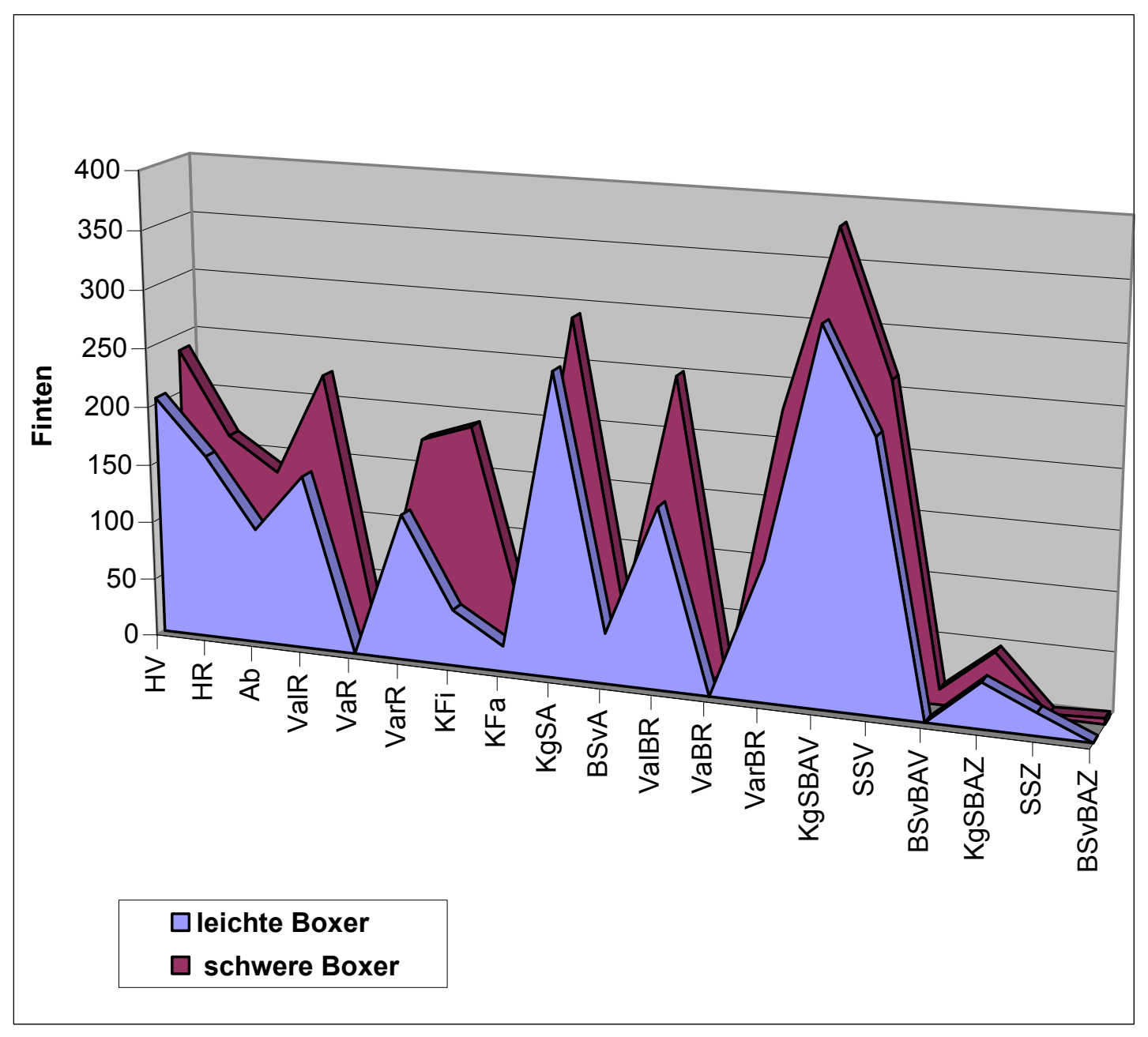

Abb. 43: Boxer der fünf schwersten Gewichtsklassen (Halbmittelgewicht, Mittelgewicht, Halbschwergewicht, Schwergewicht und Superschwergewicht) $(n=26)$ verwenden Finten häufiger als Boxer der fünf leichtesten Gewichtsklassen (Halbfliegengewicht, Fliegengewicht, Bantamgewicht, Federgewicht und Leichtgewicht) $(n=26)$.

Abb. 43 zeigt den Unterschied zwischen den schweren Boxern und den leichten Boxern bei der Verwendung der neunzehn Finten. 
Boxer der vier schwersten Gewichtsklassen (Mittelgewicht, Halbschwergewicht, Schwergewicht und Superschwergewicht) verwenden Finten häufiger als Boxer der vier leichtesten Gewichtsklassen (Halbfliegengewicht, Fliegengewicht, Bantamgewicht und Federgewicht).

Tab 7: Boxer der vier schwersten Gewichtsklassen $(n=22)$ verwenden Finten häufiger als Boxer der vier leichtesten Gewichtsklassen $(n=22)$.

\begin{tabular}{|c|c|c|c|c|c|c|}
\hline Finten & $\begin{array}{l}\text { leichte } \\
\text { Boxer }\end{array}$ & $\begin{array}{l}\text { schwere } \\
\text { Boxer }\end{array}$ & di & Rangplatz & $d i<0$ & $\mathrm{di}>0$ \\
\hline HV & 167 & 189 & -22 & 10 & 10 & \\
\hline HR & 118 & 131 & -13 & 7 & 7 & \\
\hline$A b$ & 71 & 102 & -31 & 11 & 11 & \\
\hline VaIR & 119 & 164 & -45 & 15 & 15 & \\
\hline VaR & 0 & 5 & -5 & 1,5 & 1,5 & \\
\hline VarR & 98 & 137 & -39 & 14 & 14 & \\
\hline $\mathrm{KFi}$ & 41 & 176 & -135 & 18 & 18 & \\
\hline $\mathrm{KFa}$ & 19 & 29 & -10 & 4 & 4 & \\
\hline KgSA & 217 & 252 & -35 & 13 & 13 & \\
\hline BSvA & 41 & 47 & -6 & 3 & 3 & \\
\hline ValBR & 133 & 209 & -76 & 16 & 16 & \\
\hline VaBR & 0 & 0 & 0 & 0 & & \\
\hline VarBR & 103 & 192 & -89 & 17 & 17 & \\
\hline KgSBAV & 278 & 312 & -34 & 12 & 12 & \\
\hline SSV & 192 & 211 & -19 & 9 & 9 & \\
\hline BSvBAV & 0 & 12 & -12 & 6 & 6 & \\
\hline KgSBAZ & 37 & 48 & -11 & 5 & 5 & \\
\hline SSZ & 17 & 3 & 14 & 8 & & 8 \\
\hline BSvBAZ & 0 & 5 & -5 & 1,5 & 1,5 & \\
\hline Summe & & & & 171 & $\mathrm{~T}=163$ & $T^{\prime}=8$ \\
\hline
\end{tabular}

Der T- Wert $=40$ signifikant auf dem Niveau von 0,05 (Zweiseitig)

Der T- Wert $=28$ signifikant auf dem Niveau von 0,01 (Zweiseitig)

$\mathrm{T}=8<28$, d.h. das Ergebnis ist hoch signifikant auf dem 0,01 Niveau.

In der Tab. 7 sind die Finten für die leichten Boxer und die Finten für die schweren Boxer aufgezeigt. Die Differenzen zwischen den Finten für die leichten Boxer und die schweren Boxer und die Rangplätze von di und di $<0$ oder di $>0$ sind ebenfalls dargestellt. 


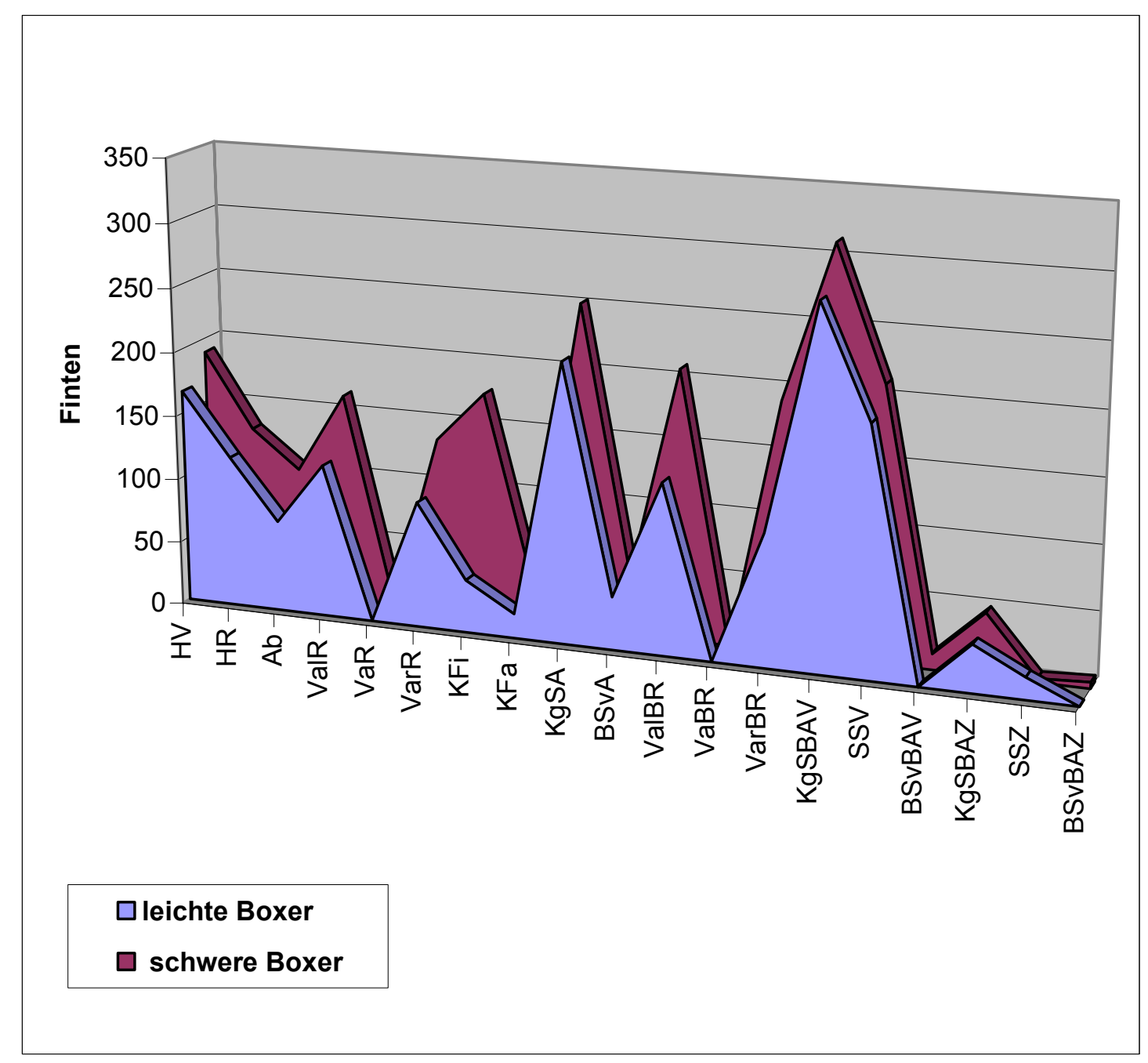

Abb. 44: Boxer der vier schwersten Gewichtsklassen (Mittelgewicht, Halbschwergewicht, Schwergewicht und Superschwergewicht) ( $n=22)$ verwenden Finten häufiger als Boxer der vier leichtesten Gewichtsklassen (Halbfliegengewicht, Fliegengewicht, Bantamgewicht und Federgewicht) $(n=22)$.

Abb. 44 zeigt den Unterschied zwischen den schweren Boxern und den leichten Boxern bei der Verwendung der neunzehn Finten. 
Boxer der drei schwersten Gewichtsklassen (Halbschwergewicht, Schwergewicht und Superschwergewicht) verwenden Finten häufiger als Boxer der drei leichtesten Gewichtsklassen (Halbfliegengewicht, Fliegengewicht und Bantamgewicht).

Tab 8: Boxer der drei schwersten Gewichtsklassen ( $n=18)$ verwenden Finten häufiger als Boxer der drei leichtesten Gewichtsklassen ( $n=18)$.

\begin{tabular}{|c|c|c|c|c|c|c|}
\hline Finten & $\begin{array}{l}\text { leichte } \\
\text { Boxer }\end{array}$ & $\begin{array}{c}\text { schwere } \\
\text { Boxer }\end{array}$ & di & Rangplatz & $d i<0$ & $d i>0$ \\
\hline HV & 131 & 147 & -16 & 7 & 7 & \\
\hline HR & 92 & 109 & -17 & 8,5 & 8,5 & \\
\hline$A b$ & 43 & 82 & -39 & 15 & 15 & \\
\hline ValR & 94 & 123 & -29 & 10 & 10 & \\
\hline VaR & 0 & 0 & 0 & 0 & 0 & \\
\hline VarR & 79 & 111 & -32 & 11 & 11 & \\
\hline $\mathrm{KFi}$ & 32 & 127 & -95 & 17 & 17 & \\
\hline KFa & 9 & 23 & -14 & 6 & 6 & \\
\hline KgSA & 164 & 199 & -35 & 12,5 & 12,5 & \\
\hline BSvA & 37 & 31 & 6 & 2 & & 2 \\
\hline ValBR & 118 & 154 & -36 & 14 & 14 & \\
\hline VaBR & 0 & 0 & 0 & 0 & & \\
\hline VarBR & 89 & 124 & -35 & 12,5 & 12,5 & \\
\hline KgSBAV & 198 & 253 & -55 & 16 & 16 & \\
\hline sSV & 142 & 125 & 17 & 8,5 & & 8,5 \\
\hline BSvBAV & 0 & 8 & -8 & 3 & 3 & \\
\hline KgSBAZ & 32 & 43 & -11 & 5 & 5 & \\
\hline SSZ & 12 & 3 & 9 & 4 & & 4 \\
\hline BSvBAZ & 0 & 5 & -5 & 1 & 1 & \\
\hline Summe & & & & 153 & $T=138,5$ & $T^{\prime}=14,5$ \\
\hline
\end{tabular}

Der T- Wert $=35$ signifikant auf dem Niveau von 0,05 (Zweiseitig)

Der T- Wert = 23 signifikant auf dem Niveau von 0,01 (Zweiseitig)

$\mathrm{T}=14,5<23$, d.h. das Ergebnis ist hoch signifikant auf dem 0,01 Niveau.

In der Tab. 8 sind die Finten für die leichten Boxer und die Finten für die schweren Boxer aufgezeigt. Die Differenzen zwischen den Finten für die leichten Boxer und die schweren Boxer und die Rangplätze von di und di $<0$ oder di $>0$ sind ebenfalls dargestellt. 


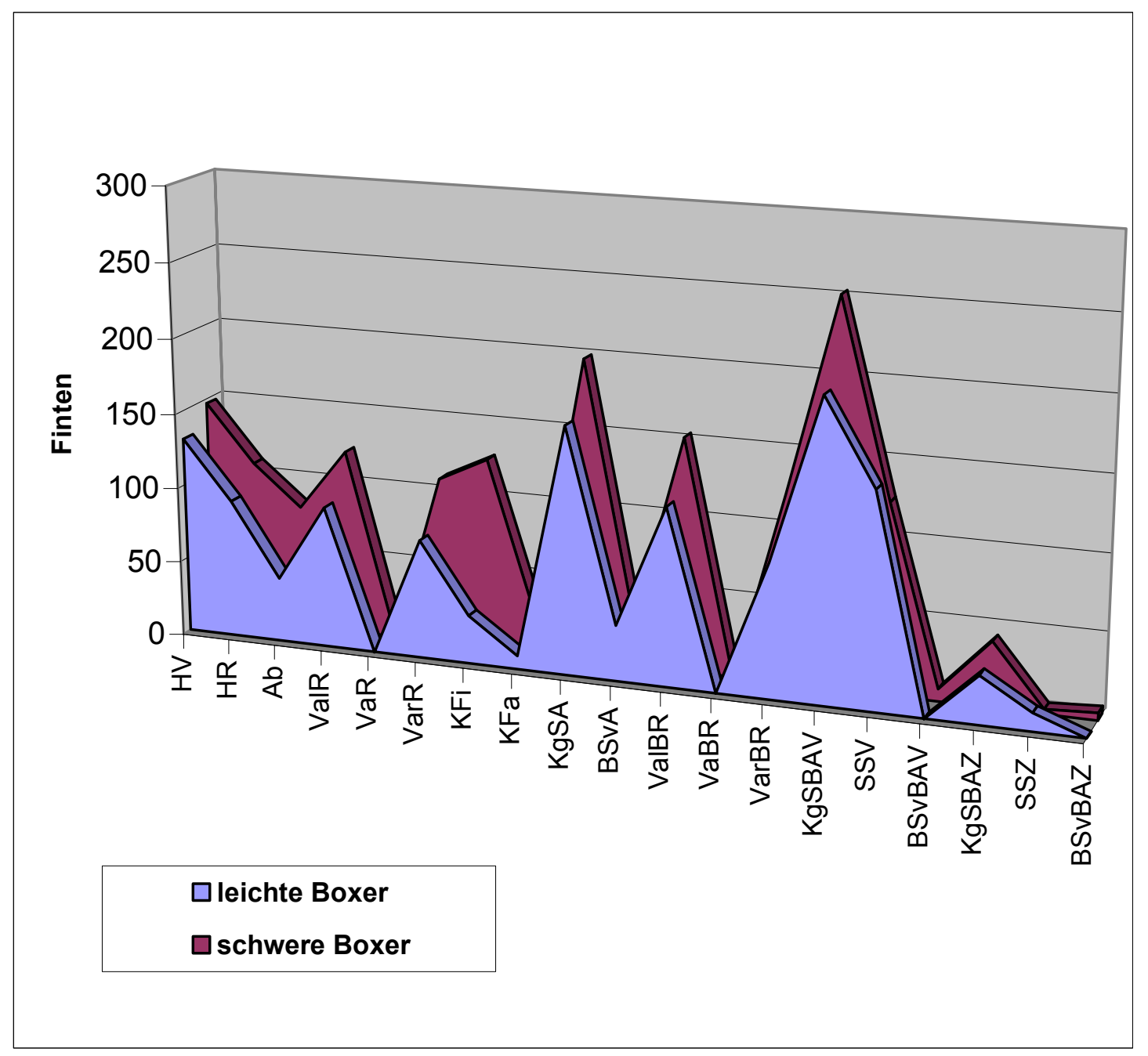

Abb. 45: Boxer der drei schwersten Gewichtsklassen (Halbschwergewicht, Schwergewicht und Superschwergewicht) $(n=18)$ verwenden Finten häufiger als Boxer der drei leichtesten Gewichtsklassen (Halbfliegengewicht, Fliegengewicht und Bantamgewicht) ( $n=18)$.

Abb. 45 zeigt den Unterschied zwischen den schweren Boxern und den leichten Boxern bei der Verwendung der neunzehn Finten. 


\subsection{Schwere Boxer verwenden Kombinierte Finten häufiger als leichte Boxer}

Boxer der sechs schwersten Gewichtsklassen (Weltergewicht, Halbmittelgewicht, Mittelgewicht, Halbschwergewicht, Schwergewicht und Superschwergewicht) verwenden Kombinierte Finten häufiger als Boxer der sechs leichtesten Gewichtsklassen (Halbfliegengewicht, Fliegengewicht, Bantamgewicht, Federgewicht, Leichtgewicht und Halbweltergewicht).

Tab 9: Boxer der sechs schwersten Gewichtsklassen $(n=28)$ verwenden Kombinierte Finten häufiger als Boxer der sechs leichtesten Gewichtsklassen $(n=28)$.

\begin{tabular}{|c|c|c|c|c|c|c|}
\hline $\begin{array}{l}\text { Kombinierte } \\
\text { Finten }\end{array}$ & $\begin{array}{l}\text { leichte } \\
\text { Boxer }\end{array}$ & $\begin{array}{c}\text { schwere } \\
\text { Boxer }\end{array}$ & di & Rangplatz & $d i<0$ & $d i>0$ \\
\hline ValBR & 173 & 270 & -97 & 7 & 7 & \\
\hline VaBR & 0 & 0 & 0 & 0 & & \\
\hline VarBR & 134 & 249 & -115 & 8 & 8 & \\
\hline KgSBAV & 340 & 410 & -70 & 6 & 6 & \\
\hline SSV & 250 & 280 & -30 & 5 & 5 & \\
\hline BSvBAV & 0 & 16 & -16 & 3 & 3 & \\
\hline KgSBAZ & 57 & 49 & 8 & 2 & & 2 \\
\hline SSZ & 25 & 3 & 22 & 4 & & 4 \\
\hline BSvBAZ & 0 & 5 & -5 & 1 & 1 & \\
\hline Summe & & & & 36 & $\mathrm{~T}=30$ & $T^{\prime}=6$ \\
\hline
\end{tabular}

Der T- Wert = 4 signifikant auf dem Niveau von 0,05 (Zweiseitig) Der T- Wert $=0$ signifikant auf dem Niveau von 0,01 (Zweiseitig) $\mathrm{T}=6>$ 4, d.h. das Ergebnis ist nicht signifikant.

In der Tab. 9 sind die Kombinierten Finten für die leichten Boxer und die Kombinierten Finten für die schweren Boxer aufgezeigt. Die Differenzen zwischen den Kombinierten Finten für die leichten Boxer und die schweren Boxer und die Rangplätze von di und di $<0$ oder di $>0$ sind ebenfalls dargestellt. 


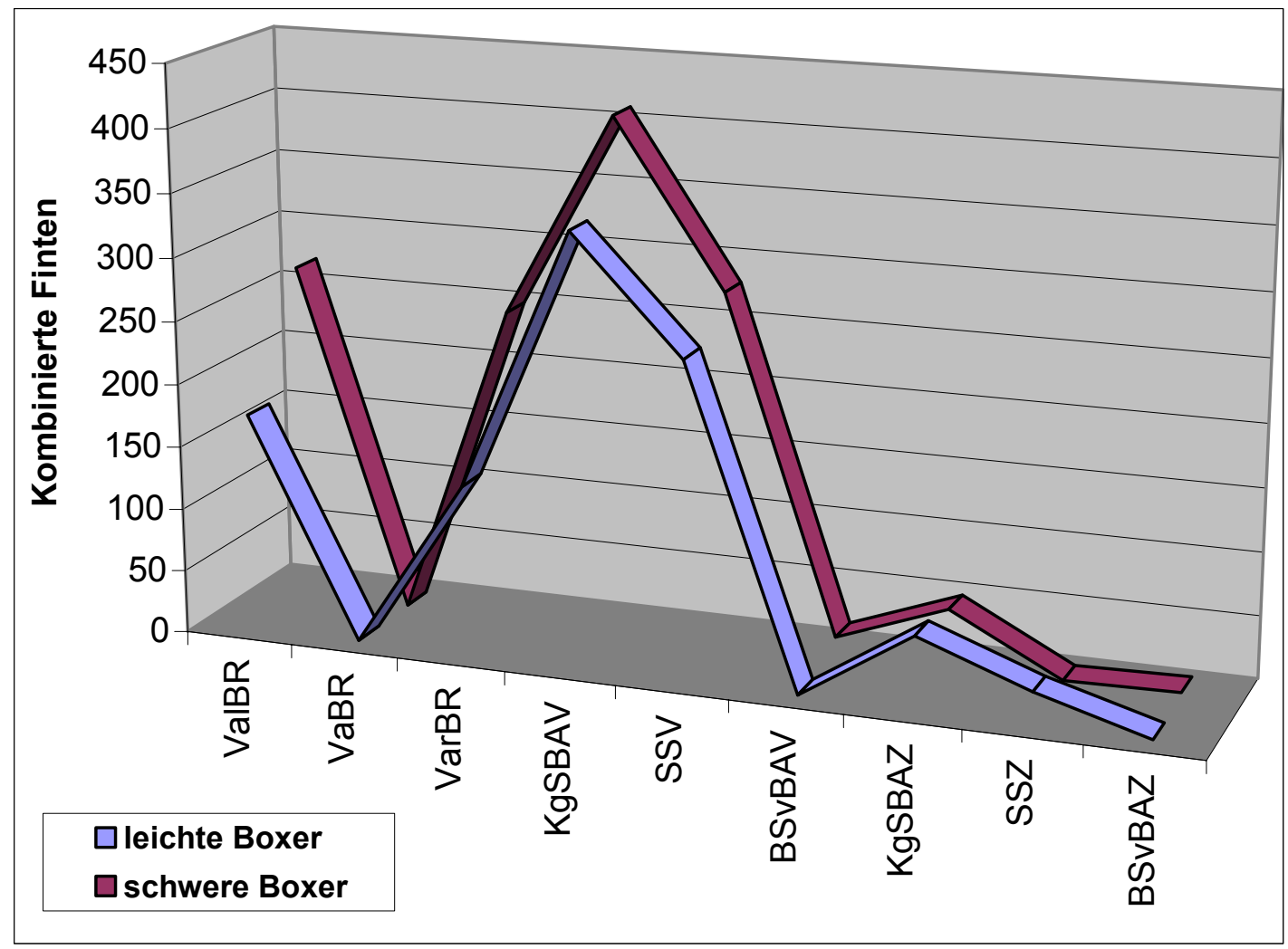

Abb. 46: Boxer der sechs schwersten Gewichtsklassen (Weltergewicht, Halbmittelgewicht, Mittelgewicht, Halbschwergewicht, Schwergewicht und Superschwergewicht) $(n=28)$ verwenden Kombinierte Finten häufiger als Boxer der sechs leichtesten Gewichtsklassen (Halbfliegengewicht, Fliegengewicht, Bantamgewicht, Federgewicht, Leichtgewicht und Halbweltergewicht) $(n=28)$.

Abb. 46 zeigt den Unterschied zwischen den schweren Boxern und den leichten Boxern bei der Verwendung der neun Kombinierten Finten. 
Boxer der fünf schwersten Gewichtsklassen (Halbmittelgewicht, Mittelgewicht, Halbschwergewicht, Schwergewicht und Superschwergewicht) verwenden Kombinierte Finten häufiger als Boxer der fünf leichtesten Gewichtsklassen (Halbfliegengewicht, Fliegengewicht, Bantamgewicht, Federgewicht und Leichtgewicht).

Tab 10: Boxer der fünf schwersten Gewichtsklassen $(n=26)$ verwenden Kombinierte Finten häufiger als Boxer der fünf leichtesten Gewichtsklas$\operatorname{sen}(n=26)$.

\begin{tabular}{|c|c|c|c|c|c|c|}
\hline $\begin{array}{c}\text { Kombinierte } \\
\text { Finten }\end{array}$ & $\begin{array}{l}\text { leichte } \\
\text { Boxer }\end{array}$ & $\begin{array}{c}\text { schwere } \\
\text { Boxer }\end{array}$ & di & Rangplatz & $d i<0$ & $d i>0$ \\
\hline ValBR & 154 & 251 & -97 & 7 & 7 & \\
\hline VaBR & 0 & 0 & 0 & 0 & & \\
\hline VarBR & 118 & 231 & -113 & 8 & 8 & \\
\hline KgSBAV & 314 & 382 & -68 & 6 & 6 & \\
\hline SSV & 228 & 263 & -35 & 5 & 5 & \\
\hline BSvBAV & 0 & 13 & -13 & 3 & 3 & \\
\hline KgSBAZ & 39 & 49 & -10 & 2 & 2 & \\
\hline SSZ & 19 & 3 & 16 & 4 & & 4 \\
\hline BSvBAZ & 0 & 5 & -5 & 1 & 1 & \\
\hline Summe & & & & 36 & $\mathrm{~T}=32$ & $\mathrm{~T}^{\prime}=4$ \\
\hline
\end{tabular}

Der T- Wert $=4$ signifikant auf dem Niveau von 0,05 (Zweiseitig) Der T- Wert $=0$ signifikant auf dem Niveau von 0,01 (Zweiseitig) $\mathrm{T}=4=4$, d.h. das Ergebnis ist signifikant auf dem 0,05 Niveau.

In der Tab. 10 sind die Kombinierten Finten für die leichten Boxer und die kombinierte Finten für die schweren Boxer aufgezeigt. Die Differenzen zwischen den Kombinierten Finten für die leichten Boxer und die schweren Boxer und die Rangplätze von di und di $<0$ oder di $>0$ sind ebenfalls dargestellt. 


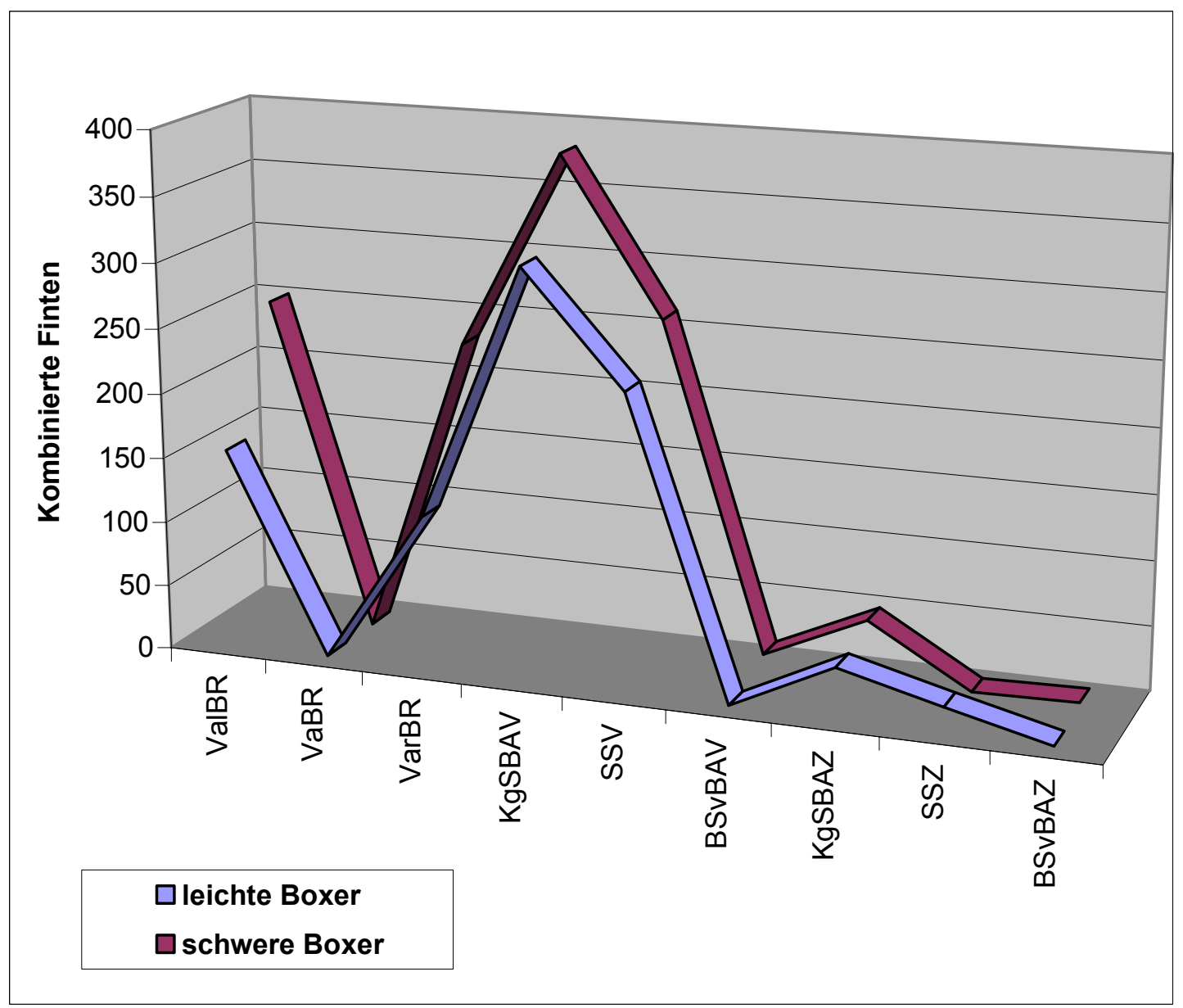

Abb. 47: Boxer der fünf schwersten Gewichtsklassen (Halbmittelgewicht, Mittelgewicht, Halbschwergewicht, Schwergewicht und Superschwergewicht) ( $n=26)$ verwenden Kombinierte Finten häufiger als Boxer der fünf leichtesten Gewichtsklassen (Halbfliegengewicht, Fliegengewicht, Bantamgewicht, Federgewicht und Leichtgewicht) $(n=26)$.

Abb. 47 zeigt den Unterschied zwischen den schweren Boxern und den leichten Boxern bei der Verwendung der neun Kombinierten Finten. 
Boxer der vier schwersten Gewichtsklassen (Mittelgewicht, Halbschwergewicht, Schwergewicht und Superschwergewicht) verwenden kombinierte Finten häufiger als Boxer der vier leichtesten Gewichtsklassen (Halbfliegengewicht, Fliegengewicht, Bantamgewicht und Federgewicht).

Tab 11: Boxer der vier schwersten Gewichtsklassen $(n=22)$ verwenden Kombinierte Finten häufiger als Boxer der vier leichtesten Gewichtsklas$\operatorname{sen}(n=22)$.

\begin{tabular}{|c|c|c|c|c|c|c|}
\hline $\begin{array}{c}\text { Kombinierte } \\
\text { Finten }\end{array}$ & $\begin{array}{c}\text { leichte } \\
\text { Boxer }\end{array}$ & $\begin{array}{c}\text { schwere } \\
\text { Boxer }\end{array}$ & di & Rangplatz & di<0 & di>0 \\
\hline ValBR & 133 & 209 & -76 & 7 & 7 & \\
\hline VaBR & 0 & 0 & 0 & 0 & 0 & \\
\hline VarBR & 103 & 192 & -89 & 8 & 8 & \\
\hline KgSBAV & 278 & 312 & -34 & 6 & 6 & \\
\hline SSV & 192 & 211 & -19 & 5 & 5 & \\
\hline BSvBAV & 0 & 12 & -12 & 3 & 3 & \\
\hline KgSBAZ & 37 & 48 & -11 & 2 & 2 & \\
\hline SSZ & 17 & 3 & 14 & 4 & & 4 \\
\hline BSvBAZ & 0 & 5 & -5 & 1 & 1 & \\
\hline Summe & & & & 36 & T=32 & $T^{\prime}=4$ \\
\hline
\end{tabular}

Der T- Wert $=4$ signifikant auf dem Niveau von 0,05 (Zweiseitig)

Der T- Wert $=0$ signifikant auf dem Niveau von 0,01 (Zweiseitig) $\mathrm{T}=4=4$, d.h. das Ergebnis ist signifikant auf dem 0,05 Niveau.

In der Tab. 11 sind die kombinierten Finten für die leichten Boxer und die kombinierte Finten für die schweren Boxer aufgezeigt. Die Differenzen zwischen den kombinierten Finten für die leichten Boxer und die schweren Boxer und die Rangplätze von di und di $<0$ oder di $>0$ sind ebenfalls dargestellt. 


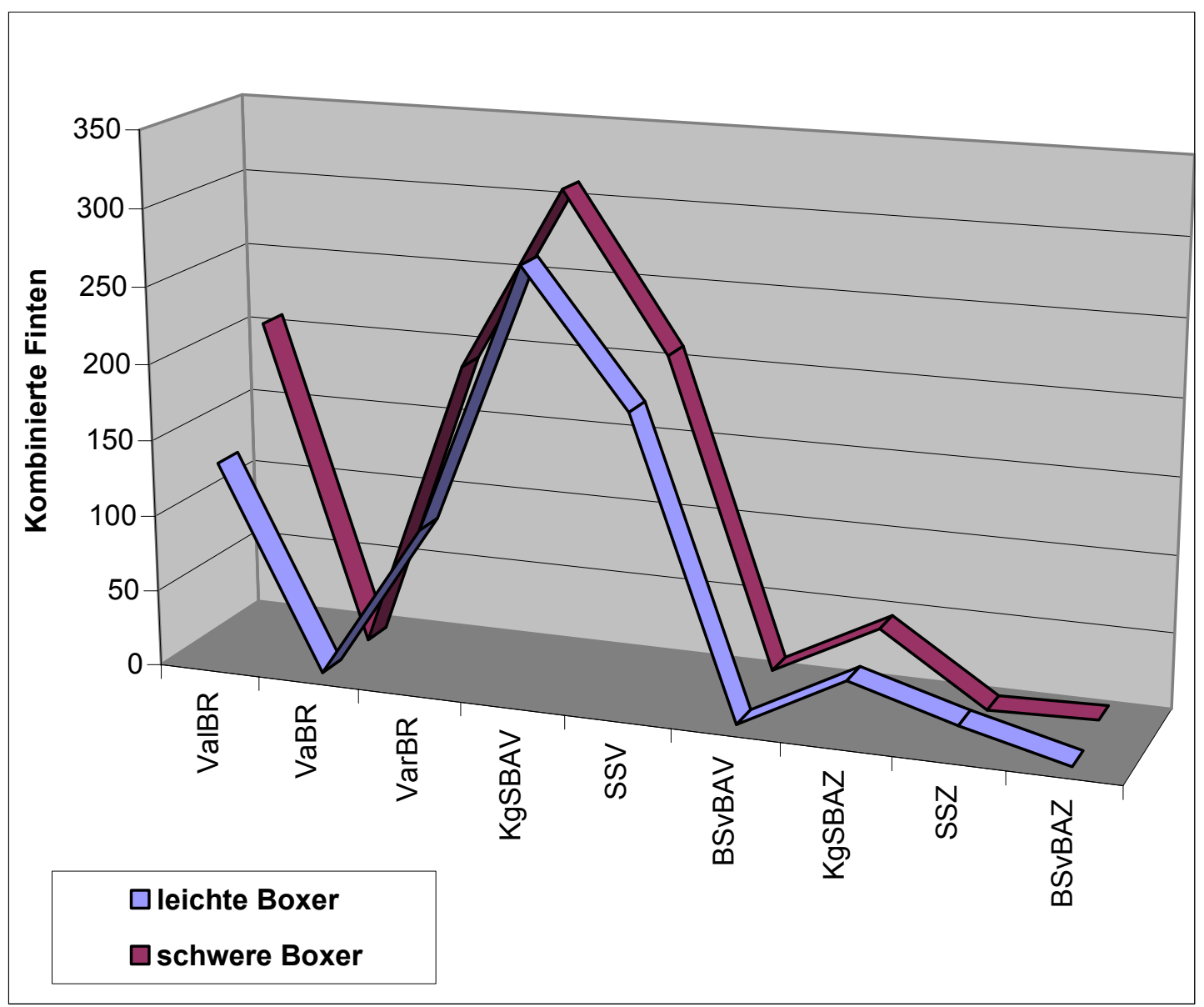

Abb. 48: Boxer der vier schwersten Gewichtsklassen (Mittelgewicht, Halbschwergewicht, Schwergewicht und Superschwergewicht) $(n=22)$ verwenden Kombinierte Finten häufiger als Boxer der vier leichtesten Gewichtsklassen (Halbfliegengewicht, Fliegengewicht, Bantamgewicht und Federgewicht) $(n=22)$.

Abb. 48 zeigt den Unterschied zwischen den schweren Boxern und den leichten Boxern bei der Verwendung der neun Kombinierten Finten. 
Boxer der drei schwersten Gewichtsklassen (Halbschwergewicht, Schwergewicht und Superschwergewicht) verwenden kombinierte Finten häufiger als Boxer der drei leichtesten Gewichtsklassen (Halbfliegengewicht, Fliegengewicht und Bantamgewicht).

Tab 12: Boxer der drei schwersten Gewichtsklassen $(n=18)$ verwenden Kombinierte Finten häufiger als Boxer der drei leichtesten Gewichtsklas$\operatorname{sen}(n=18)$.

\begin{tabular}{|c|c|c|c|c|c|c|}
\hline $\begin{array}{l}\text { Kombinierte } \\
\text { Finten }\end{array}$ & $\begin{array}{l}\text { leichte } \\
\text { Boxer }\end{array}$ & $\begin{array}{c}\text { schwere } \\
\text { Boxer }\end{array}$ & di & Rangplatz & $d i<0$ & $d i>0$ \\
\hline ValBR & 118 & 154 & -36 & 7 & 7 & \\
\hline VaBR & 0 & 0 & 0 & 0 & & \\
\hline VarBR & 89 & 124 & -35 & 6 & 6 & \\
\hline KgSBAV & 198 & 253 & -55 & 8 & 8 & \\
\hline SSV & 142 & 125 & 17 & 5 & & 5 \\
\hline BSvBAV & 0 & 8 & -8 & 2 & 2 & \\
\hline KgSBAZ & 32 & 43 & -11 & 4 & 4 & \\
\hline SSZ & 12 & 3 & 9 & 3 & & 3 \\
\hline BSvBAZ & 0 & 5 & -5 & 1 & 1 & \\
\hline Summe & & & & 36 & $\mathrm{~T}=28$ & $\mathrm{~T}^{\prime}=8$ \\
\hline
\end{tabular}

Der T- Wert $=4$ signifikant auf dem Niveau von 0,05 (Zweiseitig)

Der T- Wert $=0$ signifikant auf dem Niveau von 0,01 (Zweiseitig)

$\mathrm{T}=8>4$, d.h. das Ergebnis ist nicht signifikant.

In der Tab. 12 sind die Kombinierten Finten für die leichten Boxer und die kombinierten Finten für die schweren Boxer aufgezeigt. Die Differenzen zwischen den Kombinierten Finten für die leichten Boxer und die schweren Boxer und die Rangplätze von di und di $<0$ oder di $>0$ sind ebenfalls dargestellt. 


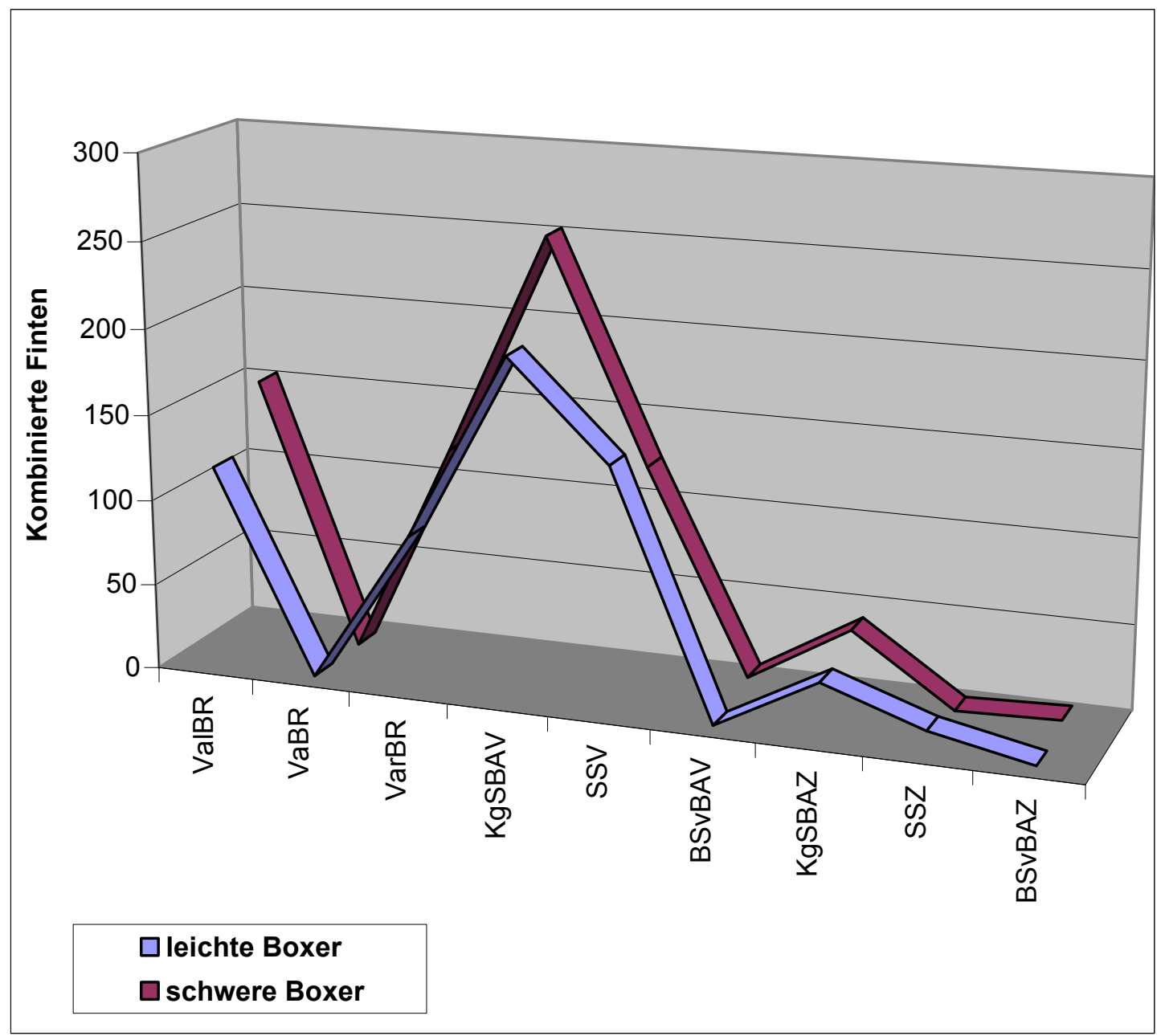

Abb. 49: Boxer der drei schwersten Gewichtsklassen (Halbschwergewicht, Schwergewicht und Superschwergewicht) $(n=18)$ verwenden Kombinierte Finten häufiger als Boxer der drei leichtesten Gewichtsklassen (Halbfliegengewicht, Fliegengewicht und Bantamgewicht) $(n=18)$.

Abb. 49 zeigt den Unterschied zwischen den schweren Boxern und den leichten Boxern bei der Verwendung der neun Kombinierten Finten. 


\subsection{Der Sieger macht gegen Ende des Kampfes häufiger vari- able Finten als der Verlierer}

Tabelle 13 zeigt den Unterschied zwischen dem Sieger und dem Verlierer bei der Verwendung der variablen Finten in den Runden 1+2 sowie 3+4.

Tab 13: Der Sieger $(n=28)$ macht gegen Ende des Kampfes häufiger variable Finten als der Verlierer $(n=28)$.

\begin{tabular}{|cccc|}
\hline & Sieger & Verlierer & Summe \\
\hline Finten in Rd 1+2 & 535 & 472 & 1007 \\
\hline Finten in Rd 3+4 & 486 & 401 & 887 \\
\hline Summe & 1021 & 873 & 1894 \\
\hline
\end{tabular}

\begin{tabular}{|c|c|c|c|c|c|}
\hline & $\mathbf{F b}$ & $\mathrm{Fe}$ & $(\mathrm{Fb}-\mathrm{Fe})$ & $(\mathrm{Fb}-\mathrm{Fe})^{2}$ & $\frac{(\mathrm{Fb}-\mathrm{Fe})^{2}}{\mathrm{Fe}}$ \\
\hline $\begin{array}{l}\text { Finten in } \\
\text { Rd1+2 von Sieger }\end{array}$ & 535 & 542,8 & $-7,8$ & 60,84 & 0,11 \\
\hline $\begin{array}{l}\text { Finten in } \\
\text { Rd1+2 von Verlierer }\end{array}$ & 472 & 464,2 & 7,8 & 60,84 & 0,13 \\
\hline $\begin{array}{l}\text { Finten in } \\
\text { Rd3+4 von Sieger }\end{array}$ & 486 & 478,2 & 7,8 & 60,84 & 0,13 \\
\hline $\begin{array}{l}\text { Finten in } \\
\text { Rd3+4 von Verlierer }\end{array}$ & 401 & 408,8 & $-7,8$ & 60,84 & 0,15 \\
\hline Summe & 1894 & 1894 & 0 & & $x^{2}=0,52$ \\
\hline
\end{tabular}

$\mathrm{Fb}=$ Messwert

$\mathrm{Fe}=$ erwarteter Messwert bei Gleichverteilung

Der $X^{2}$ - Wert $=3,84$ signifikant auf dem Niveau von 0,05 (Zweiseitig)

Der $X^{2}$ - Wert $=10,8$ signifikant auf dem Niveau von 0,01 (Zweiseitig)

$X^{2}=0,52<3,84$, d.h. das Ergebnis ist nicht signifikant.

In der Tabelle 13 sind die zu erwartenden Häufigkeiten der variablen Finten nach der $\mathbf{X}^{\mathbf{2}}$ - Test Methode dargestellt. 


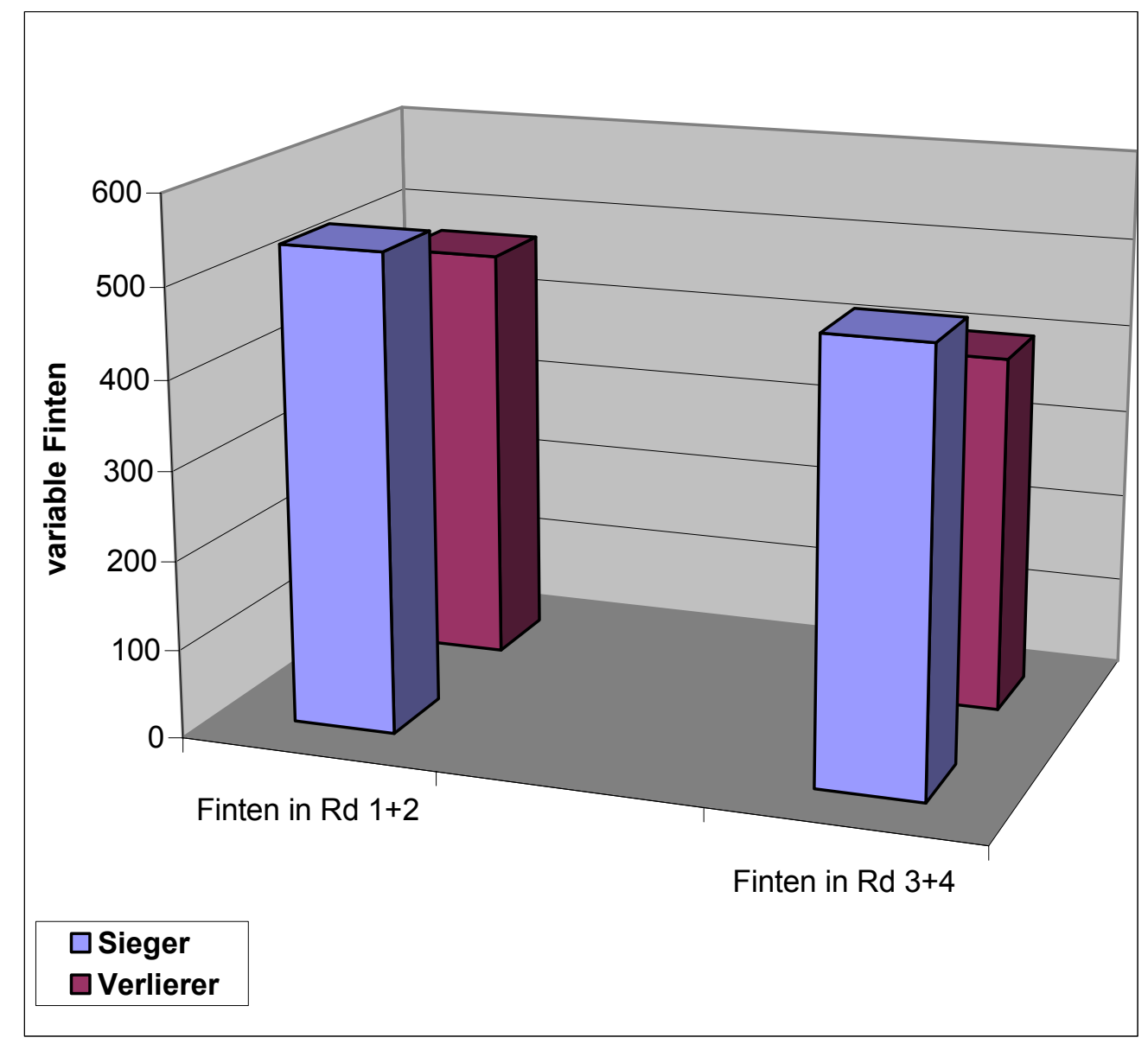

Abb. 50: Unterschiede zwischen den Siegern $(n=28)$ und den Verlierern $(n=28)$ bei variablen Finten in Abhängigkeit zur Kampfdauer.

Abb. 50 zeigt, dass der Sieger gegen Ende des Kampfes häufiger variable Finten macht, aber die Unterschiede zu dem Verlierer sind statistisch nicht signifikant. 


\subsubsection{Schwere Sieger machen gegen Ende des Kampfes häufi- ger variable Finten als leichte Sieger}

Tabelle 14 zeigt den Unterschied zwischen dem schweren Sieger und dem leichten Sieger bei der Verwendung der variablen Finten in den Runden 1+2 sowie $3+4$.

Tab 14: Schwere Sieger $(n=14)$ machen gegen Ende des Kampfes häufiger variable Finten als leichte Sieger $(n=14)$.

\begin{tabular}{|c|ccc|}
\hline & Schwere Sieger & leichte Sieger & Summe \\
\hline Finten in Rd 1+2 & 273 & 262 & 535 \\
\hline Finten in Rd 3+4 & 270 & 216 & 486 \\
\hline Summe & 543 & 478 & 1021 \\
\hline
\end{tabular}

\begin{tabular}{|c|c|c|c|c|c|}
\hline & $\mathbf{F b}$ & $\mathrm{Fe}$ & $(\mathrm{Fb}-\mathrm{Fe})$ & $(\mathrm{Fb}-\mathrm{Fe})^{2}$ & $\frac{(\mathrm{Fb}-\mathrm{Fe})^{2}}{\mathrm{Fe}}$ \\
\hline $\begin{array}{l}\text { Finten in } \\
\text { Rd1+2 von Schwere }\end{array}$ & 273 & 284,53 & $-11,53$ & 132,94 & 0,47 \\
\hline $\begin{array}{l}\text { Finten in } \\
\text { Rd1+2 von leichte }\end{array}$ & 262 & 250,47 & 11,53 & 132,94 & 0,53 \\
\hline $\begin{array}{l}\text { Finten in } \\
\text { Rd3+4 von Schwere }\end{array}$ & 270 & 258,47 & 11,53 & 132,94 & 0,51 \\
\hline $\begin{array}{l}\text { Finten in } \\
\text { Rd3+4 von leichte }\end{array}$ & 216 & 227,53 & $-11,53$ & 132,94 & 0,58 \\
\hline Summe & 1021 & 1021 & 0 & & $x^{2}=2,09$ \\
\hline
\end{tabular}

$\mathrm{Fb}=$ Messwert

$\mathrm{Fe}=$ erwarteter Messwert bei Gleichverteilung

Der $X^{2}$ - Wert $=3,84$ signifikant auf dem Niveau von 0,05 (Zweiseitig)

Der $X^{2}$ - Wert $=10,8$ signifikant auf dem Niveau von 0,01 (Zweiseitig)

$X^{2}=2,09<3,84$, d.h. das Ergebnis ist nicht signifikant.

In der Tabelle 14 sind die zu erwartenden Häufigkeiten der variablen Finten nach der $\mathbf{X}^{\mathbf{2}}$ - Test Methode dargestellt. 


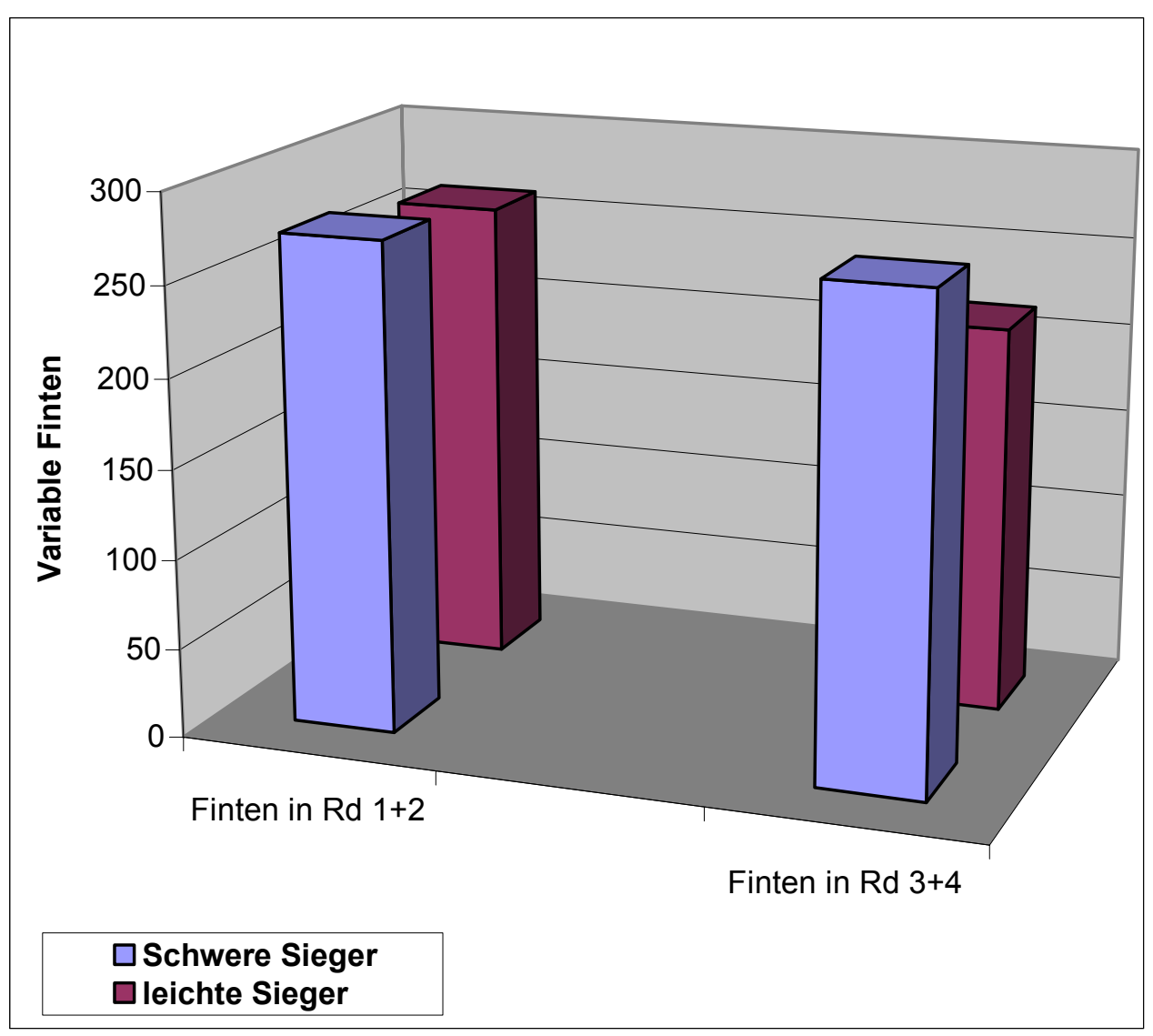

Abb. 51: Unterschiede zwischen den schweren Siegern $(n=14)$ und den leichten Siegern $(n=14)$ bei variablen Finten in Abhängigkeit zur Kampfdauer.

Abb. 51 zeigt, dass der schwere Sieger gegen Ende des Kampfes häufiger variable Finten macht, die Unterschiede sind statistisch nicht signifikant. 


\subsubsection{Schwere Boxer machen gegen Ende des Kampfes häufi- ger variable Finten als leichte Boxer}

Tabelle 15 zeigt den Unterschied zwischen dem schweren Boxer und dem leichten Boxer bei der Verwendung der variablen Finten in den Runden 1+2 sowie $3+4$.

Tab 15: Schwere Boxer $(n=28)$ machen gegen Ende des Kampfes häufiger variable Finten als leichte Boxer $(n=28)$.

\begin{tabular}{|c|ccc|}
\hline & Schwere Boxer & leichte Boxer & Summe \\
\hline Finten in Rd 1+2 & 528 & 479 & 1007 \\
\hline Finten in Rd 3+4 & 493 & 394 & 887 \\
\hline Summe & 1021 & 873 & 1894 \\
\hline
\end{tabular}

\begin{tabular}{|c|c|c|c|c|c|}
\hline & $\mathbf{F b}$ & $\mathrm{Fe}$ & $(\mathrm{Fb}-\mathrm{Fe})$ & $(\mathrm{Fb}-\mathrm{Fe})^{2}$ & $\frac{(\mathrm{Fb}-\mathrm{Fe})^{2}}{\mathrm{Fe}}$ \\
\hline $\begin{array}{l}\text { Finten in } \\
\text { Rd1+2 von Schwere }\end{array}$ & 528 & 542,8 & $-14,8$ & 219,04 & 0,40 \\
\hline $\begin{array}{l}\text { Finten in } \\
\text { Rd1+2 von leichte }\end{array}$ & 479 & 464,2 & 14,8 & 219,04 & 0,47 \\
\hline $\begin{array}{l}\text { Finten in } \\
\text { Rd3+4 von Schwere }\end{array}$ & 493 & 478,2 & 14,8 & 219,04 & 0.46 \\
\hline $\begin{array}{l}\text { Finten in } \\
\text { Rd3+4 von leichte }\end{array}$ & 394 & 408,8 & $-14,8$ & 219,04 & 0,54 \\
\hline Summe & 1894 & 1894 & 0 & & $x^{2}=1,87$ \\
\hline
\end{tabular}

$\mathrm{Fb}=$ Messwert

$\mathrm{Fe}=$ erwarteter Messwert bei Gleichverteilung

Der $X^{2}$ - Wert $=3,84$ signifikant auf dem Niveau von 0,05 (Zweiseitig)

Der $X^{2}$ - Wert $=10,8$ signifikant auf dem Niveau von 0,01 (Zweiseitig)

$X^{2}=1,87<3,84$, d.h. das Ergebnis ist nicht signifikant.

In der Tabelle 15 sind die zu erwartenden Häufigkeiten der variablen Finten nach der $\mathbf{X}^{\mathbf{2}}$ - Test Methode dargestellt. 


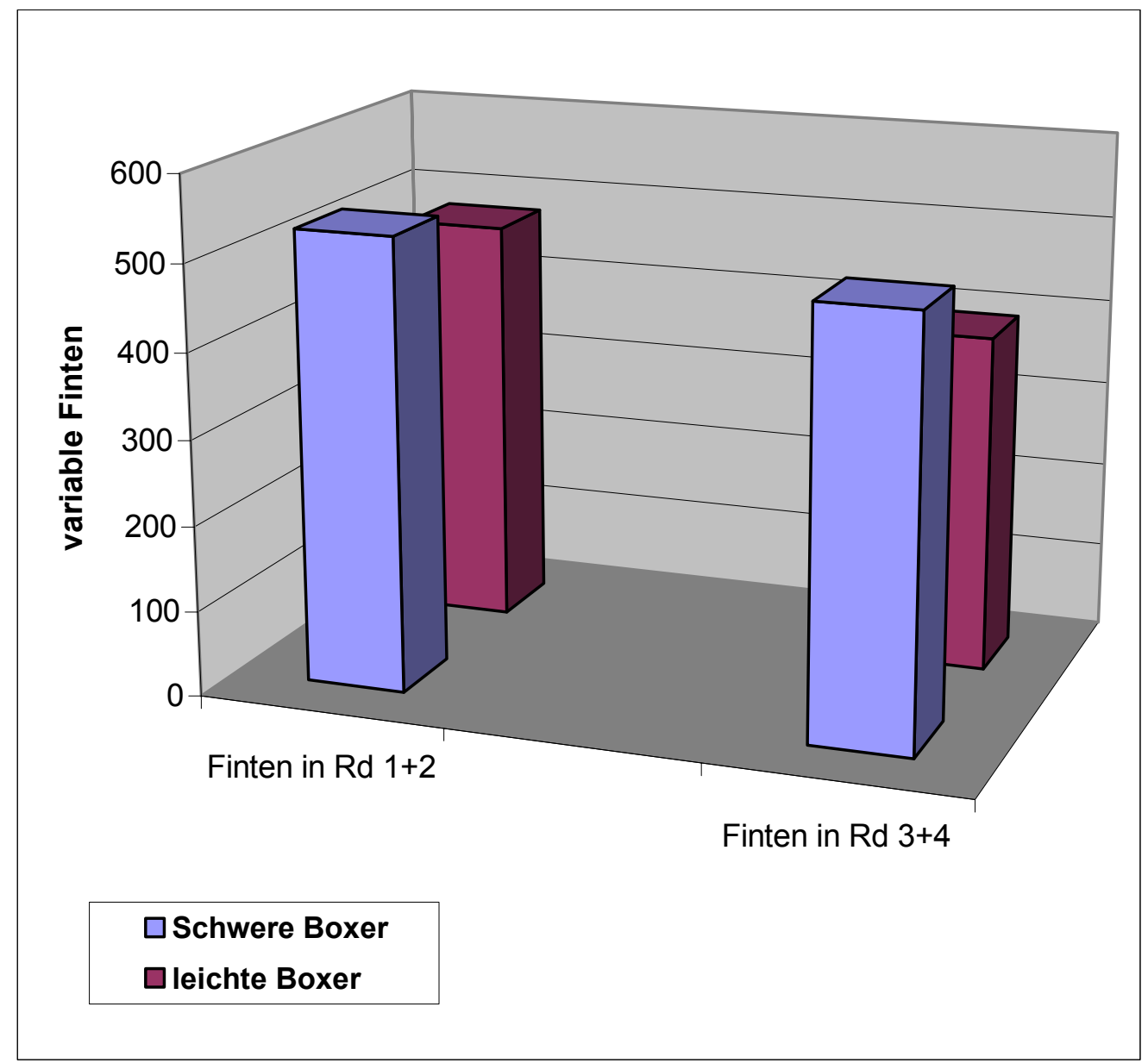

Abb. 52: Unterschiede zwischen den schweren Siegern $(n=28)$ und den leichten Siegern $(n=28)$ bei variablen Finten in Abhängigkeit zur Kampfdauer.

Die Abb. 52 zeigt, dass der schwere Boxer gegen Ende des Kampfes häufiger variable Finten macht, die Unterschiede sind statistisch nicht signifikant. 


\subsection{Die Sieger variieren die Art der Finten von Runde zu Run- de stärker als die Verlierer}

Tabelle 16 zeigt den Unterschied zwischen den Siegern und den Verlierern bei der Verwendung der neunzehn Finten in der ersten Runde sowie die Signifikanzen aus dem Wilcoxon-Test.

Tab 16: In der ersten Runde variiert der Sieger $(n=28)$ die Art der Finten stärker als die Verlierer $(n=28)$.

\begin{tabular}{|c|c|c|c|c|c|c|}
\hline Finten & $\begin{array}{c}\text { Sieger in } \\
\text { Rd1 }\end{array}$ & $\begin{array}{l}\text { Verlierer in } \\
\text { Rd1 }\end{array}$ & di & Rangplatz & $d i<0$ & $d i>0$ \\
\hline HV & 87 & 52 & 35 & 12 & & 12 \\
\hline HR & 63 & 30 & 33 & 11 & & 11 \\
\hline$A b$ & 49 & 31 & 18 & 8,5 & & 8,5 \\
\hline ValR & 73 & 45 & 28 & 10 & & 10 \\
\hline VaR & 0 & 1 & -1 & 1,5 & 1,5 & \\
\hline VarR & 58 & 40 & 18 & 8,5 & & 8,5 \\
\hline KFi & 51 & 36 & 15 & 6 & & 6 \\
\hline $\mathrm{KFa}$ & 10 & 9 & 1 & 1,5 & & 1,5 \\
\hline KgSA & 115 & 69 & 46 & 13 & & 13 \\
\hline BSvA & 18 & 8 & 10 & 5 & & 5 \\
\hline ValBR & 95 & 31 & 64 & 15 & & 15 \\
\hline VaBR & 0 & 0 & 0 & 0 & & \\
\hline VarBR & 82 & 33 & 49 & 13 & & 13 \\
\hline KgSBAV & 141 & 74 & 67 & 16 & & 16 \\
\hline SSV & 108 & 50 & 58 & 14 & & 14 \\
\hline BSvBAV & 7 & 4 & 3 & 3 & & 3 \\
\hline KgSBAZ & 27 & 10 & 17 & 7 & & 7 \\
\hline SSZ & 6 & 1 & 5 & 4 & & 4 \\
\hline BSvBAZ & 0 & 0 & 0 & 0 & & \\
\hline Summe & & & & 149 & $\mathrm{~T}=1,5$ & $T^{\prime}=147,5$ \\
\hline
\end{tabular}

Der T- Wert $=35$ signifikant auf dem Niveau von 0,05 (Zweiseitig)

Der T- Wert $=23$ signifikant auf dem Niveau von 0,01 (Zweiseitig) $T=1,5<23$, d.h. das Ergebnis ist hoch signifikant auf dem 0,01 Niveau.

In der Tab. 16 sind die Finten in der ersten Runde für die Sieger und die Finten für die Verlierer aufgezeigt. Die Differenzen zwischen den Finten der Sieger und den Finten der Verlierer und die Rangplätze von di und di $<0$ oder di $>0$ sind ebenfalls dargestellt. 


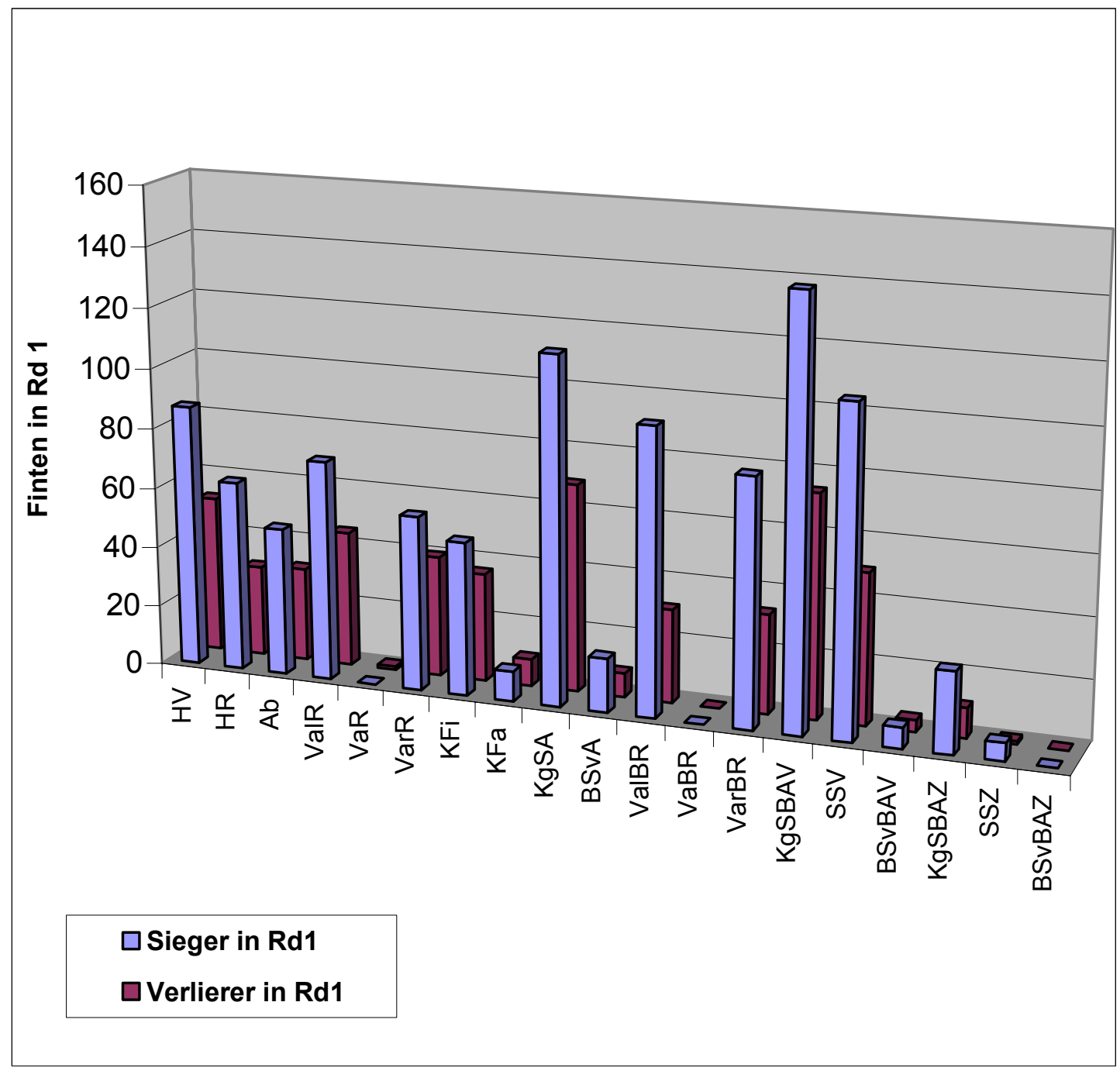

Abb. 53: In der ersten Runde variiert der Sieger $(n=28)$ die Art der Finten stärker als die Verlierer $(n=28)$.

Abb. 53 zeigt den Unterschied zwischen den Siegern und den Verlierern bei der Verwendung der neunzehn Finten in der ersten Runde. 
Tabelle 17 zeigt den Unterschied zwischen den Siegern und den Verlierern bei der Verwendung der neunzehn Finten in der zweiten Runde sowie die Signifikanzen aus dem Wilcoxon-Test.

Tab 17: In der zweiten Runde variiert der Sieger $(n=28)$ die Art der Finten stärker als der Verlierer $(\mathbf{n}=\mathbf{2 8})$.

\begin{tabular}{|c|c|c|c|c|c|c|}
\hline Finten & $\begin{array}{l}\text { Sieger in } \\
\text { Rd2 }\end{array}$ & $\begin{array}{l}\text { Verlierer in } \\
\text { Rd2 }\end{array}$ & di & Rangplatz & $d i<0$ & $d i>0$ \\
\hline HV & 76 & 49 & 27 & 9 & & 9 \\
\hline HR & 61 & 31 & 30 & 11 & & 11 \\
\hline$A b$ & 28 & 26 & 2 & 2,5 & & 2,5 \\
\hline ValR & 66 & 37 & 29 & 10 & & 10 \\
\hline VaR & 0 & 0 & 0 & 0 & & \\
\hline VarR & 49 & 27 & 22 & 8 & & 8 \\
\hline $\mathrm{KFi}$ & 35 & 27 & 8 & 6 & & 6 \\
\hline KFa & 13 & 15 & -2 & 2,5 & 2,5 & \\
\hline KgSA & 102 & 59 & 43 & 12 & & 12 \\
\hline BSvA & 17 & 18 & -1 & 1 & 1 & \\
\hline ValBR & 89 & 32 & 57 & 15 & & 15 \\
\hline VaBR & 0 & 0 & 0 & 0 & & \\
\hline VarBR & 75 & 28 & 47 & 13,5 & & 13,5 \\
\hline KgSBAV & 133 & 55 & 78 & 16 & & 16 \\
\hline SSV & 89 & 42 & 47 & 13,5 & & 13,5 \\
\hline BSvBAV & 0 & 0 & 0 & 0 & & \\
\hline KgSBAZ & 23 & 6 & 17 & 7 & & 7 \\
\hline SSZ & 6 & 0 & 6 & 5 & & 5 \\
\hline BSvBAZ & 5 & 0 & 5 & 4 & & 4 \\
\hline Summe & & & & 136 & $\mathrm{~T}=3,5$ & $T^{\prime}=132,5$ \\
\hline
\end{tabular}

Der T- Wert $=30$ signifikant auf dem Niveau von 0,05 (Zweiseitig)

Der T- Wert $=20$ signifikant auf dem Niveau von 0,01 (Zweiseitig)

$T=3,5<20$, d. h. das Ergebnis ist hoch signifikant auf dem 0,01 Niveau.

In der Tab. 17 sind die Finten der zweiten Runde für die Sieger und die Finten für die Verlierer aufgezeigt. Die Differenzen zwischen den Finten der Sieger und den Finten der Verlierer und die Rangplätze von di und di $<0$ oder di $>0$ sind ebenfalls dargestellt. 


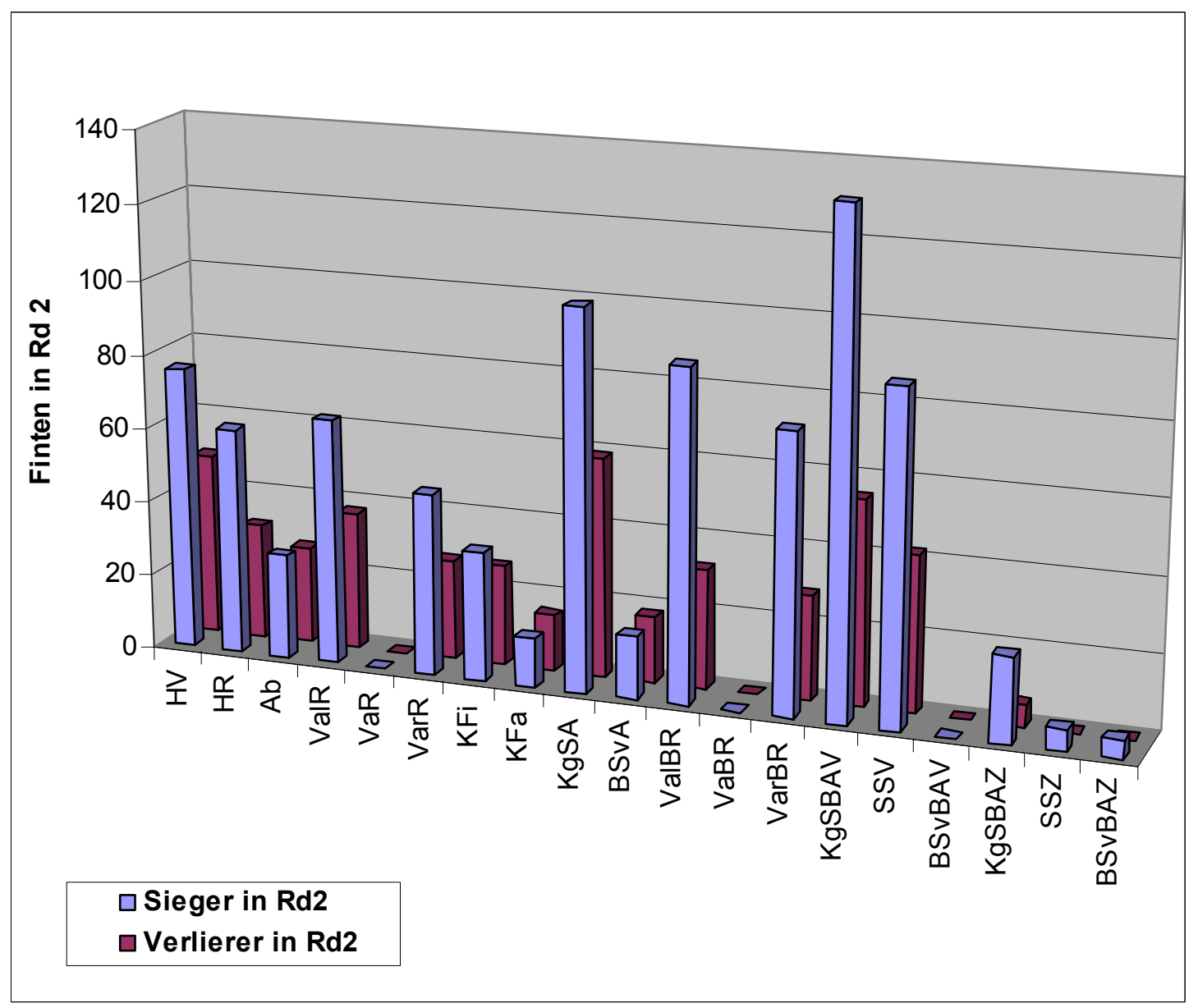

Abb. 54: In der zweiten Runde variiert der Sieger $(n=28)$ die Art der Finten stärker als der Verlierer $(n=28)$.

Abb. 54 zeigt den Unterschied zwischen den Siegern und den Verlierern bei der Verwendung der neunzehn Finten in der zweiten Runde. 
Tabelle 18 zeigt den Unterschied zwischen den Siegern und den Verlierern bei der Verwendung der neunzehn Finten in der dritten Runde sowie die Signifikanzen aus dem Wilcoxon-Test.

Tab 18: In der dritten Runde variiert der Sieger $(n=28)$ die Art der Finten stärker als der Verlierer $(\mathbf{n}=\mathbf{2 8})$.

\begin{tabular}{|c|c|c|c|c|c|c|}
\hline Finten & Sieger in Rd3 & Verlierer in Rd3 & di & Rangplatz & $d i<0$ & $d i>0$ \\
\hline HV & 72 & 51 & 21 & 9,5 & & 9,5 \\
\hline HR & 53 & 32 & 21 & 9,5 & & 9,5 \\
\hline$A b$ & 41 & 22 & 19 & 7 & & 7 \\
\hline ValR & 55 & 36 & 19 & 7 & & 7 \\
\hline VaR & 0 & 0 & 0 & 0 & & 0 \\
\hline VarR & 47 & 28 & 19 & 7 & & 7 \\
\hline $\mathrm{KFi}$ & 37 & 19 & 18 & 5 & & 5 \\
\hline $\mathrm{KFa}$ & 5 & 5 & 0 & 0 & & 0 \\
\hline $\mathrm{KgSA}$ & 94 & 46 & 48 & 14 & & 14 \\
\hline BSvA & 17 & 7 & 10 & 3 & & 3 \\
\hline ValBR & 69 & 31 & 38 & 11,5 & & 11,5 \\
\hline VaBR & 0 & 0 & 0 & 0 & & 0 \\
\hline VarBR & 59 & 21 & 38 & 11,5 & & 11,5 \\
\hline KgSBAV & 125 & 63 & 62 & 15 & & 15 \\
\hline SSV & 87 & 45 & 42 & 13 & & 13 \\
\hline BSvBAV & 3 & 0 & 3 & 1 & & 1 \\
\hline KgSBAZ & 17 & 3 & 14 & 4 & & 4 \\
\hline SSZ & 5 & 1 & 4 & 2 & & 2 \\
\hline BSvBAZ & 0 & 0 & 0 & 0 & & \\
\hline Summe & & & & 120 & $\mathrm{~T}=0$ & $T^{\prime}=120$ \\
\hline
\end{tabular}

Der T- Wert $=46$ signifikant auf dem Niveau von 0,05 (Zweiseitig) Der T- Wert $=32$ signifikant auf dem Niveau von 0,01 (Zweiseitig) $\mathrm{T}=0<20$, d. h. das Ergebnis ist hoch signifikant auf dem 0,01 Niveau.

In der Tab. 18 sind die Finten der dritten Runde für die Sieger und die Finten der Verlierer aufgezeigt. Die Differenzen zwischen den Finten für die Sieger und die Verlierer und die Rangplätze von di und di $<0$ oder di $>0$ sind ebenfalls dargestellt. 


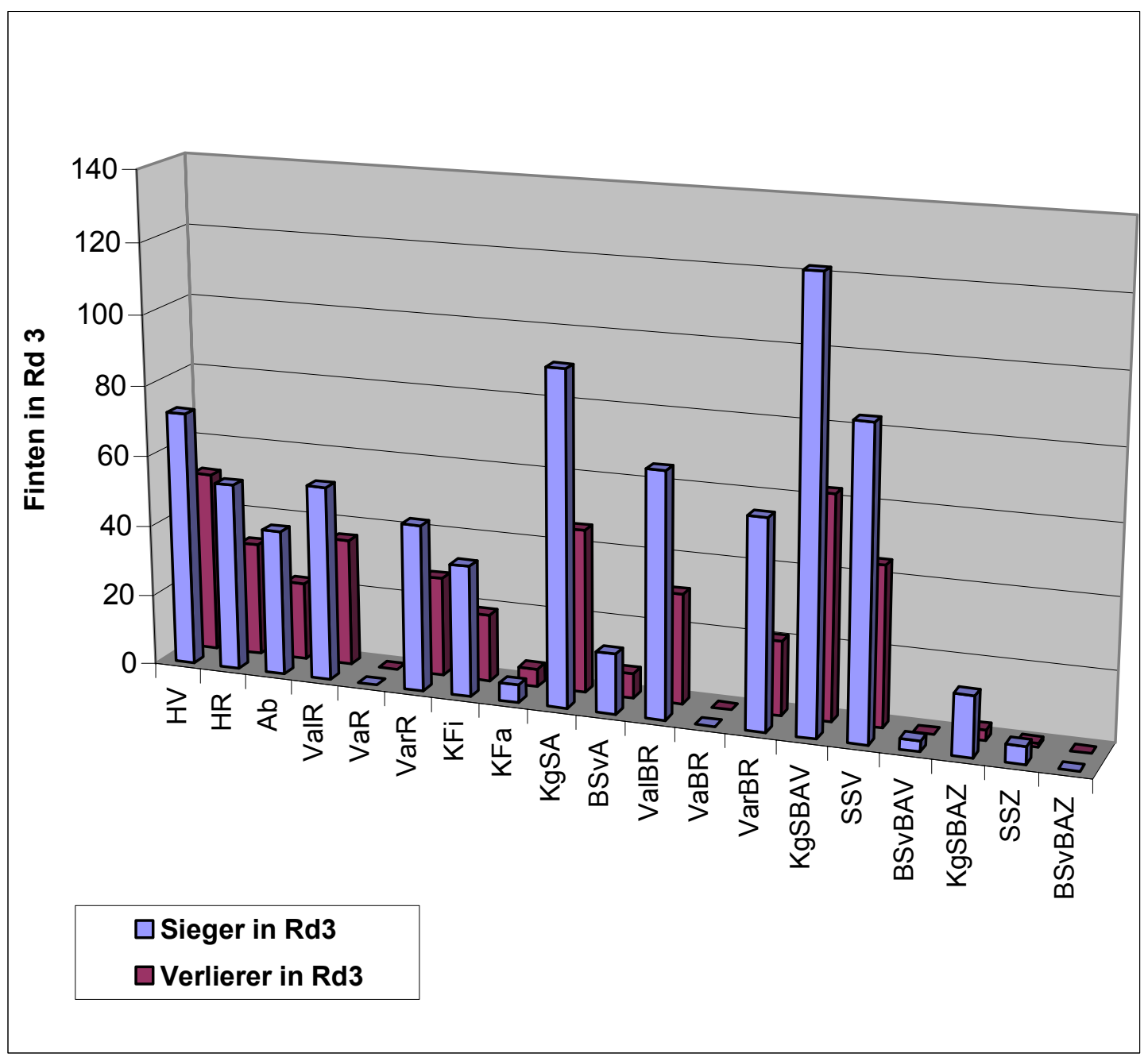

Abb. 55: In der dritten Runde variiert der Sieger $(n=28)$ die Art der Finten stärker als der Verlierer $(n=28)$.

Abb. 55 zeigt den Unterschied zwischen den Siegern und den Verlierern bei der Verwendung der neunzehn Finten in der dritten Runde. 
Tabelle 19 zeigt den Unterschied zwischen den Siegern und den Verlierern bei der Verwendung der neunzehn Finten in der vierten Runde sowie die Signifikanzen aus dem Wilcoxon-Test.

Tab 19: In der vierten Runde variiert der Sieger $(n=28)$ die Art der Finten stärker als die Verlierer $(n=28)$.

\begin{tabular}{|c|c|c|c|c|c|c|}
\hline Finten & $\begin{array}{l}\text { Sieger in } \\
\text { Rd44 }\end{array}$ & $\begin{array}{c}\text { Verlierer in } \\
\text { Rd4 }\end{array}$ & di & Rangplatz & $d i<0$ & $d i>0$ \\
\hline HV & 58 & 48 & 10 & 8 & & 8 \\
\hline HR & 45 & 27 & 18 & 10 & & 10 \\
\hline$A b$ & 39 & 22 & 17 & 9 & & 9 \\
\hline ValR & 56 & 20 & 36 & 13,5 & & 13,5 \\
\hline VaR & 4 & 0 & 4 & 7 & & 7 \\
\hline VarR & 56 & 19 & 37 & 15 & & 15 \\
\hline KFi & 18 & 19 & -1 & 2,5 & 2,5 & \\
\hline KFa & 5 & 3 & 2 & 5,5 & & 5,5 \\
\hline KgSA & 72 & 36 & 36 & 13,5 & & 13,5 \\
\hline BSvA & 4 & 5 & -1 & 2,5 & 2,5 & \\
\hline ValBR & 67 & 26 & 41 & 16 & & 16 \\
\hline VaBR & 0 & 0 & 0 & 0 & & \\
\hline VarBR & 57 & 23 & 34 & 12 & & 12 \\
\hline KgSBAV & 104 & 53 & 51 & 17 & & 17 \\
\hline SSV & 67 & 43 & 24 & 11 & & 11 \\
\hline BSvBAV & 2 & 0 & 2 & 5,5 & & 5,5 \\
\hline KgSBAZ & 11 & 12 & -1 & 2,5 & 2,5 & \\
\hline SSZ & 4 & 3 & 1 & 1,5 & & 2,5 \\
\hline BSvBAZ & 0 & 0 & 0 & 0 & & \\
\hline Summe & & & & 153 & $\mathrm{~T}=7,5$ & $T^{\prime}=145,5$ \\
\hline
\end{tabular}

Der T- Wert $=35$ signifikant auf dem Niveau von 0,05 (Zweiseitig)

Der T- Wert $=23$ signifikant auf dem Niveau von 0,01 (Zweiseitig) $T=7,5<23, d$. h. das Ergebnis ist hoch signifikant auf dem 0,01 Niveau.

In der Tab. 19 sind die Finten in der vierten Runde für die Sieger und die Finten der Verlierer aufgezeigt. Die Differenzen zwischen den Finten der Sieger und den Finten der Verlierer und die Rangplätze von di und di $<0$ oder di $>0$ sind ebenfalls dargestellt. 


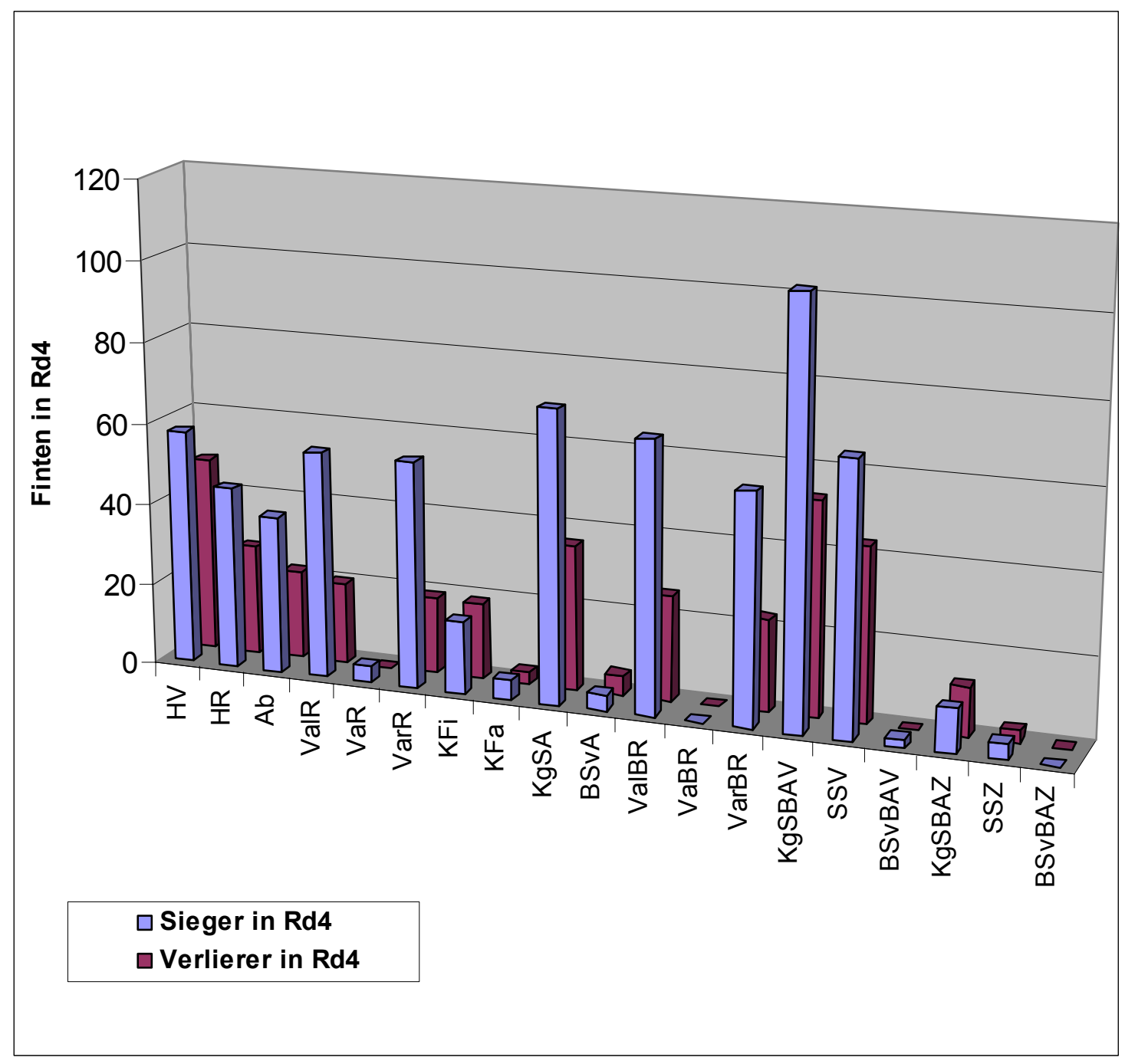

Abb. 56: In der vierten Runde variiert der Sieger $(n=28)$ die Art der Finten stärker als die Verlierer $(n=28)$.

Abb. 56 zeigt den Unterschied zwischen den Siegern und den Verlierern bei der Verwendung der neunzehn Finten in der vierten Runde. 


\subsection{Nationale Boxschulen}

Es gibt erfolgreiche nationale Boxschulen, die sich mit eigenem Fintierstil darstellen und somit auch mit unterschiedlichen Fintiermustern arbeiten. Anhand der Medaillenhäufigkeit (siehe Anhang 9) und des mir zu Verfügung stehenden Medienmaterials (Video-Aufzeichnungen) habe ich die amerikanische, russische, kubanische, ukrainische und die kasachische Boxschule ausgewählt und deren Fintierarten nachfolgend in Unterschied und Effizienz aufgelistet. Die Fintierstile der anderen Nationen habe ich nicht ausgewählt, da es nicht genügend Kämpfer in den Halbfinal- und Finalkämpfen gab, um sinnvoll von "Schulen" sprechen zu können.

\subsubsection{Die amerikanische Boxschule unterscheidet sich von de- nen aus Russland, der Ukraine, Kuba und Kasachstan}

Tabelle 20 zeigt den Unterschied zwischen den Boxern aus den USA und den Boxern aus Russland bei der Verwendung der sechs Fintierstile sowie die Signifikanzen nach der $\mathrm{X}^{2}$ - Test Methode.

Tab 20: Die amerikanischen $(n=4)$ Fintierstile unterscheiden sich von den russischen $(n=11)$ Fintierstilen.

\begin{tabular}{|c|c|c|c|}
\hline Fintierstile & USA & RUS & Summe \\
\hline EFB & 89 & 151 & 240 \\
\hline EFR & 65 & 118 & 183 \\
\hline EFA & 86 & 243 & 329 \\
\hline KFBRHSV & 49 & 141 & 190 \\
\hline KFBAHSV & 108 & 202 & 310 \\
\hline KFBAHSZ & 8 & 15 & 23 \\
\hline Summe & 405 & 870 & 1275 \\
\hline
\end{tabular}




\begin{tabular}{|l|l|c|c|c|c|c|}
\hline Fintierstiele & Länder & $\mathbf{F b}$ & $\mathbf{F e}$ & $\mathbf{( F b - F e )}$ & $\mathbf{( F b - F e})^{\mathbf{2}}$ & $\begin{array}{c}(\mathbf{F b}-\mathbf{F e})^{2} \\
\mathbf{F e}\end{array}$ \\
\hline EFB & USA & 89 & 76,2 & 12,8 & 163,8 & 2,15 \\
\hline EFB & RUS & 151 & 163,8 & $-12,8$ & 163,8 & 1 \\
\hline EFR & USA & 65 & 58,1 & 6,9 & 47,6 & 0,82 \\
\hline EFR & RUS & 118 & 124,9 & $-6,9$ & 47,6 & 0,38 \\
\hline EFA & USA & 86 & 104,5 & $-18,5$ & 342,3 & 3,27 \\
\hline EFA & RUS & 243 & 224,5 & 18,5 & 342,3 & 1,52 \\
\hline KFBRHSV & USA & 49 & 60,4 & $-11,4$ & 130 & 2,15 \\
\hline KFBRHSV & RUS & 141 & 129,6 & 11,4 & 130 & 1 \\
\hline KFBAHSV & USA & 108 & 98,5 & 9,5 & 90,25 & 0,91 \\
\hline KFBAHSV & RUS & 202 & 211,5 & $-9,5$ & 90,25 & 0,43 \\
\hline KFBAHSZ & USA & 8 & 7,3 & 0,7 & 0,49 & 0,07 \\
\hline KFBAHSZ & RUS & 15 & 15,7 & $-0,7$ & 0,49 & 0,3 \\
\hline Summe & & 1275 & 1275 & 0 & & $\mathbf{X}^{\mathbf{2}=14}$ \\
\hline
\end{tabular}

Der $X^{2}$ - Wert $=11,1$ signifikant auf dem Niveau von 0,05 (Zweiseitig)

Der $X^{2}$ - Wert $=20,5$ signifikant auf dem Niveau von 0,01 (Zweiseitig) $X^{2}=14>11,1$, d. h. das Ergebnis ist signifikant auf dem 0,05 Niveau.

In der Tab. 20 sind die Fintierstile für die Boxer aus den USA und die Fintierstile für die Boxer aus Russland nach der $\mathbf{X}^{2}$ - Test Methode dargestellt. Die russischen Boxer verwenden die Fintierstile anders als die amerikanischen Boxer. 


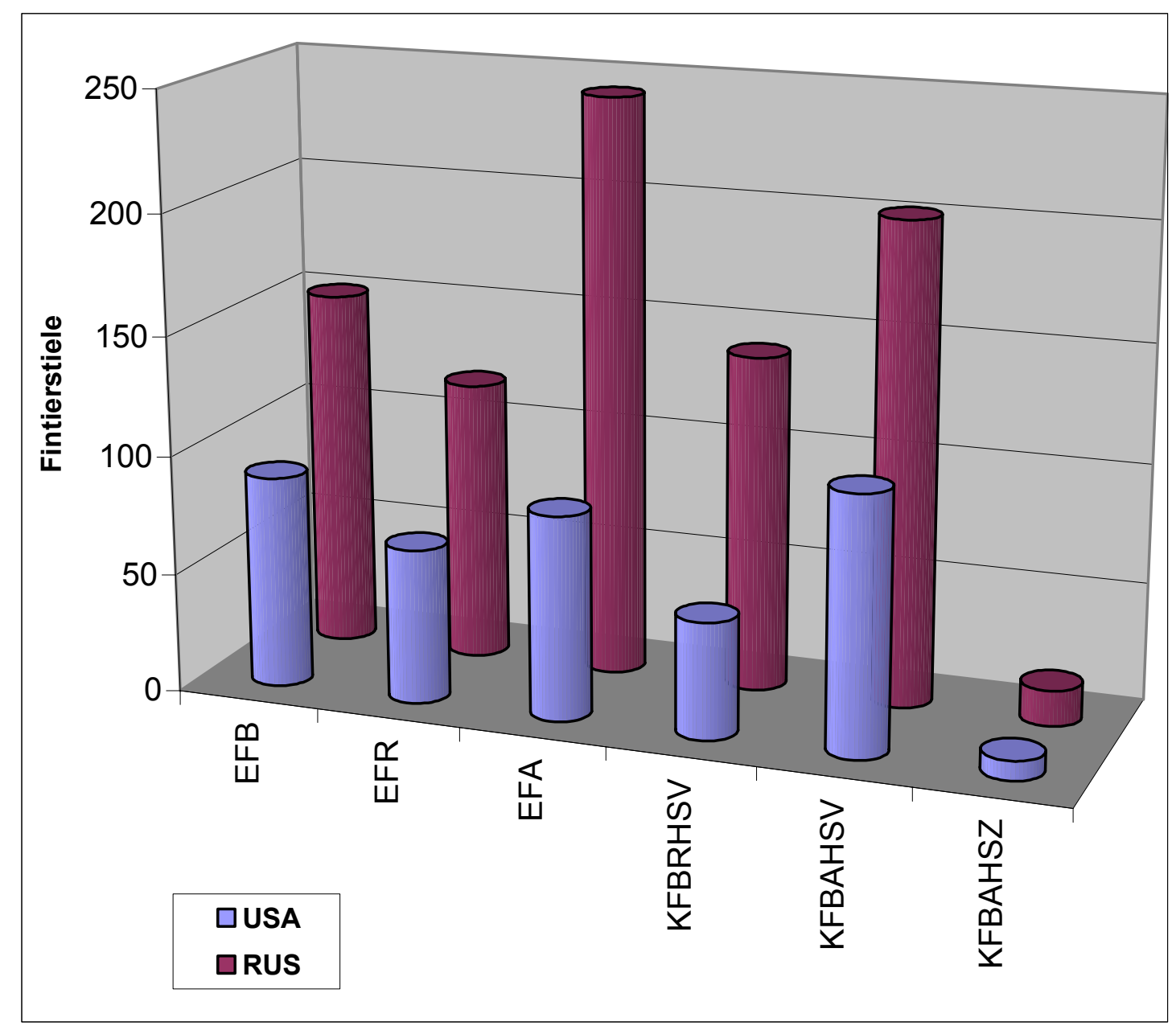

Abb. 57: Die amerikanischen $(n=4)$ Fintierstile unterscheiden sich von den russischen $(n=11)$ Fintierstilen.

In der Abb. 57 ist die Häufigkeit der Fintierstile der Boxer aus den USA und der Boxer aus Russland aufgezeigt. Die russischen Boxer verwenden die Fintierstile anders an als die amerikanischen Boxer. 
Tabelle 21 zeigt den Unterschied zwischen den Boxern aus den USA und den Boxern aus der Ukraine bei der Verwendung der sechs Fintierstile sowie die Signifikanzen nach der $\mathrm{X}^{2}$ - Test Methode.

Tab 21: Die amerikanischen $(n=4)$ Fintierstile unterscheiden sich von den ukrainischen $(n=5)$ Fintierstilen.

\begin{tabular}{|c|c|c|c|}
\hline Fintierstile & USA & UKR & Summe \\
\hline EFB & 89 & 93 & 182 \\
\hline EFR & 65 & 90 & 155 \\
\hline EFA & 86 & 55 & 141 \\
\hline KFBRHSV & 49 & 90 & 139 \\
\hline KFBAHSV & 108 & 103 & 211 \\
\hline KFBAHSZ & 8 & 3 & 11 \\
\hline Summe & 405 & 434 & 839 \\
\hline
\end{tabular}

\begin{tabular}{|c|c|c|c|c|c|c|}
\hline Fintierstiele & Länder & $\mathrm{Fb}$ & $\mathrm{Fe}$ & (Fb-Fe) & $\begin{array}{l}(\mathrm{Fb}- \\
\mathrm{Fe})^{2}\end{array}$ & $\frac{(\mathrm{Fb}-\mathrm{Fe})^{2}}{\mathrm{Fe}}$ \\
\hline EFB & USA & 89 & 87,9 & 1,1 & 1,21 & 0,01 \\
\hline EFB & UKR & 93 & 94,1 & $-1,1$ & 1,21 & 0,01 \\
\hline EFR & USA & 65 & 74,8 & $-9,8$ & 96,04 & 1,28 \\
\hline EFR & UKR & 90 & 80,2 & 9,8 & 96,04 & 1,19 \\
\hline EFA & USA & 86 & 68,1 & 17,9 & 320,41 & 4,70 \\
\hline EFA & UKR & 55 & 72,9 & $-17,9$ & 320,41 & 4,39 \\
\hline KFBRHSV & USA & 49 & 67,1 & $-18,1$ & 327,61 & 4,88 \\
\hline KFBRHSV & UKR & 90 & 71,9 & 18,1 & 327,61 & 4,56 \\
\hline KFBAHSV & USA & 108 & 101,9 & 6,1 & 37,21 & 0,37 \\
\hline KFBAHSV & UKR & 103 & 109,1 & $-6,1$ & 37,21 & 0,34 \\
\hline KFBAHSZ & USA & 8 & 5,3 & 2,7 & 7,29 & 1,37 \\
\hline KFBAHSZ & UKR & 3 & 5,7 & $-2,7$ & 7,29 & 1,28 \\
\hline Summe & & 839 & 839 & 0 & & $X^{2}=24,38$ \\
\hline
\end{tabular}

Der $X^{2}$ - Wert $=11,1$ signifikant auf dem Niveau von 0,05 (Zweiseitig) Der $X^{2}$ - Wert $=20,5$ signifikant auf dem Niveau von 0,01 (Zweiseitig) $X^{2}=24,38>20,5$, d. h. das Ergebnis ist hoch signifikant auf dem 0,01 Niveau. 
In der Tab. 21 sind die Fintierstile für die Boxer aus den USA und die Fintierstile für die Boxer aus der Ukraine nach der $\mathbf{X}^{2}$ - Test Methode dargestellt. Die ukrainischen Boxer verwenden die Fintierstile anders als die amerikanischen Boxer.

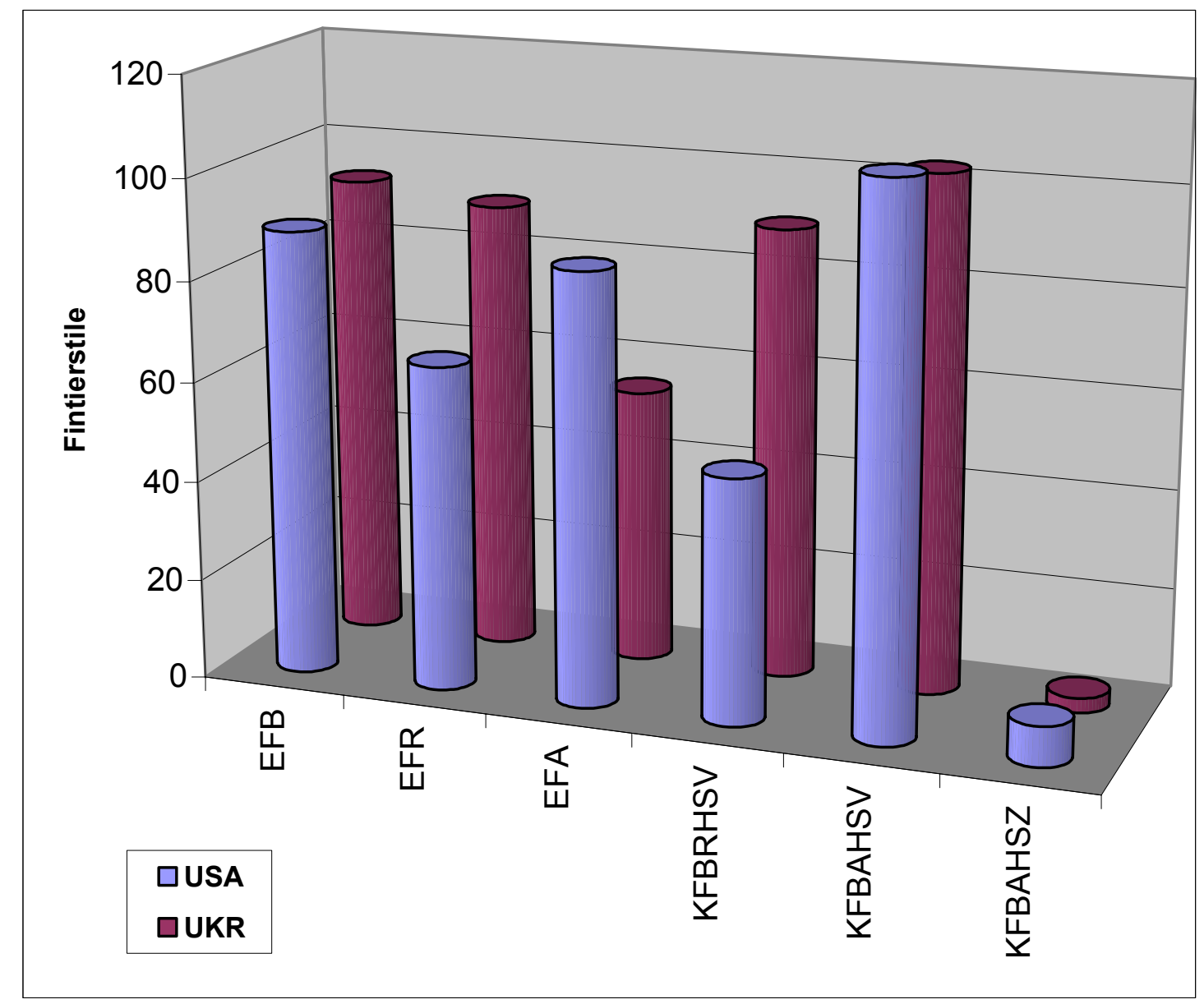

Abb. 58: Die amerikanischen $(n=4)$ Fintierstile unterscheiden sich von den ukrainischen $(n=5)$ Fintierstilen.

In der Abb. 58 ist die Häufigkeit der Fintierstile der Boxer aus den USA und der Boxer aus der Ukraine aufgezeigt. Die ukrainischen Boxer verwenden die Fintierstile anders an als die amerikanischen Boxer. 
Tabelle 22 zeigt den Unterschied zwischen den Boxern aus den USA und den Boxern aus Kuba bei der Verwendung der sechs Fintierstile sowie die Signifikanzen nach der $\mathrm{X}^{2}$ - Test Methode.

Tab 22: Die amerikanischen $(n=4)$ Fintierstile unterscheiden sich von den kubanischen $(n=8)$ Fintierstilen.

\begin{tabular}{|c|c|c|c|}
\hline Fintierstile & USA & CUB & Summe \\
\hline EFB & 89 & 267 & 356 \\
\hline EFR & 65 & 157 & 222 \\
\hline EFA & 86 & 131 & 217 \\
\hline KFBRHSV & 49 & 143 & 192 \\
\hline KFBAHSV & 108 & 317 & 425 \\
\hline KFBAHSZ & 8 & 3 & 11 \\
\hline Summe & 405 & 1018 & 1423 \\
\hline
\end{tabular}

\begin{tabular}{|c|c|c|c|c|c|c|}
\hline Fintierstiele & Länder & $\mathbf{F b}$ & $\mathrm{Fe}$ & (Fb-Fe) & $\begin{array}{l}(\mathrm{Fb}- \\
\mathrm{Fe})^{2}\end{array}$ & $\frac{(\mathrm{Fb}-\mathrm{Fe})^{2}}{\mathrm{Fe}}$ \\
\hline EFB & USA & 89 & 101,3 & $-12,3$ & 151,29 & 1,49 \\
\hline EFB & CUB & 267 & 254,7 & 12,3 & 151,29 & 0,59 \\
\hline EFR & USA & 65 & 63,2 & 1,8 & 3,24 & 0,05 \\
\hline EFR & CUB & 157 & 158,8 & $-1,8$ & 3,24 & 0,02 \\
\hline EFA & USA & 86 & 61,8 & 24,2 & 585,64 & 9,48 \\
\hline EFA & CUB & 131 & 155,2 & $-24,2$ & 585,64 & 3,77 \\
\hline KFBRHSV & USA & 49 & 54,6 & $-5,6$ & 31,36 & 0,57 \\
\hline KFBRHSV & CUB & 143 & 137,4 & 5,6 & 31,36 & 0,23 \\
\hline KFBAHSV & USA & 108 & 121 & -13 & 169 & 1,39 \\
\hline KFBAHSV & CUB & 317 & 304 & 13 & 169 & 0,56 \\
\hline KFBAHSZ & USA & 8 & 3,1 & 4,9 & 24,01 & 7,75 \\
\hline KFBAHSZ & CUB & 3 & 7,9 & $-4,9$ & 24,02 & 3,04 \\
\hline Summe & & 1423 & 1423 & 0 & & $X^{2}=28,94$ \\
\hline
\end{tabular}

Der $X^{2}$ - Wert $=11,1$ signifikant auf dem Niveau von 0,05 (Zweiseitig) Der $X^{2}$ - Wert $=20,5$ signifikant auf dem Niveau von 0,01 (Zweiseitig) $X^{2}=28,94>20,5, d$. h. das Ergebnis ist hoch signifikant auf dem 0,01 Niveau.

In der Tab. 22 sind die Fintierstile für die Boxer aus den USA und die Fintierstile für die Boxer aus Kuba nach der $\mathbf{X}^{2}$ - Test Methode dargestellt. Die kubanischen Boxer verwenden die Fintierstile anders als die amerikanischen Boxer. 


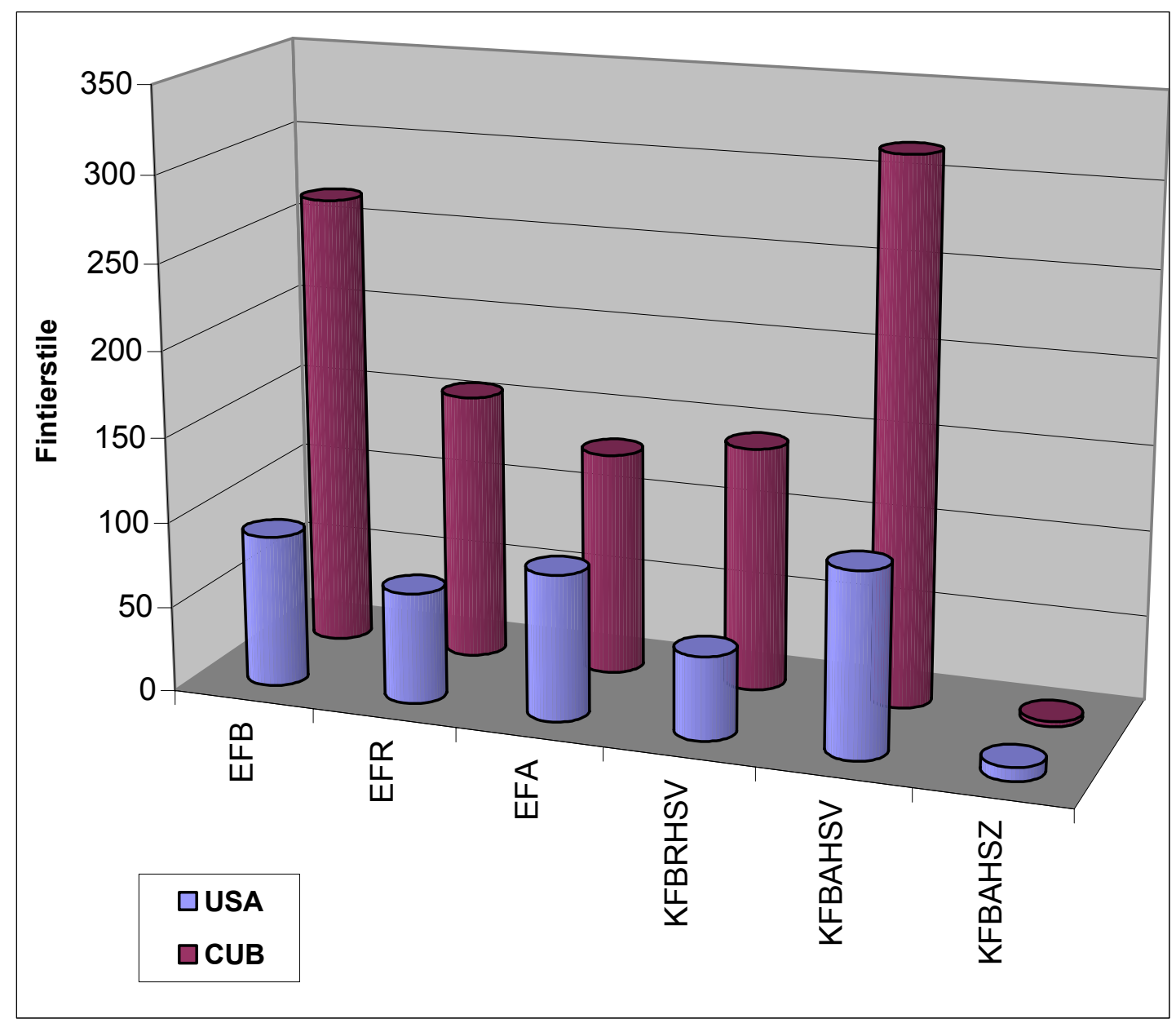

Abb. 59: Die amerikanischen $(n=4)$ Fintierstile unterscheiden sich von den kubanischen $(n=8)$ Fintierstilen.

In der Abb. 59 ist die Häufigkeit der Fintierstile der Boxer aus den USA und der Boxer aus Kuba aufgezeigt. Die kubanischen Boxer verwenden die Fintierstile anders an als die amerikanischen Boxer. 
Tabelle 23 zeigt den Unterschied zwischen den Boxern aus den USA und den Boxern aus Kasachstan bei der Verwendung der sechs Fintierstile sowie die Signifikanzen nach der $\mathrm{X}^{2}$ - Test Methode.

Tab 23: Die amerikanischen $(n=4)$ Fintierstile unterscheiden sich von den kasachischen $(n=6)$ Fintierstilen.

\begin{tabular}{|l|c|c|c|}
\hline Fintierstile & USA & KAZ & Summe \\
\hline EFB & 89 & 132 & 221 \\
\hline EFR & 65 & 108 & 173 \\
\hline EFA & 86 & 119 & 205 \\
\hline KFBRHSV & 49 & 126 & 175 \\
\hline KFBAHSV & 108 & 167 & 275 \\
\hline KFBAHSZ & 8 & 5 & 13 \\
\hline \multicolumn{1}{|c|}{ Summe } & 405 & 657 & 1062 \\
\hline
\end{tabular}

\begin{tabular}{|c|c|c|c|c|c|c|}
\hline Fintierstiele & Länder & $\mathbf{F b}$ & $\mathrm{Fe}$ & (Fb-Fe) & $(\mathrm{Fb}-\mathrm{Fe})^{2}$ & $\frac{(\mathrm{Fb}-\mathrm{Fe})^{2}}{\mathrm{Fe}}$ \\
\hline EFB & USA & 89 & 84,3 & 4,7 & 22,09 & 0,62 \\
\hline EFB & KAZ & 132 & 136,7 & $-4,7$ & 22,09 & 0,16 \\
\hline EFR & USA & 65 & 66 & -1 & 1 & 0,02 \\
\hline EFR & KAZ & 108 & 107 & 1 & 1 & 0 \\
\hline EFA & USA & 86 & 78,2 & 7,8 & 60,84 & 0,78 \\
\hline EFA & KAZ & 119 & 126,8 & $-7,8$ & 60,84 & 0,48 \\
\hline KFBRHSV & USA & 49 & 66,7 & $-17,7$ & 313,29 & 4,69 \\
\hline KFBRHSV & KAZ & 126 & 108,3 & 17,7 & 313,29 & 2,89 \\
\hline KFBAHSV & USA & 108 & 104,9 & 3,1 & 9,61 & 0,09 \\
\hline KFBAHSV & KAZ & 167 & 170,1 & $-3,1$ & 9,61 & 0,05 \\
\hline KFBAHSZ & USA & 8 & 5 & 3 & 9 & 1,8 \\
\hline KFBAHSZ & KAZ & 5 & 8 & -3 & 9 & 1,12 \\
\hline Summe & & 1062 & 1062 & 0 & & $X^{2}=12,7$ \\
\hline
\end{tabular}

Der $X^{2}$ - Wert $=11,1$ signifikant auf dem Niveau von 0,05 (Zweiseitig) Der $X^{2}$ - Wert $=20,5$ signifikant auf dem Niveau von 0,01 (Zweiseitig) $X^{2}=12,7>11,1$, d. h. das Ergebnis ist signifikant auf dem 0,05 Niveau. 
In der Tab. 23 sind die Fintierstile für die Boxer aus den USA und die Fintierstile für die Boxer aus Kasachstan nach der $\mathbf{X}^{\mathbf{2}}$ - Test Methode dargestellt. Die kasachischen Boxer verwenden die Fintierstile anders als die amerikanischen Boxer.

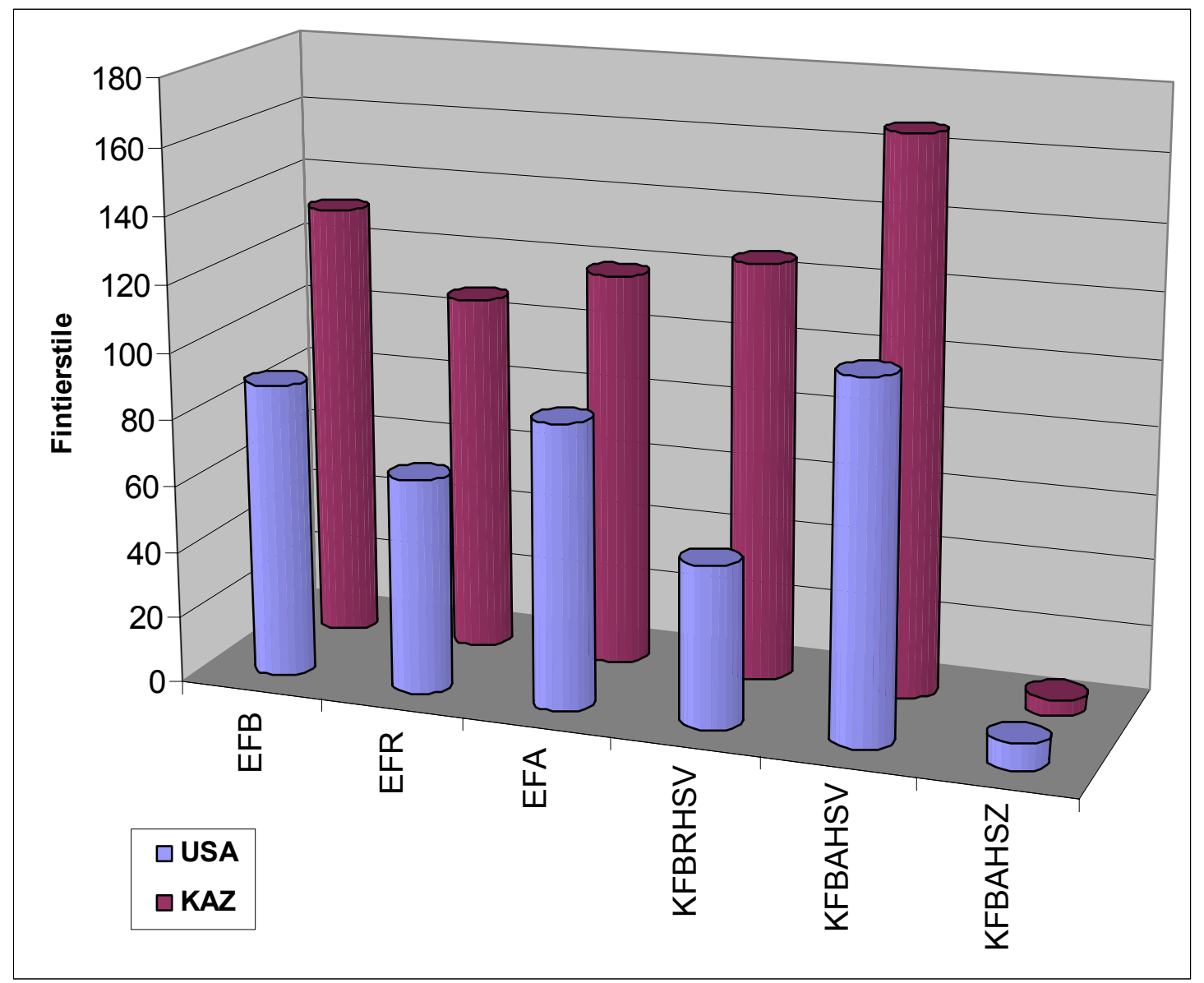

Abb. 60: Die amerikanischen $(n=4)$ Fintierstile unterscheiden sich von den kasachischen $(n=6)$ Fintierstilen.

In der Abb. 60 ist die Häufigkeit der Fintierstile der Boxer aus den USA und der Boxer aus Kasachstan aufgezeigt. Die kasachischen Boxer verwenden die Fintierstile anders an als die amerikanischen Boxer. 


\subsubsection{Die russische Boxschule unterscheidet sich von denen aus der Ukraine, Kuba und Kasachstan}

Tabelle 24 zeigt den Unterschied zwischen den Boxern aus Russland und den Boxern aus der Ukraine bei der Verwendung der sechs Fintierstile sowie die Signifikanzen nach dem $\mathrm{X}^{2}$ - Test.

Tab 24: Die russischen $(n=11)$ Fintierstile unterscheiden sich von den ukrainischen $(n=5)$ Fintierstilen.

\begin{tabular}{|c|c|c|c|}
\hline Fintierstile & RUS & UKR & Summe \\
\hline EFB & 151 & 93 & 244 \\
\hline EFR & 118 & 90 & 208 \\
\hline EFA & 243 & 55 & 298 \\
\hline KFBRHSV & 141 & 90 & 231 \\
\hline KFBAHSV & 202 & 103 & 305 \\
\hline KFBAHSZ & 15 & 3 & 18 \\
\hline Summe & 870 & 434 & 1304 \\
\hline
\end{tabular}

\begin{tabular}{|l|c|c|c|c|c|c|}
\hline Fintierstiele & Länder & $\mathbf{F b}$ & $\mathbf{F e}$ & $\mathbf{( F b - F e )}$ & $\mathbf{( F b - F e})^{\mathbf{2}}$ & $\begin{array}{c}(\mathbf{F b}-\mathbf{F e})^{2} \\
\mathbf{F e}\end{array}$ \\
\hline EFB & RUS & 151 & 162,8 & $-11,8$ & 139,24 & 0,86 \\
\hline EFB & UKR & 93 & 81,2 & 11,8 & 139,24 & 1,71 \\
\hline EFR & RUS & 118 & 138,8 & $-20,8$ & 434,64 & 3,13 \\
\hline EFR & UKR & 90 & 69,2 & 20,8 & 434,64 & 6,28 \\
\hline EFA & RUS & 243 & 198,8 & 44,2 & 1953,64 & 9,83 \\
\hline EFA & UKR & 55 & 99,2 & $-44,2$ & 1953,64 & 19,69 \\
\hline KFBRHSV & RUS & 141 & 154,1 & $-13,1$ & 171,61 & 1,11 \\
\hline KFBRHSV & UKR & 90 & 76,9 & 13,1 & 171,61 & 2,23 \\
\hline KFBAHSV & RUS & 202 & 203,5 & $-1,5$ & 2,25 & 0,01 \\
\hline KFBAHSV & UKR & 103 & 101,5 & 1,5 & 2,25 & 0,02 \\
\hline KFBAHSZ & RUS & 15 & 12 & 3 & 9 & 0,75 \\
\hline KFBAHSZ & UKR & 3 & 6 & -3 & 9 & 1,5 \\
\hline Summe & & 1304 & 1304 & 0 & & $\mathbf{X}^{\mathbf{2}=\mathbf{4 7 , 1 2}}$ \\
\hline
\end{tabular}

Der $X^{2}$ - Wert $=11,1$ signifikant auf dem Niveau von 0,05 (Zweiseitig) Der $X^{2}$ - Wert $=20,5$ signifikant auf dem Niveau von 0,01 (Zweiseitig) $X^{2}=47,12>20,5$, d. h. das Ergebnis ist hoch signifikant auf dem 0,01 Niveau. 
In der Tab. 24 sind die Fintierstile für die Boxer aus Russland und die Fintierstile für die Boxer aus der Ukraine nach der $\mathbf{X}^{2}$ - Test Methode dargestellt. Die russischen Boxer verwenden die Fintierstile anders als die ukrainischen Boxer.

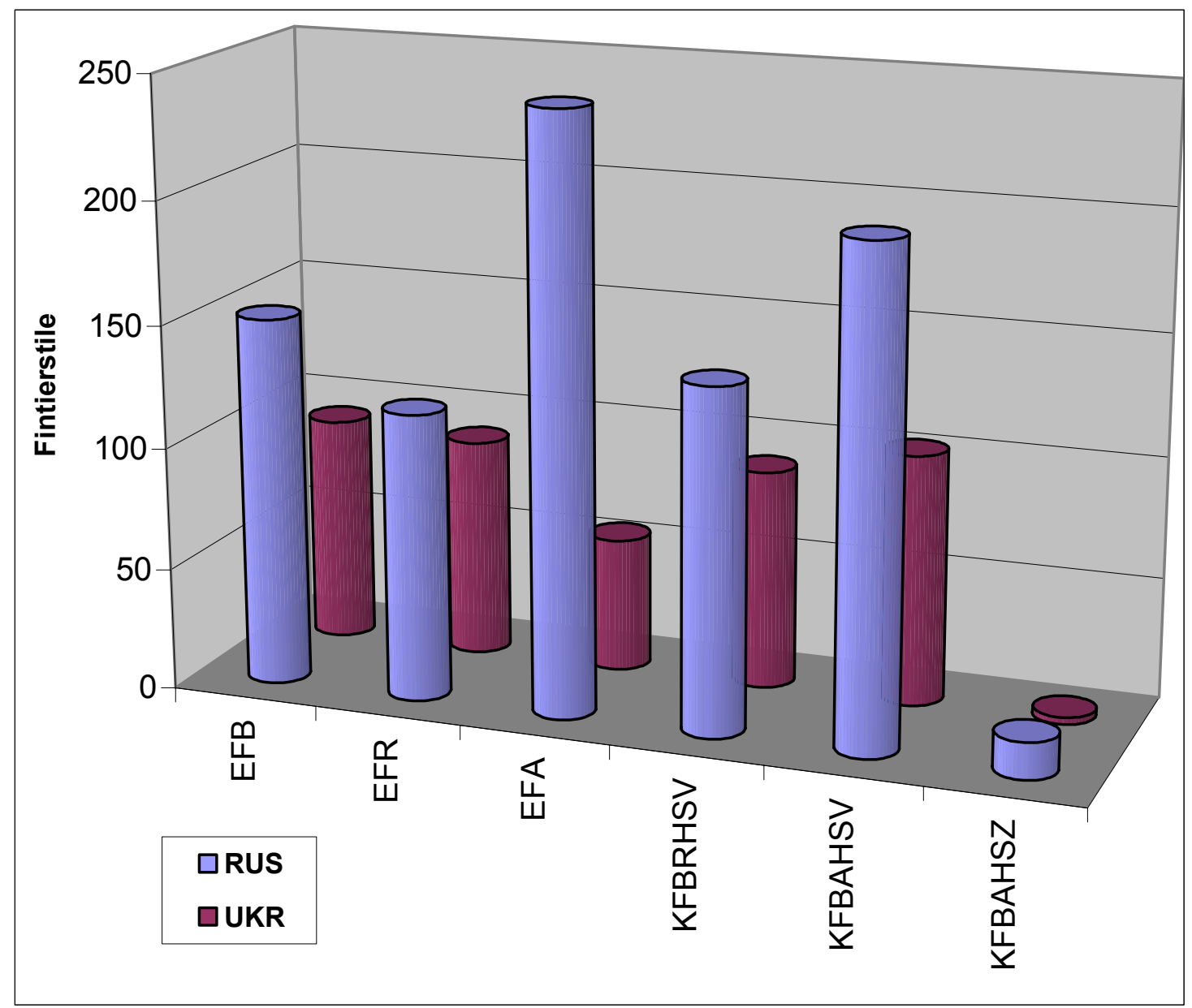

\section{Abb. 61: Die russischen $(n=11)$ Fintierstile unterscheiden sich von den uk- rainischen $(n=5)$ Fintierstilen.}

In der Abb. 61 ist die Häufigkeit der Fintierstile der Boxer aus Russland und der Boxer aus der Ukraine aufgezeigt. Die russischen Boxer verwenden die Fintierstile anders an als die ukrainischen Boxer. 
Tabelle 25 zeigt den Unterschied zwischen den Boxern aus Russland und den Boxern aus Kuba bei der Verwendung der sechs Fintierstile sowie die Signifikanzen nach dem $\mathrm{X}^{2}$ - Test.

Tab 25: Die russischen $(n=11)$ Fintierstile unterscheiden sich von den kubanischen $(n=8)$ Fintierstilen.

\begin{tabular}{|c|c|c|c|}
\hline Fintierstile & RUS & CUB & Summe \\
\hline EFB & 151 & 267 & 418 \\
\hline EFR & 118 & 157 & 275 \\
\hline EFA & 243 & 131 & 374 \\
\hline KFBRHSV & 141 & 143 & 284 \\
\hline KFBAHSV & 202 & 317 & 519 \\
\hline KFBAHSZ & 15 & 3 & 18 \\
\hline Summe & 870 & 1018 & 1888 \\
\hline
\end{tabular}

\begin{tabular}{|c|c|c|c|c|c|c|}
\hline Fintierstiele & Länder & $\mathrm{Fb}$ & $\mathrm{Fe}$ & (Fb-Fe) & $(\mathrm{Fb}-\mathrm{Fe})^{2}$ & $\frac{(\mathrm{Fb}-\mathrm{Fe})^{2}}{\mathrm{Fe}}$ \\
\hline EFB & RUS & 151 & 192,6 & $-41,6$ & 1730,56 & 8,98 \\
\hline EFB & CUB & 267 & 225,4 & 41,6 & 1730,56 & 7,67 \\
\hline EFR & RUS & 118 & 126,7 & $-8,7$ & 75,67 & 0,59 \\
\hline EFR & CUB & 157 & 148,3 & 8,7 & 75,67 & 0,51 \\
\hline EFA & RUS & 243 & 172,3 & 70,7 & 4998,49 & 29,01 \\
\hline EFA & CUB & 131 & 201,7 & $-70,7$ & 4998,49 & 24,78 \\
\hline KFBRHSV & RUS & 141 & 130,9 & 10,1 & 102,01 & 0,77 \\
\hline KFBRHSV & CUB & 143 & 153,1 & $-10,1$ & 102,01 & 0,66 \\
\hline KFBAHSV & RUS & 202 & 239,2 & $-37,2$ & 1383,84 & 5,78 \\
\hline KFBAHSV & CUB & 317 & 279,8 & 37,2 & 1383,84 & 4,94 \\
\hline KFBAHSZ & RUS & 15 & 8,3 & 6,7 & 44,89 & 5,41 \\
\hline KFBAHSZ & CUB & 3 & 9,7 & $-6,7$ & 44,89 & 4,63 \\
\hline Summe & & 1888 & 1888 & 0 & & $x^{2}=93,73$ \\
\hline
\end{tabular}

Der $X^{2}$ - Wert $=11,1$ signifikant auf dem Niveau von 0,05 (Zweiseitig) Der $X^{2}$ - Wert $=20,5$ signifikant auf dem Niveau von 0,01 (Zweiseitig) $X^{2}=93,73>20,5$, d. h. das Ergebnis ist hoch signifikant auf dem 0,01 Niveau. 
In der Tab. 25 sind die Fintierstile für die Boxer aus Russland und die Fintierstile für die Boxer aus Kuba nach der $\mathbf{X}^{\mathbf{2}}$ - Test Methode dargestellt. Die kubanischen Boxer verwenden die Fintierstile anders als die russischen Boxer.

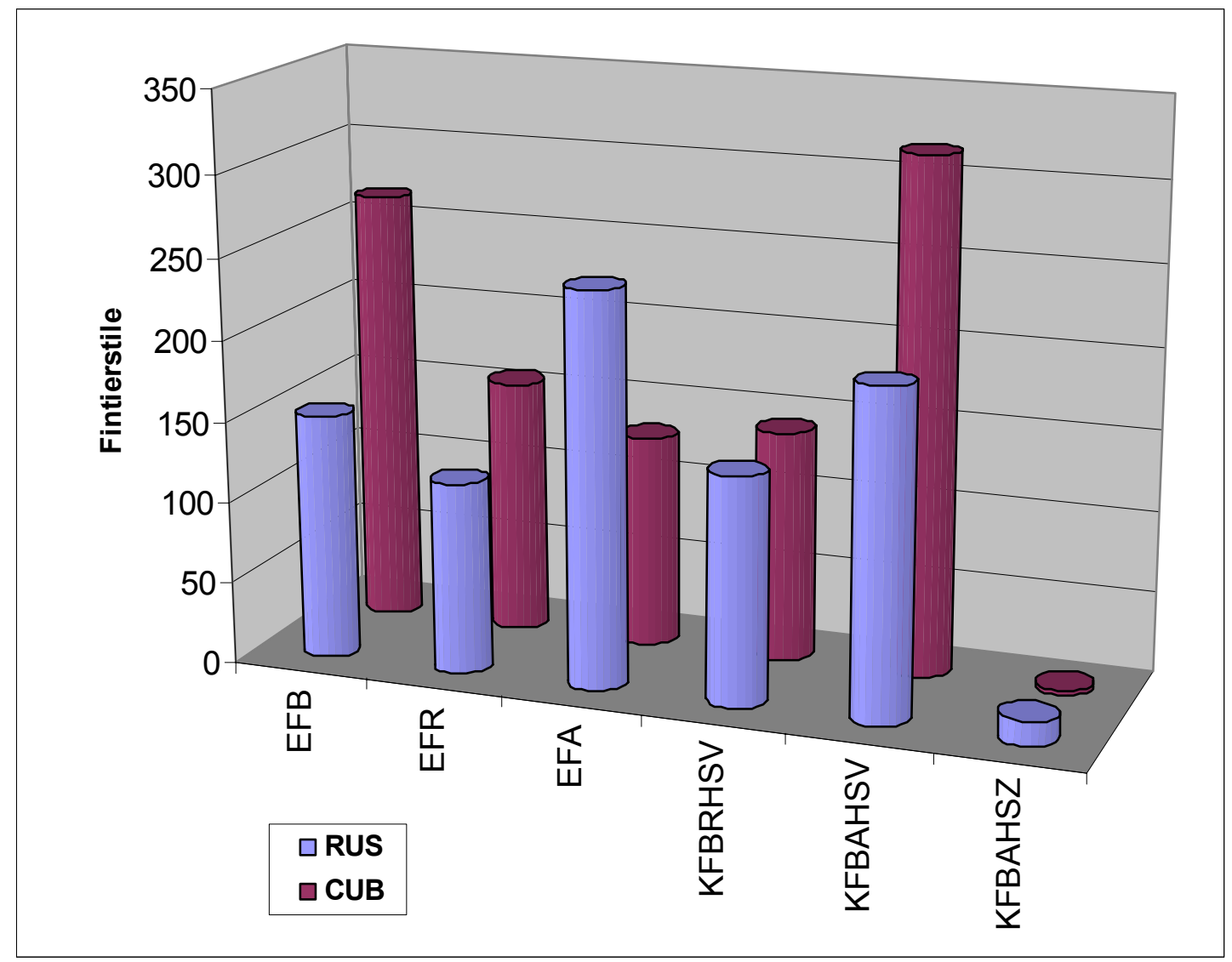

Abb. 62: Die russischen $(n=11)$ Fintierstile unterscheiden sich von den kubanischen $(n=8)$ Fintierstilen.

In der Abb. 62 ist die Häufigkeit der Fintierstile der Boxer aus Russland und der Boxer aus Kuba aufgezeigt. Die kubanischen Boxer verwenden die Fintierstile anders an als die russischen Boxer. 
Tabelle 26 zeigt den Unterschied zwischen den Boxern aus Russland und den Boxern aus Kasachstan bei der Verwendung der sechs Fintierstile sowie die Signifikanzen nach dem $\mathrm{X}^{2}$ - Test.

Tab 26: Die russischen $(n=11)$ Fintierstile unterscheiden sich von den kasachischen $(n=6)$ Fintierstilen.

\begin{tabular}{|c|c|c|c|}
\hline Fintierstile & RUS & KAZ & Summe \\
\hline EFB & 151 & 132 & 283 \\
\hline EFR & 118 & 108 & 226 \\
\hline EFA & 243 & 119 & 362 \\
\hline KFBRHSV & 141 & 126 & 267 \\
\hline KFBAHSV & 202 & 167 & 369 \\
\hline KFBAHSZ & 15 & 5 & 20 \\
\hline Summe & 870 & 657 & 1527 \\
\hline
\end{tabular}

\begin{tabular}{|l|c|c|c|c|c|c|}
\hline Fintierstiele & Länder & $\mathbf{F b}$ & $\mathbf{F e}$ & $\mathbf{( F b - F e )}$ & $\mathbf{( F b - F e ) ~}^{\mathbf{2}}$ & $\begin{array}{c}(\mathbf{F b}-\mathbf{F e})^{2} \\
\mathbf{F e}\end{array}$ \\
\hline EFB & RUS & 151 & 161,2 & $-10,2$ & 104,04 & 0,65 \\
\hline EFB & KAZ & 132 & 121,8 & 10,2 & 104,04 & 0,85 \\
\hline EFR & RUS & 118 & 128,8 & $-10,8$ & 116,64 & 0,91 \\
\hline EFR & KAZ & 108 & 97,2 & 10,8 & 116,64 & 1,2 \\
\hline EFA & RUS & 243 & 206,2 & 36,8 & 1354,24 & 6,57 \\
\hline EFA & KAZ & 119 & 155,8 & $-36,8$ & 1354,24 & 8,69 \\
\hline KFBRHSV & RUS & 141 & 152,1 & $-11,1$ & 123,21 & 0,81 \\
\hline KFBRHSV & KAZ & 126 & 114,9 & 11,1 & 123,21 & 1,07 \\
\hline KFBAHSV & RUS & 202 & 210,2 & $-8,2$ & 67,24 & 0,32 \\
\hline KFBAHSV & KAZ & 167 & 158,8 & 8,2 & 67,24 & 0,42 \\
\hline KFBAHSZ & RUS & 15 & 11,4 & 3,6 & 12,96 & 1,14 \\
\hline KFBAHSZ & KAZ & 5 & 8,6 & $-3,6$ & 12,96 & 1,51 \\
\hline Summe & & 1527 & 1527 & 0 & & $\mathbf{X}^{\mathbf{2}=\mathbf{2 4 , 1 4}}$ \\
\hline
\end{tabular}

Der $X^{2}$ - Wert $=11,1$ signifikant auf dem Niveau von 0,05 (Zweiseitig) Der $X^{2}$ - Wert $=20,5$ signifikant auf dem Niveau von 0,01 (Zweiseitig) $X^{2}=24,14>20,5$, d. h. das Ergebnis ist hoch signifikant auf dem 0,01 Niveau. 
In der Tab. 26 sind die Fintierstile für die Boxer aus Russland und die Fintierstile für die Boxer aus Kasachstan nach der $\mathbf{X}^{2}$ - Test Methode dargestellt. Die russischen Boxer verwenden die Fintierstile anders als die kasachischen Boxer.

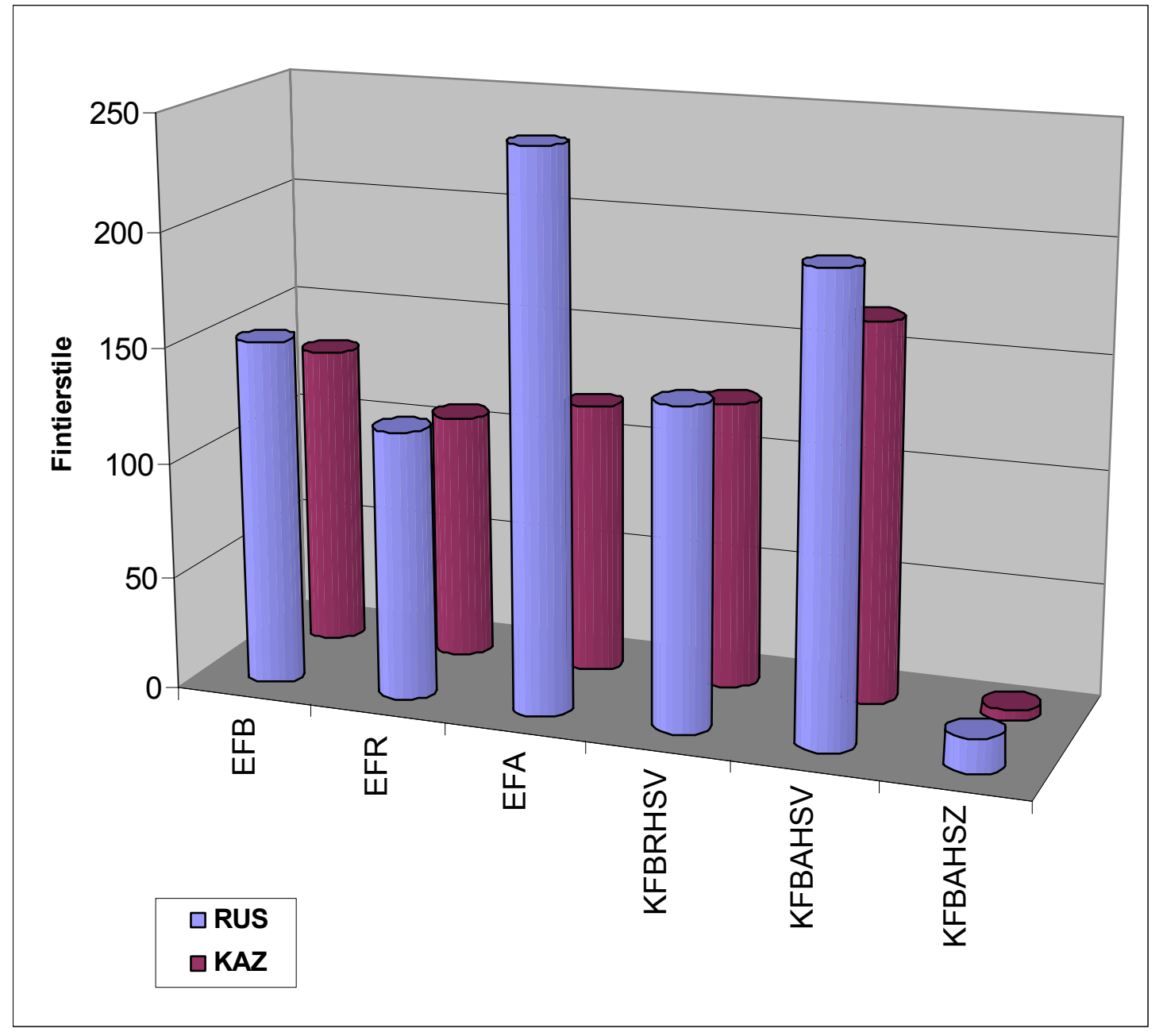

Abb. 63: Die russischen ( $n=11)$ Fintierstile unterscheiden sich von den kasachischen $(n=6)$ Fintierstilen.

In der Abb. 63 ist die Häufigkeit der Fintierstile der Boxer aus Russland und der Boxer aus Kasachstan aufgezeigt. Die russischen Boxer verwenden die Fintierstile anders an als die kasachischen Boxer. 


\subsubsection{Die kubanische Boxschule unterscheidet sich von denen aus Ukraine und Kasachstan}

Tabelle 27 zeigt den Unterschied zwischen den Boxern aus Kuba und den Boxern aus der Ukraine bei der Verwendung der sechs Fintierstile sowie die Signifikanzen nach dem $\mathrm{X}^{2}$-Test.

Tab 27: Die kubanischen $(n=8)$ Fintierstile unterscheiden sich von den ukrainischen $(n=5)$ Fintierstilen.

\begin{tabular}{|c|c|c|c|}
\hline Fintierstile & CUB & UKR & Summe \\
\hline EFB & 267 & 93 & 360 \\
\hline EFR & 157 & 90 & 247 \\
\hline EFA & 131 & 55 & 186 \\
\hline KFBRHSV & 143 & 90 & 233 \\
\hline KFBAHSV & 317 & 103 & 420 \\
\hline KFBAHSZ & 3 & 3 & 6 \\
\hline Summe & 1018 & 434 & 1452 \\
\hline
\end{tabular}

\begin{tabular}{|c|c|c|c|c|c|c|}
\hline Fintierstiele & Länder & $\mathbf{F b}$ & $\mathrm{Fe}$ & (Fb-Fe) & $\begin{array}{l}(\mathrm{Fb}- \\
\mathrm{Fe})^{2}\end{array}$ & $\frac{(\mathrm{Fb}-\mathrm{Fe})^{2}}{\mathrm{Fe}}$ \\
\hline EFB & CUB & 267 & 252,4 & 14,6 & 213,16 & 0,84 \\
\hline EFB & UKR & 93 & 107,6 & $-14,6$ & 213,16 & 1,98 \\
\hline EFR & CUB & 157 & 173,2 & $-16,2$ & 262,44 & 1,51 \\
\hline EFR & UKR & 90 & 73,8 & 16,2 & 262,44 & 3,55 \\
\hline EFA & CUB & 131 & 130,4 & 0,6 & 0,36 & 0 \\
\hline EFA & UKR & 55 & 55,6 & $-0,6$ & 0,36 & 0 \\
\hline KFBRHSV & CUB & 143 & 163,4 & $-20,4$ & 416,16 & 2,55 \\
\hline KFBRHSV & UKR & 90 & 69,6 & 20,4 & 416,16 & 5,98 \\
\hline KFBAHSV & CUB & 317 & 294,5 & 22,5 & 506,25 & 1,72 \\
\hline KFBAHSV & UKR & 103 & 125,5 & $-22,5$ & 506,25 & 4,03 \\
\hline KFBAHSZ & CUB & 3 & 4,2 & $-1,2$ & 1,44 & 0,34 \\
\hline KFBAHSZ & UKR & 3 & 1,8 & 1,2 & 1,44 & 0,8 \\
\hline Summe & & 1452 & 1452 & 0 & & $X^{2}=23,3$ \\
\hline
\end{tabular}

Der $X^{2}$ - Wert $=11,1$ signifikant auf dem Niveau von 0,05 (Zweiseitig) Der $X^{2}$ - Wert $=20,5$ signifikant auf dem Niveau von 0,01 (Zweiseitig) $X^{2}=23,3>20,5$, d. h. das Ergebnis ist hoch signifikant auf dem 0,01 Niveau. 
In der Tab. 27 sind die Fintierstile für die Boxer aus Kuba und die Fintierstile für die Boxer aus der Ukraine nach der $\mathbf{X}^{\mathbf{2}}$ - Test Methode dargestellt. Die kubanischen Boxer verwenden die Fintierstile anders als die ukrainischen Boxer.

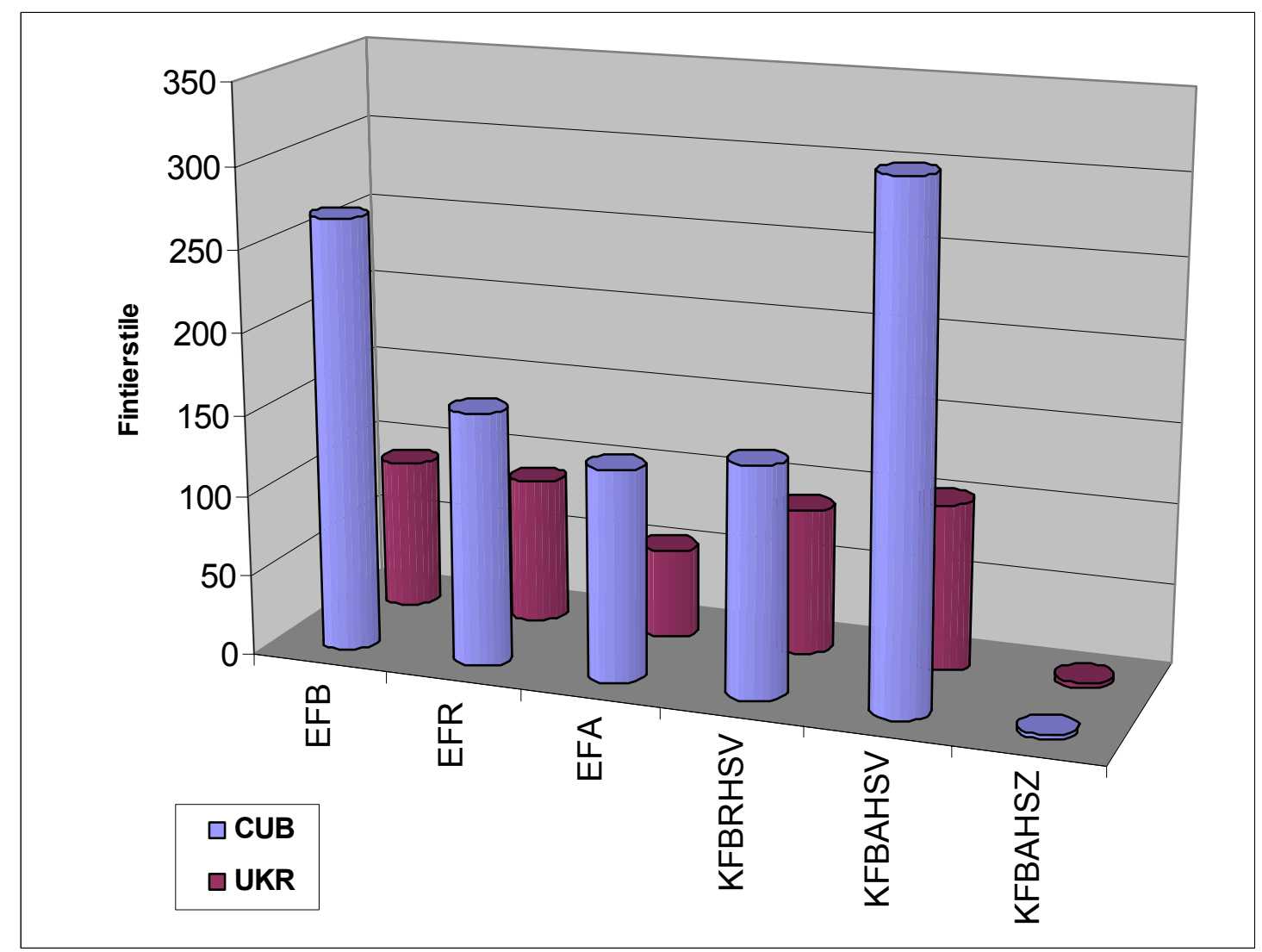

Abb. 64: Die kubanischen $(n=8)$ Fintierstile unterscheiden sich von den ukrainischen $(n=5)$ Fintierstilen.

In der Abb. 64 ist die Häufigkeit der Fintierstile der Boxer aus Kuba und der Boxer aus der Ukraine aufgezeigt. Die kubanischen Boxer verwenden die Fintierstile anders an als die ukrainischen Boxer. 
Tabelle 28 zeigt den Unterschied zwischen den Boxern aus Kuba und den Boxern aus Kasachstan bei der Verwendung der sechs Fintierstile sowie die Signifikanzen nach dem $\mathrm{X}^{2}$-Test.

Tab 28: Die kubanischen $(n=8)$ Fintierstile unterscheiden sich von den kasachischen $(n=6)$ Fintierstilen.

\begin{tabular}{|c|c|c|c|}
\hline Fintierstile & CUB & KAZ & Summe \\
\hline EFB & 267 & 132 & 399 \\
\hline EFR & 157 & 108 & 265 \\
\hline EFA & 131 & 119 & 250 \\
\hline KFBRHSV & 143 & 126 & 269 \\
\hline KFBAHSV & 317 & 167 & 484 \\
\hline KFBAHSZ & 3 & 5 & 8 \\
\hline Summe & 1018 & 657 & 1675 \\
\hline
\end{tabular}

\begin{tabular}{|c|c|c|c|c|c|c|}
\hline Fintierstiele & Länder & $\mathbf{F b}$ & $\mathrm{Fe}$ & (Fb-Fe) & $\begin{array}{l}(\mathrm{Fb}- \\
\mathrm{Fe})^{2}\end{array}$ & $\frac{(\mathrm{Fb}-\mathrm{Fe})^{2}}{\mathrm{Fe}}$ \\
\hline EFB & CUB & 267 & 242,5 & 24,5 & 600,25 & 2,47 \\
\hline EFB & KAZ & 132 & 156,5 & $-24,5$ & 600,25 & 3,83 \\
\hline EFR & CUB & 157 & 161,1 & $-4,1$ & 16,81 & 0,10 \\
\hline EFR & KAZ & 108 & 103,9 & 4,1 & 16,81 & 0,16 \\
\hline EFA & CUB & 131 & 151,9 & $-20,9$ & 436,81 & 2,87 \\
\hline EFA & KAZ & 119 & 98,1 & 20,9 & 436,81 & 4,45 \\
\hline KFBRHSV & CUB & 143 & 163,5 & $-20,5$ & 420,25 & 2,57 \\
\hline KFBRHSV & KAZ & 126 & 105,5 & 20,5 & 420,25 & 3,98 \\
\hline KFBAHSV & CUB & 317 & 294,2 & 22,8 & 519,84 & 1,76 \\
\hline KFBAHSV & KAZ & 167 & 189,8 & $-22,8$ & 519,84 & 2,73 \\
\hline KFBAHSZ & CUB & 3 & 4,9 & $-1,9$ & 3,61 & 0,73 \\
\hline KFBAHSZ & KAZ & 5 & 3,1 & 1,9 & 3,61 & 1,16 \\
\hline Summe & & 1675 & 1675 & 0 & & $X^{2}=26,81$ \\
\hline
\end{tabular}

Der $X^{2}$ - Wert $=11,1$ signifikant auf dem Niveau von 0,05 (Zweiseitig) Der $X^{2}$ - Wert $=20,5$ signifikant auf dem Niveau von 0,01 (Zweiseitig) $X^{2}=26,81>20,5$, d. h. das Ergebnis ist hoch signifikant auf dem 0,01 Niveau. 
In der Tab. 28 sind die Fintierstile für die Boxer aus Kuba und die Fintierstile für die Boxer aus Kasachstan nach der $\mathbf{X}^{\mathbf{2}}$ - Test Methode dargestellt. Die kubanischen Boxer verwenden die Fintierstile anders als die kasachischen Boxer.

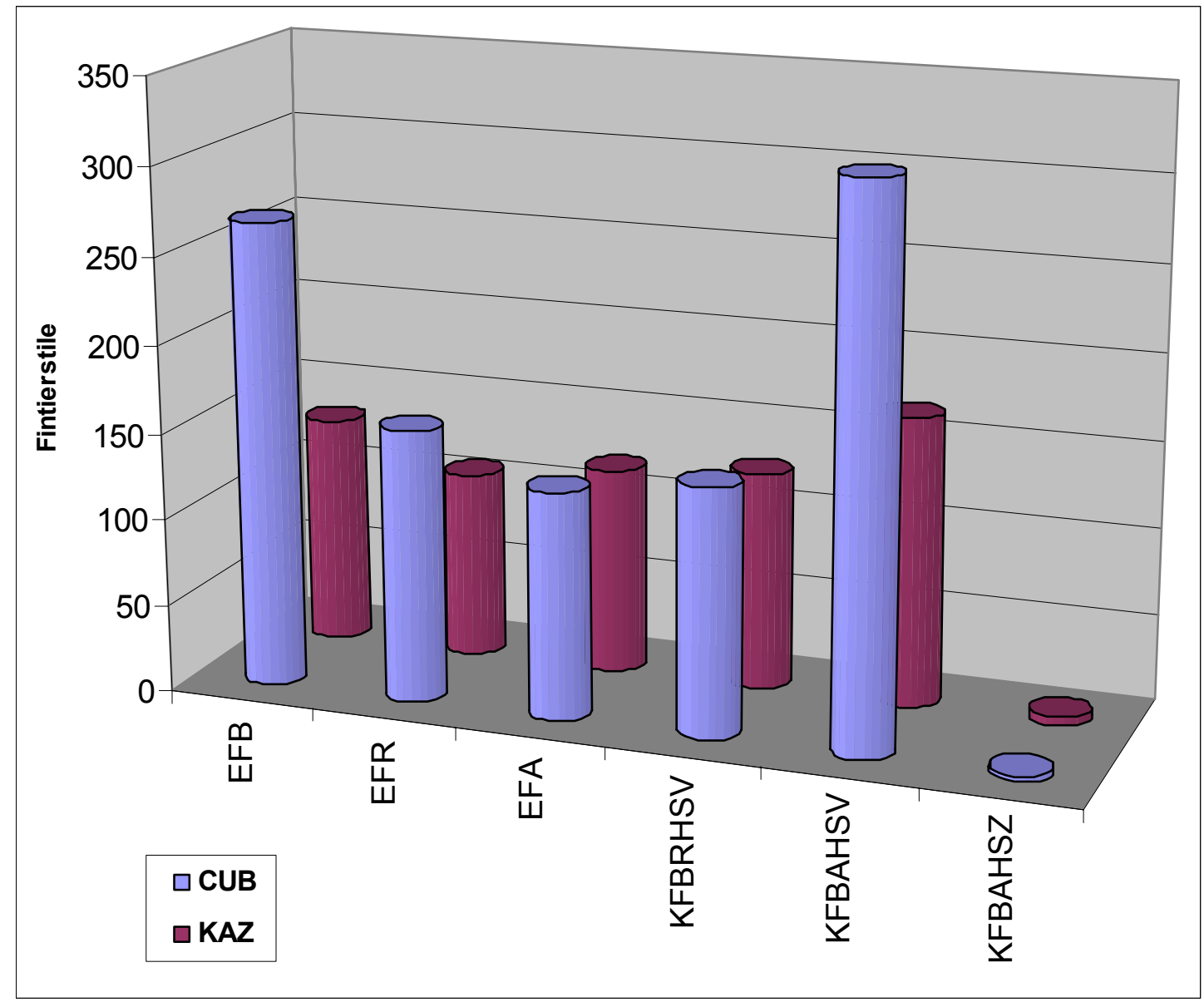

Abb. 65: Die kubanischen $(n=8)$ Fintierstile unterscheiden sich von den kasachischen $(n=6)$ Fintierstilen.

In der Abb. 65 ist die Häufigkeit der Fintierstile der Boxer aus Kuba und der Boxer aus Kasachstan aufgezeigt. Die kubanischen Boxer verwenden die Fintierstile anders an als die kasachischen Boxer. 


\subsubsection{Die kasachische Boxschule unterscheidet sich von de- nen aus der Ukraine}

Tabelle 29 zeigt den Unterschied zwischen den Boxern aus Kasachstan und den Boxern aus der Ukraine bei der Verwendung der sechs Fintierstile sowie die Signifikanzen nach dem $X^{2}$-Test.

Tab 29: Die kasachischen $(n=6)$ Fintierstile unterscheiden sich von den ukrainischen $(n=5)$ Fintierstilen.

\begin{tabular}{|c|c|c|c|}
\hline Fintierstile & KAZ & UKR & Summe \\
\hline EFB & 132 & 93 & 225 \\
\hline EFR & 108 & 90 & 198 \\
\hline EFA & 119 & 55 & 174 \\
\hline KFBRHSV & 126 & 90 & 216 \\
\hline KFBAHSV & 167 & 103 & 270 \\
\hline KFBAHSZ & 5 & 3 & 8 \\
\hline Summe & 657 & 434 & 1091 \\
\hline
\end{tabular}

\begin{tabular}{|l|c|c|c|c|c|c|}
\hline Fintierstiele & Länder & $\mathbf{F b}$ & $\mathbf{F e}$ & $\mathbf{( F b - F e )}$ & $\begin{array}{c}(\mathbf{F b}- \\
\mathbf{F e})^{2}\end{array}$ & $\begin{array}{c}(\mathbf{F b}-\mathbf{F e})^{2} \\
\mathbf{F e}\end{array}$ \\
\hline EFB & KAZ & 132 & 135,5 & $-3,5$ & 12,25 & 0,09 \\
\hline EFB & UKR & 93 & 89,5 & 3,5 & 12,25 & 0,14 \\
\hline EFR & KAZ & 108 & 119,2 & $-11,2$ & 125,44 & 1,05 \\
\hline EFR & UKR & 90 & 78,8 & 11,2 & 125,44 & 1,59 \\
\hline EFA & KAZ & 119 & 104,8 & 14,2 & 201,64 & 1,92 \\
\hline EFA & UKR & 55 & 69,2 & $-14,2$ & 201,64 & 2,91 \\
\hline KFBRHSV & KAZ & 126 & 130,1 & $-4,1$ & 16,81 & 0,13 \\
\hline KFBRHSV & UKR & 90 & 85,9 & 4,1 & 16,81 & 0,19 \\
\hline KFBAHSV & KAZ & 167 & 162,6 & 4,4 & 19,36 & 0,12 \\
\hline KFBAHSV & UKR & 103 & 107,4 & $-4,4$ & 19,36 & 0,18 \\
\hline KFBAHSZ & KAZ & 5 & 4,8 & 0,2 & 0,04 & 0 \\
\hline KFBAHSZ & UKR & 3 & 3,2 & $-0,2$ & 0,04 & 0,01 \\
\hline Summe & & 1091 & 1091 & 0 & & $\mathbf{X}^{\mathbf{2}=} \mathbf{8 , 3 3}$ \\
\hline
\end{tabular}

Der $X^{2}$ - Wert $=11,1$ signifikant auf dem Niveau von 0,05 (Zweiseitig)

Der $X^{2}$ - Wert $=20,5$ signifikant auf dem Niveau von 0,01 (Zweiseitig) $X^{2}=8,33<11,1, d$. h. das Ergebnis ist nicht signifikant. 
In der Tab. 29 sind die Fintierstile für die Boxer aus Kasachstan und die Fintierstile für die Boxer aus der Ukraine nach der $\mathbf{X}^{2}$ - Test Methode dargestellt. Die kasachischen Boxer verwenden die Fintierstile anders als die ukrainischen Boxer.

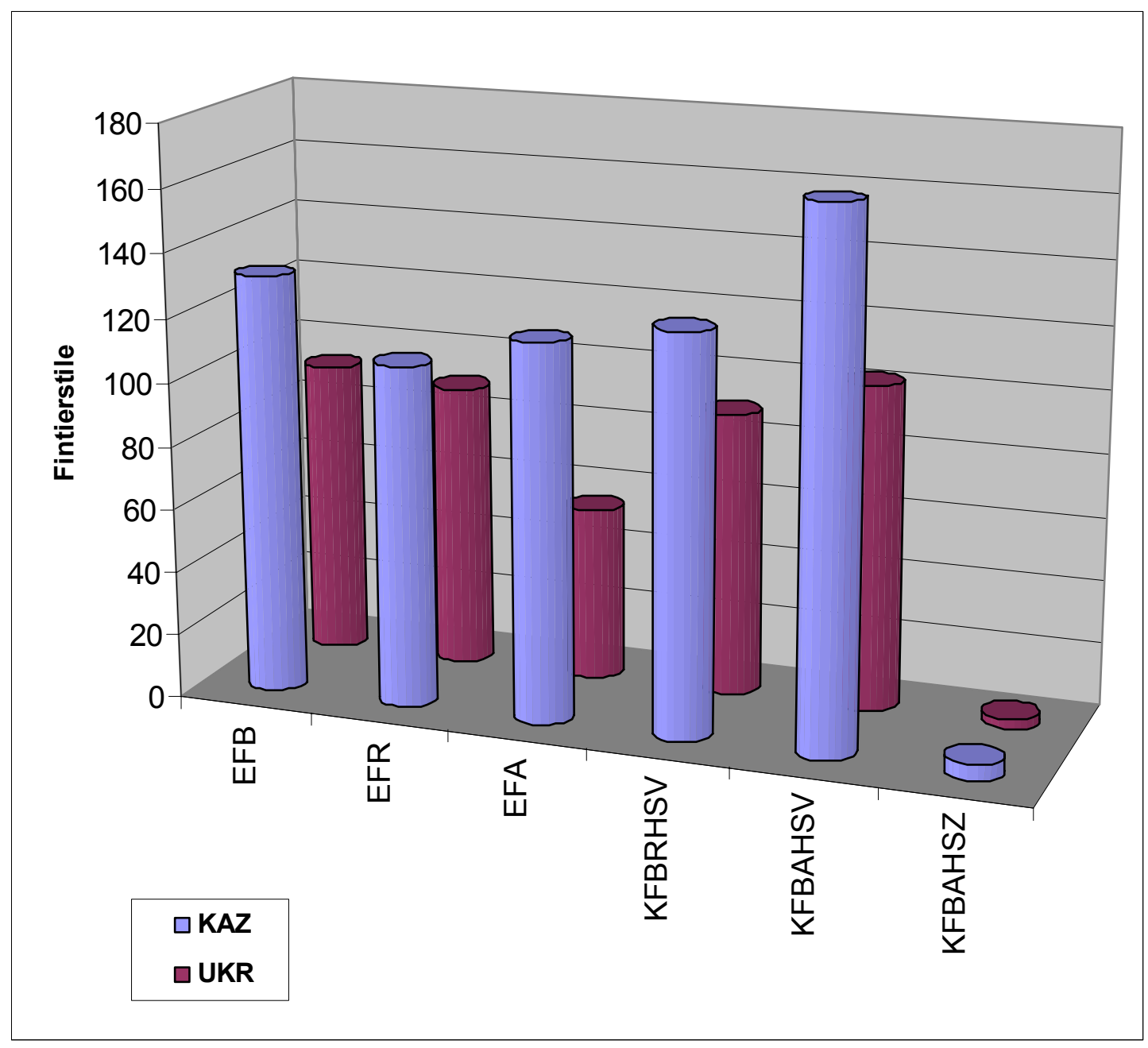

Abb. 66: Die kasachischen $(n=6)$ Fintierstile unterscheiden sich von den ukrainischen $(n=5)$ Fintierstilen.

In der Abb. 66 ist die Häufigkeit der Fintierstile der Boxer aus Kasachstan und der Boxer aus der Ukraine aufgezeigt. Die kasachischen Boxer verwenden die Fintierstile anders an als die ukrainischen Boxer, aber die Unterschiede zu der Ukraine sind statistisch nicht signifikant. 


\section{Diskussion der Ergebnisse}

Bei den Siegern und Verlierern im Boxen zeigen die Ergebnisse die unterschiedlichen Verwendungen von 19 Fintenarten im Finale und Halbfinale.

Tabelle 2 zeigt einen signifikanten Unterschied zwischen dem Sieger und Verlierer bei der Fintenverwendung für den Sieger. Dies ist auch mit einer größeren Anzahl an Schlägen verbunden. LEHMANN (2000) geht von der Einheit des technisch-taktischen und konditionellen Trainings aus. BARTH (2003) fordert für den Boxer eine erhöhte Schnellkraftausdauer. Die Ergebnisse der Analyse zeigen die Berechtigung dieser Forderungen auch für das Fintierverhalten.

Finten sind sehr wichtig, um in einem Wettkampf zu siegen. Sie sind ein Teil des taktischen Wettkampfplans. Ein guter Boxer muss während des gesamten Kampfes fintieren. Der Sieger hat mehr Reaktionsschnelligkeit, Aufmerksamkeit, Superzeichenbildung, Koordination und Antizipation als der Verlierer, weil er die Finten unter den vorbenannten Eigenschaften besser beherrscht.

Der Sieger hat je nach dem Erfahrungsgrad in der Technik des Fintierens mit Partnern, im Schattenboxen und an den Boxgeräten (Sandsack, Plattformbirne, Maisbirne, Doppelendball, Federbirne, Wandpolster und Pratzen) dementsprechend Angriffs und Verteidigungspotenzial. Durch das Fintieren mit den Armen, dem Oberkörper und den Beinen aber auch in der Kombination mit taktischem Handeln erhöhen sich die Chancen auf einen erfolgreichen Kampf.

KÜRZEL (1997) hat gezeigt, dass Fitnessboxen, eine spielerische Variante des Boxens, zur der Steigerung des körperlichen Leistungsvermögen dient. Das Training besteht vorwiegend aus Gymnastikübungen sowie der Einübung von Grundlagen des Boxens (Übungen mit Sandsack, Doppelendball, Maisbirne, Plattformbirne oder mit Partner) in zahlreichen, an das persönliche Leistungsvermögen ausgerichteten Trainingsprogrammen und Übungen. 
FIEDLER (1997) und ELLWANGER; ELLWANGER (1998) haben gezeigt, dass der Boxer die Angriffsdurchführung ausüben kann, nachdem er sich entsprechend seiner taktische Absicht durch die vorbereitenden Handlungen (z. B. Finten) in eine günstige Ausgangsposition im Schlagbereich des Gegners gebracht hat. Der Angriff kann in Abhängigkeit von der aktuellen Kampfsituation und den individuellen Voraussetzungen des Boxers als Einzelschlag, Schlagverbindung, Schlagkombination oder Serie erfolgen. Angriffsvorbereitung und Angriffsabschluss bilden eine Einheit. Der Angreifer sollte Vorbereitungshandlungen und die eigentliche Angriffsführung im Verlauf des Kampfes variieren, um eine mögliche Einstellung des Gegners zu erschweren.

IRANYI (1973) hat gezeigt, dass die Taktik trainierbar und ein wesentliches Element für die Ausbildung eines guten Boxer ist. Voraussetzung zum Taktiktraining ist die Automatisation der boxerischen Handlungen (Technik), d.h. die richtige Reaktion auf einen vom Gegner ausgehenden Reiz, ein Entscheidungsprozess, der jeweils sehr schnell getroffen werden muss. Für das Training bedeutet das, dass der Trainer möglichst viele Reaktionsformen simulieren muss, um das Entscheidungsrepertoire des Boxers zu vergrößern. Der Trainer setzt also die jeweiligen Reize, auf die der Boxer in erster Absicht (direkte Reaktion) oder zweiter Absicht (eigene Initiative des Boxers) ansprechen muss. Als Erklärungsmodell für die Trainingsmethode wird ein kybernetisches System herangezogen, zur speziellen Methodik werden Übungssequenzen vorgestellt.

Diese Forschungsergebnisse geben Gewissheit für die Richtigkeit der ersten Hypothese dieser Untersuchung: Der Sieger verwendet mehr Finten.

Tabelle 3 zeigt einen signifikanten Unterschied zwischen dem Sieger und Verlierer bei der kombinierten Fintenverwendung für den Sieger. Das perfekte Beherrschen der einfachen Finten hilft dem Sieger, um mehr Kombinierte Finten ausführen zu können. Mehr an Kombinierten Finten bedeuten auch eine größere Schlagdichte, aber es sind nicht nur mehr sondern auch entsprechend Kombinierte Schläge. 
Der Sieger hat mehr Bewegungsschnelligkeit, Koordination, Genauigkeit und Konzentrationsfähigkeit als der Verlierer, weil er die kombinierten Finten und andere Faktoren ausnutzt. Der Sieger hat intensiver die kombinierten Finten an den Boxgeräten geübt.

MÜLLER (1991) und SONNENBERG (1993) zeigten, dass es für die Taktikausbildung eines Boxanfängers bedeutend ist, Standardsituationen zu schaffen und diese ständig zu erweitern. Bereits bei Partnerübungen lernt der Boxer, dass Einzelstöße einmalig Treffer bringen. Also müssen Aktionen gestartet, herausgearbeitet, in das Training eingeplant und geübt werden, die nicht voraussehbar und berechenbar sind. Dazu gehört zum Beispiel das Andeuten eines Stoßes, der gar nicht ausgeführt wird, aber den Gegner veranlasst, eine Abwehrbewegung einzuleiten. Derartige Aktionen nennt man Finten.

TYSLER und TYSLER (1995) haben anhand einer Analyse gezeigt, dass die verschiedenen Faktoren beim Boxen eine Gliederung der Taktikmerkmale in Angriff, Verteidigung, Manöver, Position, Schnelligkeit und Abwarten der Kampfführung ergeben. Für diese verschiedenen Faktoren werden taktische Empfehlungen ausgesprochen. Besondere Bedeutung wird der Verwendung von Finten zugesprochen, der Vervollkommnung von Kombinationen aus Angriff und Verteidigung, der Verwendung von Methodiken, die das Handlungsarsenal des Gegners einschränken und eine Konzentration auf die Schaffung bestimmter Situationen fördern. Diese Ergebnisse geben Gewissheit für die Richtigkeit der zweiten Hypothese: Der Sieger verwendet mehr Kombinierte Finten.

Tabelle 4 zeigt einen signifikanten Unterschied zwischen den Siegern und Verlierern bei variabler Kombinierter Fintenverwendung für den Sieger.

Der Boxer muss die Fintenwechsel in gesamtem Kampf mit dem Gegner als Täuschung verwenden, wenn er nur eine oder zwei Kombinierte Fintenarten verwendet, dann wird der Gegner schnell die angewandte Taktik erkennen und den Kampfverlauf zu seinen Gunsten gestalten. Hier besteht die Gefahr, die Runde aber auch den Kampf durch unterlassenes Variieren des Fintierens zu verlieren. Das erfordert vom Boxer mehr Zeit während des Trainings, um die Kombinierten Finten gründlich ausführen zu können. Weil die variablen Kombi- 
nierten Finten ein wichtiger Teil des taktischen Wettkampfplans sind, sind sie vom Boxer während des gesamten Kampfes einzusetzen.

VORONIN (1983) hat gezeigt, dass die Erarbeitung eines individuellen Boxstils, der den besonderen sportlichen Eigenschaften des Boxers entspricht, eine der wichtigsten Möglichkeiten ist, hohe Erfolge zu erzielen. Es wird unterschieden in Angriffs- und Verteidigungskampf und eine aus diesen beiden Stilen Kombinierter Kampftaktik. Der Kampfstil des Boxers wird hauptsächlich durch die Schnelligkeit bestimmt, die sich als Bewegungseigenschaft in der Schnelligkeit einzelner und serieller Bewegungen, in der Reaktionsgeschwindigkeit und in der Reaktion auf ein bewegliches Objekt zeigt. Angriffsboxer haben hierbei eine geringere zentrale Hemmzeit und ein höheres Eigentempo als Verteidigungsboxer. Boxer des Kombinierten Stils sind den Angriffsboxern ähnlicher als den Verteidigungsboxern. Boxern mit ausgeprägten Schnelligkeitsfähigkeiten ist daher der angreifende oder der Kombinierte Kampfstil zu empfehlen. Boxer mit mangelhaft ausgebildeten Schnelligkeitsfähigkeiten dagegen der Verteidigungsstil mit einer gewissen Distanz. Dieses Forschungsergebnis unterstützt die Richtigkeit der dritten Hypothese: Der Sieger verwendet mehr variable Kombinierte Finten als der Verlierer.

Tabellen 5, 6, 7, 8 zeigen einen signifikanten Unterschied zwischen schweren Boxern und leichten Boxern bei der Fintenverwendung. Die Wahrscheinlichkeit eines K.O. in einem Boxkampf ist für schwere Boxer höher als für leichte Boxer. Deshalb ist es für den schweren Boxer von äußerster Notwendigkeit, alle Stile und Variationen von Finten während des Kampfes zu benutzen, um nicht den Kampf frühzeitig durch K.O. zu verlieren. Der schwere Boxer hat weniger Beweglichkeit als der leichtere. Im Gegensatz zu dem leichten Boxer kann er nicht so viele Bewegungsabläufe in dem Ring leisten und ist daher auf das vermehrte Verwenden von Finten in Vorbereitung des Angriffs, Gegenangriffs und der Verteidigung angewiesen, um den Wettkampf zu gewinnen. Dieses Forschungsergebnis steht für die Richtigkeit der vierten Hypothese: Schwere Boxer verwenden Finten häufiger als leichter Boxer. 
Tabelle 9, 10, 11, 12 zeigen einen signifikanten Unterschied zwischen schweren Boxern und leichten Boxern bei Kombinierter Fintenverwendung wobei schwere Boxer mehr Kombinierte Finten verwenden. Da der schwere Boxer die einfachen Finten gründlich beherrscht, kann er auch dementsprechend die Kombinierten Finten ausführen. Wer einfache Finten häufiger verwendet, wird auch Kombinierte Finten häufiger verwenden. Dieses Forschungsergebnis zeigt die Richtigkeit der fünften Hypothese: Schwere Boxer verwenden Kombinierte Finten häufiger als leichter Boxer.

Tabelle 13 zeigt einen nicht signifikanten Unterschied zwischen dem Sieger und dem Verlierer bei gesteigerter variabeler Fintenverwendung in der 3. und 4 . Runde. Der Sieger wird in den ersten Runden das Fintierverhalten des Gegners studieren, um so für die letzten Runden seine Finten dem gegnerischen Verhalten anzupassen und so zu variieren, dass der Gegner keine Möglichkeit hat anzugreifen.

ADREEV (1986) hat gezeigt, dass die Gruppenform des Trainings ungleiche Wirkungen auf jeden einzelnen Boxer hat, besonders bei individuellen Sportarten, zu dem das Boxen gehört. Das individuelle Training ist jedoch für Boxer unablässig. Für die individuelle Vorbereitung des Boxers bedarf es einer komplexen Information des Trainers über den Boxer, seine Umgebung und seiner Gegner. Die Boxer müssen bei der Analyse der Training- und Wettkampfleistung sowie bei der Planung der bevorstehenden Vorbereitung miteinbezogen werden. Die sportliche Vorbereitung von Boxern beinhaltet folgende Aspekte: Theoretische, erzieherische, psychologische, taktische, technische und physische. Um den Boxer gezielt motivieren zu können, müssen seine schwachen Seiten erkannt, seine individuellen Unzulänglichkeiten beseitigt werden. Die Tendenz der korrigierenden Arbeit während des Trainingsprozesses muss sein, die schwächeren Eigenschaften an das Niveau der guten anzugleichen. Die individuelle Vorbereitung des Boxers muss sorgfältig geplant, organisiert und durchgeführt werden.

Diese nicht signifikanten Forschungsergebnisse gelten der sechsten Hypothese: Der Sieger macht gegen Ende des Kampfes häufiger variable Finten als der Verlierer. 
Tabelle 14, 15 zeigen einen nicht signifikanten Unterschied zwischen den schweren Siegern und den leichten Siegern, schweren Boxern und leichten Boxern bei variabeler Fintenverwendung gegen Ende des Kampfs.

Schwere Sieger werden sich in den ersten Runden des Kampfes mit geringfügig höherem Fintieren an den Gegner anpassen, um so für die letzten Runden ihre Finten optimal zu erhöhen, sodass der Gegner keine Möglichkeit hat in der Endphase wirkungsvoll anzugreifen oder sogar einen K.O. Treffer zu platzieren. Diese nicht signifikanten Forschungsergebnisse gelten der Hypothese zu 6.1 und 6.2:

1- Der Schwere Sieger macht gegen Ende des Kampfes häufiger variable Finten als der leichte Sieger, aber die Unterschiede sind statistisch nicht signifikant.

\section{2- Der Schwere Boxer macht gegen Ende des Kampfes häufiger variable}

Finten als der leichte Boxer, aber die Unterschiede sind statistisch nicht signifikant. Es bleibt einer zukünftigen Arbeit vorbehalten zu überprüfen, ob bei der Beobachtung von mehr Kämpfen die Unterschiede signifikant werden.

Tabelle 16, 17, 18, 19 zeigen einen signifikanten Unterschied zwischen dem Sieger und dem Verlierer beim Variieren der Art der Finten in den jeweiligen Runden. Der gute Boxer versucht ab der ersten Runde die Art der Finten zu variieren, um dem Gegner seine Taktik nicht darzulegen. Deshalb führt er während des gesamten Kampfes die Variation dieser Finten durch, um jederzeit einen erfolgreichen Angriff vorbereiten zu können.

Der Sieger entdeckt von der ersten Runde an die Merkmale und Fehler des Gegners, daher kann er entscheiden, welche passende Fintenart, welche passende Verteidigung gegen die Schläge des Gegners in jeder Runde auszuführen sind. Der Verlierer kann die Art der Finten von Runde zu Runde nicht dementsprechend angepasst variieren. Der Sieger trainiert die Art der Finten mit unterschiedlichen Partnern während der Vorbereitung der Wettkampfperiode häufiger als der Verlierer, deshalb kann er während des gesamten Kampfes die Art der Finten stärker als der Verlierer variieren. 
Diese Forschungsergebnisse sind die Grundlage der siebte Hypothese: Die Sieger variieren die Art der Finten in den Runden stärker als die Verlierer.

Tabelle 20, 21, 22, 23, 24, 25, 26, 27, 28, 29 zeigen einen großen signifikanten Unterschied zwischen den nationalen Boxschulen aus den USA, Russland, der Ukraine, Kuba und Kasachstan in den sechs Fintierstilen.

Neben individuellen Stilen und typischen Fintierstilen für alle Gewichtsklassen gibt es auch nationale Kampfstile, die sich in Art und Häufigkeit der Verwendung von Finten unterscheiden.

Diese Forschungsergebnisse entsprechen der 8.1, 8.2, 8.3 und 8.4 Hypothese: Es gibt erfolgreiche nationale Boxschulen, die sich mit einem eigenen Fintierstil darstellen. Die Boxer aus USA, Russland, der Ukraine, Kuba und Kasachstan unterscheiden sich in ihren Fintiermustern.

1. Die Amerikanische Boxschule unterscheidet sich von denen aus RUS, UKR, CUB und KAZ.

2. Die RUS Boxschule unterscheidet sich von der aus UKR, CUB und KAZ.

3. Die CUB Boxschule unterscheidet sich von der aus der UKR und KAZ.

4. Die KAZ Boxschule unterscheidet sich nicht von der aus der UKR.

Durch die Dominanz von Nationaltrainern und Trainingszentren werden bestimmte Fintierstile vermittelt. Kenntnisse solcher nationalen Fintierstile sind von Bedeutung, um sich z. B. bei Boxländerkämpfen auf bis dahin selten beobachtete Gegner einstellen zu können. Die Ergebnisse überraschen etwas, da sie dem Vorurteil widersprechen, dass es noch immer einen „,Ostblock-Stil” gäbe, dem ein ,,amerikanischer" gegenüberstehe. Es zeigt sich aber, dass es zehn Jahre nach Untergang des Ostblocks ausgeprägte nationale Boxschulen zumindest in den Fintierstilen gibt. Allerdings sind keine signifikanten Unterschiede der Fintierstile zwischen Kasachstan und der Ukraine messbar, was doch noch auf die gemeinsame Herkunft der Boxschulen verweisen könnte. 


\section{Schlussfolgerungen und Nachbetrachtungen}

\subsection{Schlussfolgerungen}

Die Ergebnisse der Analyse lassen die folgenden Schlussfolgerungen zu:

> Auch ohne Analyse der Finten mit den Augen lassen sich Finten analysieren.

> Das vom Verfasser angewandte Analysesystem der Finten eignet sich, um signifikante Unterschiede zu ermitteln.

$>$ Finten sehr sind wichtig, um in einem Wettkampf zu siegen. Sie sind Teil des taktischen Wettkampfplans.

> Sieger verwenden die Finten mehr als Verlierer während des gesamten Kampfes.

> Sieger verwenden die Kombinierten Finten mehr als Verlierer während des gesamten Kampfes.

$>$ Sieger beherrschen die Kombinierten Finten, deshalb können sie auch mehr variable kombinierte Finten ausführen.

> Schwere Boxer verwenden alle Stile von Finten und Kombinierte Finten häufiger als leichter Boxer, um K.O. Schläge zu vermeiden.

$>$ Sieger verwenden häufiger variable Finten gegen Ende des Kampfes als der Verlierer.

$>$ Schwere Sieger und schwere Boxer können häufiger variable Finten gegen Ende des Kampfes verwenden als leichte.

$>$ Sieger variieren die Art der Finten von der ersten Runde bis zum Ende des Kampfes stärker als Verlierer.

$>$ Es gibt einen großen signifikanten Unterschied zwischen den meisten nationalen Boxschulen. 


\subsection{Nachbetrachtungen}

Aufgrund meiner Ergebnisse komme ich zu folgenden Empfehlungen:

$>$ Die Amateurboxverbände der jeweiligen Länder sollten ihre Aufmerksamkeit auch auf Finten im Trainingsprogramm lenken und mit der Notwendigkeit und Sorgfalt die Vorbereitung des Trainers für die Ausführung des Trainingprogramms der Finten unterstützen.

$>$ Der geringe finanzielle Aufwand zur Wettkampfanalyse liefert genügend Aussagen zu Finten mit dem Körper. Bestimmte Finten, z. B. solche in Kombination mit den Augen (Blickrichtung), lassen sich aber nur durch Verbesserung des Analysematerials beobachten. Empfehlenswert wären eine höhere Bild- und Zeitauflösung sowie in den Ecken des Ringes positionierte Kameras. Gerade in Hinblick auf die Finten mit den Augen wäre es überlegenswert, für das Training eine kleine Kamera an der Stirn des Trainingspartners zu montieren, um die Bedeutung der Finten mit den Augen bei Anfängern, Fortgeschrittenen und Olympiateilnehmern erfassen zu können. Weitere Beobachtungsmethoden wären unter großem finanziellem und technischem Aufwand denkbar.

$>$ Eine gesonderte Untersuchung des kubanischen Fintierstil wäre lohnenswert, da die kubanischen Boxer die meisten Finten einsetzten und am erfolgreichsten waren.

Durch Befragung der Boxer und gezielte Analyse des Videomaterials wäre vielleicht noch ein Ausbau der Fintenklassifizierung möglich. 


\section{Zusammenfassung}

\section{- Problemstellung}

Die Entwicklung des Boxens in den letzten zehn Jahren ist vor allem auf Änderungen zurückzuführen, die auf den Einsatz verschiedener technischer und taktischer Mittel sowie modifizierter Regelauslegungen zurückgehen. Ausgewertet wurden die Finalkämpfe der Olympischen Spiele 1988, die Weltmeisterschaften 1993 und die Olympischen Spiele 1996. Hervorgehoben wurde die steigende Anzahl von Verteidigungshandlungen. Mit der Einführung des elektronischen Kampfrichterwesens gibt es auch Veränderungen in der Taktik der Kampfesführung. Die Zahl der eingesetzten taktischen Varianten der Kampfentwicklung ist spürbar zurückgegangen, es wird auf ein Übergewicht in der Punktwertung mit Hilfe einzelner Schläge zu Beginn des Kampfes hingearbeitet. Insgesamt kann festgestellt werden, dass die Modernisierung der Technik zur höheren Sicherheit für die Boxer geführt hat. Ein Vergleich der K.O. Siege von 1988 und 1996 (28 zu 4) legt eindeutig Zeugnis ab vom Wandel im modernen Boxen.

Die Finten sind eine Form der Boxtechnik und- taktik. Durch das Fintieren erfährt der Boxer viel über die Reaktionen, die Fertigkeit und das Kampfniveau seines Gegners. Daraufhin ist er in der Lage, den Angriffsschlag auszuführen. Das Fintieren ist in jeder Runde in all seinen Variationen variabel zu handhaben und ermöglicht eine Vielfalt von Reaktionsmöglichkeiten und ist somit eine der wesentlichen Vorraussetzungen, die der Boxer beherrschen muss, um den Erfolgssangriff zu ermöglichen.

Ich habe die Olympischen Spiele Sydney 2000 ausgewählt, weil sie die letzten Olympischen Spiele bis heute sind. Außerdem nehmen viele der besten Boxer der Welt an den Olympischen Spielen teil. Deshalb habe ich bei den Olympischen Boxwettkämpfen in Sydney im Jahre 2000 die einfachen und kombinierten Finten analysiert, um Aufschluss über die Art der von den Siegern beherrschten Finten zu erhalten und zu wissen, welche Arten von Finten am wirksamsten sind. 
Techniken wurden bereits wissenschaftlich untersucht, aber gegenwärtig liegen nur wenige Untersuchungen von Techniken und Taktiken beim Boxen vor.

Der Boxkampf und das Fintieren als Strategie im Boxen wurden bis heute noch nicht ausführlich empirisch untersucht. Aus diesem Grund wurden im ersten Teil der Arbeit bisher erforschte Erkenntnisse zu Täuschungshandlungen im Sport angeführt und die Bedeutung von Finten im Sport dementsprechend eingeordnet. Der zweite Teil der Arbeit beinhaltet schließlich die Analyse von Amateurboxern aller Klassen in Hinsicht auf ihr Fintier-Verhalten.

\section{- Zielsetzung}

Die vorliegende Studie sollte uns folgende Fragen beantworten:

1- Welche Arten von Finten beherrschen die Sieger?

2- Welche Finten sind am wirksamsten?

3- Welche Gewichtsklassen verwenden Finten häufiger?

4- Welche Arten von Finten dominieren in den Runden?

5- Welche nationalen Boxschulen sind erfolgreich?

6- Wie können unsere Boxer und die Finalboxer der Meisterschaft charakterisiert werden?

\section{- Inhaltliche Hypothesen}

1. Der Sieger verwendet mehr Finten als der Verlierer.

2. Der Sieger verwendet mehr Kombinierte Finten als der Verlierer.

3. Der Sieger verwendet mehr variable Kombinierte Finten als der Verlierer.

4. Schwere Boxer verwenden Finten häufiger als leichte Boxer.

5. Schwere Boxer verwenden Kombinierte Finten häufiger als leichte Boxer.

6. Der Sieger macht gegen Ende des Kampfes häufiger variable Finten als der Verlierer.

6.1 Schwere Sieger machen gegen Ende des Kampfes häufiger variable Finten als leichte Sieger.

6.2 Schwere Boxer machen gegen Ende des Kampfes häufiger variable Finten als leichte Boxer. 
7. Die Sieger variieren die Art der Finten von Runde zu Runde stärker als die Verlierer.

8. Es gibt erfolgreiche nationale Boxschulen, die sich mit einem eigenen Fintierstil darstellen. Die Boxer aus den USA, Russland, der Ukraine, Kasachstan und Kuba unterscheiden sich in ihren Fintier-Mustern.

8.1. Die Amerikanische Boxschule unterscheidet sich von des russischen, der ukrainischen, der kubanischen und der kasachischen.

8.2. Die russische Boxschule unterscheidet sich von der ukrainischen, der kubanischen und der kasachischen.

8.3. Die kubanische Boxschule unterscheidet sich von der ukrainischen und der kasachischen.

8.4. Die kasachische Boxschule unterscheidet sich von der ukrainischen.

\section{- Untersuchungsmethode}

- Auswahl des Stichprobe

Am Boxen bei den Olympischen Spielen in Sydney 2000 nahmen 75 Länder mit 310 Boxern teil. Im Halbfinale waren 22 Länder mit 48 Boxern, im Finale 12 Länder mit 24 Boxer vertreten.

Ich habe in der Arbeit 16 Kämpfe der Halbfinale und 12 Finalkämpfe der Olympischen Spiele in Sydney analysiert.

\section{- Konzeption und Auswertung der Kämpfe}

Ich habe ein Analysesystem aufgebaut, das 19 verschiedene Arten von Finten beim Boxen unterschied. Danach habe ich diese 6 Analysesysteme für die Fintenstile für jeden Kampf zusammengefasst, um die Analyse besser handhaben zu können.

Zuerst habe ich die Finten mit dem Videorecorder registriert, nachher habe ich die Kämpfe von den Videokassetten auf dem Computer gespeichert, um die Kämpfe damit zu analysieren. Mit dem Win DVD Programm konnte ich die Kämpfe mit verschiedenen Geschwindigkeiten sehen und die einfachen Finten analysieren. 
Für die Forschung ist es nun wichtig, die Objektivität der Analysen zu bestimmen. Hierzu musste ein weiterer Experte das Analysesystem ,lernen" und auf die Kämpfe anwenden. Durch den Vergleich von dessen Ergebnissen mit meinen konnte ich dann einen Objektivitätskoeffizienten berechnen. Deshalb habe ich die fünf Kämpfe des Viertelfinales analysiert und ein erfahrener Boxtrainer aus Armenien hat dieselben Kämpfe analysiert. Danach habe ich die Objektivitätskoeffizienten berechnet, er betrug $R=0,99$, d.h. mein Analyseverfahren hat eine überaus große Untersucherobjektivität.

\section{- Reliabilität}

Im Hinblick auf die Genauigkeit der Messung ist zum einen die Anzahl der Fernsehkameras, mit denen die Bilder aufgenommen wurden, von Bedeutung. Die Boxkämpfe wurden in der Regel von fünf Kameras aufgenommen, die in den Positionen A, B, C, D und aus der Vogelperspektive (vgl. Abb. 39) standen. Hierdurch war es fast immer möglich, beide Kämpfer in einer Weise auf dem Bildschirm zu sehen, dass die Finten beider gut analysiert werden konnten. Allerdings gab es zwischendurch auch immer wieder Momente, in denen dies nicht der Fall war. Da sich die Kämpfer nicht immer in einer Position befanden, die eine lückenlose Analyse zuließ. Bei durchschnittlich 0,307 \% der Zeit ohne richtige Bilder für die Analyse der Finten können die Daten als sehr reliabel bezeichnet werden.

\section{- Validität}

Da Finten analysiert wurden, ist die Arbeit logisch valid. Allerdings konnten aufgrund der Fernsehbilder nicht alle Finten analysiert werden. Die Finten mit den Augen konnten nicht in die Analyse einbezogen werden. Hierdurch fehlen drei von 22 möglichen Fintenarten, sodass 19 verschiedene Finten in die Analyse einbezogen wurden. Zwar sind diese Finten mit den Augen sehr wichtig, da sie parallel und additiv zu den anderen Finten einsetzbar sind, aber auf der anderen Seite sind es eben nur 17,6 \% der möglichen Fintenarten. Insgesamt kann die Analyse somit noch als valid gelten. Man sollte sich jedoch immer daran erinnern, dass eine wichtige Fintenart fehlt. 


\section{- Objektivität}

Für die Forschung ist es nun wichtig, die Objektivität der Analysen zu bestimmen. Für die Auswerterobjektivität muss neben mir noch ein weiterer Experte das Analysesystem ,Iernen" und auf die Kämpfe anwenden. Damit kann ich dann einen Objektivitätskoeffizienten berechnen. Deshalb habe ich 5 Kämpfe der Viertelfinale zu je vier Runden à zwei Minuten analysiert. Der Boxtrainer GEGHAM HAKOBIAN aus Armenien hat dieselben fünf Kämpfe unabhängig von mir analysiert. Er war zum Zeitpunkt der Analyse zu Studienzwecken in Göttingen und hat dieselben Videogeräte benutzt. Ich habe ihm in mein FintenAnalyse-System vier Stunden lang eingewiesen. Dann hat er unabhängig von Untersucher selbständig die Analyse des Videomaterials der fünf Kämpfe durchgeführt (25 Stunden). Die Gesamtzahl der Finten habe ich in die Rangfolgen 1 und 2 übertragen. Der Rangkorrelationskoeffizient beträgt $R=0,991$, d.h. es besteht eine sehr hohe Objektivität bezogen auf das analysierte Material.

\section{- Statistische Auswertung}

Die statistische Auswertung der Daten erfolgte im Einzelnen manuell. Es handelte sich um folgende Maße und Verfahren:

Ich verwendete die folgenden Koeffizienten:

1.Das arithmetische Mittel, Mittelwert

2.Den WILCOXON- Test

3. Chi-Quadrat-Test ( $x^{2}-$ Test)

4.Rangkorrelationskoeffizienten.

\section{- Schlussfolgerungen}

Die Ergebnisse der Analyse zeigen die folgenden wichtigen Schlussfolgerungen:

$>$ Finten sehr sind wichtig, um in einem Wettkampf zu siegen. Sie sind Teil des taktischen Wettkampfplans.

> Sieger verwenden häufiger die kombinierten Finten als Verlierer während des gesamten Kampfes. 
> Sieger beherrschen die Kombinierten Finten, deshalb können sie auch mehr variable Kombinierte Finten ausführen.

$>$ Schwere Boxer verwenden alle Stile von Finten und Kombinierte Finten häufiger als leichter Boxer, um K.O. Schläge zu vermeiden.

$>$ Schwere Sieger und schwere Boxer können häufiger variable Finten gegen Ende des Kampfes verwenden als leichte.

$>$ Sieger variieren die Art der Finten von der ersten Runde bis zum Ende des Kampfes stärker als Verlierer.

$>$ Es gibt einen großen signifikanten Unterschied zwischen den meisten nationalen Boxschulen.

\section{- Nachbetrachtungen}

Von den wichtigen Schlussfolgerungen komme ich in meinem Analyserahmen zu folgenden Empfehlungen:

$>$ Der geringe finanzielle Aufwand zur Wettkampfanalyse liefert genügend Aussagen zu Finten mit dem Körper. Bestimmte Finten, z. B. solche in Kombination mit den Augen (Blickrichtung), lassen sich aber nur durch Verbesserung des Analysematerials beobachten. Empfehlenswert wären eine höhere Bild- und Zeitauflösung sowie in den Ecken des Ringes positionierte Kameras. Gerade in Hinblick auf die Finten mit den Augen wäre es überlegenswert, für das Training eine kleine Kamera an der Stirn des Trainingspartners zu montieren, um die Bedeutung der Finten mit den Augen bei Anfängern, Fortgeschrittenen und Olympiateilnehmern erfassen zu können. Weitere Beobachtungsmethoden wären unter großem finanziellem und technischem Aufwand denkbar.

$>$ Eine gesonderte Untersuchung des kubanischen Fintierstil wäre lohnenswert, da die kubanischen Boxer die meisten Finten einsetzten und am erfolgreichsten waren.

Durch Befragung der Boxer und gezielte Analyse des Videomaterials wäre ein Ausbau der Fintenklassifizierung möglich. 


\section{Literaturverzeichnis}

ACKERMAN, P. L., (1987): Individual differences in skill learning. An integration of psychometric and information processing perspectives, Psychological Bulletin, 102 (1), 3-27.

ACKERMAN, P. L., (1989): Individual differences and skill acquisition. In: ACKERMAN, P. L; STERNBERG, R. J. u. a. (Hrsg.): Learning and individual differences. New York, 165-217.

ACKERMAN, P. L., (1990): Correlational analysis of skill specifity: Learning, abilities, and individual differences, J. of exper. Psychological. Learn, 16 (5), 883-901.

ADREEV, T., (1986): Individuelle Vorbereitung im Boxen. Vapr. na fiz. Kult, 31 (9), 58-62. (Abstract auf deutsch in Spolit)

ALBRECHT, D. G., (1982) (Hrsg.): Recognition of Pattern and Form: Proceedings of a Conference held at the University of Texas at Austin. New York.

ALICHANOV, I. I. ; SACHMURADOV, J. A., (1985): Die Taktik im Freistilringen. In: PREOBRAZENSKIJ, S. A. (Hrsg.), Sportivnaja borba (Ringen), 30-33. (Abstract auf deutsch in Spolit)

ALLPORT, D. A., (1980): Attention and Performance. In: CLAXTON, G. (Hrsg.): Cognitive Psychology. London, 112-153.

AMBRUS, A. ; BÖHMER, D. u. a., (1981): Boxsport. In: Sporttraumatologie, PFÖRRINGER, W. ; ROSEMEYER, B. u. a. Erlangen, 72-79.

ANFREEV, T., (1996): Das bulgarische Boxen wird in Atlanta 96 sein hohes Ansehen verteidigen. Sport i nauka, 40 (5), 86-92. (Abstract auf deutsch in Spolit) 
ARSLANJAN, M. G., (1978): Einige Fragen des Grundlagentrainings von 1213 jährigen Jugendlichen im Boxen. Teor. Prakt. fiz. Kult, 41 (4), 39-40. (Abstract auf deutsch in Spolit)

BALLREICH, R., (1970): Grundlagen sportmotorischer Tests. Frankfurt am Main.

BARISCH, E., (1953): Unser Boxlehrbuch. Berlin.

BARTONIETZ, K., (1996): Biomechanics of the Snatch: Toward a Higher Training Efficiency. Strength and Conditioning, 18 (3), 27-36.

BARTONIETZ, K., (1999): Das vermeintliche Ende der „Periodisierung“ oder Ansätze zur Weiterentwicklung des Trainings. Leistungssport, 29 (1),12-24.

BARTH, B., (1990): Taktik und Fair play. Theorie und Praxis der Körperkultur, 39 (4), 213-219.

BARTH, B., (1991): Taktik und Fair play. Leipziger sportwiss. Beitr, 32 (1), 3445.

BARTH, B., (1995): Wettkampfvorbereitung durch komplexes strategischtaktisches Training. Leistungssport 25 (1), 20-27.

BARTH, B., (2003): Strategisch-taktisches Training in den Zweikampfsportarten im Lichte verschiedener Betrachtungsmodelle. In: SCHUMANN, K.; GARCIA, R. (Hrsg.): Die Entwicklung der Trainingssystems in den Gruppen der Sportarten (= Sport. Leistung. Persönlichkeit, Bd. 4). Schkeuditz, 25-34.

BARTH, B. ; KIRCHGÄSSNER, H., (1984): Ansätze zur Analyse des Zweikampfverhaltens. Theorie und Praxis der Körperkultur, 33 (7), 526-533.

BARTLETT, F.C., (1958): Thinking. London. 
BASTIAN, M., (1978): Trainingsbedingte Anpassungsreaktionen des kardiopulmonalen System bei jungen Boxsportlern. Med. u. Sport,18 (6), 189-196.

BASTIAN, M. ; FIEDLER, H., (1987): Positionen und Lösungsansätze zur Erhöhung der Wirksamkeit des Trainingsprozesses - dargelegt an der Sportart Boxen. Theorie und Praxis Leistungssport, 25 (5), 40-48.

BASTIAN, M., (2003): Erfahrungen bei der Nutzung einer Barokammer für dien konditionelle Vorbereitung von Nachwuchsboxern. In: SCHUMANN, K.; GARCIA, R. (Hrsg.): Die Entwicklung der Trainingssystems in den Gruppen der Sportarten (= Sport. Leistung. Persönlichkeit, Bd. 4). Schkeuditz, 45-52.

BASTIAN, M. ; KIRCHGÄSSNER, H., (1980): Zur Erhöhung des Nivaus der technisch-taktischen Ausbildung im Aufbautraining der Sportart Boxen. Theorie und Praxis Leistungssport, 18 (3), 30-40.

BASTIAN, M. ; KIRCHGÄSSNER, H. u. a., (1982): Ausgewählte Ergebnisse bei der Gestaltung der technisch-taktischen Ausbildung im Aufbautraining der Sportarten Boxen. Theorie und Praxis Leistungssport, 20 (11), 41-53.

BECK, E., (1978): Tauberbischofsheimer Fechtlexikon. Berlin.

BECKER, J., (1979): Der Boxer. Frankfurt am Main.

BERGER, J., (1996): Boxen. Beseitigung von Bewegungs- und Handlungsfehlern. Sportpraxis, Wiesbaden, 37 (5), 46-49.

BÖDEKER, H., (1992): Einführung in die medizinische Statistik. Giessen.

BORTZ, J., (1979): Lehrbuch der Statistik für Sozialwissenschafter. Berlin.

BORTZ, J., (1993): Statistik für Sozialwissenschaftler. 4 Bände, Berlin.

BORTZ, J., (1999): Statistik für Sozialwissenschaftler. Berlin. 
BÖS, K., (1986): Statistikkurs I. Einführung in die Statistik. 3. Auflage, Ahrenburg.

BÖS, K., (2001): Handbuch sportmotorischer Tests. Göttingen.

BÖS, K., (2003): Finte. In: RÖTHIG, P. (Hrsg.): Sportwissenschaftliches Lexikon. 7. Auflage, Schorndorf, 199-200.

BÖTTICHER, H., (1952): 3 Runden. Berlin.

BÖTTCHER, J. ; DEUTSCHER, E., (1999): Biomechanische Ergebnisse zur Bewegungstechnik im Gewichtheben (Reißen). Leistungssport, 29 (4), 57-69.

BÖTTCHER, J. ; DEUTSCHER, E., (2000): Technikübersicht Reißen. OSPBerlin/ BVDG, das in der Athletik abgebildete Technikblatt als Bilddatei über den OSP- Berlin oder den BSP-Berlin bezogen werden.

BOWYER, .J. B., (1982): Box- und Sportgeschichten. Frankfurt am Main.

BRANDT, B. ; STAHL, S., (1984): Vermittlung von Taktik durch Lernprogramme. Konkretisiert an einem Beispiel: Der Gegenblock - ein Lern- und Trainingsprogramm. Basketball, 12 (14), 17-18.

BRAUNE, TH., (1995): Henry Maske - Durchgeboxt. Berlin.

BRAUSKE, H. J. ; SCHLIMPER, L., (1988): Auswertung von Ergebnissen der XI. Kinder- und Jugendspartakiade 1987 im Boxen. Theorie und Praxis Leistungssport, 26 (2/3), 131-136.

BRECHBÜHL, J., (1990): Wann sollen sich Anfänger mit Taktik beschäftigen. Tennis-Sport, 1 (5), 20-21.

BREMERMANN, H., (1976): Pattern Recognition by Deformable Prototypes. In: HILTON, P. (Hrsg.): Structural Stability, the Theory of Catastrophes, and Applications in the Sciences. Lecture Notes in Mathematics, 52 (5), 15-57. 
BRODY, B. E. ; HATFILD, B. D. u. a., (1988): Generalization of self-efficacy to a continuum of stressors upon mastery a high risk sport skill. Journal of Sport and Exercise Psychology, 10 (1), 32-44.

CARL, K., (1983): Training und Trainingslehre in Deutschland. Schorndorf.

CARL, K., (1984): Trainingswissenschaft. In: CARL, D. ; KAYSER, D. u. a. (Hrsg.): Handbuch Sport, Band. 1. Düsseldorf, 135-164.

CHARNAS, L. ; PYERITYR, E., (1986): Neurologic injuries in Boxers. Hosp. Pract, 21 (5), 34-39.

CHISHOLM, R.M ; FEEHAN, T.D., (1977): The Intent to Deceive. In: The Journal of Philosophy, 74 (3), 143-159.

CLAUß, G. ; EBNER, H., (1972): Grundlagen der Statistik für Psychologen, Pädagogen und Soziologen. Frankfurt.

CRATTY, B.J., (1975): Motorisches Lernen und Bewegungsverhalten. Frankfurt.

DAUGS, R., (1988): Zur Optimierung des Techniktrainings durch FeedbackTechnologien. In: MECHLING, H. ; SCHIFFER, J. u. a. (Hrsg.): Theorie und Praxis des Techniktrainings (Hearing des Bundesinstituts für Sportwissenschaft vom 3.-4. Dezember 1986 in Köln). Köln, 124-146.

DENZ, D., (1997): Boxen verständlich gemacht. München.

DERWIN, B., (1990): Sports Performance: The Snatch: Technical Description and Periodization Program. National Strength and Conditioning Association Journal, 12 (2), 7-16. 
DITTRICH, W. ; FRANZ, B. u. a., (1989): Grundsätze, Richtungen und Beispiele der trainingswissenschaftlichen Nutzung von Rechentechnik und Informatik als Faktor zu Effektivitätssteigerung des Trainings aus der Sicht der internationalen Literatur. In: Leipzig, ZFW, (Hrsg.): Körperkultur und Sport, Thematische Information, 70-121.

DERENNE, C. ; HETZLER, R. u. a., (1996): Effects of Training Frequency on Strength Maintenance in Pubescent Baseball Players. Journal of Strength and Conditioning Research, 10 (1), 9-15.

DÖRNER, D. ; KREUZIG, H. W. u. a., (1983) (Hrsg.): Lohhausen: Vom Umgang mit Unbestimmtheit und Komplexität. Bern.

DURJASZ, D. ; NOWAK, T., (1997): Boxen in den 90er Jahren - Veränderungen im Kampfbild. Sport wyczynowy, 34 (1/2),10-12. (Abstract auf deutsch in Spolit)

EBERSPÄCHER, H., (1990): Mentale Trainingsformen in der Praxis. Oberhaching.

ECCLES, J.C., (1985): Willkürmotorik. In, POPPER, K ; ECCLES, J.C (Hrsg.): Das Ich und sein Gehirn. München, 337-358.

ELLWANGER, S. ; ELWANGER, U., (1998): Boxen. Basics. Stuttgart.

ELLWANGER, S. ; HARTISCH, W., (1980): Zur Gestaltung des Lehrgangs Boxen in den Klassen 9 und 10. Körpererziehung, 30 (11), 518-533.

FAIGENBAUM, A. ; POLAKWSKI, C., (1999): Olympic-Style Weightlifting, Kid Style. National Strength Conditioning Association, 21 (3), 75-79.

FALKENBERG, G., (1982): Lügen. Grundzüge einer Theorie sprachlicher Täuschung. Tübingen, 71-98. 
FARRELL, J. E., (1975): The classification of physical education skills. Quest, 24 (1), 63-68.

FELTZ, D. L ; LANDERS, D. M., (1983): The effects of mental practice on motor skill learning and performance- A meta-analysis. Journal of Sport Psychology, 5 (1), 25-57.

FETZ, F., (1980): Bewegungslehre der Leibesübungen. Bad Homburg.

FIEDLER, H., (1974): Boxen. Eine Anleitung für die Ausbildung der Anfänger. Berlin.

FIEDLER, H., (1976): Boxsport. Berlin.

FIEDLER, H., (1989a): Ergebnisse von Beobachtungen im Boxsport bei den Olympischen Sommerspielen 1988 in Seoul. Theorie und Praxis Leistungssport, 27 (5), 70-83.

FIEDLER, H., (1989b): Tendenzen und Aspekte für die Weiterentwicklung des Trainings im Boxsport unter Beachtung des Messplatztrainings. Theorie und Praxis Leistungssport, 27 (1), 18-30.

FIEDLER, H., (1997): Boxen für Einsteiger. 2- Aufl., Berlin.

FIEDLER, H. ; SCHULZ, R., (1990): Zur Leistungsentwicklung im Boxsport Ergebnisse von Beobachtungen bei den WM 1989 in Moskau. Training und Wettkampf, 28 (2/3), 233-244.

FIEDLER, H. ; WAGNER, H. u. a., (1987): Tendenzen der Leistungsentwicklung im internationalen Boxsport. Theorie und Praxis Leistungssport, 25 (1), 101-112.

FINLEY, M ; PLEKET, H., (1976): Die Olympischen Spiele der Antike. Tübingen. 
FLEISHMAN, E. A., (1967): Individual differences in motor learning. In: GAGNE, R.M. (Hrsg.): Learning and individual differences. Columbus, Ohio, 2447.

FRANCIS, A., (1977): Smart Squash. Using Your Head to Win. Philadelphia.

FREUDENBRG, K. K., (1962): Grundriss der medizinischen Statistik. Stuttgart .

FRIEDRICH, E., (1984): Zur Taktik in den technisch-kompositorischen Sportarten. Leistungssport, 14 (4), 9-14.

FRITSCHE, P., (1978): Ein dynamographisches Informationssystem zur Messung der Schlagkraft beim Boxen. Leistungssport, 8 (2), 151-156.

FUHRER, U., (1984): Mehrfachhandeln in dynamischen Umfeldern. Vorschläge zu einer systematischen Erweiterung psychologisch-handlungstheoretischer Modelle. Göttingen.

GIBSON, J. J., (1979): The Ecological Approach to Visual Perception. Boston.

GOURGOULIS, V. ; AGGELOUSSIS, N. u. a., (2002): Comparative 3- Dimensional Kinematic Analysis of the Snatch Technique in Elite Male and Female Greek Weightlifters. The Journal of Strength and Conditioning Research, 16 (3), 359-366.

GÖTZ, B., (2001): Statistische Datenanalyse mit SPSS 10 für Windows, Universität Trier, Bd. 25.

GRADPOLOW, K., (1959): Handbuch des Boxens. Berlin.

GRAHAM, J., (2001): Exercise Technique: Exercise: Power Snatch. Strength and Conditioning Journal, 23 (1), 57 - 59.

GREBE, H., (1984): Die bösen Boxer. Frankenberg. 
GROSSER, M. ; HERMANN, H. u. a., (1987): Die sportliche Bewegung. München.

GROSSER, M. ; NEUMEIER, A., (1982): Techniktraining. München.

GROVE, J. R. ; HANRAHAN, S. J., (1988): Perceptions of mental training needs by Elite Field hockey players and their coaches. The Sport Psychologist, 2 (3), 222-230.

GUILFORD, J. P., (1964): Persönlichkeit. Weinheim.

GÜNTER, C. ; HEINZ, E., (1972): Grundlagen der Statistik für Psychologen, Pädagogen und Soziologen. Frankfurt.

HACKER, W., (1978): Allgemeine Arbeits- und Ingenieurpsychologie. Psychische Struktur und Regulation von Arbeitstätigkeiten. Berlin

HAGAN, R. D. ; SMITH, M. G. u. a., (1981): Marathon performance in relation to maximal aerobic power and training indices. Medicine and Science in Sports and Exercise, 21 (1), 3-7.

HARMS, V., (1998): Biomathematik, Statistik und Dokumentation. Kiel.

HARRE, D., (1986): Trainingslehre. Berlin.

HENATSCH, H. D. ; LANGER, H. H., (1983): Neurophysiologische Aspekte der Sportmotorik. In: RIEDER, H. ; BÖS, K. u. a. (Hrsg.): Motorik und Bewegungsforschung. Schorndorf, 27-55.

HEISE, T., (1998): Zur Strukturierung der Standkontrolle: Eine experimentelle Studie zur aktiven seitlichen Körperschwankung. SoWi Diss. Göttingen.

HESS, H., (1982) : Der taktische Ball. Bad Homburg.

HICKEY, K., (1980): Boxing - the amateur boxing association coaching manual. London. 
HIRSCHFELD, K., (1953): Boxen. Hildesheim.

HIRTZ, P., (1985) (Hrsg.): Koordinative Fähigkeiten im Schulsport. Berlin.

HIRTZ, P., (1994): Koordinative Fähigkeiten. In: SCHNABEL, G; HARRE, D. u. a. (Hrsg.): Trainingswissenschaft. Berlin, 137-146.

HIRTZ, P., (2000): Gleichgewichtskompetenz als Konstrukt und Konzept. In: HIRTZ, P ; HOTZ, A. u. a.(Hrsg.): Gleichgewicht, Schorndorf, 51-65.

HOBUSCH, P., (1981): Zu den Beziehungen zwischen der Antizipationsfähigkeit und den koordinativen Fähigkeiten. Theorie und Praxis der Körperkultur 30 (10), 774-780.

HOHMANN, A., (1994): Grundlagen der Trainingssteuerung im Sportspiel. Hamburg.

HOHMANN, A., (1994): Konzept der wissenschaftlichen Trainingsberatung. In: BRACK, R. ; HOHMANN, A. u. a. (Hrsg.): Trainingssteuerung, Stuttgart, 15-29.

HOHMANN, A., (1996): Zum langfristigen Aufbau des Taktiktrainings. In: STARISCHKA, S. ; CARL, K. u. a. (Hrsg.): Schwerpunktthema "Nachwuchstraining". Erlensee, 64-75.

HOHMANN, A., (1997): Wettkampfdiagnostik. In: TSCHIENE, P. ; NICKEL, H. (Hrsg.): Der sportliche Wettkampf. Münster, 144-190.

HOHMANN, A., (1999a): Anwendungs- und Grundlagenorientierung in der Trainings- und Bewegungsforschung. In: WIEMEYER, J. (Hrsg.): Forschungsmethodologische Aspekte von Motorik und Training in Sport. Hamburg. 37-54

HOHMANN, A., (1999b): Feldforschung in der Trainingswissenschaft. In: HOHMANN, A. ; WICHMANN, E. ; CARL, K. (Hrsg.): Feldforschung in der Trainingswissenschaft. Köln. 13-35. 
HOHMANN. A. ; EDELMANN-NUSSER, J., (2001): Technische Systeme bei der Trainingssteuerung. In: HUMMEL, A. ; RÜTTEN, A. (Hrsg.): Handbuch Sporttechnologie. Schorndorf, 161-173.

HOHMANN, A. ; LAMES, M. u. a., (2002): Einführung in die Trainingswissenschaft. 2. Auflage. Wiebelsheim.

HOHMANN, A. ; RÜSTEN, A., (1995): Wissenschaftliche Trainingsberatung Ein interdisziplinäres Konzept. Sportwissenschaft, 25 (2), 137-157.

HORNER, E. ; LEE, C. u. a., (1993): Creatine Kinase and neuron-specific enolase: serum markers of cell damage in the central nervous system in boxers, Cein. J. of Sport Med, 3 (3), 144-148.

HOSSNER, E. J., (1995): Module der Motorik - Bausteine des Bewegens. Schorndorf.

HOTZ, A., (1993): Lernen und Lehren - Theorie und Praxis, Magglingen, 50 (10), 18-22.

HOWALD, H., (1985): Morphologische und funktionelle Veränderungen der Muskelfaser durch Training. In: BÜHRLE, M. (Hrsg.): Grundlagen des Maximalund Schnellkrafttrainings. Schorndorf, 35-52.

IRANYI, P., (1973): Taktik und Technik beim Fechten, Leistungssport, 3 (6), 418-423.

ISRAEL, S., (1994): Die Auswirkungen eines Krafttrainings in Abhängigkeit von Lebensalter und Gesundheitszustand. In: KOMI, P.V. (Hrsg.): Kraft und Schnellkraft im Sport. Köln, 315-323.

JÄGER, G., (1994): 150 Sportarten. München. 
JOHANNPETER, K., (1979): Wettkampfbeobachtung im Boxen. In: NICKEL, H. (Hrsg.): Informationen zum Training. 18, Kampfsport I, Sportwissenschaftliche Beitrage zum Boxen, Fechten, Judo, Karate und Ringen. Berlin, 87-95.

JOHN, P. (1997): Statistisch gesehen. Grundlegende Ideen der Statistik leicht erklärt. Berlin.

JOHANSSON, G., (1978): Visual Event Perception. In: HELD, R. ; LEIBOWITZ, H. W. u. a. (Hrsg.) : Perception: Handbook of Sensory Physiology, Bd. 8. New York.

JONATH, U. ; KREMPEL, R., (1985): Konditionstraining. Training- TechnikTaktik. Reinbek.

JOST, J. ; WEISS, M. u. a., (1994): Catecholamines and chemical metabolites as physiological characteristics of performance diagnostic in punch force tests of boxers. In: LIESEN, H. ; WEISS, M. u. a. (Hrsg.): Regulations- und Repairmechanismen. 33. Deutscher Sportärztekongress Paderborn 1993. Köln, 100103.

KACURIN, A. I. ; KISESEV, V. A., (1993): Wege zur Vervollkommnung der Differenzierung der Schnelligkeit und Stärke der Schläge im Boxen. In: DUBNOVA, I. ; SVECIKOVA, N. (Hrsg.): Arbeiten der wissenschaftlichen Mitarbeiter der Staatlichen Sporthochschule. Moskau, 227-229. (Abstract auf deutsch in Spolit)

KALINOWSKI, A. G., (1985): The development of Olympic swimmers. In: BLOOM, B. S. (Hrsg.): Developing talent in Young people. New York, 139-192.

KAMINSKI, G., (1981): Überlegungen zur Funktion von Handlungstheorien in der Psychologie. In: LENK, H. (Hrsg.): Handlungstheorien interdisziplinär III, Erster Halbband. München, 93-121. 
KAMINSKI, G., (1982): What Beginner Skiers can Teach us about Actions. In: von CRANACH, M. ; HARRE, R. (Hrsg.): The Analysis of Action. Recent Theoretical and Empirical Advances. Cambridge, 99-114.

KAMINSKI, G. ; MAYER, R. u. a., (1984): Kinder und Jugendliche im Hochleistungssport. Schorndorf.

KELLER, V. S., (1976): Reflexives Handeln in der Taktik des Einzelkampfes und der Spiele. Teor. Prakt. fiz. Kult, 39 (8), 9-11. (Abstract auf deutsch in Spolit)

KIRCHGÄSSNER, H. ; BASTIAN, M., (1984): Zur Ausbildung der Handlungsschnelligkeit in den Zweikampfsportarten dargestellt an der Sportart Boxen. Theorie und Praxis der Körperkult, 33 (2), 92-96.

KIRCHGÄSSNER, H. ; FIEDLER, M. u. a., (1993): Zur Erhöhung der Wirksamkeit des technisch-taktischen Trainings. Leipziger Beiträge zur Sportwissenschaft, 34 (1), 1-15.

KIRCHGÄSSNER, H. ; WAGNER, K., (1971): Untersuchungen zur Objektivierung sportartspezifischer Schnelligkeitsleistungen im Boxen. Theorie und Praxis der Körperkult, 20 (2), 117-125.

KNAPP, B., (1977): Skill in sport. London.

KNEBEL, K. P., (1985): Funktionsgymnastik. Reinbek.

KNUTTGEN, H. G., ; KOMI, P. V., (1994): Basale Definitionen der muskulären Aktivität. In: KOMI, P.V. (Hrsg.): Kraft und Schnellkraft im Sport. Köln, 15-16.

KOCH, P., (1997): Sportartübergreifende Ausbildung spezifischer koordinativen Fähigkeiten in den Individual-Ruckschlagspielen. In: HOFFMANN, P. ; KOCH, P. (Hrsg.): Integrative Aspekte in Theorie und Praxis der Rückschlagspiele. Hamburg, 25-39. 
KOMI, P. V., ; HÄKKINEN, K., (1993): Maximalkraft und Schnellkraft. In: DIRIX, A. u. a. (Hrsg.): Olympia Buch der Sportmedizin. Köln, 157-167.

KONZAG, G., (1981): Zur Bedeutung und Diagnostik der Distribution und Konzentration der Aufmerksamkeit von Sportspielern. In: SCHELLENBERG, H.(Hrsg.): Psychologie im Sportspiel. Berlin, 36-59.

KONZAG, G. ; KONZAG, J., (1981): Kognitive Funktionen in der psychischen Regulation sportlicher Spielhandlungen und Folgerungen für den Ausbildungsprozess. In: SCHELLENBERG, H. (Hrsg.): Psychologie im Sportspiel. Berlin, 15-35.

KONZAG, J. ; KONZAG, G., (1980): Anforderungen an die kognitiven Funktionen in der psychischen Regulation sportlicher Spielhandlungen. Theorie und Praxis der Körperkultur, 29 (1), 20-31.

KÖRNDLE, H., (1985): Experimentelle Untersuchungen zur Schemageleiteten Kontrolle motorischer Handlungen. In: HAHN, E. ; SCHOCK, K. (Hrsg.): Beiträge zu Kognition und Motorik. Köln, 76-81.

KRAMPE, R.T. ; ENGBERT, R. u. a., (2000): Koordination und Synchronisation der Hände beim rhythmischen Timing. In: MÜLLER, K. ; ASCHERSLEBEN, G. (Hrsg.): Rhythmus. Ein interdisziplinäres Handbuch. Bern, 163-181.

KRAMPE, R.T. ; KLIEGL, R. u. a., (2000): The Fast and the Slow of Skilled Bimanual Rhythm Production: Parallel versus Integrated Timing. Journal of Experimental Psychology, Human Perception and Performance, 26 (3), 206-233.

KRÜGER, A., (1982a): Die Reaktionszeit des Sportlers. Ein Überblick über ausgewählte Forschungsergebnisse. Informationen zum Training. Beiheft zu Leistungssport, 31, 4-34. 
KRÜGER, A., (1982b): Reaktion und Antizipation im Basketball als Voraussetzung von Technik und Taktik, Informationen zum Training. Beiheft zu Leistungssport, 31, 34-59.

KRÜGER, A., (1994): Valentin Trichters Erben. Das Theorie-Praxis-Problem in den Leibesübungen an der Georg-August-Universität (1734 - 1987), in: SCHLOTTER, H. G., (Hrsg.): Die Geschichte der Verfassung und der Fachbereiche der Georg-August-Universität Göttingen. Göttingen, 284 - 294.

KRÜGER, A. ; NIEDLICH, D., (1985): 100 Ballspiel-Fertigkeitstests. Schorndorf.

KULIEV, O. A., (1980): Vervollkommnung der Nahkampftechnik im Boxen. Teor. Prakt. fiz. Kult, 43 (3), 8-11. (Abstract auf deutsch in Spolit)

KULIEV, O. A., (1981): Die Technik des Nahkampfes im Boxen. Teor. Prakt. fiz. Kult, 44 (9), 19-21. (Abstract auf deutsch in Spolit)

KÜRZEL, F. ; WASTL, P., (1997): Fitness Boxen. Trainingsprogramme für zu Hause. Niedernhausen.

LACKNER, K., (1995): Strafgesetzbuch mit Erläuterungen, 21- Aufl., München.

LANGHOFF, H. ; SCHOTT, M., (1983): Zum Karate Training im Breitensport Hamburger Hochschulen. Hochschulsport, 10 (9), 11-16.

LATYSENKO, V. V. ; RODIONOV, A. V., (1977): Sensomotorische Merkmale der psychischen Anspannung beim Boxen. Teor. Prakt. fiz. Kult, 40 (10),15-18. (Abstract auf deutsch in Spolit)

LAUDIN, H., (1977): Physiologie des Gedächtnisses. Heidelberg.

LEE, D.N., (1978): The Function of Vision. In: PICK, H.; SALZMANN, E., (Hrsg.): Modes of Perceiving and Processing Information. Hillsdale. 
LEE, D. N., (1980): Visuo- Motor Coordination in Space-Time. In: STELMACH, G.E. ; REQUIN, .J. (Hrsg.): Tutorials in Motor Behavior. Amsterdam, 281-295.

LEHMANN, G., (2000): Ausdauertraining in Kampfsportarten. Münster.

LEHMANN, G., (2003): Nachwuchstraining in den Zweikampfsportarten - Ergebnisse und Erfahrungen. In: SCHUMANN, K.; GARCIA, R. (Hrsg.): Die Entwicklung der Trainingssystems in den Gruppen der Sportarten (= Sport. Leistung. Persönlichkeit, Bd. 4). Schkeuditz, 35 - 44.

LEIST, K. H. ; LOIBL, J. u. a., (1983): Wahrnehmung als Grundlage von Bewegung und Bewegungslernen. In: RIEDER, H. ; BÖS, K. ; MECHLING, H. u. a. (Hrsg.): Motorik und Bewegungsforschung. Schorndorf, 260-279.

LEMME, W., (1991): Relation between the incidence of injuries and equipment. Equipment in Amateur Boxing, World Amateur Boxing Mag, 22 (5), 26-27.

LEONARD, W. M. II., (1984): A Sociological Perspective of Sport. Minneapolis.

LETZELTER, M., (1978): Trainingsgrundlagen. Hamburg.

LETZELTER, M., (1987): Training. In: EBERSPÄCHER, H. (Hrsg.) Handlexikon Sportwissenschaft. Reinbek, 483-503.

LIPPMANN, J. ; SPITZ, E. u. a., (2000): Trainingsgestaltung und Trainingsplanung im Nachwuchsbereich. Gewichtheben des Bundesverbandes Deutscher Gewichtheber e.V., o. O.

MAHONEY, M. J., (1979): Cognitive skills and athletic performance. In: KENDALE, P. C. ; HOLLON S. D. (Hrsg.): Cognitive behavioral intervention. New York, 423-443. 
MÄRKER, K., (1979): Die sportmedizinische Betreuung des Übungs-Trainingsund Wettkampfbetriebes im Boxen. Med. u. Sport, 19 (4/5/6), 115-118.

MARTIN, D., (1977): Grundlagen der Trainingslehre. Teil I, Schorndorf.

MARTIN, D., (1989): Probleme des Techniktrainings im Sport: Teil 1. Leistungssport, 19 (1), 9-13.

MARTIN, D., (1991): Merkmale einer trainingswissenschaftlichen Theorie des Techniktrainings. In: DAUGS, R. ; MECHLING, H. ; BLISCHKE, K. ; OLIVIER, N. (Hrsg.), Sportmotorisches Lernen und Techniktraining, Schorndorf.

MARTIN, D. ; CARL, K. u. a., (1991): Handbuch Trainingslehre. Schorndorf.

MARTIN, D. ; LEHNERTZ, K., (1989): Probleme des Techniktrainings im Sport: Teil 2. Leistungssport, 19 (2), 10-17.

MAXEINER, J., (1988): Konzentration und Distribution der Aufmerksamkeit im Sport. Sportwissenschaft, 18 (4), 409-420.

MAXWELL, R., (2000): Boxing 2000. New York.

MCLATCHIE, G., (1986): Verletzungen im Kampfsport. Köln.

MECHLING, H., (1984) : Bewegungswissenschaft. In: CARL, K. ; KAYSER, D. u. a. (Hrsg.): Handbuch Sport. Wissenschaftliche Grundlagen von Unterricht und Training. Band 1, Düsseldorf, 83-131.

MECHLING, H., (1988): Zur Theorie und Praxis des Techniktraining. Problemaufriss und Thesen. Leistungsport, 18 (1), 39-42.

MECHLING, H. ; CARL, K., (1992): Technik, sportliche (technique). In: RÖTHIG, P. (Leitung), BECKER, H. ; CARL, K. u. a. (Hrsg.): Sportwissenschaftliches Lexikon. Schorndorf, 504- 514. 
MEINEL, K. ; SCHNABEL, G., (1977): Bewegungslehre. Berlin.

MEINHARDT, B., (1996): Boxen in Deutschland. Hamburg.

MISCHEL, W., (1968): Personality and assessment. New York.

MONSAAS, J. A., (1985): Learning to be a world-class tennis player. In: BLOOM, B.S. (Hrsg.) Developing talent in young people. New York, 211-269.

MOORE, M., (1980): The challenge of boxing. Bringing safety into the ring. Physician and Sports Med, 8 (11), 101-105.

MÜLLER, D., (1991): Letzter Kampf in Olympia. Nowojski.

MÜLLER-DECK, H., (2003): Zur Entwicklung des Trainingssystems in den Zweikampfsportarten im Leistungssport der DDR. In: SCHUMANN, K.; GARCIA, R. (Hrsg.): Die Entwicklung der Trainingssystems in den Gruppen der Sportarten (= Sport. Leistung. Persönlichkeit, Bd. 4). Schkeuditz, 7-24.

NEUMAIER, A., (1999): Koordinatives Anforderungsprofil und Koordinationstraining. Köln.

NEUMAIER, A. ; MECHLING, H., (1994): Taugt das Konzept der ,Koordinativen Fähigkeiten” als Grundlage für sportartspezifisches Koordinationstraining ? In: BLASER, P. ; WITTE, K. u. a. (Hrsg.): Steuer- und Regelungsvorgänge der menschlichen Motorik. St. Augustin, 207-212.

NEUMAIER, A. ; MECHLING, H., (1995): Allgemeines oder sportartspezifische Koordinationstraining ? Ein Strukturierungsvorschlag zur Analyse und zum Training spezieller Koordinative Leistungsvoraassetzungen. Leistungssport, 25 (5), 14-18. 
NEUMANN, J ; MORGENSTERN, O., (1974): Theory of Games and Economic Behavior. Princeton.

NEUMANN, O., (1992): Theorien der Aufmerksamkeit: von Metaphern zu Mechanismen. Psychologische Rundschau, 43 (4), 83-101.

NITSCH, J. R., (1982): Handlungspsychologische Ansätze im Sport. In: THOMAS, A. (Hrsg.): Sportpsychologie. Ein Handbuch in Schlüsselbegriffen. Weinheim, 26-41.

NEWTON, H., (2002): Explosive Lifting for Sports. Boost power with the Snatch, Clean, Jerk, Squat, and other Dynamic lifts. Champaign, IL.

NITSCH, J. R. ; NEUMAIER, A., (1997): Interdisziplinäres Grundverständnis von Training und Techniktraining. In: NITSCH, J. R. ; NEUMAIER, A. (Hrsg.):Techniktraining. Schorndorf, 109-123.

NOGUCHI, K. ; HAISHI, K. u. a., (1994): Die Illusion von Geschwindigkeit bei der Bewegungswahrnehmung. Percept . mot. Skills , 78 (1), 112-114.

OATES, J.C., (1988): Über Boxen. Zürich.

OBERSTE, W., (1979): Sensomotorische Leistungen beim Tiefstart und Staffellauf. Schorndorf.

OGURENKO, E., (1972): Der Nahkampf im Boxen - die Taktik des Nahkampfs. Frankfurt am Main.

OKONEK, CH., (1987): Täuschungshandlungen im Sport. Theoretische Analyse von Täuschungssituationen und eine Fallstudie zum Fintierverhalten im Basketball. Sowi Dissertation, Göttingen.

ORLICK, T. ; PARTINGTON, J., (1988): Mental links of excellence. The Sport Psychologist, 2 (2), 105-230. 
PAPAGEORGIOU, A. ; SPRITZLEY, W., (1986): Täuschungsaufstellungen. In: CHRISTMANN, E. ; LETZELTER, H. (Hrsg.): Spielanalysen und Trainingsmaßnahmen im Volleyball. 11.Symposium des DVV 1985. Ahrensburg, 178203.

PAPALAS, A. J., (1984): The development of boxing. In: Ancient Word, 9 (3/4), 67-76.

PÖHLMANN, R. ; ENDERS, B., (1983) : Sportmotorische Handlungsregulation als Informationsverarbeitender Prozess - eine experimentelle Nachprüfung der Einkanalhypothese. Theorie und Praxis der Körperkultur, 32 (8), 587-591.

POLIAKOFF, M. B., (1987): Combat sports in the ancient word. New Haven.

POLIAKOFF ; M. B., (1989): Kampfsport in der Antike. Das Spiel um Leben und Tod. München.

PORSUGJAN, E. V. ; FARFEL, V. S., (1978): Dauer der Schlagausführung beim Boxen und ihre zeitliche Verkürzung. Teor. Prakt. fiz. Kult, 41 (10), 15-18. (Abstract auf deutsch in Spolit)

POULTON, E. C., (1950): Anticipation in open and closed sensorimotor skills. Cambridge.

RAAB, M., (1999): Taktiklernen im Sportspiel. In: KRUG ,J. ; HARTMANN, C. (Hrsg.): Praxisorientierte Bewegungslehre als angewandte Sportmotorik. Sankt Augustin, 93-99.

RÄSCH, W., (1973): Handbuch zu den Wettkampfbestimmungen Boxen. 2Aufl., Berlin.

REDO, R. ; TILEWSKI, G., (1990): Ein Computergestütztes Punktwertungssystem im Boxen. Sport wyczynowy, 28 (1/2), 34- 37. (Abstract auf deutsch in Spolit) 
REED, S. K., (1978): Schemes and Theorys of Pattern Recognition. In: CARTERETTE, E. C. ; FREEDMANN, M. P. (Hrsg.): Handbook of Perception. Vol. IX. Perceptual Processinq, New York,137-162.

REICH, T., (1964): Idee und Praxis der medizinischen Statistik. Bern.

RENZLAND, J. ; EBERSPÄCHER, H., (1988): Regeneration im Sport. Köln.

RIECKERT, H., (1993): Krafttraining im Breitensport. Deutsche Zeitschrift für Sportmedizin, 44 (7), 312- 322.

RITZDORF, W., (1982): Visuelle Wahrnehmung und Antizipation. Eine theoretische und experimentelle Studie zum Entscheidungs- und Blickverhalten beim Betrachten von Tennisgrundschlägen. Schorndorf.

ROCK, J., (1985): Wahrnehmung. Vom visuellen Reiz zum Sehen und Erkennen. Heidelberg, 187-190.

ROHLAND, U., (2000): Statistik Erläuterung grundlegender Begriffe und Verfahren. Aachen.

ROMANOV, V. M. ; BAJKOV, S, S., (1978): Taktik- und Techniktraining im Boxen. Teor. Prakt. fiz. Kult, 41 (4), 15-18. (Abstract auf deutsch in Spolit)

ROSS, R. ; CASSON, I. u. a., (1987): Boxverletzungen: Neurologische, radiologische und neuropsychologische Auswertung. Clin. Sport Med, 6 (1), 41-51. (Abstract auf deutsch in Spolit)

RÖTHIG, P., (1992) (Hrsg.): Sportwissenschaftliches Lexikon. 6- Aufl., Schorndorf.

ROTH. K., (1996): Techniktraining im Spitzensport. Köln. 
RUNESON, S. ; FRYKHOLM, G., (1981): Visual Perception of Lifted Weight. Journal of Experimental Psychology: Human Perception and Performance, 7 (2), 733-740.

RUSHALL, B. S. ; HALL, M. u. a., (1988): Three types of thought content instruction on skiing performance. The Sport Psychologist, 2 (4), 283-297.

RÜSSEL, A., (1976) : Psychomotorik. Darmstadt:.

SACK, H. G., (1980): Zur Psychologie des jugendlichen Leistungssportlers. Schorndorf.

SANDERS, A. F., (1979): Some Remarks on Mental Load. In: MORAY, N. (Hrsg.): Mental Workload. New York, 41-77.

SCHBÖ, W ; PERLETH, CH. u. a., (1991): SPSS Kompakt für die Versionen 3 und 4 . Stuttgart.

SCHIEFLER, B., (1982): Zur Antizipation im Tischtennis und den Möglichkeiten, sie zu beeinflussen. DTS Lehre und Praxis, 10 (4), 52-53.

SCHLIMPER, L., (1989): Objektivierung kognitiver Leistungsvoraussetzungen und Interpretation experimenteller Ergebnisse in der Sportart Boxen. Wiss. Z. d. DHfK , 30 (1), 75-88. (Abstract auf deutsch in Spolit)

SCHMIDT, D. ; BRAUN, P. u. a, (1987): Gerätturnen- Anleitung für den Übungsleiter. Berlin.

SCHMIDT, R. A., (1975): A Schema Theory of Discrete Motor-Skill Learning. Psychological Review, 8 (2), 225-260. 
SCHMIDT, R. A., (1976): The Schema as a Solution to Some Persistent Problems in Motor Learning Theory. In: STELMACH, G. E.(Hrsg.): Motor Control: Issues and Trends. New York, 41-65.

SCHMIDT, R. A., (1980): Issues in Motor Programming. Research Quarterly for Exercise and Sport, 51 (1), 122-140.

SCHMIDT, R. A., (1982): Motor Control and Learning. A Behavioral Emphasis. Champaign, IL.

SCHMIDT, R. A. ; GORDON, G. W., (1977): Errors in Motor Responding, Rapid Corrections, and False Anticipations. Journal of Motor Behavior, 9 (2), 101-111.

SCHNABEL, G. ; HARRE, D. u. a., (1994) (Hrsg.): Trainingswissenschaft. Leistung- Training- Wettkampf. Berlin.

SCHENKER, Z. O., (1961): Säbelfechten. Budapest.

SCHNEIDER, W., (1988): Zur Rolle des Wissen bei kognitiven Höchstleistungen. Psychologie in Erziehung und Unterricht, 35 (4), 161-172.

SCHOCK, K. K., (1984) : Taktische Fertigkeiten und ihre methodische Vermittlung. Leistungssport, 14 (1), 5-12.

SCHÖFFLER, H., (1986): England, das Land des Sports. Eine Kultursoziologische Erklärung. Münster.

SCHÖNBORN, R., (1990): Gedanken zur Taktik im Tennis. Tennis-Sport, 1 (3), 2-7.

SCHÖNKE, A. ; SCHRÖDER, H., (1991): Strafgesetzbuch Kommentar, 24Aufl., München. 
SCHULZ, A., (1984): Zur Entwicklung von Technik und Taktik im Volleyball. In: CHRISTMANN, E. (Hrsg.): Volleyball trainieren. Ahrensburg, 257-269.

SCHWIRTZ, A., (1994): Bewegungstechnik und muskuläre Koordination beim Skilanglauf. Köln.

SEKULES, K., (2000): The boxer's heart. How I fell in love with the ring. New York.

SIEGEL, S. ; CASTELLAN, J., (1988): Non parametric statistics for behavioral sciences. New York.

SINZ, R., (1981): Lernen und Gedächtnis. Stuttgart.

SMITH, B., (1983): Recreational Drugs in Sports. The Physician and Sports Medicine, 11 (9), 75-82.

SONNENBERG, H., (1978): Boxen. Fechten mit der Faust. Berlin.

SONNENBERG, H., (1989): Boxen. Fechten mit der Faust. Berlin.

SONNENBERG, H., (1993): Boxen. Fechten mit der Faust. Berlin.

STAUFFER, R., (1993): Boxerrunden. München.

STELMACH, G. E. ; REQUIN, J., (1992) : Tutorials in motor behavior. Amsterdam.

STEMMLER, R. ; BECHER, H. u. a., (1980) : Statistische Methoden im Sport. Berlin.

STEWART, W. F. ; GARDEN, B. u. a., (1994): A Prospective study of centralnervous System function in amateur boxers in the United States. World Amateur Boxing Mag, 13 (9), 3-26. 
THIE, P. R., (1979): An Introduction to Linear Programming and Game Theory. New York, 284-290.

TOPYSCHJOW, O. P. ; DSHEROJAN, G. O., (1979): Einige Fragen zur Schlagtechnik im Boxen. In: NICKEL, H. (Hrsg.): Informationen zum Training, 19, 32-41.

TORAN, G., (1995): Strategie und Taktik im Fechten. Scuola dello Sport (sds) Riv. di cult. Sport, 14 (32), 56-61. (Abstract auf deutsch in Spolit)

TRAMPISCH, W., (1997) (Hrsg.): Medizinische Statistik. Heidelberg.

TRUMPOLD, J., (1980): Die technisch-taktische Grundausbildung in der Sportart Boxen. Theorie und Praxis Leistungssport, 18 (5),140-147.

TURVEY, M. T. ; TREFFNER, P. J., (1995): Handedness and the asymmetric dynamics of bimanual rhythmic coordination. J. of exper. Psychol, 21 (2), 318333.

TYSLER, D. ; TYSLER, G., (1995): Taktik des Fechtkampfes. Teor. i Prakt. fiz. Kul't, 21 (7), 28-29. (Abstract auf deutsch in Spolit)

ULLRICH, K ; GOLLHOFER, A., (1994): Physiologische Aspekte und Effektivität unterschiedliche Dehnmethoden. Deutsche Zeitschrift für Sportmedizin, 45 (9), 336-345.

VALENTINO, B. ; ESPOSITO, L. C. u. a., (1990): Electromyographic activity of a muscular group in movements specific to boxing. J. of Sports Med. phys. Fitness, 30 (2), 160-162.

VEALEY, R. S., (1988): Future directions in psychological skills training.The Sport Psychologist, 2 (4), 318-336. 
VOGT, U., (1991): Basketball für Kinder und Jugendliche in Schule und Verein Spiel- und Übungsformen zur Vermittlung von Taktik. Sportpraxis, 32 (2), 50-52.

VORONIN, E. V., (1983): Der Einfluss psychomotorischer Schnelligkeitsmerkmale auf die Taktik im Tischtennis. Teor. Prakt. fiz. Kult, 46 (10), 52-54. (Abstract auf deutsch in Spolit)

WARREN, W. H. ; SHAW, R. E., (1985) (Hrsg.): Persistence and Change, Hillsdale.

WEILER, I., (1995): Boxen. Quellendokumentation zu Gymnastik und Agonistik im Altertum. Köln.

WEINMANN, W., (1995): Das Kampfsportlexikon, Berlin.

WEISE, K., (1995): Henry Maske - auf eigene Faust. Berlin.

WIEMANN, K., (1991): Beeinflussung muskulärer Parameter durch ein zehnwöchiges Dehntraining. Sportwissenschaft, 21 (3), 292-306.

WIEMANN, K. ; KLEE, A., (2000): Dehnen und Stretching in der Aufwärmphase vor Höchstleistungen. Leistungsport, 30 (4), 5-9.

WILLIAMS, L. R. T. ; SULLIVAN, S. J., (1978) : Effects of Movement Speed on the Refractorines of an Extended Movemen to Reversal. Journal of Human Movement Studies 4 (1), 70-84.

WILLIMCZIK, K., (1978): Die motorische Entwicklung des Kindes. In: ERWIN, H; GUENTER, K. u. a. (Hrsg.): Kind und Bewegung. Kinderturnen kritisch betrachtet, Schorndorf, 21, 79-90.

WILLIMCZIK, K., (1991): Techniktraining - Eine Symbiose für Lern- und Adaptationsprozesse? In: DAUGS, R. u. a. (Hrsg.): Sportmotorisches Lernen und Techniktraining, Band 1. Schorndorf, 132-146 
WILLIMCZIK, K., (1992): Statistik im Sport. Grundlagen, Verfahren, Anwendungen. Frankfurt am Main.

WILLIMCZIK, K., (1993): Statistik im Sport. Hamburg.

WILLIMCZIK, K. ; ROTH, K., (1983): Bewegungslehre. Reinbek bei Hamburg.

WRISBERG, C. A. ; RAGSDALE, M. R., (1979): Cognitive demand and practice level. Factors in the mental rehearsal of motor skills. J. Human Movement Stud, 5 (4), 201-208. 
http://www.andreas-albert.de/Boxen.html (entnommen am: 16.08.2003, 20:12 Uhr)

http://www.mixed-martial-arts.de/boxen.html (entnommen am:13.04.2001, 16:05 Uhr)

http://users.skynet.be/Boxring-Eupen/Plaire/html/body boxgeschichte.html (entnommen am:15. 08. 2003, 21:17 Uhr)

http://www.sportgericht.de/Sportarten/Boxen/BoxenHistorie.htm (entnommen am: 17. 09. 2003, 21:36 Uhr)

http://www.boxen-sport.de/Archiv/news archiv5.htm (entnommen am:13.09.2003, 22:19 Uhr)

http://www.kontaktrunde.de/boxen/gewichtsklassen.html_ (entnommen am:10.10. 2003, 16:09 Uhr)

http://www.psv-georgsmarienhuette.de/box geschichte.html (entnommen am:16. 09.2001, 21:07 Uhr)

http://www.aiba.net/zak/olympicresults.html_(entnommen am: 21.07.2001, 22:19 Uhr) 


\title{
13. Anhang
}

\section{E-Mail von Prof. Dr. Arnd Krüger an die Abt. Images and Sound des loc}

Tel. $(+49) 551-395651$

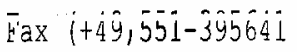

Internet: http://www.sport.uni-goettingen.de

e-mail: akruegel@gwdg.de

\author{
Subject: Olympic Games Sydney 2000 / Boxing - Your E-mail dated 19th March 2002 to \\ Mr. Didier Blanchard \\ Date: Wed, 20 Mar 2002 09:29:36 +0100 \\ From: Monique Perusset <monique.perusset@o,olympic.org> \\ Organization: olympic.org \\ T0: akruegel@gwdg.de
}

Dear Mr. Krueger,

We refer to the above-mentioned E-mail for which we thank you very much.

For your information, the Olympic Museum archives contain more than

17'500 hours of animated images, mainly world feed. Therefore, in order

to obtain video tapes, your student should simply send us a specific

list of the events he is looking for together with the names of the

competitors, the format required for the video tapes and explain us how he is going to use the footage. We shall then proceed with research in our archives and once this has been accomplished, we will be better able to provide him with the details of the material available and an estimate of the expenses involved. In order for us to be able to do that, your student should give us his complete coordinates (full address, phone and/of fax number is any available).

He can also make a selection himself at the Olympic Museum by appointment.

We are at your disposal for any further information you may need and look forward to hearing from you.

Yours sincerely,

Monique Perusset

Images \& Sound Department

Olympic Museum, Lausanne, Switzerland

Tel. no. ++41.21 .621 .66 .37$

Fax no. ++41.21 .621 .65 .12$

E-mail address : monique.perusset@olympic.org 


\section{Zeitplan Boxen: Sydney 2000}

\begin{tabular}{|c|c|c|c|}
\hline Datum & Zeit & Veranstaltungen & Spielort \\
\hline \multirow[t]{2}{*}{ Sa 16. Sept. } & $\begin{array}{l}\text { 03:00-06:00 } \\
\text { Uhr }\end{array}$ & $\begin{array}{l}\text { Bantamgewicht (54kg) Spieltag 1, Weltergewicht } \\
(67 \mathrm{~kg}) \text { Spieltag } 1\end{array}$ & $\begin{array}{l}\text { Sydney Exhibition } \\
\text { Centre }\end{array}$ \\
\hline & $\begin{array}{l}\text { 09:30-12:30 } \\
\text { Uhr }\end{array}$ & $\begin{array}{l}\text { Bantamgewicht (54kg) Spieltag 1, Weltergewicht } \\
(67 \mathrm{~kg}) \text { Spieltag } 1\end{array}$ & $\begin{array}{l}\text { Sydney Exhibition } \\
\text { Centre }\end{array}$ \\
\hline \multirow[t]{2}{*}{ So 17. Sept. } & $\begin{array}{l}\text { 03:00-06:00 } \\
\text { Uhr }\end{array}$ & $\begin{array}{l}\text { Halbfliegengewicht (48kg) Spieltag 1, Leichtgewicht } \\
(60 \mathrm{~kg}) \text { Spieltag } 1\end{array}$ & $\begin{array}{l}\text { Sydney Exhibition } \\
\text { Centre }\end{array}$ \\
\hline & $\begin{array}{l}\text { 09:30-12:30 } \\
\text { Uhr }\end{array}$ & $\begin{array}{l}\text { Halbfliegengewicht (48kg) Spieltag 1, Leichtge- } \\
\text { wicht }(60 \mathrm{~kg}) \text { Spieltag } 1\end{array}$ & $\begin{array}{l}\text { Sydney Exhibition } \\
\text { Centre }\end{array}$ \\
\hline \multirow[t]{2}{*}{$\begin{array}{l}\text { Mo } 18 . \\
\text { Sept. }\end{array}$} & $\begin{array}{l}\text { 03:00-06:00 } \\
\text { Uhr }\end{array}$ & $\begin{array}{l}\text { Mittelgewicht (75kg) Spieltag 1, Federgewicht }(57 \mathrm{~kg}) \\
\text { Spieltag } 1\end{array}$ & $\begin{array}{l}\text { Sydney Exhibition } \\
\text { Centre }\end{array}$ \\
\hline & $\begin{array}{l}09: 30-12: 30 \\
\text { Uhr }\end{array}$ & $\begin{array}{l}\text { Mittelgewicht (75kg) Spieltag 1, Federgewicht(57kg) } \\
\text { Spieltag } 1\end{array}$ & $\begin{array}{l}\text { Sydney Exhibition } \\
\text { Centre }\end{array}$ \\
\hline \multirow[t]{2}{*}{ Di 19. Sept. } & $\begin{array}{l}\text { 03:00-06:00 } \\
\text { Uhr }\end{array}$ & $\begin{array}{l}\text { Halbmittelgewicht }(71 \mathrm{~kg}) \text { Spieltag 1, Fliegenge- } \\
\text { wicht }(51 \mathrm{~kg}) \text { Spieltag } 1\end{array}$ & $\begin{array}{l}\text { Sydney Exhibition } \\
\text { Centre }\end{array}$ \\
\hline & $\begin{array}{l}\text { 09:30-12:30 } \\
\text { Uhr }\end{array}$ & $\begin{array}{l}\text { Halbmittelgewicht }(71 \mathrm{~kg}) \text { Spieltag 1, Fliegengewicht } \\
(51 \mathrm{~kg}) \text { Spieltag } 1\end{array}$ & $\begin{array}{l}\text { Sydney Exhibition } \\
\text { Centre }\end{array}$ \\
\hline \multirow[t]{2}{*}{ Mi 20. Sept. } & $\begin{array}{l}\text { 03:00-06:00 } \\
\text { Uhr }\end{array}$ & $\begin{array}{l}\text { Halbweltergewicht (63.5kg) Spieltag 1, Halbschwer- } \\
\text { gewicht }(81 \mathrm{~kg}) \text { Spieltag } 1\end{array}$ & $\begin{array}{l}\text { Sydney Exhibition } \\
\text { Centre }\end{array}$ \\
\hline & $\begin{array}{l}\text { 09:30-12:30 } \\
\text { Uhr }\end{array}$ & $\begin{array}{l}\text { Halbweltergewicht }(63.5 \mathrm{~kg}) \text { Spieltag 1, Halbschwer- } \\
\text { gewicht }(81 \mathrm{~kg}) \text { Spieltag } 1\end{array}$ & $\begin{array}{l}\text { Sydney Exhibition } \\
\text { Centre }\end{array}$ \\
\hline \multirow[t]{2}{*}{ Do 21. Sept. } & $\begin{array}{l}\text { 03:00-06:00 } \\
\text { Uhr }\end{array}$ & $\begin{array}{l}\text { Bantamgewicht (54kg) Spieltag 2, Weltergewicht } \\
\text { (67kg) Spieltag 2, Schwergewicht( 91kg) Spieltag } 1\end{array}$ & $\begin{array}{l}\text { Sydney Exhibition } \\
\text { Centre }\end{array}$ \\
\hline & $09: 30-12: 30$ & $\begin{array}{l}\text { Bantamgewicht (54kg) Spieltag 2, Weltergewicht } \\
\text { (67kg) Spieltag 2, Schwergewicht (91kg) Spieltag } 1\end{array}$ & $\begin{array}{l}\text { Sydney Exhibition } \\
\text { Centre }\end{array}$ \\
\hline \multirow[t]{2}{*}{ Fr 22. Sept. } & $\begin{array}{l}\text { 03:00-06:00 } \\
\text { Uhr }\end{array}$ & $\begin{array}{l}\text { Halbfliegengewicht (48kg) Spieltag 2, Leichtgewicht } \\
(60 \mathrm{~kg}) \text { Spieltag 2, Mittelgewicht }(75 \mathrm{~kg}) \text { Spieltag } 2\end{array}$ & $\begin{array}{l}\text { Sydney Exhibition } \\
\text { Centre }\end{array}$ \\
\hline & $\begin{array}{l}09: 30-12: 30 \\
\text { Uhr }\end{array}$ & $\begin{array}{l}\text { Halbfliegengewicht (48kg) Spieltag 2, Leichtgewicht } \\
(60 \mathrm{~kg}) \text { Spieltag 2, Mittelgewicht }(75 \mathrm{~kg}) \text { Spieltag } 2\end{array}$ & $\begin{array}{l}\text { Sydney Exhibition } \\
\text { Centre }\end{array}$ \\
\hline \multirow[t]{2}{*}{ Sa 23. Sept. } & $\begin{array}{l}\text { 03:00-06:00 } \\
\text { Uhr }\end{array}$ & $\begin{array}{l}\text { Federgewicht }(57 \mathrm{~kg}) \text { Spieltag 2, Halbmittelgewicht } \\
(71 \mathrm{~kg}) \text { Spieltag 2, Superschwergewicht }(+91 \mathrm{~kg}) \\
\text { Spieltag } 1\end{array}$ & $\begin{array}{l}\text { Sydney Exhibition } \\
\text { Centre }\end{array}$ \\
\hline & $\begin{array}{l}\text { 09:30-12:30 } \\
\text { Uhr }\end{array}$ & $\begin{array}{l}\text { Federgewicht }(57 \mathrm{~kg}) \text { Spieltag 2, Halb Mittelgewicht } \\
(71 \mathrm{~kg}) \text { Spieltag } 2 \text {, Superschwergewicht }(+91 \mathrm{~kg}) \\
\text { Spieltag } 1\end{array}$ & $\begin{array}{l}\text { Sydney Exhibition } \\
\text { Centre }\end{array}$ \\
\hline \multirow[t]{2}{*}{ So 24. Sept. } & $\begin{array}{l}\text { 03:00-06:00 } \\
\text { Uhr }\end{array}$ & $\begin{array}{l}\text { Fliegengewicht }(51 \mathrm{~kg}) \text { Spieltag 2, Halbweltergewicht } \\
\text { (63.5kg) Spieltag 2, Halbschwergewicht }(81 \mathrm{~kg}) \\
\text { Spieltag } 2\end{array}$ & $\begin{array}{l}\text { Sydney Exhibition } \\
\text { Centre }\end{array}$ \\
\hline & $\begin{array}{l}\text { 09:30-12:30 } \\
\text { Uhr }\end{array}$ & $\begin{array}{l}\text { Fliegengewicht (51kg) Spieltag 2, Halbweltergewicht } \\
(63.5 \mathrm{~kg}) \text { Spieltag 2, Halbschwergewicht }(81 \mathrm{~kg}) \\
\text { Spieltag } 2\end{array}$ & $\begin{array}{l}\text { Sydney Exhibition } \\
\text { Centre }\end{array}$ \\
\hline \multirow[t]{2}{*}{ Di 26. Sept. } & $\begin{array}{l}\text { 03:00-06:00 } \\
\text { Uhr }\end{array}$ & $\begin{array}{l}\text { Viertelfinale: Halbfliegengewicht (48kg), Bantam- } \\
\text { gewicht (54kg), Leichtgewicht }(60 \mathrm{~kg}), \text { Weltergewicht } \\
(67 \mathrm{~kg}), \text { Mittelgewicht }(75 \mathrm{~kg}), \text { Schwergewicht }(91 \mathrm{~kg})\end{array}$ & $\begin{array}{l}\text { Sydney Exhibition } \\
\text { Centre }\end{array}$ \\
\hline & $\begin{array}{l}\text { 09:30-12:30 } \\
\text { Uhr }\end{array}$ & $\begin{array}{l}\text { Viertelfinale: Halbfliegengewicht (48kg), Bantam- } \\
\text { gewicht (54kg), Leichtgewicht }(60 \mathrm{~kg}) \text {, Weltergewicht } \\
(67 \mathrm{~kg}), \text { Mittelgewicht }(75 \mathrm{~kg}), \text { Schwergewicht }(91 \mathrm{~kg})\end{array}$ & $\begin{array}{l}\text { Sydney Exhibition } \\
\text { Centre }\end{array}$ \\
\hline \multirow[t]{2}{*}{ Mi 27. Sept. } & $\begin{array}{l}\text { 03:00-06:00 } \\
\text { Uhr }\end{array}$ & $\begin{array}{l}\text { Viertelfinale: Fliegengewicht }(51 \mathrm{~kg}) \text {, Federgewicht } \\
(57 \mathrm{~kg}) \text {, Halbweltergewicht }(63.5 \mathrm{~kg}) \text {, Halbmittelge- } \\
\text { wicht }(71 \mathrm{~kg}) \text {, Halbschwergewicht }(81 \mathrm{~kg}) \text {, Super- } \\
\text { schwergewicht }(+91 \mathrm{~kg})\end{array}$ & $\begin{array}{l}\text { Sydney Exhibition } \\
\text { Centre }\end{array}$ \\
\hline & $\begin{array}{l}\text { 09:30-12:30 } \\
\text { Uhr }\end{array}$ & $\begin{array}{l}\text { Viertelfinale: Fliegengewicht }(51 \mathrm{~kg}) \text {, Federgewicht } \\
(57 \mathrm{~kg}), \text { Halbweltergewicht }(63.5 \mathrm{~kg}) \text {, Halbmittelge- } \\
\text { wicht }(71 \mathrm{~kg}) \text {, Halbschwergewicht }(81 \mathrm{~kg}) \text {, Super- } \\
\text { schwergewicht }(+91 \mathrm{~kg})\end{array}$ & $\begin{array}{l}\text { Sydney Exhibition } \\
\text { Centre }\end{array}$ \\
\hline Do 28. Sept. & $\begin{array}{l}\text { 09:30-12:30 } \\
\text { Uhr }\end{array}$ & $\begin{array}{l}\text { Halbfinale: Halbfliegengewicht }(48 \mathrm{~kg}) \text {, Bantamge- } \\
\text { wicht }(54 \mathrm{~kg}) \text {, Leichtgewicht }(60 \mathrm{~kg}) \text {, Weltergewicht } \\
(67 \mathrm{~kg}) \text {, Mittelgewicht }(75 \mathrm{~kg}), \text { Schwergewicht }(91 \mathrm{~kg})\end{array}$ & $\begin{array}{l}\text { Sydney Exhibition } \\
\text { Centre }\end{array}$ \\
\hline Fr 29. Sept. & $\begin{array}{l}\text { 09:30-12:30 } \\
\text { Uhr }\end{array}$ & $\begin{array}{l}\text { Halbfinale: Fliegengewicht }(51 \mathrm{~kg}) \text {, Federgewicht } \\
(57 \mathrm{~kg}), \text { Halbweltergewicht }(63.5 \mathrm{~kg}) \text {, Halbmittelge- } \\
\text { wicht }(71 \mathrm{~kg}) \text {, Halbschwergewicht }(81 \mathrm{~kg}) \text {, Super- } \\
\text { schwergewicht }(+91 \mathrm{~kg})\end{array}$ & $\begin{array}{l}\text { Sydney Exhibition } \\
\text { Centre }\end{array}$ \\
\hline Sa 30. Sept. & $\begin{array}{l}\text { 03:00-05:05 } \\
\text { Uhr }\end{array}$ & $\begin{array}{l}\text { Finale: Halbfliegengewicht(48kg), Bantamgewicht } \\
\text { (54kg), Leichtgewicht }(60 \mathrm{~kg}) \text {, Weltergewicht }(67 \mathrm{~kg}) \text {, } \\
\text { Mittelgewicht }(75 \mathrm{~kg}) \text {, Schwergewicht }(91 \mathrm{~kg})\end{array}$ & $\begin{array}{l}\text { Sydney Exhibition } \\
\text { Centre }\end{array}$ \\
\hline So 1. Okt. & $\begin{array}{l}\text { 03:00-05:05 } \\
\text { Uhr }\end{array}$ & $\begin{array}{l}\text { Finale: Fliegengewicht }(51 \mathrm{~kg}) \text {, Federgewicht } \\
(57 \mathrm{~kg}) \text {, Halbweltergewicht }(63.5 \mathrm{~kg}) \text {, Halbmittel- } \\
\text { gewicht }(71 \mathrm{~kg}) \text {, Halbschwergewicht }(81 \mathrm{~kg}) \text {, Su- } \\
\text { perschwergewicht }(+91 \mathrm{~kg})\end{array}$ & $\begin{array}{l}\text { Sydney Exhibition } \\
\text { Centre }\end{array}$ \\
\hline
\end{tabular}




\section{Verteilung der Boxer nach Kategorien und Ländern Sydney 2000}

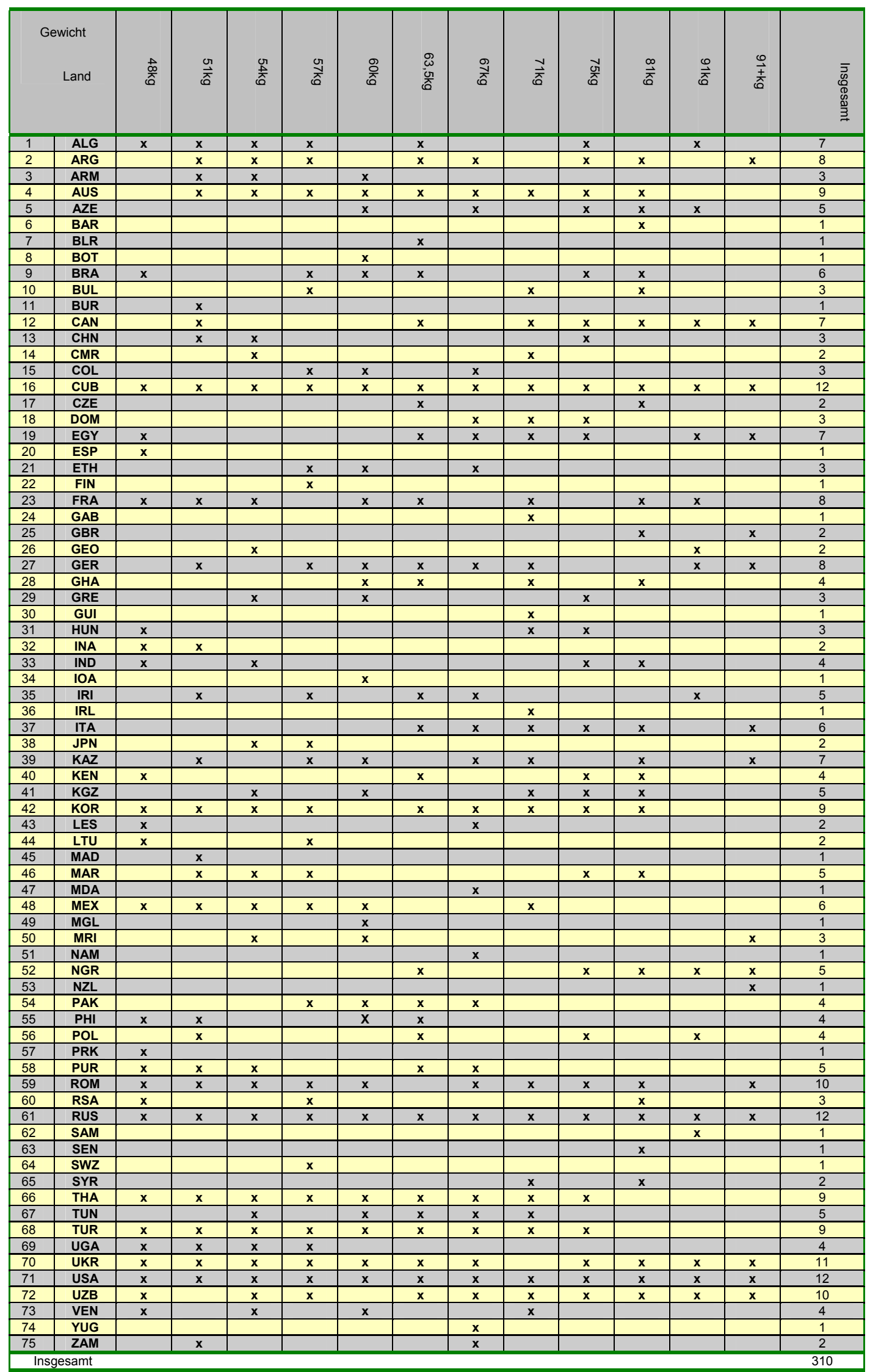




\section{Verteilung der Boxer nach Kategorien und Ländern im Halbfinale}

\begin{tabular}{|c|c|c|c|c|c|c|c|c|c|c|c|c|c|c|}
\hline \multicolumn{2}{|c|}{ Gewicht } & \multirow{3}{*}{$\underset{\hat{\sigma}}{\stackrel{\infty}{\hat{\theta}}}$} & \multirow{3}{*}{ 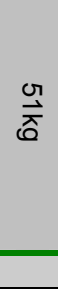 } & \multirow{3}{*}{$\begin{array}{l}\text { Ð } \\
\stackrel{\star}{\hat{\theta}}\end{array}$} & \multirow{3}{*}{ త্ત } & \multirow{3}{*}{ ᄋ } & \multirow{3}{*}{ 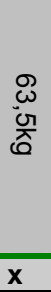 } & \multirow{3}{*}{$\underset{\text { ลี่ }}{\stackrel{D}{0}}$} & \multirow{3}{*}{$\stackrel{\vec{\lambda}}{\overrightarrow{\hat{\theta}}}$} & \multirow{3}{*}{ ัํํ } & \multirow{3}{*}{$\stackrel{\infty}{\overrightarrow{\hat{\sigma}}}$} & \multirow{3}{*}{$\stackrel{\bullet}{\stackrel{\bullet}{\hat{~}}}$} & \multirow{3}{*}{$\frac{\stackrel{\bullet}{\vec{t}}}{\stackrel{+}{\hat{\theta}}}$} & \multirow{3}{*}{ 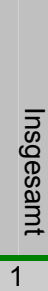 } \\
\hline & Land & & & & & & & & & & & & & \\
\hline 1 & ALG & & & & & & & & & & & & & \\
\hline 2 & AZE & & & & & & & & & $\mathbf{x}$ & & & & 1 \\
\hline 3 & CSE & & & & & & & & & & $\mathbf{x}$ & & & 1 \\
\hline 4 & CUB & $\mathbf{x}$ & & $x$ & & $\mathbf{x}$ & $\mathbf{x}$ & & & $\mathbf{x}$ & & $\mathbf{x}$ & & 6 \\
\hline 5 & ESR & $x$ & & & & & & & & & & & & 1 \\
\hline 6 & FRA & $x$ & $x$ & & & & & & & & & & & 2 \\
\hline 7 & GBR & & & & & & & & & & & & $x$ & 1 \\
\hline 8 & GEO & & & & & & & & & & & $x$ & & 1 \\
\hline 9 & GER & & & & & & & & & & & $x$ & & 1 \\
\hline 10 & HUN & & & & & & & & & $x$ & & & & 1 \\
\hline 11 & ITA & & & & & & & & & & & & $x$ & 1 \\
\hline 12 & KAZ & & $x$ & & $x$ & & & & $\mathbf{x}$ & & & & $\mathbf{x}$ & 4 \\
\hline 13 & MAR & & & & $x$ & & & & & & & & & 1 \\
\hline 14 & MDA & & & & & & & $\mathbf{x}$ & & & & & & 1 \\
\hline 15 & MEX & & & & & $x$ & & & & & & & & 1 \\
\hline 16 & PRK & $\mathbf{x}$ & & & & & & & & & & & & 1 \\
\hline 17 & ROM & & & & & & & $x$ & $\mathbf{x}$ & & & & & 2 \\
\hline 18 & RUS & & & $x$ & $\mathbf{x}$ & $\mathbf{x}$ & & $\mathbf{x}$ & & $\mathbf{x}$ & $\mathbf{x}$ & $\mathbf{x}$ & & 7 \\
\hline 19 & THA & & $x$ & & & & & & $x$ & & & & & 2 \\
\hline 20 & UKR & & $x$ & $x$ & & $\mathbf{x}$ & & $x$ & & & $\mathbf{x}$ & & & 5 \\
\hline 21 & USA & & & $\bar{x}$ & $x$ & & $x$ & & $x$ & & & & & 4 \\
\hline 22 & UZB & & & & & & $x$ & & & & $x$ & & $x$ & 3 \\
\hline Insc & samt & & & & & & & & & & & & & 48 \\
\hline
\end{tabular}

\section{Verteilung der Boxer nach Kategorien und Ländern im Finale}

\begin{tabular}{|c|c|c|c|c|c|c|c|c|c|c|c|c|c|c|}
\hline \multicolumn{2}{|c|}{$\begin{array}{l}\text { Gewicht } \\
\text { Land }\end{array}$} & \multirow[t]{2}{*}{$\frac{\vec{D}}{\hat{0}}$} & \multirow[t]{2}{*}{$\stackrel{\overrightarrow{\hat{\theta}}}{\vec{\theta}}$} & \multirow[t]{2}{*}{$\begin{array}{l}\text { Gr } \\
\stackrel{A}{\hat{\theta}}\end{array}$} & \multirow[t]{2}{*}{ ণิ } & \multirow[t]{2}{*}{ 읏 } & \multirow[t]{2}{*}{$\begin{array}{l}\stackrel{ి}{\omega} \\
\text { on } \\
\hat{\hat{a}}\end{array}$} & \multirow[t]{2}{*}{$\frac{\text { ڤ્) }}{\text { ลे }}$} & \multirow[t]{2}{*}{$\stackrel{\overrightarrow{\hat{A}}}{\overrightarrow{\hat{\theta}}}$} & \multirow[t]{2}{*}{ जั } & \multirow{2}{*}{$\begin{array}{l}\stackrel{\infty}{\overrightarrow{\hat{\theta}}} \\
\mathbf{x} \\
\end{array}$} & \multirow[t]{2}{*}{ 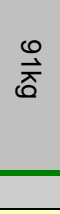 } & \multirow[t]{2}{*}{$\stackrel{\oplus}{\stackrel{+}{\hat{\theta}}}$} & \multirow{2}{*}{ 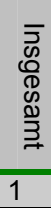 } \\
\hline 1 & CSE & & & & & & & & & & & & & \\
\hline 2 & CUB & $x$ & & $x$ & & $x$ & & & & $\mathbf{x}$ & & $\mathbf{x}$ & & 4 \\
\hline 3 & ESR & $\bar{x}$ & & & & & & & & & & & & 1 \\
\hline 4 & FRA & $\mathbf{x}$ & & & & & & & & & & & & 1 \\
\hline 5 & GBR & & & & & & & & & & & & $x$ & 1 \\
\hline 6 & KAZ & & $\mathbf{x}$ & & $x$ & & & & $x$ & & & & $x$ & 4 \\
\hline 7 & ROM & & & & & & & & $\mathrm{x}$ & & & & & 1 \\
\hline 8 & RUS & & & $x$ & & & & $x$ & & $\mathbf{x}$ & $x$ & $x$ & & 5 \\
\hline 9 & THA & & $x$ & & & & & & & & & & & 1 \\
\hline 10 & UKR & & & & & $x$ & & $x$ & & & & & & 2 \\
\hline 11 & USA & & & & $x$ & & $x$ & & & & & & & 2 \\
\hline 12 & UZB & & & & & & $x$ & & & & & & & 1 \\
\hline Inss & samt & & & & & & & & & & & & & 24 \\
\hline
\end{tabular}




\section{Die Auslosung bei Sydney 2000}

Halbfliegengewicht- bis $48 \mathrm{~kg}$,

\begin{tabular}{|c|c|c|c|c|c|c|}
\hline \multirow{3}{*}{48} & & 117-Sep & $2 \mathrm{Sap}$ & tosep & 28-Sap & $3[\operatorname{Sep}$ \\
\hline & & & & & & \\
\hline & & 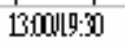 & 13001930 & 13:0019.30 & 1930 & 1300 \\
\hline \multirow{3}{*}{ Damble letto } & & & & & & \\
\hline & & PिEI & \multirow[b]{2}{*}{ EP, $17-15$} & & & \\
\hline & & & & & & \\
\hline \multirow[t]{2}{*}{ Ráád Lzano } & & EMP & & & & \\
\hline & & & & SP, Il-10 & & \\
\hline \multirow[t]{2}{*}{ Mahtide Phuriaile } & & $\mathrm{HSA}$ & & & & \\
\hline & & & KEET, $\mathrm{RSCD}$, & & & \\
\hline \multirow[t]{2}{*}{ Bildi Solmiminain } & & KTW & & & & \\
\hline & & & & & $\mathrm{NP}_{1}, 15 \mathrm{~L}$ & \\
\hline \multirow[t]{2}{*}{ Pal Laktos } & & Holts & & & & \\
\hline & & & PFK, 218 & & & \\
\hline Gim Un Clenl & PHY & PBK, 19-4 & & & & \\
\hline Kelats Seboniso & $\mathrm{LES}$ & & & PHEMIII & & \\
\hline Libonio Romego & $M \mathbb{M E X}$ & $\mathrm{MEX}_{\mathrm{N}}$ L6.15 & & & & \\
\hline Solteri (Webuetek & $\mathrm{kLG}$ & & LTTJ,24]1 & & & \\
\hline Givilo Krtanted & TUGP. & LJU, $9-3$ & & & & \\
\hline Iran Staponic & LTU & & & & & EHA \\
\hline 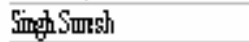 & $\sqrt{4 \mathrm{ND}}$ & KOR 9.5 & & & & \\
\hline EiSook Kim & $\mathrm{KOR}$ & & KCOR \&4 & & & \\
\hline LaP pase Masua & Ints & teth, 10.5 & & & & \\
\hline Iran Calderon & PIR & & & FHS 128 & & \\
\hline 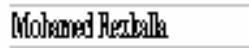 & EST & $F_{R,} 123$ & & & & \\
\hline Brathim Asloum & $F H_{2}$ & & $\mathrm{ER}, 64$ & & & \\
\hline Senguei Kazakor & $\mathrm{B}[\mathrm{SS}$ & USE, \& 6 & & & & \\
\hline Brian טvilonia & TSSA & & & & IRt, [3-12 & \\
\hline Yuldakier Lilitod & TUZ & THस $16-6$ & & & & \\
\hline PmunaStan & THE & & [DRR, , ,WI, I & & & \\
\hline ValenySidorenkn & TRR & UKR, 12-? & & & & \\
\hline Jose Abucuarque & BRs & & & $\mathrm{CIH}, 125$ & & \\
\hline Jose Varela & PQE⿰亻弋口木 & COR, LJ-1 & & & & \\
\hline Wakơs Rameno Esquirol & CIJ & & $\mathrm{COUB}_{1} \mathrm{BSCO}_{4}$ & & & \\
\hline Ranazan Ballinglu & $\mathrm{TCR}$ & $\mathrm{HOM}_{1} \mathrm{x-1}$ & & & & \\
\hline Marian 'velicu & $\mathrm{BOM}$ & & & & & \\
\hline
\end{tabular}

(Deutscher Amateur-Box-Verband in Kassel) 
Fliegengewicht- bis $51 \mathrm{~kg}$,

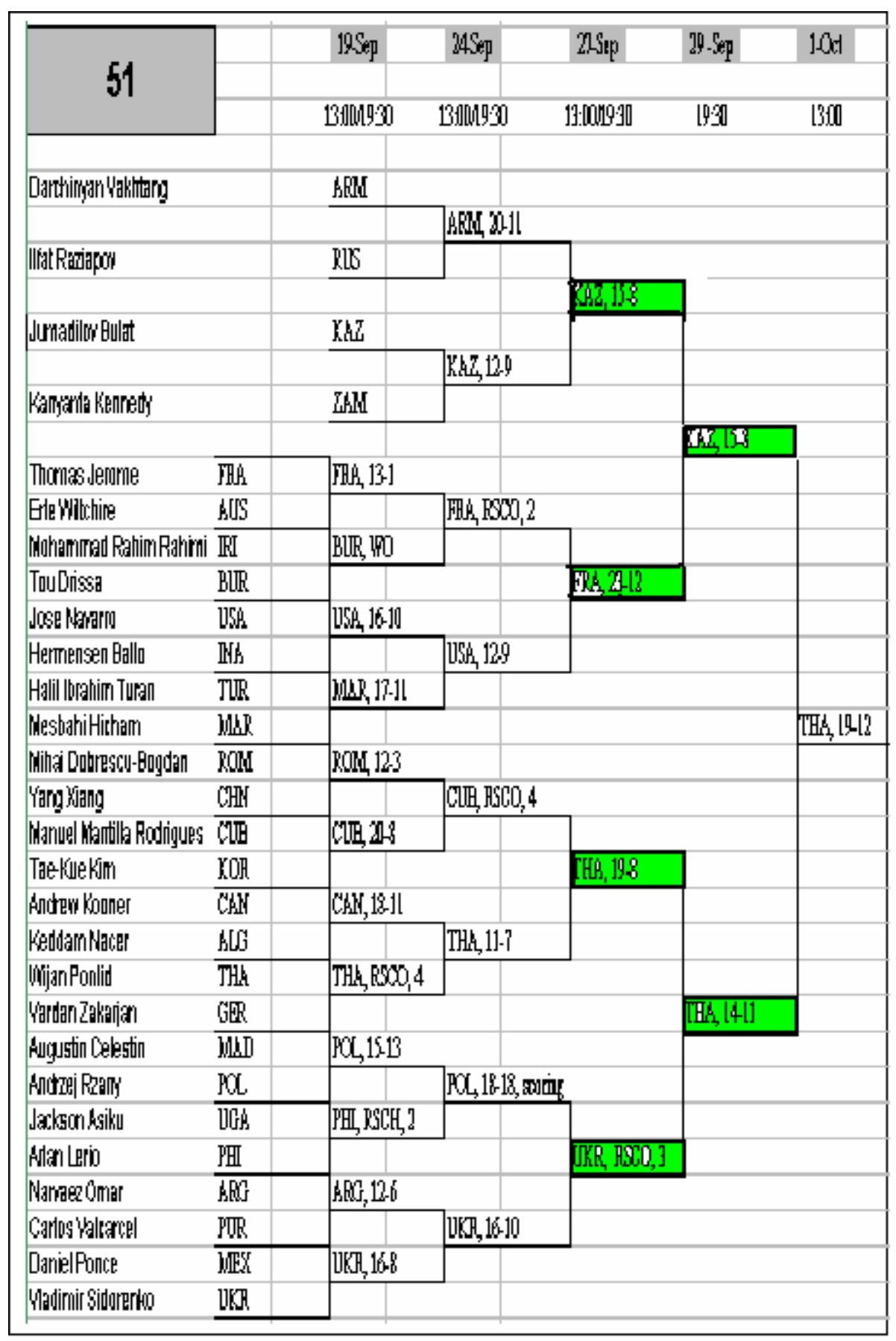

(Deutscher Amateur-Box-Verband in Kassel) 


\section{Bantamgewicht- bis $54 \mathbf{k g}$}

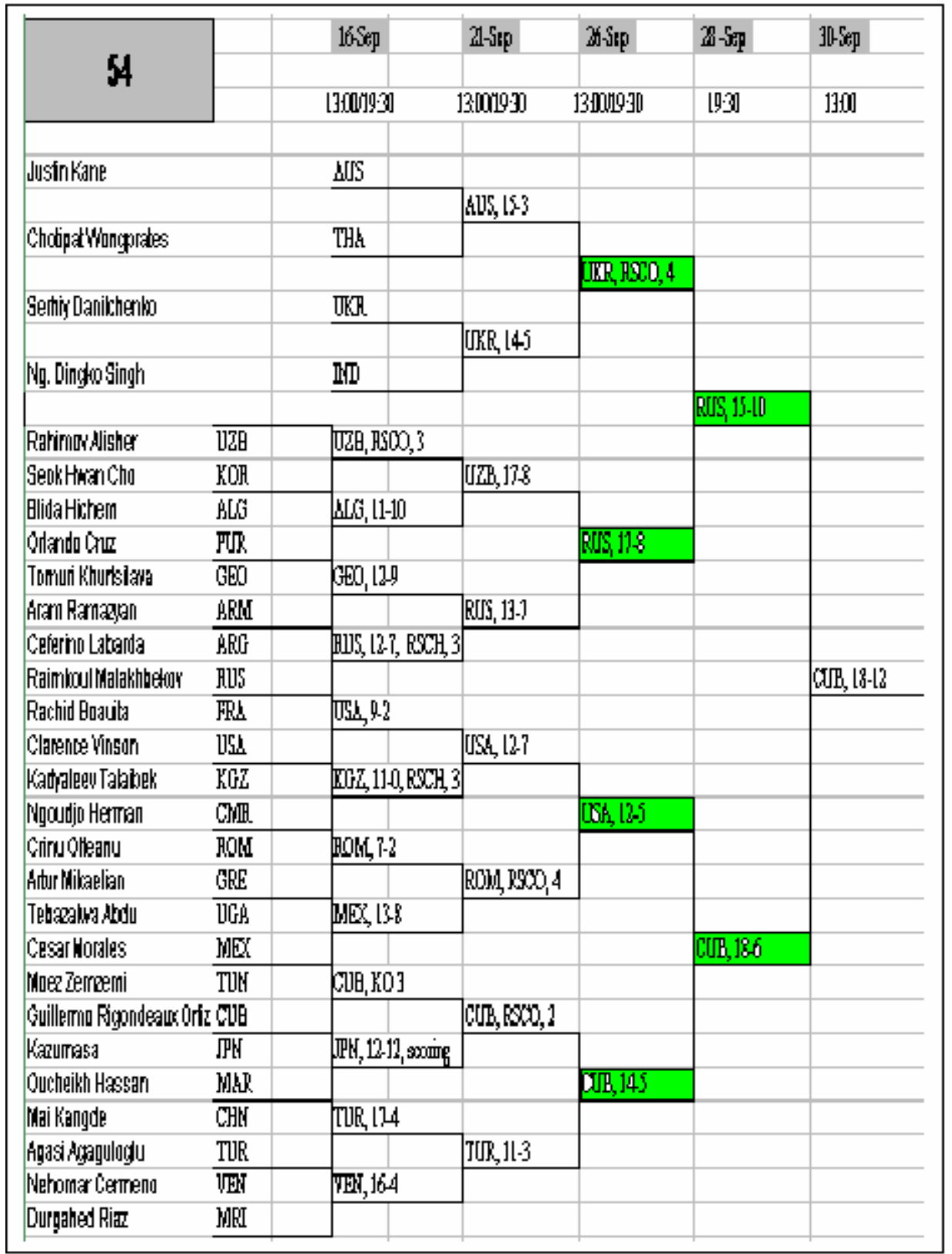

(Deutscher Amateur-Box-Verband in Kassel) 
Federgewicht- bis $57 \mathrm{~kg}$,

\begin{tabular}{|c|c|c|c|c|c|c|c|c|}
\hline & \multirow{3}{*}{57} & & | 10.5epen & BSep & 27.sepp & $2 \operatorname{sep}$ & \multirow{4}{*}{ 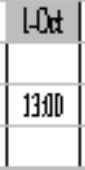 } & \\
\hline & & & & & & \multirow{3}{*}{1930} & & \\
\hline & & & 33019090 & Limang: & $130019: 3]$ & & & \\
\hline & & & & & & & & \\
\hline \multirow[t]{2}{*}{ SWI } & |Wusa Simelane & & $5 \mathrm{KR}$ & & & & & \\
\hline & & & & $\mathrm{ANOS}_{1}, \mathrm{HSOO}_{2}$ & & & & \\
\hline \multirow[t]{2}{*}{$2 F$} & |stael Hectox Perez & & ANAS & & \multirow[b]{2}{*}{$\mathbb{M L R}, 21-18$} & & & \\
\hline & & & & & & & & \\
\hline \multirow[t]{2}{*}{ KAR } & Tamsamani Tahar & & WiR & & & & & \\
\hline & & & & MiLR, 1$] \cdot 14$ & & & & \\
\hline \multirow[t]{2}{*}{$\mathrm{KOR}$} & Pank Heunglihin & & $\mathrm{XOH}$ & & & & & \\
\hline & & & & & & $8 \mathrm{kz}, 2 \mathrm{20}$ & & \\
\hline ROM & Bobinat Misiu Theriu & Fiok & xom 145 & & & & & \\
\hline WW & 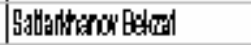 & $\mathrm{N} \mathrm{ML}$ & & XMi lo.5 & & & & \\
\hline $\mathrm{ALG}$ & |Hedthoud Noreddine & HifG & $\mathrm{XSH}, 10.5$ & & & & & \\
\hline B⿵人 & Wathedula bettey & $\mathrm{PSH}$ & & & 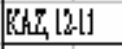 & & & \\
\hline ERA & Dos SantusPereiraYyaldernir & $\mathrm{PRt}$ & 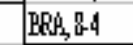 & & & & & \\
\hline $\mathrm{H} M \mathrm{~S}$ & James Swan & $B S$ & & ToR, 1900,3 & & & & \\
\hline$P A K$ & Heidger Ali & PPS & TIR, $5-4$ & & & & & \\
\hline TUR & Rarnazenn Pakani & IITR & & & & & \multicolumn{2}{|c|}{ For 2114} \\
\hline GQR & FakHuste & Gith & GER, L L6 6 & & & & & \\
\hline FN & Joni Tưunent & 跑 & & 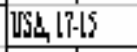 & & & & \\
\hline F & Bijan日atmani & 相] & LEk, $\operatorname{LSC}_{4} 3$ & & & & & \\
\hline WBA & Ricando dae? & Lex & & & {$\left[\mathrm{CS}_{1}, \mathrm{BSCO}, 4\right.$} & & & \\
\hline 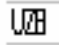 & |Tugunnor Tukunbrap & $\mathrm{UTH}$ & uX? 12$]$ & & & & & \\
\hline UGA & Wapa Atamkassim & bas & & T愳, ?-2 & & & & \\
\hline THA & |Kansing Sombsk & $\mathbb{I T H}$ & $\mathrm{THE}, 1,2$ & & & & & \\
\hline$\omega \mathrm{LL}$ & Andres Abe ino Lederama & bol & & & & $\pi S_{1}, 2-12$ & & \\
\hline DWR & Senin Bdeg,manor & UWRE. & B⿺辶斤 74 & \multirow[b]{2}{*}{ Cण } & & & & \\
\hline BUL & Jui hlladenory & $\mathbb{P}[\mathrm{L}$ & & & & & & \\
\hline JPN & Hideriko Tskamoti & $\mathrm{PPN}$ & ats, & & & & & \\
\hline Cu日 & Yoskay/Aguilen Zamorf & बत् & & & $2[5,12,1]$ & & & \\
\hline RUSS & |Karril Djamabujinor & $\mathrm{ATSS}$ & $\mathrm{N}[\mathrm{J}, 0.5$ & & & & & \\
\hline $\mathrm{LTN}$ & |Gicjulatas Vitas & LIJ & & $\mathrm{ROS}, 15-12$ & & & & \\
\hline ETH & Shefreraw Yohannes & Bill & $\mathrm{WEX}, 8 \mathrm{BC}, 4$ & & & & & \\
\hline $\mathbb{M E x}$ & Franciso Bojab Aados & $\sqrt{W}$ & & & & & & \\
\hline
\end{tabular}

(Deutscher Amateur-Box-Verband in Kassel) 
Leichtgewicht- bis $60 \mathrm{~kg}$,

\begin{tabular}{|c|c|c|c|c|c|c|}
\hline & & 17Sep & 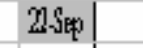 & 26. & IRSp & $x$ Sep \\
\hline & & 13001 & 130m4-10 & 170090 & $10 \%$ & 1300 \\
\hline & & & & & $0-\infty$ & \\
\hline \multirow{3}{*}{ Bed Asgar Alighah } & & & & & & \\
\hline & & PHK & & & & \\
\hline & & & GHE, L'LL'5 & & & \\
\hline \multirow[t]{2}{*}{ Tigran 0ுdian } & & GRE & & & & \\
\hline & & & & $\mathrm{CDQ}, \mathrm{BSOO}, 4$ & & \\
\hline \multirow[t]{2}{*}{ Hanio Kjndelan Hesa } & & Cug & & & & \\
\hline & & & CСТR, 148 & & & \\
\hline \multirow[t]{2}{*}{ Pongsillyiargrisas } & & THAk & & & & \\
\hline & & & & & , & \\
\hline Nonmian Schuser & P政. & VWW, 24]10 & & & & \\
\hline PatickLoper & FEY & & $\mathrm{B}[\mathrm{S}, \mathrm{BO}, 3$ & & & \\
\hline AJexandre Haein & ETSS & Fus, 145 & & & & \\
\hline Holthach Nuridinor & tw不 & & & $\mathrm{HOJ}_{3}, \mathrm{BSCO}, 4$ & & \\
\hline Naoufeal Nuniddring & [T]] & 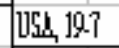 & & & & \\
\hline Daid Jackson & ISSA & & TCT, whous & & & \\
\hline SelimPaliani & TTR & TUR, L4j & & & & \\
\hline Âtodel Jebati & ERA & & & & & Cot, 144 \\
\hline Hichæalkadsidis & kllJS & $\mathrm{B}_{1}\left[\coprod_{1}, 156\right.$ & & & & \\
\hline Aggnaldo Wagalhaes & $\mathbb{E R}$ & & $\mathrm{Kh} 2,9-1$ & & & \\
\hline Âtur Qenolijan & kHWd & Keद $16-6$ & & & & \\
\hline 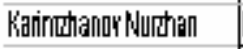 & $\mathrm{KAZ}$ & & & DIRH, 1500,3 & & \\
\hline Laty Semillano & FHI & DUKR, 16] & & & & \\
\hline Andres Kotehik & [TRR & & {$[\mathrm{ER}, 1]-[1$} & & & \\
\hline VícorRamos & {$[0 \mathrm{k}$} & $\operatorname{set}_{3}, 150$ & & & & \\
\hline Nath Ragminand & PHg & & & & [NR $2-14$ & \\
\hline Geome Lungu & (BD)M & R0L, 153 & & & & \\
\hline 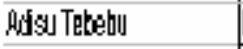 & ETH & & (4W & & & \\
\hline Cristian Bejarano Benilez & MEX & $\mathrm{MEX}, \mathrm{L}-5$ & & & & \\
\hline Khurrikane Gillbet & BOT & & & $M E X_{1} 1412$ & & \\
\hline 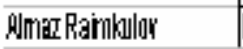 & KGG & $\mathrm{Y}(\mathrm{CZ}, 154$ & & & & \\
\hline Iunentistsegs ldiunten & MOLL & & {$[G 2,112$} & & & \\
\hline Jose Lenando Cno Lam & $100 \mathrm{~L}$ & WLL, 10.9 & & & & \\
\hline Frontin Govanri Kíchael & $\mathbb{W R}$ & & & & & \\
\hline
\end{tabular}

(Deutscher Amateur-Box-Verband in Kassel) 
Halbweltergewicht- bis $63,5 \mathrm{~kg}$,

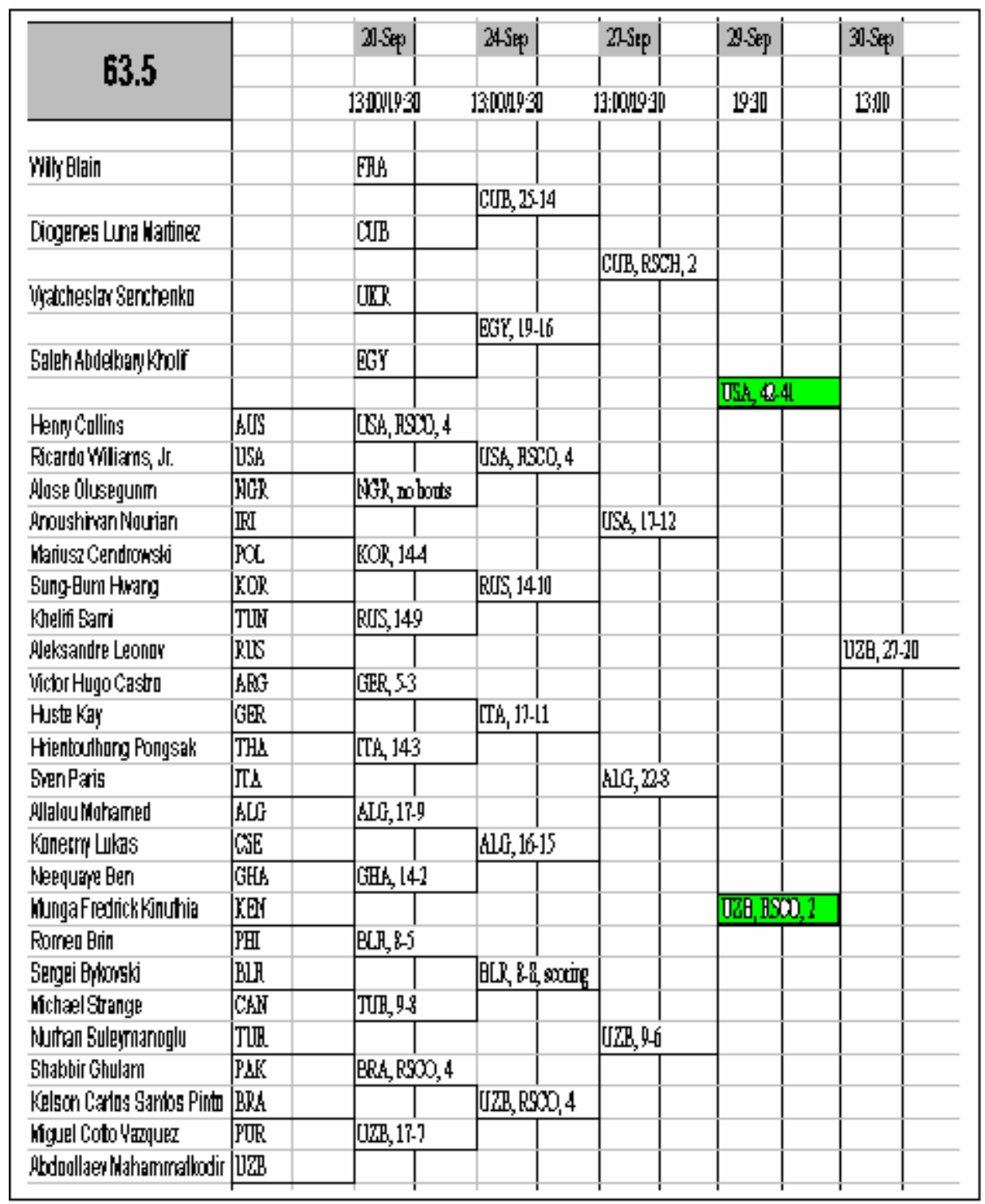

(Deutscher Amateur-Box-Verband in Kassel) 


\section{Weltergewicht- bis $67 \mathrm{~kg}$,}

\begin{tabular}{|c|c|c|c|c|c|c|}
\hline & & 16-Sep & 2lsep & 35.epp & 10.Sep & nosep \\
\hline & & $1300 \mathrm{n} 0.21$ & 1300110.8 & 13009010 & 10.40 & 130 \\
\hline & & & & & & \\
\hline \multirow[t]{2}{*}{ KamelChaler } & & TOS & & & & \\
\hline & & & 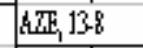 & & & \\
\hline \multirow[t]{2}{*}{ Ruslan Khairơ } & & hIE & & & & \\
\hline & & & & Ho, 10,10 & & \\
\hline \multirow[t]{2}{*}{ Okg gatory } & & BDS & & & & \\
\hline & & & EJS, LS-l & & & \\
\hline \multirow[t]{2}{*}{ Frantiswo Calderon } & & $\infty \mathrm{L}$ & & & & \\
\hline & & & & & D]J, $19-11$ & \\
\hline Chibuse Ellis & $\mathrm{ZB} B \mathrm{MK}$ & $\operatorname{Con}, 1 \mathrm{R}^{-4}$ & & & & \\
\hline Robeto Guenra Rirerto & CuB & & BOM, 11-7 & & & \\
\hline Ruben Fuchu & गॉ & HOM, BSC3 & & & & \\
\hline Simion Dorel & ROM & & & Holu, $26 \cdot 14$ & & \\
\hline Tsie hosolesa & $\mathrm{IF}$ & GER ASC1 & & & & \\
\hline Steven Kuschler & GEH & & GER, BOO, 3 & & & \\
\hline Lorenzo Ýxanrif & $\overline{\mathrm{DOM}}$ & Dom, 54 & & & & \\
\hline Usman U\|lah & PkL & & & & & $\mathrm{HLS}_{3}, 2416$ \\
\hline Geard Higlaic & IJIS & THE, $9-9$, , scond & & & & \\
\hline Jargphorak Partquoun & THA & & [ [ & & & \\
\hline Guillermo Saputm & BRRS & $\mathrm{DKR}, 12 ?$ & & & & \\
\hline Sergep Dodseriko & UKCH. & & & $\mathrm{UETH}, 8 ?$ & & \\
\hline DanielGeale & $\overline{\mathrm{A}}[\mathrm{SS}$ & ITS 42 & & & & \\
\hline Learanda Bundi & ] Tlk & & $\log , 134$ & & & \\
\hline Numumbermbe Paudus & HitM & IKAZ, 142 & & & & \\
\hline Munailbascr Danijar & KLW & & & & $\mathbb{K M}, 128$ & \\
\hline Bulent Uusoi & TUR & TणU, $8-6$ & & & & \\
\hline Bae Jin Seak & $\mathrm{KOH}$ & & T[CH, 94 & & & \\
\hline ShountanFadel & $\mathrm{BgY}$ & DSt, $\mathrm{BSO}_{1} 4$ & & & & \\
\hline Donte Craig & USk & & & $\mathrm{MDC}, 10-1$ & & \\
\hline Mogimi Babak & $\overline{\mathrm{JR}}$ & $\mathrm{DZR}, 1<\mathrm{j}$ & & & & \\
\hline Sherood Husanor & UZB & & twas, 13-7 & & & \\
\hline Tsegasellase Aregainj & ETH & $M D k, 10$ bot & & & & \\
\hline Vítalie Grusac: & $\mathrm{MDA}$ & & & & & \\
\hline
\end{tabular}

(Deutscher Amateur-Box-Verband in Kassel) 
Halbmittelgewicht- bis $71 \mathrm{~kg}$,

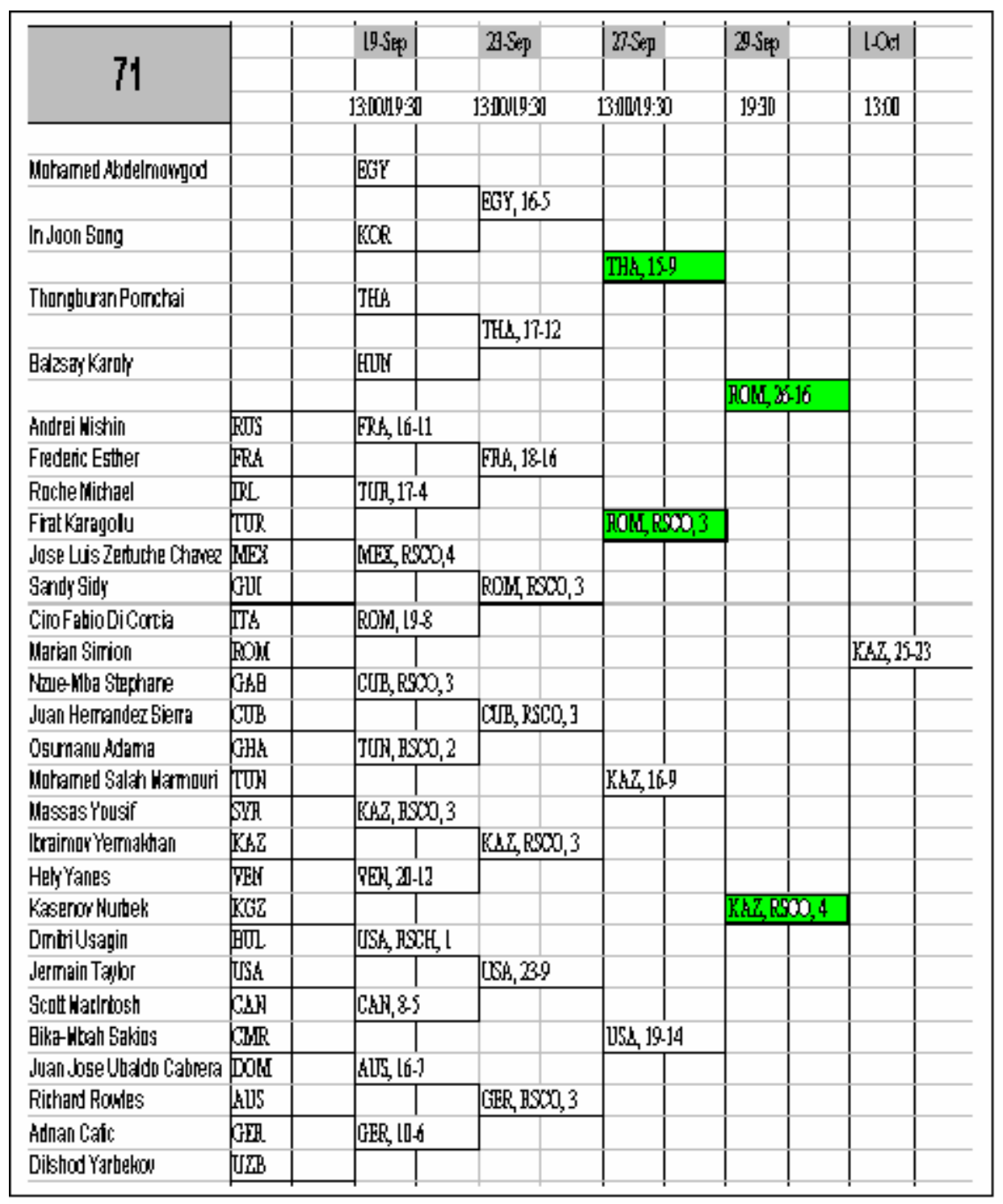

(Deutscher Amateur-Box-Verband in Kassel) 
Mittelgewicht- bis $75 \mathrm{~kg}$,

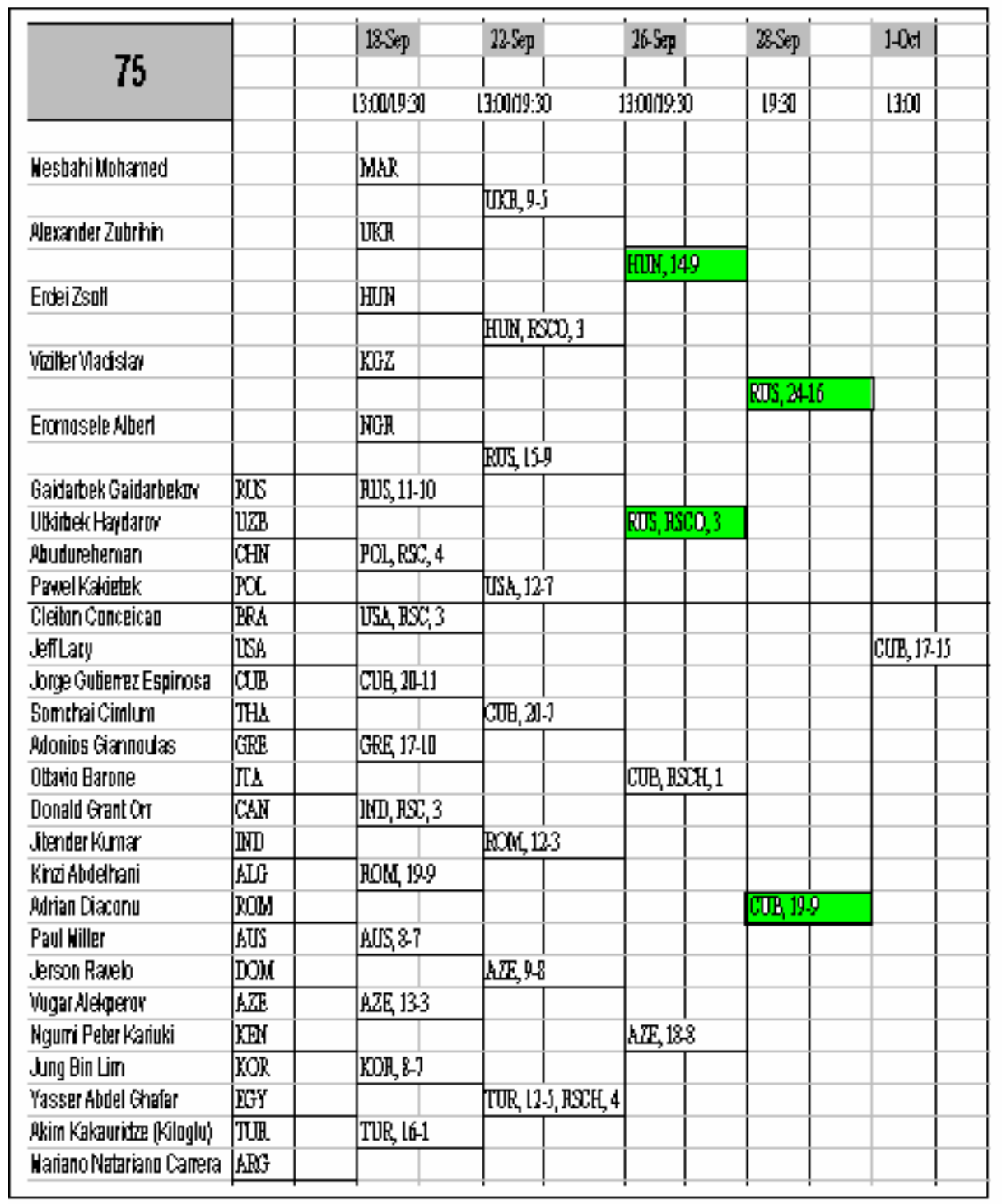

(Deutscher Amateur-Box-Verband in Kassel) 
Halbschwergewicht- bis $81 \mathrm{~kg}$,

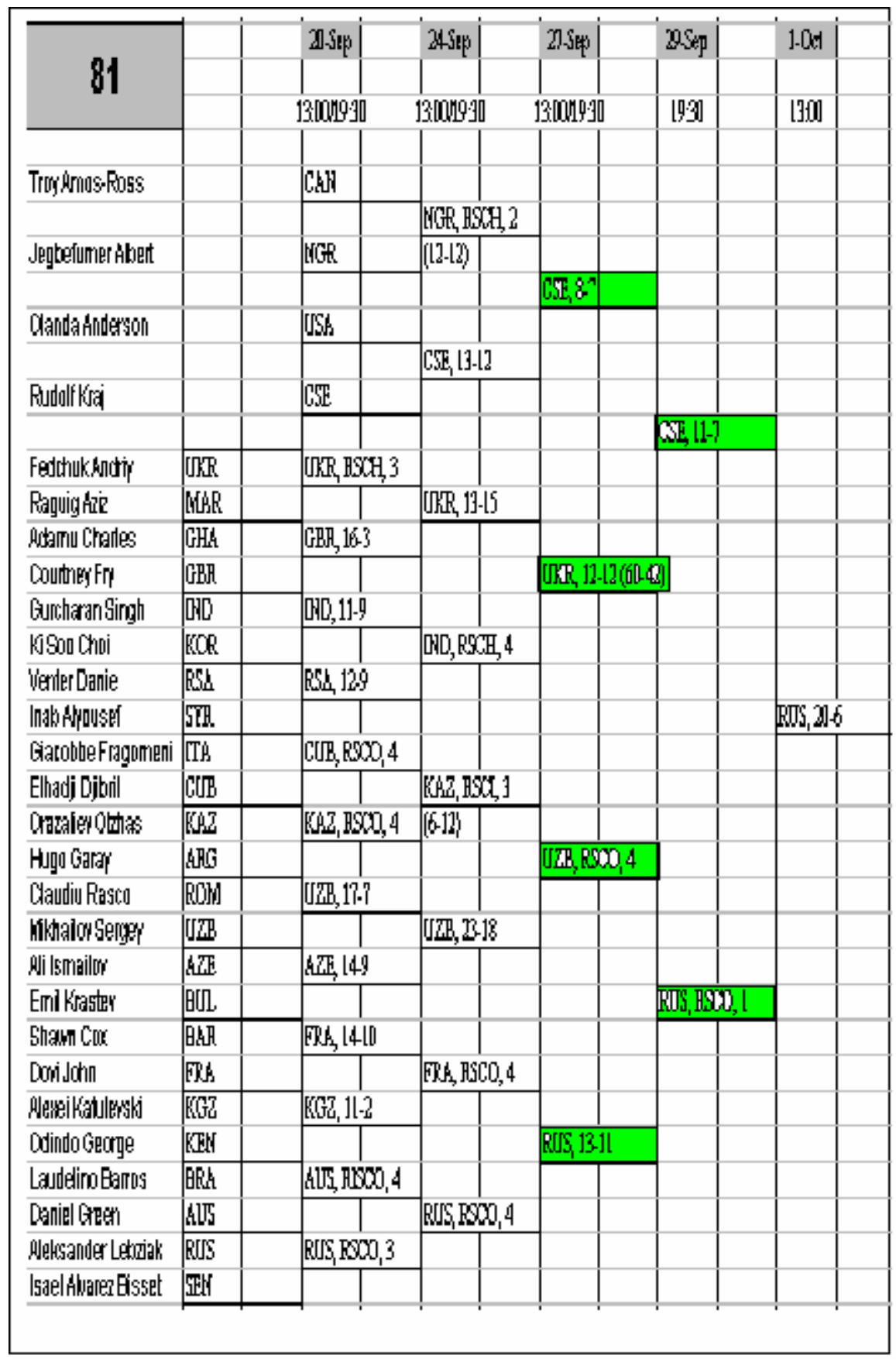

(Deutscher Amateur-Box-Verband in Kassel) 
Schwergewicht- bis $91 \mathrm{~kg}$,

\begin{tabular}{|c|c|c|c|c|c|}
\hline \multirow{3}{*}{91} & & 21.Sep & 26 Sep & IEsep & 3lsep \\
\hline & & & & & \\
\hline & & 13:0019:30 & 130019:90 & $19: 30$ & 1300 \\
\hline & & & & & \\
\hline \multirow[t]{2}{*}{ Wark Simmons } & CBN & & & & \\
\hline & & $\mathrm{Car}_{1} \mathrm{~L}-6$ & & & \\
\hline \multirow[t]{2}{*}{ Hoseini Rohoullah } & EII & & & & \\
\hline & & & GER RSCO 3 & & \\
\hline \multirow[t]{2}{*}{ Nagamed Aripgadjiver } & $A Z E$ & & & & \\
\hline & & GER 9.4 & & & \\
\hline \multirow[t]{2}{*}{ Sebastian Koeber } & GER & & & & \\
\hline & & & & CJU, 108 & \\
\hline \multirow{2}{*}{ Ojemaye Rasmus } & WOOR & & & & \\
\hline & & COH, RSO & & & \\
\hline \multirow[t]{2}{*}{ Félin Stroun Fabre } & CTOB & & & & \\
\hline & & & $\mathrm{COB}, \mathrm{RSCO}$ & & \\
\hline \multirow{2}{*}{ Wiojojiech Barthik } & $\mathrm{POL}$ & & & & \\
\hline & & ISS , 11.2 & & & \\
\hline \multirow[t]{2}{*}{ Nichael Bennett } & [OSB & & & & \\
\hline & & & & & $\mathrm{CUB}, 21-1$ \\
\hline \multirow[t]{2}{*}{ Yadimir Tschantouria } & GEO & & & & \\
\hline & & GEO,RSO & & & \\
\hline \multirow[t]{2}{*}{ Anno Mustafa Mahmoud } & EGY & & & & \\
\hline & & & $380,[8-1]$ & & \\
\hline \multirow[t]{2}{*}{ Chagaeviruslan } & UTZB & & & & \\
\hline & & UUZW, 15.2 & & & \\
\hline \multirow[t]{2}{*}{ Aestandr Yalsenko } & LOKR & & & & \\
\hline & & & & RIS, 194 & \\
\hline \multirow[t]{2}{*}{ Azaoui Mohanted } & ths & & & & \\
\hline & & FRA RSC & & & \\
\hline \multirow[t]{2}{*}{ Jackson Chanet } & FRs & & & & \\
\hline & & & $\operatorname{ADS}, 1 \mathrm{~L}-13$ & & \\
\hline \multirow[t]{2}{*}{ Pauga Lalau } & SAM & & & & \\
\hline & & RIS, BSC & & & \\
\hline Soultanakhimed lbraguimor & RUIS & & & & \\
\hline
\end{tabular}

(Deutscher Amateur-Box-Verband in Kassel) 
Superschwergewicht- über $91 \mathrm{~kg}$,

\begin{tabular}{|c|c|c|c|c|c|}
\hline \multirow{3}{*}{$91+$} & & RSep & 27.Sep & 29.Sep & $1.0 \mathrm{ct}$ \\
\hline & & \multirow[b]{2}{*}{ 13:0019:30 } & & & \\
\hline & & & 1301990 & $19: 30$ & $13: 00$ \\
\hline \multirow{2}{*}{ Ahrned lurahim } & Fir & & & & \\
\hline & & UZH, 21-8 & & & \\
\hline \multirow[t]{2}{*}{ Saidu Rustarn } & UZH & & & & \\
\hline & & & $\mathrm{UZZ}, \mathrm{BSOO}$, & & \\
\hline \multirow[t]{2}{*}{ Arthur Binhouski } & Cêt & & & & \\
\hline & & CANH, 21-14 & & & \\
\hline \multirow[t]{2}{*}{ Nacaque Nichael } & MRI & & & & \\
\hline & & & & FAZ,20-21 & \\
\hline \multirow[t]{2}{*}{ Wukhtarthan Dildabehou } & $\mathrm{KaZ}$ & & & & \\
\hline & & X\&Z, 16.5 & & & \\
\hline \multirow[t]{2}{*}{ Kielsa Gregor } & POL & & & & \\
\hline & & & Ka, 25-12 & & \\
\hline \multirow[t]{2}{*}{ Alexs Rubalcaba Polledo } & $\mathrm{CJH}$ & & & & \\
\hline & & $\mathrm{ClUH}, \mathrm{HSCH}, 1$ & & & \\
\hline \multirow[t]{2}{*}{ Cengi $\mathrm{K}_{00}$} & GER & & & & \\
\hline & & & & & OBR, 30-L6 \\
\hline \multirow[t]{2}{*}{ Constantin Onofrei } & $\mathrm{ROMK}$ & & & & \\
\hline & & WGß, 17.14 & & & \\
\hline \multirow[t]{2}{*}{ PeterSamuel } & IIGR & & & & \\
\hline & & & TTh, 143 & & \\
\hline \multirow[t]{2}{*}{ Panlo víddar } & ITs & & & & \\
\hline & & $\mathrm{ITA}, \mathrm{RSOO}, 4$ & & & \\
\hline \multirow[t]{2}{*}{ Caluin Bnock } & USA & & & & \\
\hline & & & & GBR, 3216 & \\
\hline \multirow[t]{2}{*}{ Harrison Hudley } & GBR & & & & \\
\hline & & GBR, RSCH, 4 & & & \\
\hline \multirow[t]{2}{*}{ Alexei Lezin } & $\mathrm{R} J \mathrm{SS}$ & & & & \\
\hline & & & G8R, 19.9 & & \\
\hline \multirow{2}{*}{ Angus Whare Shelford } & $\mathrm{HZZ}$ & & & & \\
\hline & & ЈКЖ, 10.5 & & & \\
\hline Oleksii Hajkin & TKH. & & & & \\
\hline
\end{tabular}

(Deutscher Amateur-Box-Verband in Kassel) 


\section{Formular für die Analysesysteme für jeden Kampf}

Die Wettkämpfe: (Halbfinale oder Finale) Gewichtsklasse ( ） Kampfnummer: ( )

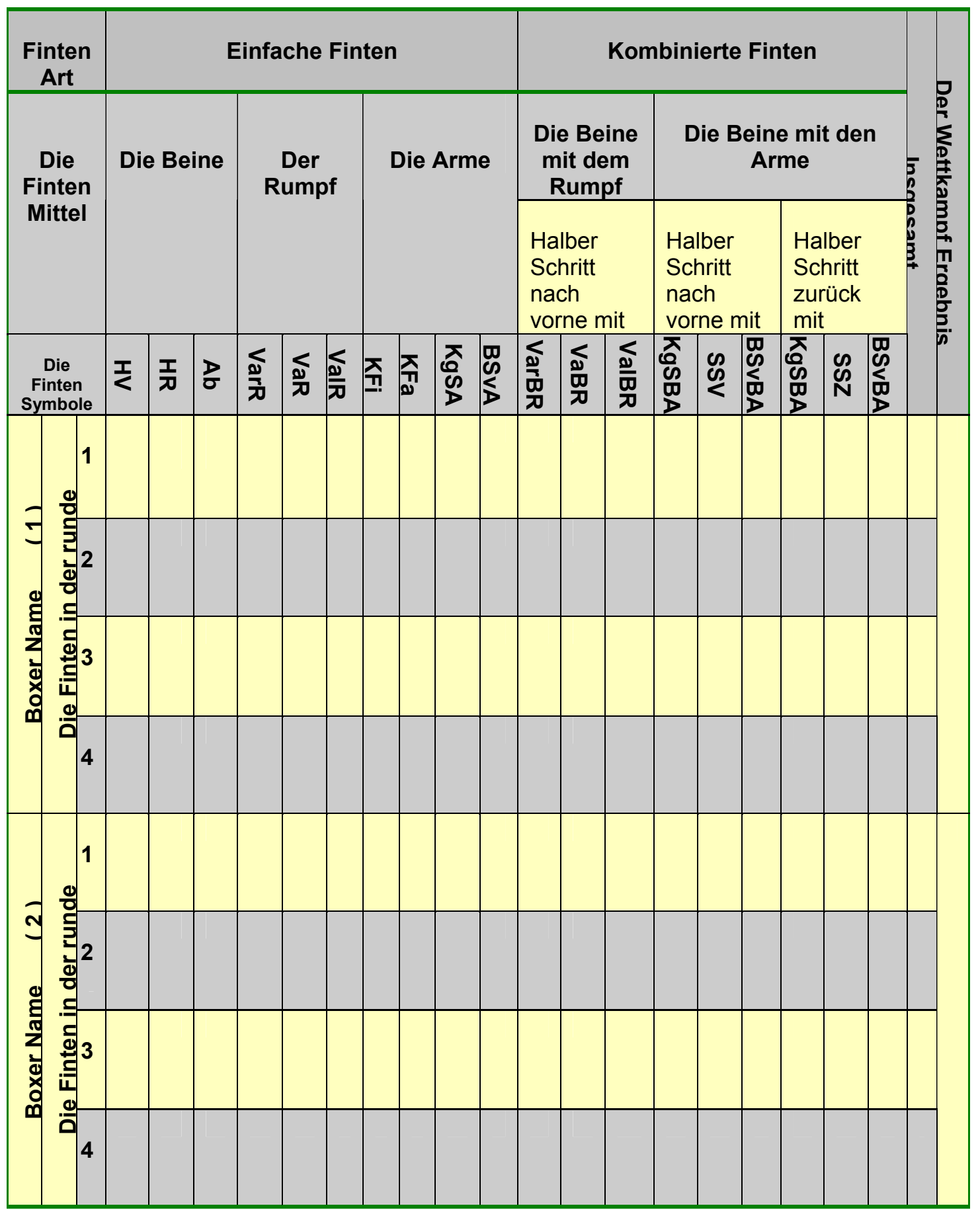




\section{Medaillenspiegel für der Sieger}

Boxen - 48kg - Finale

\begin{tabular}{|l|l|l|}
\hline Rang & Name & Land \\
\hline Gold & Brahim Asloum & Frankreich \\
\hline Silber & Rafael Lozano & Spanien \\
\hline Bronze & Maikro Romero & Kuba \\
\hline Bronze & Un Chol Kim & Nord Korea \\
\hline
\end{tabular}

Boxen - 51kg - Finale

\begin{tabular}{|l|l|l|}
\hline Rang & Name & Land \\
\hline Gold & Wijan Ponlid & Thailand \\
\hline Silber & Bulat Jumadilov & Kasachstan \\
\hline Bronze & Jerome Thomas & Frankreich \\
\hline Bronze & Volodymyr Sydorenko & Ukraine \\
\hline
\end{tabular}

Boxen - 54kg - Finale

\begin{tabular}{|l|l|l|}
\hline Rang & Name & Land \\
\hline Gold & Guillermo Rigondeaux & Kuba \\
\hline Silber & Raimkoul Malakhbekov & Rußland \\
\hline Bronze & Sergiy Danylchenko & Ukraine \\
\hline Bronze & Clarence Vinson & USA \\
\hline
\end{tabular}

Boxen - 57kg - Finale

\begin{tabular}{|l|l|l|}
\hline Rang & Name & Land \\
\hline Gold & Bekzat Sattarkhanov & Kasachstan \\
\hline Silber & Ricardo Juarez & USA \\
\hline Bronze & Tahar Tamsamani & Marokko \\
\hline Bronze & Kamil Djamaloudinov & Rußland \\
\hline
\end{tabular}


Boxen - 60kg - Finale

\begin{tabular}{|l|l|l|}
\hline Rang & Name & Land \\
\hline Gold & Mario Kindelan & Kuba \\
\hline Silber & Andriy Kotelnyk & Ukraine \\
\hline Bronze & Cristian Bejarano & Mexiko \\
\hline Bronze & Alexandre Maletine & Rußland \\
\hline
\end{tabular}

Boxen - 63,5kg - Finale

\begin{tabular}{|l|l|l|}
\hline Rang & Name & Land \\
\hline Gold & Mahammatkodir Abdoollayev & Uzbekistan \\
\hline Silber & Ricardo Williams Jr & USA \\
\hline Bronze & Mohamed Allalou & Algerien \\
\hline Bronze & Diogenes Luna & Kuba \\
\hline
\end{tabular}

Boxen - 67kg - Finale

\begin{tabular}{|l|l|l|}
\hline Rang & Name & Land \\
\hline Gold & Oleg Saitov & Rußland \\
\hline Silber & Sergiy Dotsenko & Ukraine \\
\cline { 2 - 3 } Bronze & Vitalie Grusac & Moldawien \\
\hline Bronze & Dorel Simion & Rumänien \\
\hline
\end{tabular}

Boxen - 71kg - Finale

\begin{tabular}{|l|l|l|}
\hline Rang & Name & Land \\
\hline Gold & Yermakhan Ibraimov & Kasachstan \\
\hline Silber & Marian Simion & Rumänien \\
\hline Bronze & Pornchai Thongburan & Thailand \\
\hline Bronze & Jermain Taylor & USA \\
\hline
\end{tabular}


Boxen - 75kg - Finale

\begin{tabular}{|l|l|l|}
\hline Rang & Name & Land \\
\hline Gold & Jorge Gutierrez & Kuba \\
\hline Silber & Gaidarbek Gaidarbekov & Rußland \\
\cline { 2 - 3 } Bronze & Vugar Alakparov & Aserbaidschan \\
\hline Bronze & Zsolt Erdei & Ungarn \\
\hline
\end{tabular}

Boxen - 81kg - Finale

\begin{tabular}{|l|l|l|}
\hline Rang & Name & Land \\
\hline Gold & Alexandre Lebziak & Rußland \\
\hline Silber & Rudolf Kraj & $\begin{array}{l}\text { Tschechische Re- } \\
\text { publik }\end{array}$ \\
\hline Bronze & Andriy Fedchuk & Ukraine \\
\hline Bronze & Sergey Mihaylov & Uzbekistan \\
\hline
\end{tabular}

Boxen - 91 kg - Finale

\begin{tabular}{|l|l|l|}
\hline Rang & Name & Land \\
\hline Gold & Felix Savon & Kuba \\
\hline Silber & Soultanakhmed Ibraguimov & Rußland \\
\hline Bronze & Vladimer Tchanturia & Georgien \\
\hline Bronze & Sebastian Koeber & Deutschland \\
\hline
\end{tabular}

Boxen - Über 91kg - Finale

\begin{tabular}{|l|l|l|}
\hline Rang & Name & Land \\
\hline Gold & Audley Harrison & Großbritannien \\
\hline Silber & Mukhtarkhan Dildabekov & Kasachstan \\
\hline Bronze & Paolo Vidoz & Italien \\
\hline Bronze & Rustam Saidov & Uzbekistan \\
\hline
\end{tabular}


9. Medaillenspiegel für die Länder

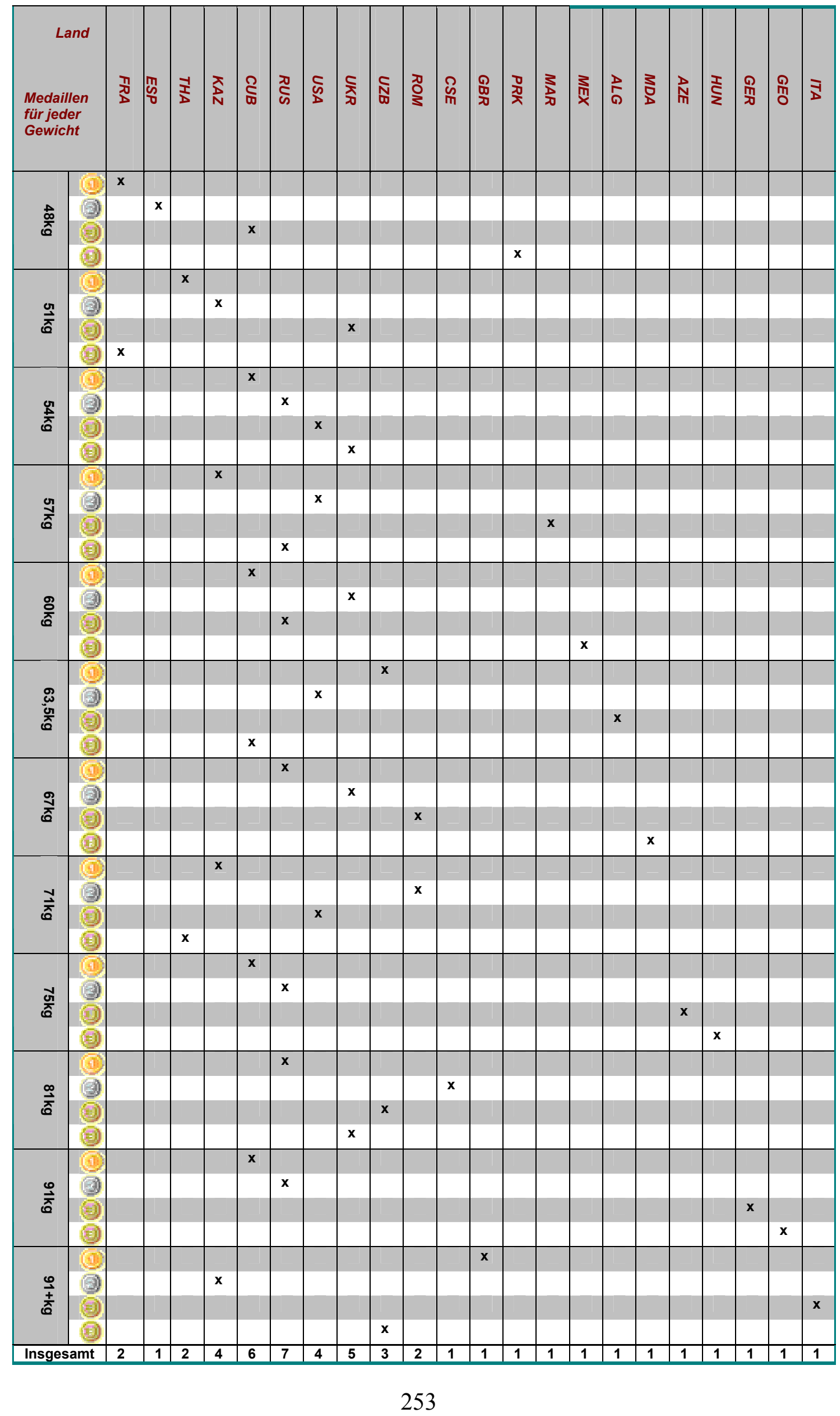




\section{Gewichts- und Alterklassen Amateure (männlich)}

\begin{tabular}{|c|c|c|c|c|c|}
\hline $\begin{array}{l}\text { Gewichtsklas- } \\
\text { senbezeichnung }\end{array}$ & Schüler & Jugend & Kadetten & Junioren & Männer \\
\hline Altersklasse & AK $10-12$ & AK $13-14$ & AK $15-16$ & AK $17-18$ & $\begin{array}{l}\text { AK } 19-30 \\
(37)\end{array}$ \\
\hline Kämpfe pro Jahr & 20 & 20 & 25 & 35 & $\begin{array}{l}\text { ohne Be- } \\
\text { schränkung }\end{array}$ \\
\hline Wettkampfzeit & $3 \times 1 \mathrm{~min}$ & $3 \times 2 \min$ & $3 \times 2 \mathrm{~min}$ & $4 \times 2 \mathrm{~min}$ & $4 \times 2 \min$ \\
\hline $\begin{array}{l}\text { Pause zwischen } \\
\text { den Kämpfen }\end{array}$ & 5 Tage & 5 Tage & 5 Tage & 4 Tage & 4 Tage \\
\hline Papiergewicht & $30 \mathrm{~kg}$ & $30 \mathrm{~kg}$ & & & \\
\hline Papiergewicht & $32 \mathrm{~kg}$ & $32 \mathrm{~kg}$ & & & \\
\hline Papiergewicht & $34 \mathrm{~kg}$ & $34 \mathrm{~kg}$ & & & \\
\hline Papiergewicht & $36 \mathrm{~kg}$ & $36 \mathrm{~kg}$ & & & \\
\hline Papiergewicht & $38 \mathrm{~kg}$ & $38 \mathrm{~kg}$ & & & \\
\hline Papiergewicht & $40 \mathrm{~kg}$ & $40 \mathrm{~kg}$ & $42 \mathrm{~kg}$ & & \\
\hline Papiergewicht & $42 \mathrm{~kg}$ & $42 \mathrm{~kg}$ & $44 \mathrm{~kg}$ & & \\
\hline Papiergewicht & $45 \mathrm{~kg}$ & $45 \mathrm{~kg}$ & $46 \mathrm{~kg}$ & & \\
\hline $\begin{array}{l}\text { Halbfliegenge- } \\
\text { wicht }\end{array}$ & $48 \mathrm{~kg}$ & $48 \mathrm{~kg}$ & $48 \mathrm{~kg}$ & $48 \mathrm{~kg}$ & $48 \mathrm{~kg}$ \\
\hline Fliegengewicht & $51 \mathrm{~kg}$ & $51 \mathrm{~kg}$ & $50 \mathrm{~kg}$ & $51 \mathrm{~kg}$ & $51 \mathrm{~kg}$ \\
\hline Bantamgewicht & $54 \mathrm{~kg}$ & $54 \mathrm{~kg}$ & $52 \mathrm{~kg}$ & $54 \mathrm{~kg}$ & $54 \mathrm{~kg}$ \\
\hline Federgewicht & $57 \mathrm{~kg}$ & $57 \mathrm{~kg}$ & $54 \mathrm{~kg}$ & $57 \mathrm{~kg}$ & $57 \mathrm{~kg}$ \\
\hline Leichtgewicht & $60 \mathrm{~kg}$ & $60 \mathrm{~kg}$ & $57 \mathrm{~kg}$ & $60 \mathrm{~kg}$ & $60 \mathrm{~kg}$ \\
\hline $\begin{array}{l}\text { Halbwelterge- } \\
\text { wicht }\end{array}$ & $63 \mathrm{~kg}$ & $63 \mathrm{~kg}$ & $60 \mathrm{~kg}$ & $64 \mathrm{~kg}$ & $64 \mathrm{~kg}$ \\
\hline Weltergwicht & $66 \mathrm{~kg}$ & $66 \mathrm{~kg}$ & $63 \mathrm{~kg}$ & $69 \mathrm{~kg}$ & $69 \mathrm{~kg}$ \\
\hline $\begin{array}{l}\text { Halbmittelge- } \\
\text { wicht }\end{array}$ & $69 \mathrm{~kg}$ & $69 \mathrm{~kg}$ & $66 \mathrm{~kg}$ & entfällt & entfälltt \\
\hline Mittelgewicht & $72 \mathrm{~kg}$ & $72 \mathrm{~kg}$ & $70 \mathrm{~kg}$ & $75 \mathrm{~kg}$ & $75 \mathrm{~kg}$ \\
\hline $\begin{array}{l}\text { Halbschwerge- } \\
\text { wicht }\end{array}$ & $75 \mathrm{~kg}$ & $75 \mathrm{~kg}$ & $75 \mathrm{~kg}$ & $81 \mathrm{~kg}$ & $81 \mathrm{~kg}$ \\
\hline Schwergewicht & $81 \mathrm{~kg}$ & $81 \mathrm{~kg}$ & $80 \mathrm{~kg}$ & $91 \mathrm{~kg}$ & $91 \mathrm{~kg}$ \\
\hline $\begin{array}{l}\text { Superschwer- } \\
\text { gewicht }\end{array}$ & über. $81 \mathrm{~kg}$ & über. $81 \mathrm{~kg}$ & $86 \mathrm{~kg}$ & über. $91 \mathrm{~kg}$ & über. $91 \mathrm{~kg}$ \\
\hline $\begin{array}{l}\text { Superschwer- } \\
\text { plusgewicht }\end{array}$ & & & über. $86 \mathrm{~kg}$ & & \\
\hline
\end{tabular}

http://www.kontaktrunde.de/boxen/gewichtsklassen.html10.10. 2003, 16:09 Uhr. 


\section{Gewichts- und Alterklassen Amateure (weiblich)}

\begin{tabular}{|l|l|l|l|l|l|}
\hline $\begin{array}{l}\text { Gewichtsklas- } \\
\text { senbezeichnung }\end{array}$ & Jugend C & Jugend B & Jugend A & $\begin{array}{l}\text { Juniorin- } \\
\text { nen }\end{array}$ & Frauen \\
\hline Altersklasse & AK 10 - 12 & AK 13 - 14 & AK 15 - 16 & AK 17 - 18 & AK 19 \\
\hline $\begin{array}{l}\text { Kämpfe pro } \\
\text { Jahr }\end{array}$ & 20 & 20 & 25 & 35 & $\begin{array}{l}\text { ohne Be- } \\
\text { schrän- } \\
\text { kung }\end{array}$ \\
\hline Wettkampfzeit & $3 \times 1$ min & $3 \times 2$ min & $3 \times 2$ min & $3 \times 2$ min & $3 \times 2$ min \\
\hline $\begin{array}{l}\text { Pause zwischen } \\
\text { den Kämpfen }\end{array}$ & 5 Tage & 5 Tage & 5 Tage & 4 Tage & 4 Tage \\
\hline Papiergewicht & $28 \mathrm{~kg}$ & $28 \mathrm{~kg}$ & & & \\
\hline Papiergewicht & $30 \mathrm{~kg}$ & $30 \mathrm{~kg}$ & & & \\
\hline Papiergewicht & $32 \mathrm{~kg}$ & $32 \mathrm{~kg}$ & & & \\
\hline Papiergewicht & $34 \mathrm{~kg}$ & $34 \mathrm{~kg}$ & & & \\
\hline Papiergewicht & $36 \mathrm{~kg}$ & $36 \mathrm{~kg}$ & & & $38 \mathrm{~kg}$ \\
\hline Papiergewicht & $38 \mathrm{~kg}$ & $38 \mathrm{~kg}$ & $38 \mathrm{~kg}$ & $38 \mathrm{~kg}$ \\
\hline Papiergewicht & $40 \mathrm{~kg}$ & $40 \mathrm{~kg}$ & $40 \mathrm{~kg}$ & $44 \mathrm{~kg}$ & $44 \mathrm{~kg}$ \\
\hline Papiergewicht & $42 \mathrm{~kg}$ & $42 \mathrm{~kg}$ & $42 \mathrm{~kg}$ & $46 \mathrm{~kg}$ & $46 \mathrm{~kg}$ \\
\hline $\begin{array}{l}\text { Halbfliegenge- } \\
\text { wicht }\end{array}$ & $45 \mathrm{~kg}$ & $45 \mathrm{~kg}$ & $45 \mathrm{~kg}$ & $48 \mathrm{~kg}$ & $48 \mathrm{~kg}$ \\
\hline Fliegengewicht & $48 \mathrm{~kg}$ & $48 \mathrm{~kg}$ & $48 \mathrm{~kg}$ & $50 \mathrm{~kg}$ & $50 \mathrm{~kg}$ \\
\hline Bantamgewicht & $51 \mathrm{~kg}$ & $51 \mathrm{~kg}$ & $51 \mathrm{~kg}$ & $52 \mathrm{~kg}$ & $52 \mathrm{~kg}$ \\
\hline Federgewicht & $54 \mathrm{~kg}$ & $54 \mathrm{~kg}$ & $54 \mathrm{~kg}$ & $54 \mathrm{~kg}$ & $54 \mathrm{~kg}$ \\
\hline Leichtgewicht & $57 \mathrm{~kg}$ & $57 \mathrm{~kg}$ & $57 \mathrm{~kg}$ & $57 \mathrm{~kg}$ & $57 \mathrm{~kg}$ \\
\hline $\begin{array}{l}\text { Halbwelterge- } \\
\text { wicht }\end{array}$ & $60 \mathrm{~kg}$ & $60 \mathrm{~kg}$ & $60 \mathrm{~kg}$ & $60 \mathrm{~kg}$ & $60 \mathrm{~kg}$ \\
\hline Weltergwicht & $63 \mathrm{~kg}$ & $63 \mathrm{~kg}$ & $63 \mathrm{~kg}$ & $63 \mathrm{~kg}$ & $63 \mathrm{~kg}$ \\
\hline $\begin{array}{l}\text { Halbmittelge- } \\
\text { wicht }\end{array}$ & $66 \mathrm{~kg}$ & $66 \mathrm{~kg}$ & $66 \mathrm{~kg}$ & $66 \mathrm{~kg}$ & $66 \mathrm{~kg}$ \\
\hline Mittelgewicht & $69 \mathrm{~kg}$ & $69 \mathrm{~kg}$ & $69 \mathrm{~kg}$ & $70 \mathrm{~kg}$ & $70 \mathrm{~kg}$ \\
\hline $\begin{array}{l}\text { Halbschwerge- } \\
\text { wicht }\end{array}$ & $73 \mathrm{~kg}$ & $73 \mathrm{~kg}$ & $73 \mathrm{~kg}$ & $75 \mathrm{~kg}$ & $75 \mathrm{~kg}$ \\
\hline Schwergewicht & $77 \mathrm{~kg}$ & $77 \mathrm{~kg}$ & $77 \mathrm{~kg}$ & $80 \mathrm{~kg}$ & $80 \mathrm{~kg}$ \\
\hline $\begin{array}{l}\text { Superschwer- } \\
\text { gewicht }\end{array}$ & über. $77 \mathrm{~kg}$ & über. $77 \mathrm{~kg}$ & über. $77 \mathrm{~kg}$ & $86 \mathrm{~kg}$ & $86 \mathrm{~kg}$ \\
\hline $\begin{array}{l}\text { Superschwer- } \\
\text { plusgewicht }\end{array}$ & & & & über. 86 kg & $\begin{array}{l}\text { über. } 86 \\
\mathrm{~kg}\end{array}$ \\
\hline
\end{tabular}

http://www.kontaktrunde.de/boxen/gewichtsklassen.html10.10. 2003, 16:09 Uhr. 
12. Gewichtsklassen der Profiboxer

\begin{tabular}{|c|c|}
\hline Profis & Gewichtklassen \\
\hline Minifliegengewicht & $47,63 \mathrm{~kg}$ \\
\hline Juniorfliegengewicht & $48,99 \mathrm{~kg}$ \\
\hline Fliegengewicht & $50,80 \mathrm{~kg}$ \\
\hline Juniorbantamgewicht & $52,15 \mathrm{~kg}$ \\
\hline Bantamgewicht & $53,52 \mathrm{~kg}$ \\
\hline Juniorfedergewicht & $55,34 \mathrm{~kg}$ \\
\hline Federgewicht & $57,15 \mathrm{~kg}$ \\
\hline Juniorleichtgewicht & $58,96 \mathrm{~kg}$ \\
\hline Leichtgewicht & $61,23 \mathrm{~kg}$ \\
\hline Juniorweitergewicht & $63,50 \mathrm{~kg}$ \\
\hline Weltergewicht & $66,67 \mathrm{~kg}$ \\
\hline Juniormittelgewicht & $69,85 \mathrm{~kg}$ \\
\hline Mittelgewicht & $72,57 \mathrm{~kg}$ \\
\hline Supermittelgewicht & $76,20 \mathrm{~kg}$ \\
\hline Halbschwergewicht & $79,38 \mathrm{~kg}$ \\
\hline Cruisergewicht & $86,18 \mathrm{~kg}$ \\
\hline Schwergewicht & über $86,18 \mathrm{~kg}$ \\
\hline
\end{tabular}

http://www.kontaktrunde.de/boxen/gewichtsklassen.html10.10. 2003, 16:09 Uhr. 


\section{Die Kampf- und Punktrichter}

\begin{tabular}{|c|c|c|}
\hline & Name & Land \\
\hline 1 & Ali Akber Shah Qadri & (PAK) \\
\hline 2 & Alp Bartu & (TUR) \\
\hline 3 & Anatoliy Patsatyy & (UKR) \\
\hline 4 & Anthony Lohrentz & (RSA) \\
\hline 5 & Antonio Silvestri & (ITA) \\
\hline 6 & Auguste Faatau & $(\mathrm{TAH})$ \\
\hline 7 & David EtaruAgong & (UGA) \\
\hline 8 & Derenik Gabrielyan & (ARM) \\
\hline 9 & Douglas Hurtado Moyeda & (VEN) \\
\hline 10 & Edmond Folette & (SEY) \\
\hline 11 & Gaive Nagporewalla & (IND) \\
\hline 12 & Gerardus Mevissen & (AUS) \\
\hline 13 & Hachiro Yamada & $(\mathrm{JPN})$ \\
\hline 14 & Homayoun Ardanalan & $(\mathrm{IRI})$ \\
\hline 15 & Ioan Damian & (ROM) \\
\hline 16 & Janusz Nalecki & (POL) \\
\hline 17 & Julio Marmal & (DOM) \\
\hline 18 & Lyle Akelstad & (USA) \\
\hline 19 & Mahmoud Mokretari & (ALG) \\
\hline 20 & Manuel Montoya & (CUB) \\
\hline 21 & Mohamud Cassam Ackbarally Khadaroo & (MRI) \\
\hline 22 & Paiboon Srichaisawat & $(\mathrm{THA})$ \\
\hline 23 & Peter Ajtai & $(\mathrm{HUN})$ \\
\hline 24 & Rahmathan Tillyavev & $(\mathrm{UZB})$ \\
\hline 25 & Ronald Whalley & $(\mathrm{CAN})$ \\
\hline 26 & Saad Ahmed Ghonem & $(E G Y)$ \\
\hline 27 & Serikbay Abdenaliyev & (KAZ) \\
\hline 28 & Sreten Jabucanin & (YUG) \\
\hline 29 & Stanislav Kirsanov & (RUS) \\
\hline 30 & Victor Martinez & (PUR) \\
\hline 31 & Vladimir Tenev & (BUL) \\
\hline 32 & Wayne Rose & (AUS) \\
\hline 33 & Wilfried Lausch & (GER) \\
\hline 34 & Yoo Jae Joon & (KOR) \\
\hline
\end{tabular}




\section{Lebenslauf}

\section{Persönliche Angaben}

\section{Name: AYMAN RASHAD HAFEZ HUSSEIN}

Geburtsdatum: 01.03.1968

Geburtsort: EL SHARKIA / ÄGYPTEN

Familienstand: Verheiratet, 3 Kinder

Nationalität: Ägypter

\section{Ausbildung}

1974 - 1980: Grundschule (Zagazig)

1980 - 1983: Orientierungsstufe (Zagazig)

1983 - 1987: Gymnasium (Zagazig)

1987: $\quad$ Abitur (ZAGAZIG / ÄGYPTEN)

1987 - 1991: Hauptstudium, Fakultät für Sportwissenschaften der Zagazig Universität, Note: (Exzellent)

1993 - 1997: Magisterarbeit in Sportwissenschaften (Zagazig Universität) Ägypten. Thema: Developing Defensive Methodes for Novice Boxers

2000 - 2004 : Stipendiat der ägyptischen Regierung und Doktorand mit dem Ziel der Promotion an der Abteilung Gesellschaft und Training, Institut für Sportwissenschaften der Universität Göttingen, Deutschland.

\section{Erfahrung}

1980 - 1992: Boxer in der Bantamgewichtsklasse und Federgewichtsklasse sowie der Leichtgewichtsklasse bei dem Boxsportverein El Sharkia, Ägypten. 1985 - 1986: Mitglied bei den ägyptischen Meisterschaften im Bantamgewicht.

\section{Berufspraxis}

1995 - 1997: Assistent an der Fakultät für Sportwissenschaften der Universität Menofia, Ägypten.

1997 - 1999: Oberassistent an der Fakultät für Sportwissenschaften der Universität Menofia, Ägypten.

1995 - 1999: Trainer für Box- Meisterschaften in Ägypten an der Universität Menofia, Ägypten. 


\section{Erklärung}

Ich versichere, dass ich die eingereichte Dissertation (Die Finten im Boxen. Eine Untersuchung am Beispiel der Olympischen Spiele in Sydney 2000) selbständig und ohne unerlaubte Hilfsmittel verfasst habe. Anderer als die von mir angegebenen Hilfsmittel und Schriften habe ich mich nicht bedient, alle wörtlichen oder sinngemäßen Schriften habe ich kenntlich gemacht.

AYMAN RASHAD HAFEZ HUSSEIN 